Forschungszentrum Jülich

Institut für Festkörperforschung

\title{
MOCVD of High-K Ceramic Thin Films for the Gbit DRAM Technology
}

Fotios Fitsilis 



\section{MOCVD of High-K Ceramic Thin Films for the Gbit DRAM Technology}

Fotios Fitsilis 
Berichte des Forschungszentrums Jülich ; 4001 ISSN 0944-2952

Institut für Festkörperforschung Jül-4001

D 82 (Diss., Aachen, RWTH, 2002)

Zu beziehen durch: Forschungszentrum Jülich $\mathrm{GmbH} \cdot$ Zentralbibliothek D-52425 Jülich - Bundesrepublik Deutschland

o 02461/61-5220 -Telefax: 02461/61-6103 -e-mail: zb-publikation@fz-juelich.de 


\section{Abstract}

The next generation of DRAM memories demands the miniaturization of the storage capacitor. The road to smaller capacitors still able to maintain a sufficient amount of charge in terms of an error free logic state recognition leads to high-k materials. $(\mathrm{Ba}, \mathrm{Sr}) \mathrm{TiO}_{3}$ is the most promising of these new materials, since it offers a high relative permittivity combined with low leakage. Deposition of BST via MOCVD is considered to be the method of choice for thin films in view of DRAM application, in order to achieve homogenous growth and sufficient step coverage in high aspect ratio trenches. This thesis is concerned with the MOCVD growth of BST thin films using a prototype tool for oxide deposition and the systematical understanding of the film properties as a function of their composition and of the growth parameters.

The scope of this thesis is twofold. From the engineering point of view an existing MOCVD tool, the AIXTRON Planetary Reactor ${ }^{\circledR} 2600 \mathrm{G} 3$ that has been developed for the growth of III-V semiconductors, is optimized for the deposition of ceramic oxides. Both reactor and deposition processes are modified to achieve an optimal temperature and process homogeneity. Many changes on the vital components of the system like the liquid delivery system (LDS-300B) from ATMI, the precursor and the gas transfer lines are performed. Design of experiment methods (DOE) are applied early in many cases to narrow the process window and reduce the multidimensional parameter space to a manageable minimum and allow precise statements about the behavior of the reactor.

The scientific part of this thesis considers the systematic investigation of the properties of the $(\mathrm{Ba}, \mathrm{Sr}) \mathrm{TiO}_{3}$ material system in form of thin films ranging from few $\mathrm{nm}$ up to $120 \mathrm{~nm}$. Many of the structural and especially the electrical properties interesting for application are focused and a variety of analyzing techniques are applied. Within the scope of this work, an advanced $\mathrm{XRF}$ analytic procedure is developed, in order to achieve a precision $\leq 1 \%$ in thickness and stoichiometry measurements. The investigations cover all important material aspects of BST and especially its integration in the existing CMOS process. Finally, a central point in the discussion is the interrelation between the microstructure of the films and the obtained electrical properties. 


\section{Contents}

1 Introduction to the integration of ferroelectric materials................................................5

1.1 Dynamic Random Access Memory (DRAM) ....................................................5

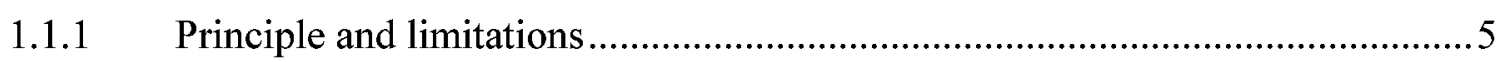

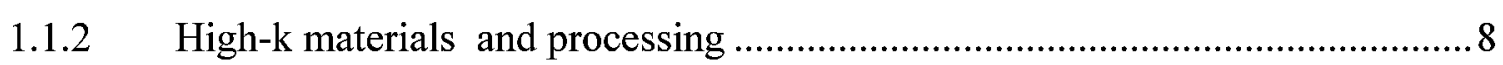

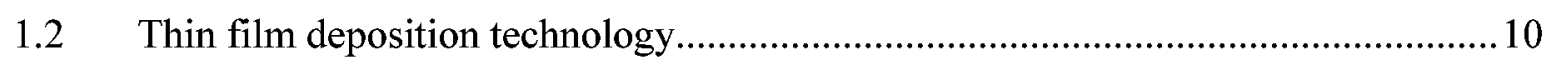

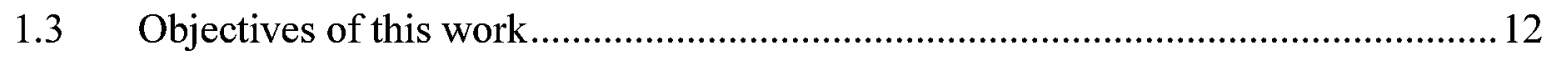

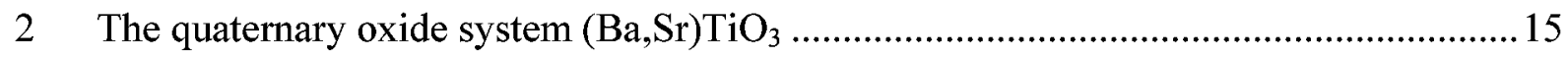

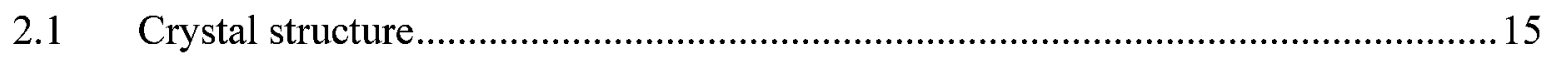

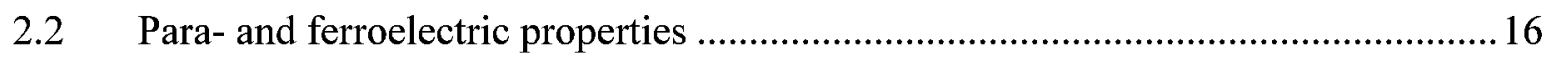

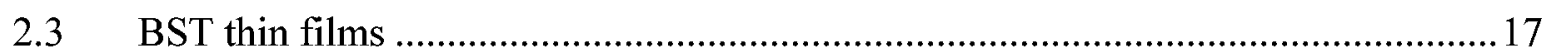

3 Fundamentals of the MOCVD for electroceramic thin films .......................................21

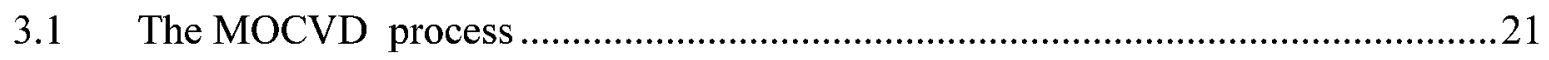

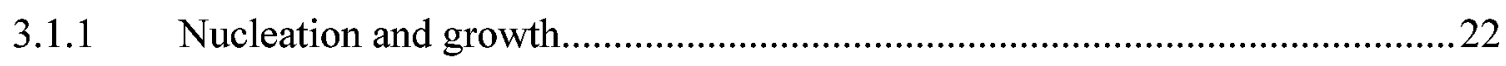

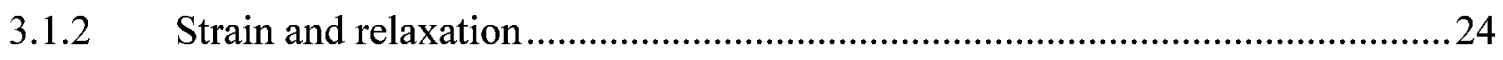

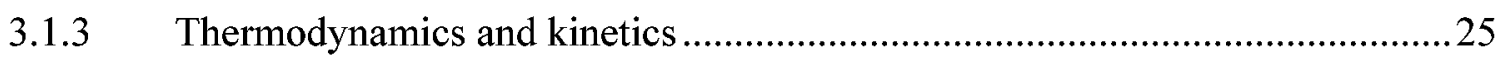

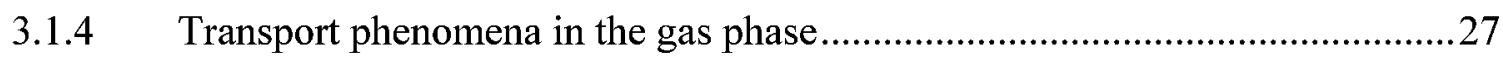

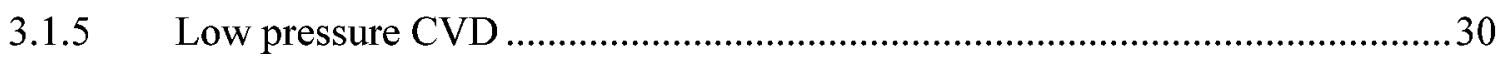

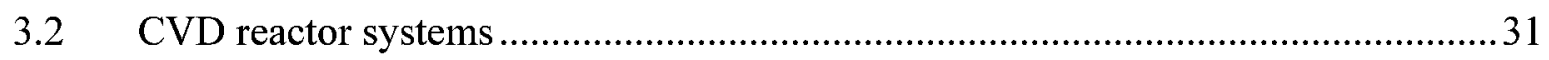

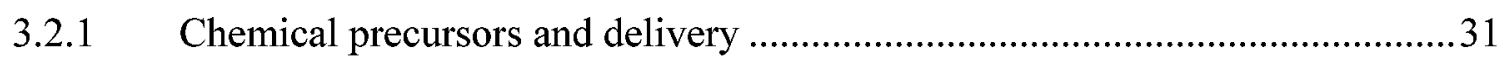

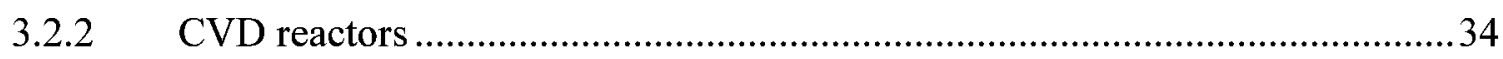

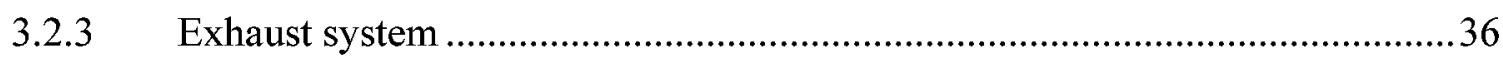

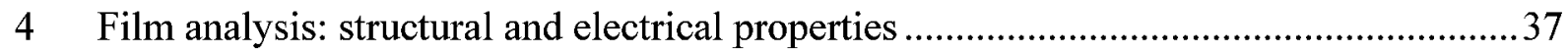

4.1 Film stoichiometry and areal mass density by X-ray fluorescence (XRF).............38

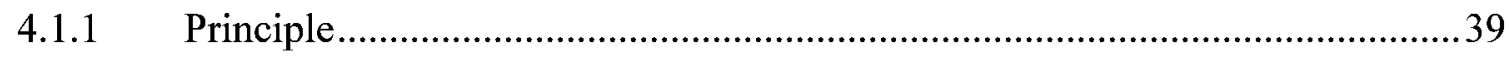

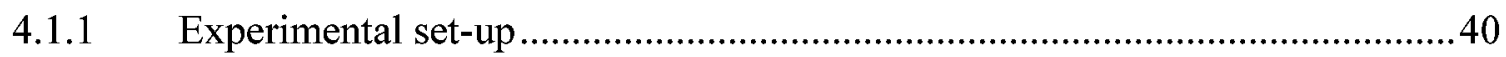

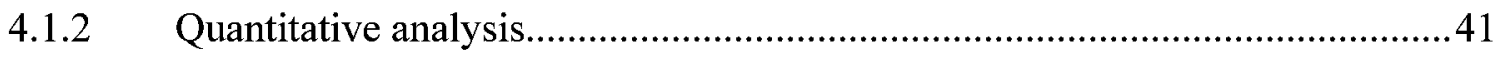

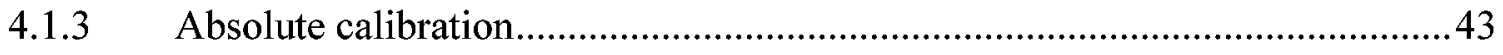

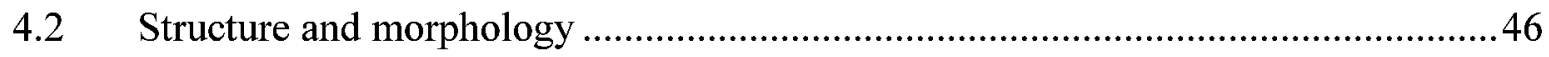

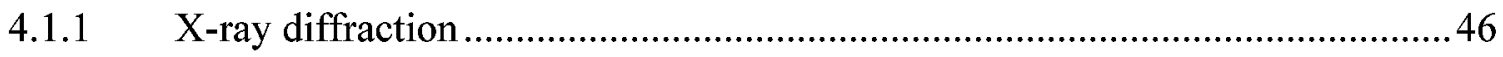

4.1.2 Depth profiling by SIMS and SNMS .........................................................

4.2.1 Fourier Transform InfraRed (FTIR) Absorption spectroscopy .......................49

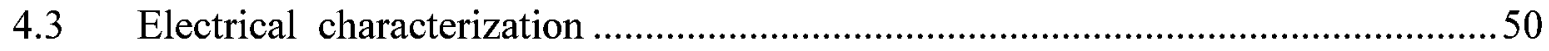

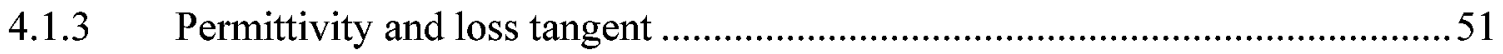

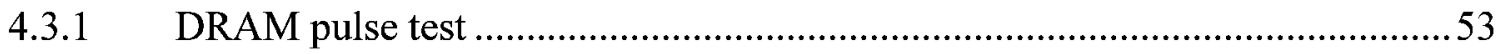




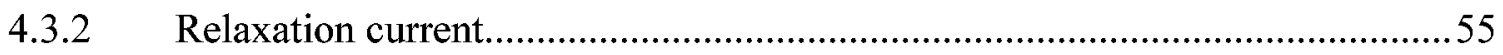

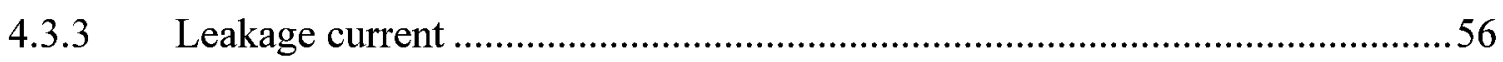

5 The AIX-2600G3 MOCVD tool and its modification for oxides .................................63

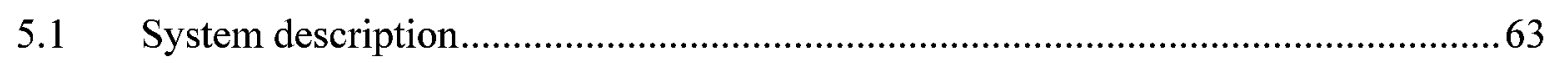

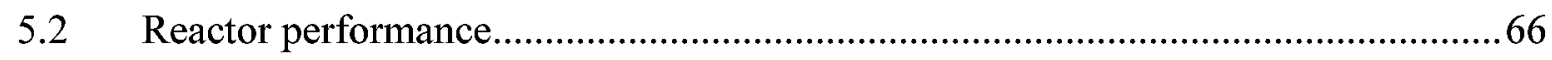

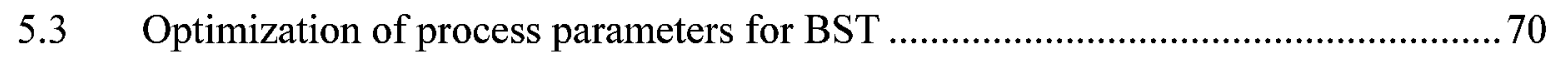

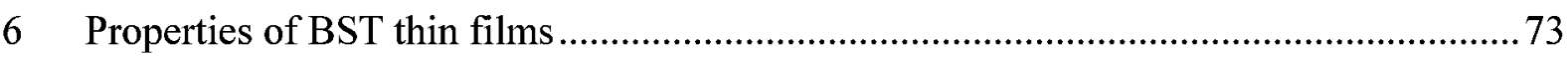

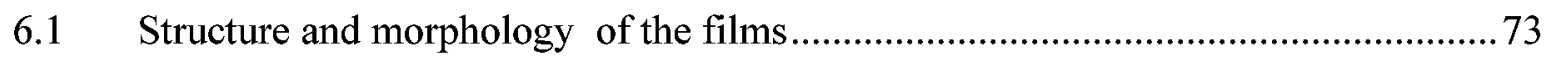

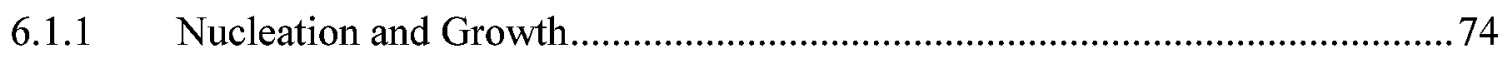

6.1.2 Dependence of the microstructure on the deposition temperature .................82

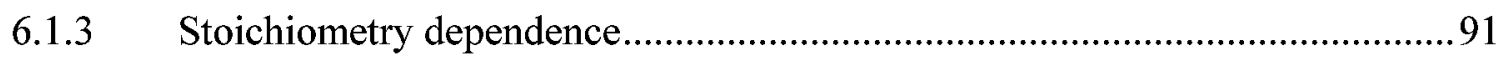

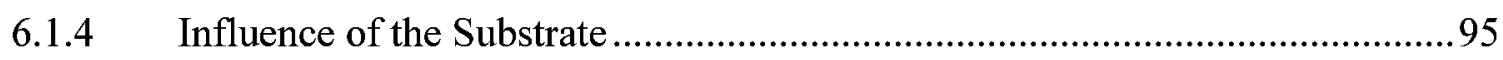

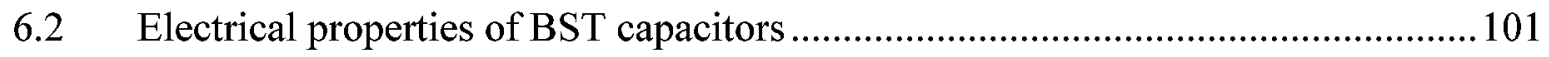

6.2.1 Properties of the electrical permittivity ........................................................... 101

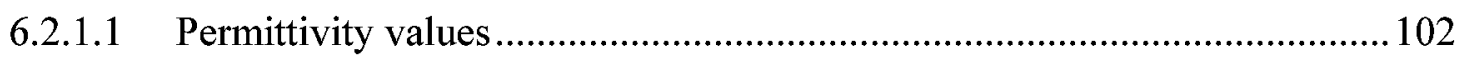

6.2.1.2 C-V characteristic and tunability ............................................................ 104

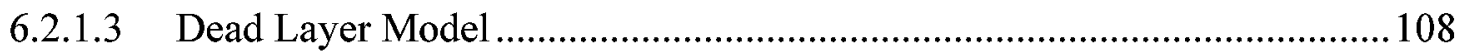

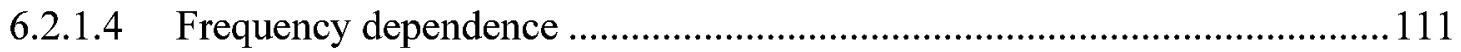

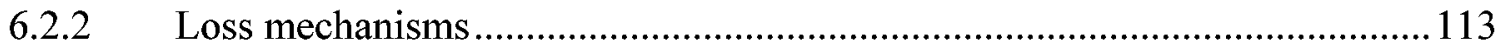

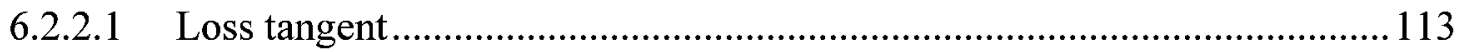

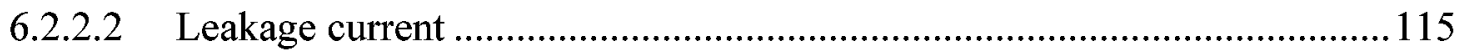

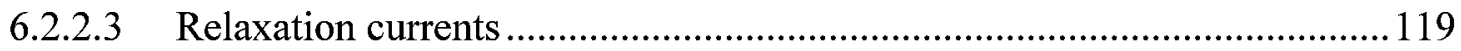

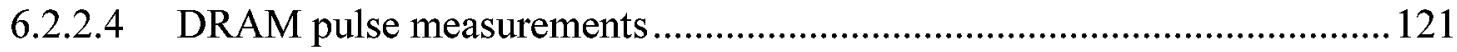

6.3 Interrelation between film microstructure and electrical properties..................... 123

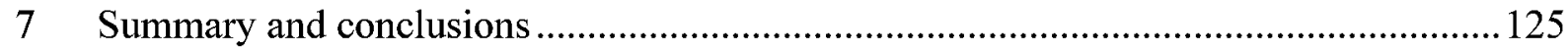

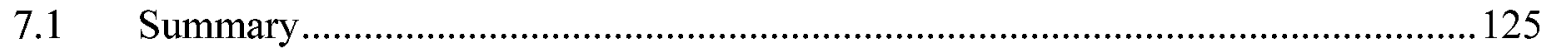

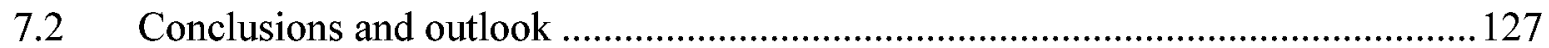

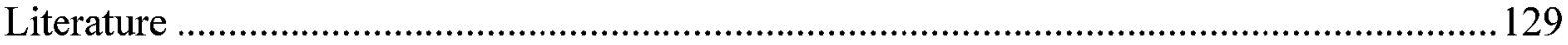

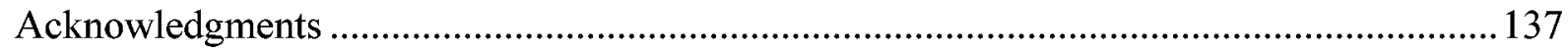




\section{Introduction to the integration of ferroelectric materials}

Electroceramic materials (ECM) have a great potential for entering into a world dominated by silicon along with a few other elements, like $\mathrm{Al}, \mathrm{B}, \mathrm{W}, \mathrm{N}$, etc., used in the most common manufacturing process called CMOS. Such materials feature ferroelectric, electro/magnetooptical, piezo- or pyroelectric properties, which all can be integrated into novel devices with unique functionality, that are difficult to achieve by conventional materials and processing. Multi-Gbit memory modules, high performance sensors and nano-actuators are examples of applications incorporating ceramic materials likely to come to the market in the near future.

High permittivity (high-k) materials have been investigated for many years and are thought to be the preamble of new dielectrics to be integrated into a CMOS device [1,2]. Their use as the capacitor's dielectric is a milestone in the development of future memory generations by replacing the conventional oxide/nitride/oxide (ONO) dielectric layers. Higher $\mathrm{k}$ is especially required for future DRAM modules, and the high-k material Barium-Strontium Titanate, BST, in a stoichiometry from $\mathrm{Ba}_{0.7} \mathrm{Sr}_{0.3} \mathrm{TiO}_{3}$ to $\mathrm{Ba}_{0.5} \mathrm{Sr}_{0.5} \mathrm{TiO}_{3}$ is considered to be a promising candidate to enter the DRAM manufacturing process. Produced in thin films, BST has a high permittivity, between 100-400, thus allowing the design of the cell in simple stack structure for the future Gbit DRAM generation.

A significant application of such capacitors is also found in tunable devices, decoupling elements or filters in microprocessors or in monolithic microwave integrated circuits (MMIC). By using the field dependency of the dielectric constant, filters and resonators can be designed that are trimmed by the applied voltage.

When replacing a capacitor's dielectric by a ferroelectric material it is possible to create non volatile memory, so called FeRAMs. The technology of these materials, such as StrontiumBismuth Tantalate (SBT) and Lead-Zirconate Titanate (PZT), is closely related to BST and similar deposition processes can be used. An important challenge for the introduction of these materials into the Gbit generation arises from the increasingly complicated wafer processing. Robust and sophisticated tools are required to make this technology less sensitive to processing variation. The integration aspects have to be considered to reduce thermal budget, while still allowing homogenous and conformal deposition of ECM. In addition, new problems arise from the promotion of interdiffusion of the different elements during postprocessing, which can affect the operation of the storage capacitor and the access transistor.

The application of novel materials in the DRAM technology is the main topic of this work, which starts with a short description of the DRAM principle, followed by the concerns that decelerate its introduction into the manufacturing process. The chapter ends with the description of the common deposition techniques and the considerations regarding the processing of BST.

\subsection{Dynamic Random Access Memory (DRAM)}

\subsubsection{Principle and limitations}

The DRAM cell consists of two basic elements. The access transistor, which is addressed through a word line connected to the gate, and a capacitor, where the information is stored in form of electric charge. A schematic 1-bit cell is depicted in Fig. 1.1. As soon as the word line is connected to the operation voltage, the transistor switches to the open state. The capacitor can then be accessed through the bit line and can be alternated/written with a zero-level voltage (logical ' 0 ') or the operating voltage (logical ' 1 '). Relative to the bottom electrode, which is permanently connected to a $V_{c c} / 2$ voltage, the capacitor will be charged with the half 
negative (' 0 ') or half positive (' 1 ') operation voltage, respectively. After the writing sequence the transistor is deactivated. Since the capacitor is not ideal, the voltage across the capacitor plates drops due to self discharge. This is why the information has to be periodically renewed in regular intervals also known as refresh intervals.

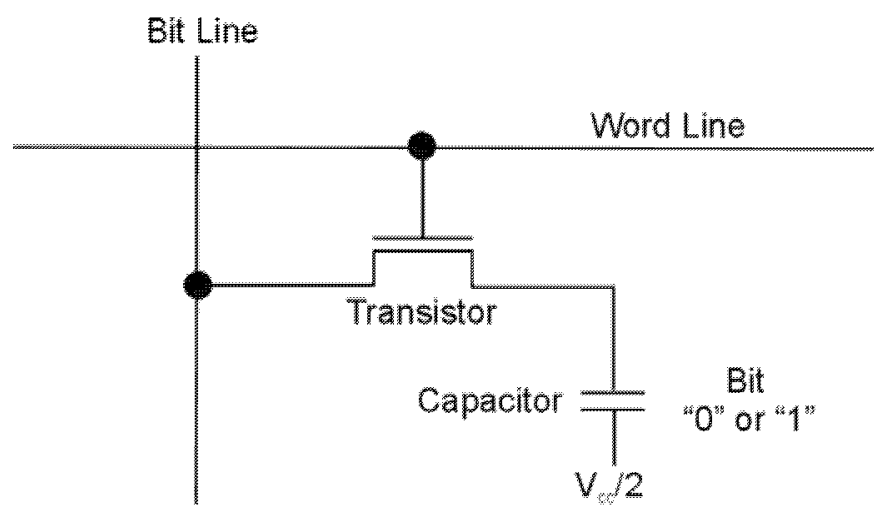

Fig. 1.1: Schematic representation of a 1T-1C DRAM cell.

The read out of the stored information is initiated by pre-charging the bit line and a reference line with $\mathrm{Vcc} / 2$. The access transistor is then activated, so that the charge in the cell capacitor flows in the parasitic bit line capacitance. This charge relocation causes a slight rise or sinking of the voltage over the bit line. The sense amplifier compares this voltage with a reference value and then interprets the difference as a logical ' 1 ' or ' 0 '. In a real memory chip the parasitic capacitance is much higher than the cell capacitance. As a result, the signal level of the voltage difference is very low $(100 \mathrm{mV}-200 \mathrm{mV})$ which makes the read out process more difficult. The read out of a DRAM is a destructive operation, so that the capacitor always has to be recharged after reading.

Since the evolution of the sense amplifiers is rather slow, the minimum charge density in the cell capacitor cannot be reduced along with the on-going miniaturization of the cell dimensions. Hence, the capacity per area on chip has to increase and all possibilities which are suggested by the capacitor formula,

$$
C=\varepsilon_{0} \varepsilon_{\mathrm{r}} \cdot A / t,
$$

where $C$ is the capacitance of a planar capacitor with the surface $A$ and thickness $t ; \varepsilon_{0}$ and $\varepsilon_{\mathrm{r}}$ represent the dielectric constant of the vacuum and the relative dielectric constant of the dielectric, have been used, i.e., reducing the thickness of the dielectric, increasing the dielectric constant and increasing the active area by 3-dimensional capacitor structures. The initially used $\mathrm{SiO}_{2}$ dielectric has been replaced by ONO coatings consisting of a $\mathrm{SiO}_{2} / \mathrm{Si}_{3} \mathrm{~N}_{4} / \mathrm{SiO}_{2}$ stack almost since the very beginning of the DRAM technology, since they offer a higher dielectric constant (approximately 7 compared to 3.9 for pure $\mathrm{SiO}_{2}$ ) plus some additional advantages regarding defect passivation and layer planarization [3]. Nevertheless, $\mathrm{SiO}_{2}$ remains the standard material for the comparison of the merits of new materials in terms of the oxide equivalent thickness $t_{o x}^{e q}$. This value describes the necessary thickness of a fictive $\mathrm{SiO}_{2}$ layer in order to achieve the same charge densities as a high-k material and is defined as follows:

$$
t_{o x}^{e q}=\left(\varepsilon_{\mathrm{SiO} 2} / \varepsilon_{\mathrm{r}}\right) \cdot t_{\mathrm{phy}}
$$


Equation 1.2 is based on the capacitor formula, Eq. 1.1, and $t_{\text {phy }}$ represents the physical thickness of the high-k dielectric, while $\varepsilon_{\mathrm{SiO} 2}$ and $\varepsilon_{\mathrm{r}}$ are the relative dielectric constants of the silicon dioxide and high-k material respectively.

The DRAM development has led to a quadrupling of the memory density on a single chip every 3 years. Additionally, the on-going miniaturization has reduced the cell size by a factor of 18.8 in the time between the $4 \mathrm{Mb}$ and the $256 \mathrm{Mb}$ generations due to major improvements in lithography and innovative processing [4]. As the detection limit of state of the art sense amplifiers lies at 20 to $30 \mathrm{fC} / \mathrm{cell}$, the minimum charge of the $1 \mathrm{~T}-1 \mathrm{C}$ cell capacitor was quickly reached for the planar design along with the shrinking of dimensions even after reducing the capacitor's thickness to a minimum. The following generations of DRAMs emerged the manufacturing of 3D capacitors in form of a stacked or a trenched cell. In the 64Mbit generation the stacked cell at $0.25 \mu \mathrm{m}$ minimum feature size required cylindrical capacitor with around $1 \mu \mathrm{m}$ height and a trench cell with the same design rule required $\approx 7-8 \mu \mathrm{m}$ depth of trench, respectively [5]. Further shrinking of the cell capacitor is translated into more complex processing in terms of deeper trenches or more complex 3D structures, which complicate wafer processing and cause increasing manufacturing costs. Fig. 1.2a presents the Mitsubishi concept for the 256Mbit capacitor and in Fig. 1.2b one can find the path followed by Toshiba (64Mb), which uses deep trench structures with aspect ratios (height/depth) up to 1:40 [6].

The ONO dielectric has a dielectric constant around 7.0, which requires an oxide-equivalent thickness around 4.5-5.0 nm and the thickness limit of ONO dielectric is considered to be around 3.5-4 $\mathrm{nm}$ in oxide equivalent thickness. Below this point, the rapid increase of the tunneling leakage current, that reduces the capacitors charge to unacceptable levels, cannot be avoided. In addition, reliability concerns arise due to the non conformal deposition on edges in high aspect ratio structures [3]. Despite this, manufacturers are still trying to push the lithographic and etching technology to its limits as demonstrated by Infineon and Mitsubishi. Nevertheless, all parties agree that the ONO trench or stack cell seems no longer appropriate for the multi-gigabit density DRAM's and the SIA roadmap considers the introduction of new high-k materials [7].
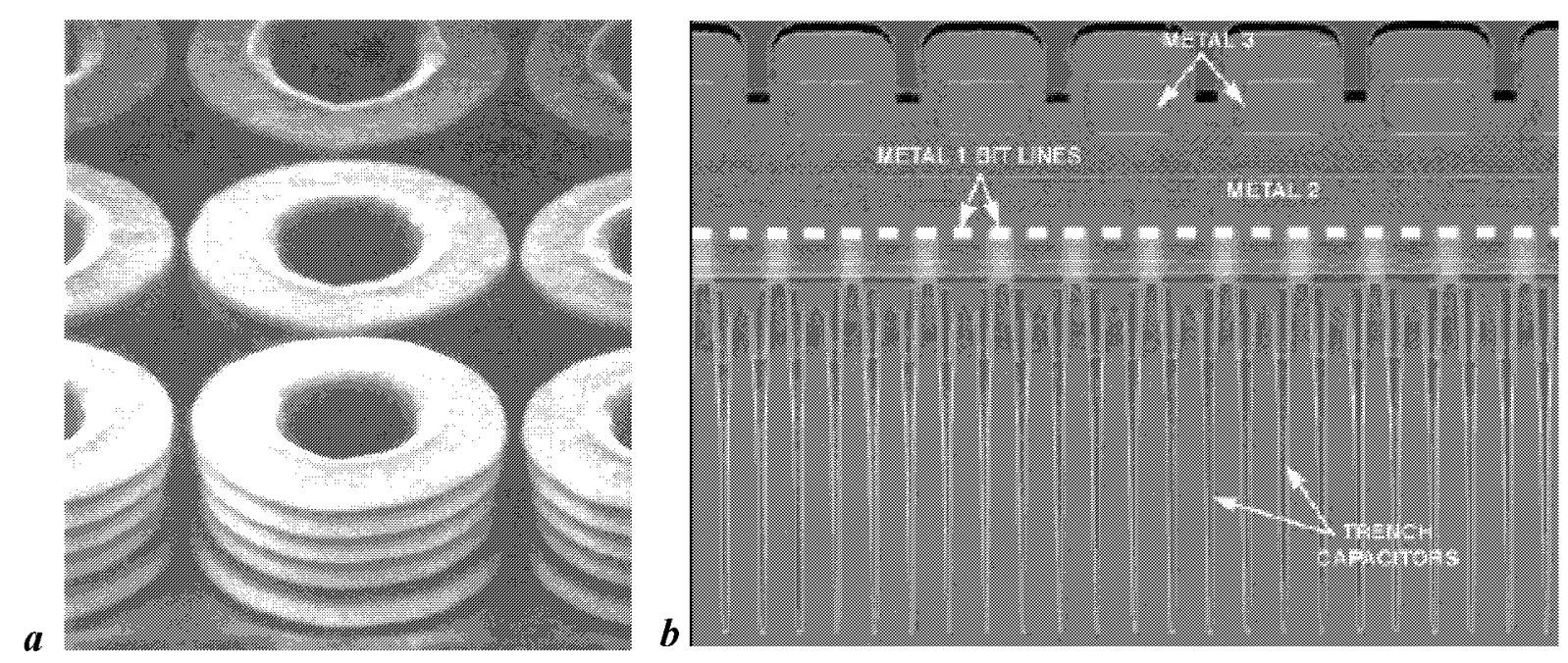

Fig. 1.2: a) Mitsubishi 256Mbit stacked-disc capacitor b) Toshiba 64Mb deep trench capacitors. 


\subsubsection{High-k materials and processing}

In terms of DRAM technology high-k materials have a dielectric constant larger than ONO and actually discussed materials range from $\mathrm{Ta}_{2} \mathrm{O}_{5}$ to BST. Table 1.1 summarizes some data from a recent near term SIA roadmap for stacked capacitors. The values proposed in the roadmap are calculated based on pedestal MIM capacitors. For the sake of simplicity we assume a constant value of $25 \mathrm{fF} /$ cell for the charge necessary for a reliable read-out. It is obvious that multi Gbit DRAMs can no longer be manufactured by conventional technology, since the theoretical oxide equivalent thickness for these devices would be smaller that the lattice constant of the oxide.

\begin{tabular}{|c|c|c|c|c|c|c|c|}
\hline $\begin{array}{l}\text { Year of } 1^{\text {st }} \\
\text { product } \\
\text { shipment }\end{array}$ & DRAM $^{*}$ & $\begin{array}{l}\text { Min. feature } \\
\text { size }(\mathrm{F})[\mathrm{nm}]\end{array}$ & $\begin{array}{c}\text { Cell } \\
\text { size } \\
{\left[\mu \mathrm{m}^{2}\right]}\end{array}$ & $\begin{array}{c}\text { Total } \\
\text { capacitor } \\
\text { area }\left[\mu \mathrm{m}^{2}\right]\end{array}$ & 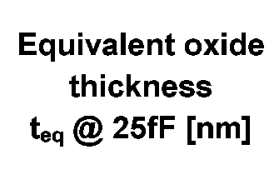 & 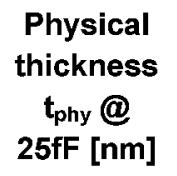 & $\begin{array}{l}\text { Dielectric } \\
\text { constant }\end{array}$ \\
\hline 2002 & $4 G$ & 130 & 0.14 & 0.88 & 1.22 & 15.6 & $50\left(\mathrm{Ta}_{2} \mathrm{O}_{5}\right)$ \\
\hline 2005 & $16 \mathrm{G}$ & 100 & 0.08 & 0.38 & 0.52 & 33.6 & 250 (BST) \\
\hline 2008 & $64 \mathrm{G}$ & 70 & 0.03 & 0.24 & 0.33 & 21.3 & 250 (BST) \\
\hline
\end{tabular}

Table 1.1: Technologv requirements for Gbit DRAMs (source: SIA [71).

*Samsung roadmap[5]

The first candidate is $\mathrm{Ta}_{2} \mathrm{O}_{5}$ and Hitachi has already presented a $256 \mathrm{Mbit}$ chip with $\mathrm{Ta}_{2} \mathrm{O}_{5}$ dielectric (Fig. 1.3). This comes much earlier than presented in the roadmap and reveals an acceleration of the proposed miniaturization trend. $\mathrm{Ta}_{2} \mathrm{O}_{5}$ is a dielectric with a permittivity around 25, however this has to be considered as an intermediate solution for a generation or two. On the other side, the roadmap is calculated with a dielectric constant of 50 for the $\mathrm{Ta}_{2} \mathrm{O}_{5}$ that is double the actual permittivity of the material. BST is suggested for the $100 \mathrm{~nm}$ technology and beyond with a permittivity value of 250 . Initially, the growth technology will be tested and optimized for mass production with a film thickness around $30 \mathrm{~nm}$. As soon as the growth mechanisms and integration process are well under control the film thickness will be decreased to approx. $20 \mathrm{~nm}$. This will be the case in the $70 \mathrm{~nm}(\mathrm{~F})$ generation. The design rule $(\mathrm{F})$ corresponds to a certain DRAM generation. This is depicted in the DRAM column based on a $8 \mathrm{~F}^{2}$ folded bit line architecture [5].

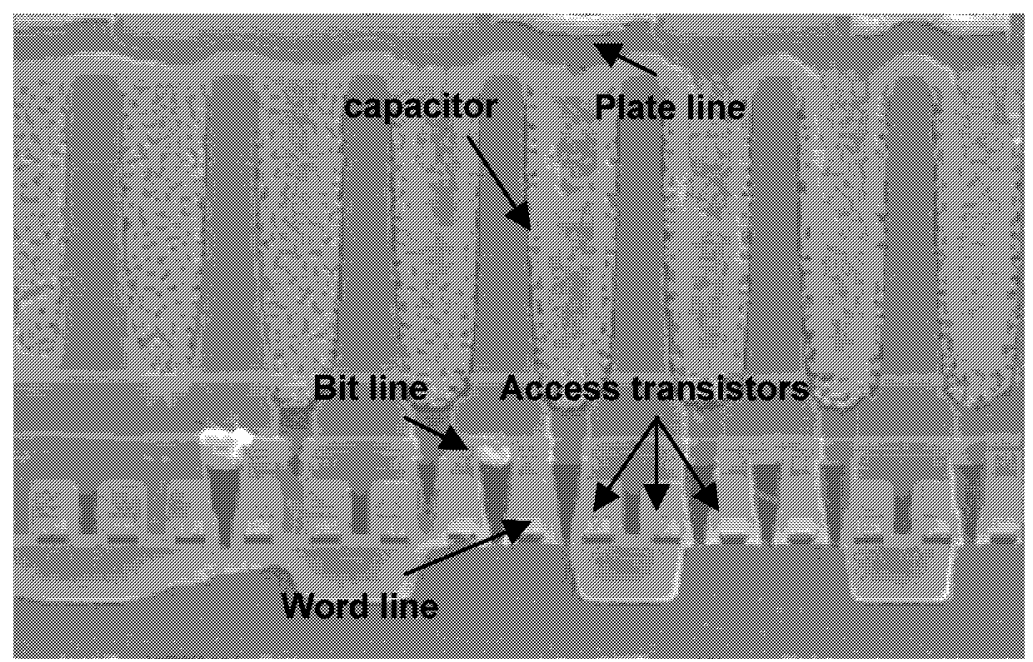

Fig. 1.3: Hitachi 256Mbit DRAM with $\mathrm{Ta}_{2} \mathrm{O}_{5}$ dielectric [8]. 
In case the DRAM concept remains unchanged in the following generations, high-k materials such as BST will be needed in order to achieve the necessary equivalent oxide thickness for the capacitor cells, even though the requirements regarding higher retention times, e.g. $1024 \mathrm{~ms}$ for the $0.07 \mu \mathrm{m}$ generation and low temperature processing $\left(<500^{\circ} \mathrm{C}\right)$, will be a great and difficult challenge for the BST dielectric. IBM has performed an advanced study in the integration of BST in form of a stacked capacitor in an attempt to examine the possibilities and prospects of using 'exotic' materials [9] The feasibility of a BST cell using a $0.2 \mu \mathrm{m}$ design rule, Pt electrodes and TaSiN barriers in a simple stacked structure was demonstrated. The IBM design approach is presented in Fig. 1.4 along with the major problems that arise from the introduction of new materials and additional processing. The electrode aspect ratio of $\sim 1.2$ that can be derived from the TEM figure and the BST film thickness of $27 \mathrm{~nm}$ meet the requirements for the $1 \mathrm{Gbit}$ generation.
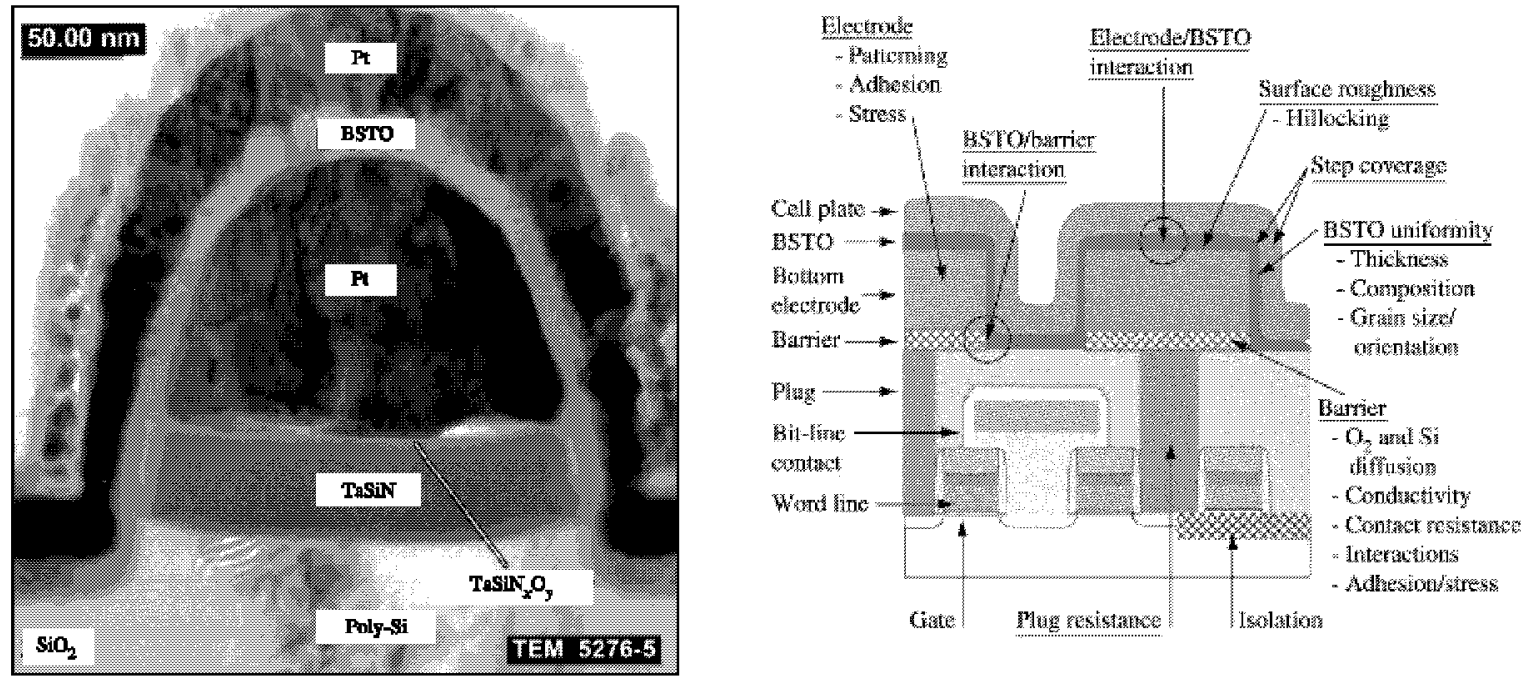

Fig. 1.4: a) Cross section TEM of a prototype Pt/BST/Pt capacitor b) Major concerns for the integration of the BST dielectric (BSTO $\equiv B S T)$.

Not only the high permittivity and the typical integration requirements, like compatibility with the standard methods, but also a large number of additional requirements have to be fulfilled in order to accept a new material in the closed family of the silicon industry. The diversity of the integration problems of high-k materials has driven many serious research efforts worldwide [9, 10-12]. In the Table 1.2 one may find the most important requirements for the thin film dielectric to be introduced in the coming Gbit generations. The second column indicates whether there is primarily a process $(P)$ or material $(M)$ requirement. Although most of the requirements may be considered as twofold, depending on both material and processing parameters, many of the problems are closely related to processing issues and this work aims to investigate some aspects of BST integration.

Finally, it should be kept in mind that BST cannot necessarily be considered as the ultimate solution of the DRAM dielectric issue and soon we will face the same cell complexity problems, unless the permittivity of the dielectric is further increased. The SIA roadmap uses rather high permittivity values for BST around 700, in order to maintain the pedestal stack structure in the $0.05 \mu \mathrm{m}$ generation and beyond, which is unachievable for the present state of 


\begin{tabular}{l|c}
\multicolumn{1}{c|}{ Requirements } & Process/Material \\
\hline $\begin{array}{l}\text { Homogenous thin film deposition (<30nm) over large substrate areas } \\
\text { Conformal deposition of 3D structures with high aspect ratios }\end{array}$ & $\mathrm{P}$ \\
$\begin{array}{l}\text { Reproducible film deposition regarding thickness and stoichiometry to } \\
\text { ensure long term process stability }\end{array}$ & $\mathrm{P}$ \\
$\begin{array}{l}\text { Compatibility to CMOS process / Low thermal budget (Growth temperature } \\
\left.\text { below } 500^{\circ} \mathrm{C}\right)\end{array}$ & $\mathrm{P}$ \\
$\begin{array}{l}\text { High throughput for high volume production } \\
\left.\text { High permittivity ( } \varepsilon_{\mathrm{r}}>100\right) \text { to guarantee simpler capacitor geometry for the } \\
\text { next } 1-2 \text { memory generations }\end{array}$ & $\mathrm{P}$ \\
$\begin{array}{l}\text { Reduced leakage current to ensure rare refresh intervals }(\approx 1 \text { sec) with less } \\
\text { than } 10 \% \text { charge loss due to self discharge during inter-refresh times }\end{array}$ & $\mathrm{M}$ \\
$\begin{array}{l}\text { Development of barrier and electrode materials compatible to current CMOS } \\
\text { processing }\end{array}$ & $\mathrm{M} / \mathrm{P}$ \\
$\begin{array}{l}\text { Development of novel etching processes for the dielectric material, } \\
\text { electrodes and barriers } \\
\text { Long term degradation stability (in excess of } 10 \text { years operation stability) }\end{array}$ & $\mathrm{M}$ \\
\hline
\end{tabular}

Table 1.2: Requirements set by the DRAM manufacturers on the technology (or process, $P$ ) and the material system $(M)$.

the art BST films and more basic research is needed in terms of interface effects that lead to a shrinking of the effective dielectric constant in thin films (see Chapter 6.4). From the above it can be concluded that the problem is focused on the charge read out, but alternative approaches are possible, like the development of resistive RAMs where the logic level is determined by resistivity measurements [13]. Ultimately, it is an economic decision, between the investment in new tools and processes for pushing the existing technology to further limits and the introduction of a completely new concept.

\subsection{Thin film deposition technology}

Electroceramic materials are in transition from the laboratory to mass production. The key to this transition will be the deposition technology for thin film production. Thin films in the ULSI technology will have a thickness of a few tens $\mathrm{nm}$ or below, and depending on the application, it will be necessary to have amorphous, oriented or epitaxial growth of these materials on different substrates. There is a number of different deposition techniques that are suitable to grow materials as thin films. The use of one specific technology strongly depends on the material system itself as well as integration parameters and targeted properties. Beside the wet chemical routes for thin film fabrication via chemical solution deposition (CSD) there are methods for film forming out of the gas phase. The latter are referred as physical (PVD) or chemical vapor deposition (CVD). All these methods have been tested and are suitable for thin film growth on planar substrates up to $200 \mathrm{~mm}$ diameter. The following table presents an overview of different deposition methods with the most characteristic features in the field of interest, the deposition of ceramic films.

The CSD method uses a solution of metal-organic compounds at room temperature to coat an arbitrary substrate mostly in a spin-on process. After a drying phase, where the film becomes 


\begin{tabular}{|c|c|c|c|c|c|}
\hline \multirow{3}{*}{$\begin{array}{l}\text { Mechanism of } \\
\text { production of } \\
\text { depositing species }\end{array}$} & \multicolumn{3}{|c|}{ Physical Vapor Deposition } & \multicolumn{2}{|c|}{ Chemical deposition } \\
\hline & Evap./MBE & Sputtering & PLD & CVD / MOCVD & CSD \\
\hline & Thermal energy & $\begin{array}{l}\text { Momentum } \\
\text { transfer }\end{array}$ & $\begin{array}{l}\text { Thermal } \\
\text { energy }\end{array}$ & $\begin{array}{l}\text { Chemical } \\
\text { reaction }\end{array}$ & $\begin{array}{l}\text { Deposition } \\
\text { from } \\
\text { solution }\end{array}$ \\
\hline Deposition rate & $\begin{array}{l}\text { High, up to } \\
750 \AA / \mathrm{min}\end{array}$ & $\begin{array}{l}\text { Low, except } \\
\text { for pure metal }\end{array}$ & Moderate & $\begin{array}{l}\text { Moderate, } \\
\text { up to } 25 \AA / \mathrm{min}\end{array}$ & Multi-cycle \\
\hline Deposition species & Atoms and ions & $\begin{array}{l}\text { Atoms and } \\
\text { ions }\end{array}$ & $\begin{array}{l}\text { Atoms, ions } \\
\text { and clusters }\end{array}$ & Atoms & $\begin{array}{l}\text { Solute } \\
\text { complexes }\end{array}$ \\
\hline $\begin{array}{l}\text { Energy of deposit- } \\
\text { species }\end{array}$ & $\begin{array}{l}\text { Low } \\
0.1 \text { to } 0.5 \mathrm{eV}\end{array}$ & $\begin{array}{l}\text { Can be high } \\
1-100 \mathrm{eV}\end{array}$ & Low to high & $\begin{array}{l}\text { Can be high } \\
\text { with Plasma-aid }\end{array}$ & low \\
\hline $\begin{array}{l}\text { Throwing power } \\
\text { a) Complex shaped } \\
\text { Object } \\
\text { b) Into blind hole }\end{array}$ & $\begin{array}{l}\text { Poor, line of } \\
\text { Sight } \\
\text { Poor }\end{array}$ & $\begin{array}{l}\text { Nonuniform } \\
\text { thickness } \\
\text { Poor }\end{array}$ & $\begin{array}{l}\text { Poor } \\
\text { Poor }\end{array}$ & $\begin{array}{l}\text { Good } \\
\text { Limited }\end{array}$ & Poor \\
\hline
\end{tabular}

Table 1.3: Characteristics of deposition methods [14].

a gel-like form, a rapid thermal annealing step between $500-700^{\circ} \mathrm{C}$ is needed, where the perovskite phase is formed. This step can be repeated to reach the desirable film thickness, but has a considerable effect on the thermal budget. This can be time consuming and consequently not suitable for mass production. CSD is often used for prototyping and laboratory scale thin film growth. Since the substrate is initially covered by a liquid, it is very difficult, if not impossible, to achieve conformal deposition of 3D structures due to the capillary effect. However, sophisticated routes and processes have evolved for low scale integration (LSI) [15]. Although CSD is dominantly used for the deposition of thicker films, e.g. for ferroelectric and piezoelectric applications, very thin films have been deposited recently and the thinnest films so far produced by CSD have a thickness of around $15 \mathrm{~nm}$ and there are several efforts to produce even thinner films [16].

PVD includes molecular beam epitaxy (MBE), pulsed laser deposition (PLD) and sputtering. All these methods have in common that the target material is deposited on the substrate in the same phase and composition. The target is being hit by a focused laser beam (PLD) or fast ions (sputtering) which leads to a heating of the target and subsequent emission of atoms in the direction of the wafer. By adding oxygen, the ceramic film may grow in situ without post-growing annealing. It is generally difficult to achieve conformal deposition of threedimensional structures. This is only possible if the entire geometry of the structure is in the line of sight of the emitted atoms, since they follow ballistic routes after their emission.

Metal Organic Chemical Vapor Deposition (MOCVD) appears to be the most suitable one to grow ceramic thin films on a mass production scale. CVD techniques have been widely used for the deposition of thin films in many fields of modern technology and are standard processes in the CMOS technology for the deposition of insulators and interlayer dielectrics like $\mathrm{SiO}_{2}$ and $\mathrm{SiN}_{\mathrm{x}}$. In case of epitaxial growth of III-V semiconductors (GaAs, InP) using hydrogen containing precursors CVD is often referred as VPE, vapor phase epitaxy. CVD offers good conformal deposition over the complex 3D device topographies common to ULSI-scale compared to physical deposition methods such as sputtering or evaporation methods. A huge advantage of CVD is the high deposition rate and the amenability to large 
wafer-size scaling. CVD tools can currently handle state of the art 8 inch wafers $(200 \mathrm{~mm})$ and the development goes towards 12 inch $(300 \mathrm{~mm})$. This is why CVD is considered as the prime deposition technique in this field.

In CVD, film growth occurs through the vapor phase transport of chemical components, which are called precursors, in the reactor, where they react on a heated substrate. Due to the relatively low process temperatures, the thermal budget can be decreased significantly. The film forming reactions are typically activated by thermal energy from a heated substrate, but other energy sources can be used such as RF or microwave power to reduce the thermal energy contribution. For the processing of many metals, special precursors in the form of organometallic compounds had to be developed to obtain a sufficient volatility. The interest in high- $\mathrm{T}_{\mathfrak{c}}$ superconductors has fueled the development of deposition techniques for perovskite type metal oxides. Especially, the perovskite oxides of interest in electroceramic films, i.e. high epsilon materials like ( $\mathrm{Ba}, \mathrm{Sr}) \mathrm{TiO}_{3}(\mathrm{BST})$ for advanced DRAM concepts as well as ferroelectric materials like $\mathrm{Pb}(\mathrm{Zr}, \mathrm{Ti}) \mathrm{O}_{3}$ (PZT) and $\mathrm{SrBi}_{2} \mathrm{Ta}_{2} \mathrm{O}_{9}$ (SBT) for ferroelectric memories have largely benefited from this development $[17,18]$.

Nevertheless, the lack of reliable production tools on the market and the control of the numerous process parameters have prevented the introduction of high-k dielectrics into the semiconductor Fab. The parameters of the precursor delivery system are especially crucial for a controlled film growth. In a preceding thesis Schäfer has evaluated different systems on a laboratory scale including an ultrasonic vaporizer to generate precursor aerosols and the most common method flash evaporation on a hot surface [19]. For the present investigation on a production scale tool we used the most advanced commercial system, the liquid delivery system 300B from ATMI, which includes a computer controlled mixing of liquid precursors from different sources and a flash evaporator. This system was implemented in a AIX$2600 \mathrm{G} 3$ planetary reactor with a five times 6-inch wafer capacity. This tool allows for the first time the evaluation of a batch processing for a reliable large scale production of BST thin films.

\subsection{Objectives of this work}

The research described in this work centers around a prototype for a production capacity MOCVD tool for deposition of ECM materials, primarily BST. The nature of a prototype tool is that it has to be further developed to meet the requirements set by the semiconductor industry in terms of high quality thin films and reproducibility of the results. Downtime and maintenance intervals have to be reduced to a minimum. In addition to the tool's development, stable deposition processes have to be developed within a multi-dimensional parameter space. The main objective of this work is the deposition of BST thin films of high quality in a thickness range from ultra thin films $<10 \mathrm{~nm}$ up to $120 \mathrm{~nm}$ and the structural and electrical characterization of these films. The characterization of the films was performed in the parameter region interesting for application, e.g. the operation temperature was varied between room temperature $\left(\approx 25^{\circ} \mathrm{C}\right)$ and $150^{\circ} \mathrm{C}$.

The present work may be subdivided into two parts. First, the development of tools for film deposition and characterization is presented. In the second part we discuss the properties of thin BST films. Hence, the most important aspects of this thesis are summarized in the following: 


\section{A. Technical development (Chapters 4 and 5)}

- Probe stations for the characterization of thin films:

(i) Process development on the X-ray fluorescence (XRF) analysis technique for the examination of ECM thin films. The achievement of high precision composition control required the development of special measurement procedures for thin films. A detailed description will be given in Chapter 4.1.1.

(ii) Electrical characterization (Chapter 4.2): Focus on properties useful for DRAM operation like permittivity, C-V characteristic, dissipation factor, relaxation phenomena, leakage currents and simulation of DRAM operation.

- MOCVD tool:

(i) Start-up operation, development and optimization of the MOVCD tool. Testing of new components and improvement of existing features like temperature homogeneity of the reactor.

(ii) Development and optimization of processes for thin film deposition of the $\mathrm{Ba}_{0.7} \mathrm{Sr}_{0.3} \mathrm{TiO}_{3}$ oxide material system as a prototype high-k dielectric.

\section{B. Properties of BST thin films (Chapter 6)}

- Deposition of different BST films within a wide parameter space: Influence of process parameters and control of the film microstructure.

- Characterization and analysis of the films:

(i) Chemistry by XRF

(ii) Structure using X-Ray Diffraction (XRD), FTIR and SIMS/SNMS

(iii) Morphology and microstructure of the surface by use of a wide spectrum of available surface analytical methods such as HRTEM, SEM and AFM

(iv) Electrical characterization

- Growth studies of ultra thin BST films: from seed growth to a continuous film.

- Correlation of the electrical properties to the microstructure of BST thin films.

This study mainly aims to demonstrate the feasibility of an advanced MOCVD process to grow high quality BST thin films. The final goal of this investigation is the systematic understanding of the correlation between thin film structure and properties. 


\section{The quaternary oxide system $(\mathrm{Ba}, \mathrm{Sr}) \mathrm{TiO}_{3}$}

The scope of this thesis is focused on the characterization of $(\mathrm{Ba}, \mathrm{Sr}) \mathrm{TiO}_{3}$ thin films. The structure and the properties of $\mathrm{SrTiO}_{3}$ and $\mathrm{BaTiO}_{3}$ and the mixed oxide system $(\mathrm{Ba}, \mathrm{Sr}) \mathrm{TiO}_{3}$ (BST or BSTO) was intensively investigated for more than 50 year by many international groups and the bulk properties of this material are summarized in actual textbooks [20]. However, a thin film does not always display the same behavior as the bulk and needs special consideration. This chapter gathers the BST properties of interest for the present technologically oriented work.

\subsection{Crystal structure}

The metal oxide system $\left(\mathrm{Ba}^{2+} \mathrm{Sr}^{2+}\right) \mathrm{Ti}^{4+} \mathrm{O}_{3}{ }^{2-}$ is a mixed crystal system with the perovskite structure of the general type $\mathrm{A}^{2+} \mathrm{B}^{4+} \mathrm{O}_{3}{ }^{2-}$, as shown in Fig. 2.1a. The name perovskite comes from the mineral $\mathrm{CaTiO}_{3}$, but is now used to describe a whole class of materials with the same cubic structure or similar distorted crystals. The large $\mathrm{A}^{2+}$ cation in the perovskite lattice is represented by $\mathrm{Sr}$ or $\mathrm{Ba}$ cations with similar ion radii. The smaller Ti cation in the B-position is in the middle of an octahedron of $\mathrm{O}^{2-}$ anions. The chemical bonds in metal oxides are mostly ionic.
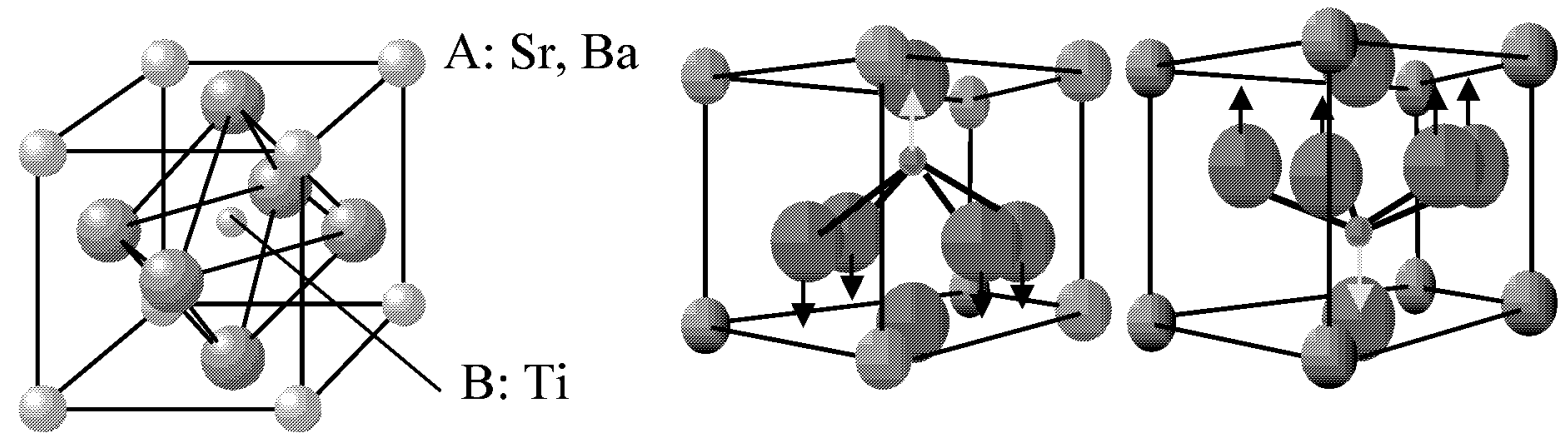

Fig. 2.1: a) Cubic perovskite cell b) Displacement of the Ti ions at the transition to the ferroelectric phase.

The distortion of the cell structure strongly depends on the ionic radii of the cations and the different phases of bulk ceramics have been investigated in detail and are summarized in Fig. 2.2. The values for the $\mathrm{Sr}, \mathrm{Ba}$ and the Ti cations are $1.16 \AA, 1.35 \AA$ and $0.65 \AA$ respectively and the radius of $\mathrm{O}^{2-}$ anion was determined to $1.39 \AA$ [21]. The figure shows that $\mathrm{SrTiO}_{3}$ has a cubic cell structure at room temperature and consequently must be paraelectric. However, $\mathrm{SrTiO}_{3}$ shows a structural phase transition to a tetragonal phase at $28 \mathrm{~K} . \mathrm{BaTiO}_{3}$ displays this phase transition at $130^{\circ} \mathrm{C}$ and is tetragonal at room temperature. This tetragonal structure is expressed in the displacement of the $\mathrm{Ti}^{4+}$ ions in the crystal lattice (Fig. 2.1). These two materials can be fully mixed into BST, so that the phase transition is adjustable over a wide temperature range. 


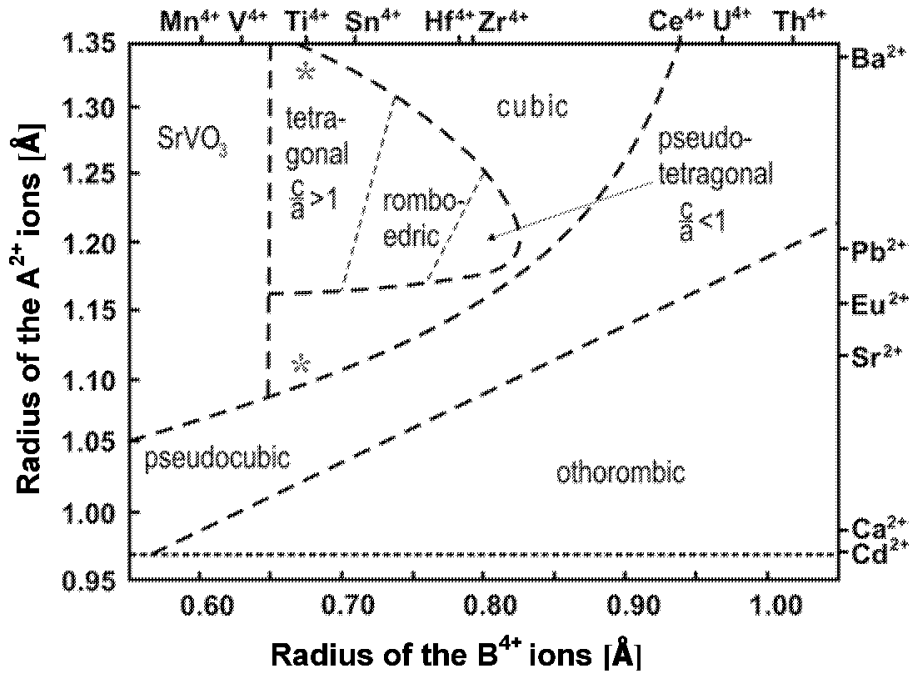

Fig. 2.2: Distorted perovskites $A^{2+} B^{4+} O_{3}^{2-}$ and their dependence on the ion radii $R_{A}, R_{B}$. at room temperature. $\mathrm{SrTiO}_{3}(\mathrm{STO})$ displays a cubic cell and $\mathrm{BaTiO}_{3}$ (BTO) a tetragonal structure. Through proper mixing of both components it is possible to adjust the desired transition temperature. The points represent the crystal structure of BTO and STO at room temperature. Taken from [22].

\subsection{Para- and ferroelectric properties}

BST is paraelectric in the cubic high temperature phase and ferroelectric properties are displayed below the phase transition temperature, $T_{0}$. Ferroelectricity is characterized through a spontaneous polarization (Fig. 2.3) of the crystalline material in the direction of the tetragonal axis. The polarization can be switched through an external field (Fig. 2.1b).

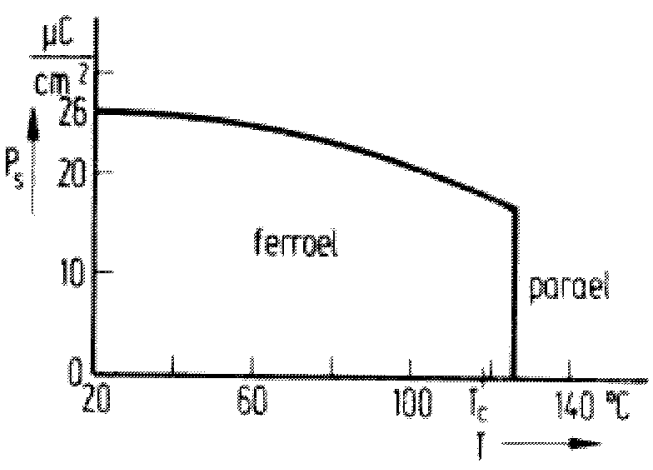

Fig. 2.3: Spontaneous polarization of a $\mathrm{BaTiO}_{3}$ single crystal as a function of temperature. The transition temperature is $\sim 130^{\circ} \mathrm{C}$; below this temperature the materials remains ferroelectric with a remanent polarization of $17 \mu \mathrm{C} / \mathrm{cm}^{2}$, which displays a slow increase with decreasing temperature up to $26 \mu \mathrm{C} / \mathrm{cm}^{2}$ at $20^{\circ} \mathrm{C}$ [23].

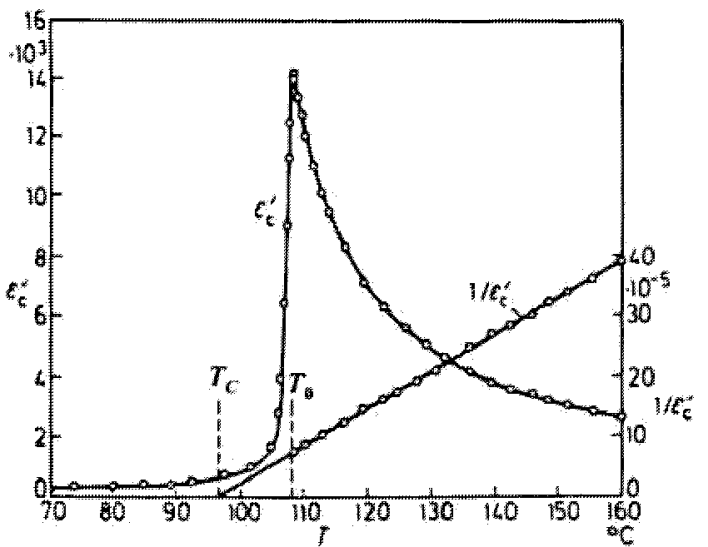

Fig. 2.4: Dielectric constant $\mathcal{E}^{\prime}$ for $B T O$ measured parallel to the $c$ axis with an alternating field of a frequency $1 \mathrm{kHz}$. An extremely pure single crystal was used. The transition $T_{0}$ depends on the purity of the sample [24].

The transition is also associated with a strong rise of the permittivity, which reaches values up to 10000 for $\mathrm{BaTiO}_{3}$ (see Fig. 2.4). Above the Curie temperature $T_{\mathrm{c}}$, the permittivity obeys the empirical Curie-Weiss law where $\mathrm{C}$ is the Curie-constant and $T_{\mathrm{c}}$ is the Curie temperature, which is generally smaller than the temperature $T_{0}\left(T_{\mathrm{c}}<T_{0}\right)$ of the phase transition from the cubic to the tetragonal lattice and vice versa, Fig. 2.5. For $\mathrm{SrTiO}_{3}$ a CurieWeiss behavior of the permittivity is observed over a wide temperature range, however, no ferroelectricity has been observed below the phase transition at $28 \mathrm{~K}$ so far. 


$$
\varepsilon_{r}^{\prime}(T) \approx \chi^{\prime}(T)=\frac{C}{T-T_{c}}
$$

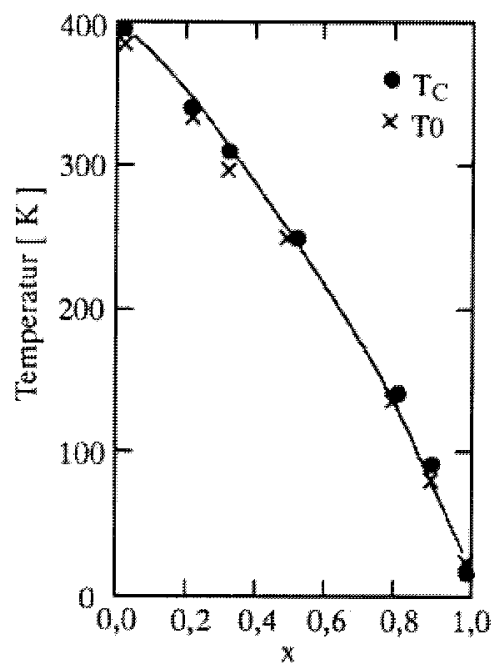

Fig. 2.5: Temperature dependence of the permittivity of BST ceramics for different compositions ranging from BTO to STO [25].
Eq. 2.1

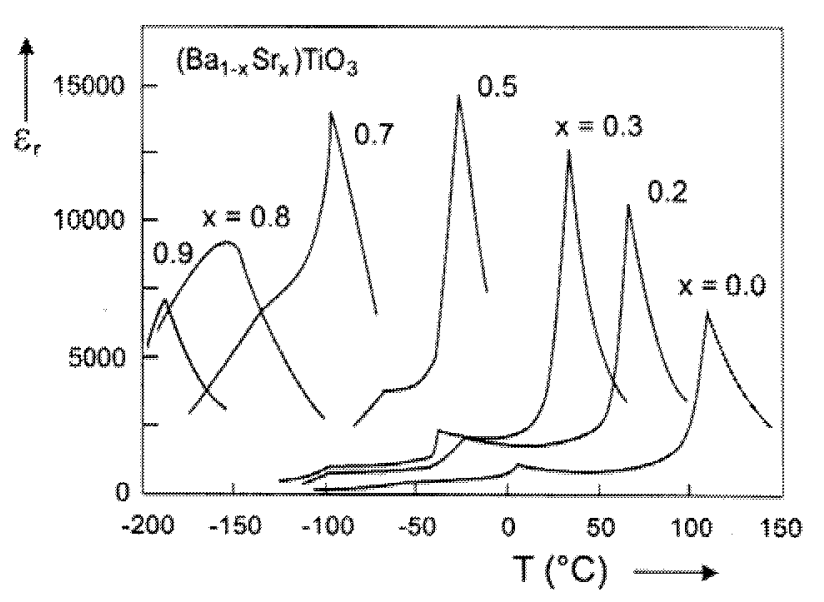

Fig. 2.6: Composition dependence of the transition $\left(T_{0}\right)$ and Curie $\left(T_{c}\right)$ temperature [26].

As illustrated in Fig.2.6, which shows the temperature dependence of the low frequency dielectric constant in $\left(\mathrm{Sr}_{\mathrm{x}} \mathrm{Ba}_{1-\mathrm{x}}\right) \mathrm{TiO}_{3}$ ceramics in the compositions from $\mathrm{x}=0, \mathrm{BaTiO}_{3}$, to $\mathrm{x}=1$, $\mathrm{SrTiO}_{3}$, the ferroelectric transition of BST can be shifted in temperature by adjusting the $\mathrm{Ba}$ to Sr ratio in the BST lattice. Through proper mixture of the two base components not only the $T_{0}$ but also the maximum of the permittivity can be set appropriately in order to be suitable for DRAM applications. For bulk ceramics this maximum is near room temperature for a $\mathrm{Ba}: \mathrm{Sr}$ ratio of $7: 3$. However, the maximum of the permittivity in bulk BST always appears as a sharp peak which would require extremely high temperature control for applications.

\section{3 $\quad$ BST thin films}

In the previous caption we summarized the properties of bulk polycrystalline BST. Thin films with thickness $<1 \mu \mathrm{m}$ display a deviation from these properties. This different behavior may be induced by grain size effects as well as by substrate properties like thermally induced stress due to different thermal expansion coefficients or interfacial space charges.

Dietz found permittivity values between 150 and 300 for STO thin films grown by CSD on Pt electrodes [27]. Thin BST films display a strong deviation of the permittivity compared to the bulk case as can be seen in Fig.2.7 a and b [28]. The permittivity values lie in the region $\varepsilon_{\mathrm{r}}$ 250-1000. The maximum of the permittivity for thin films is spread over a rather wide temperature range, which is advantageous for device operation. On the other side, the permittivity of thin films is lower by at least 1.5 orders of magnitude compared to the bulk, but still remains high compared to conventional ONO dielectrics $\left(\varepsilon_{\mathrm{r}}^{\mathrm{ONO}} \sim 6-9\right)$. Part $\mathrm{b}$, which presents a plot of $1 / \varepsilon_{\mathrm{r}}$ shows the corresponding larger deviations from the Curie-Weiss line and the suppressed phase transition. 


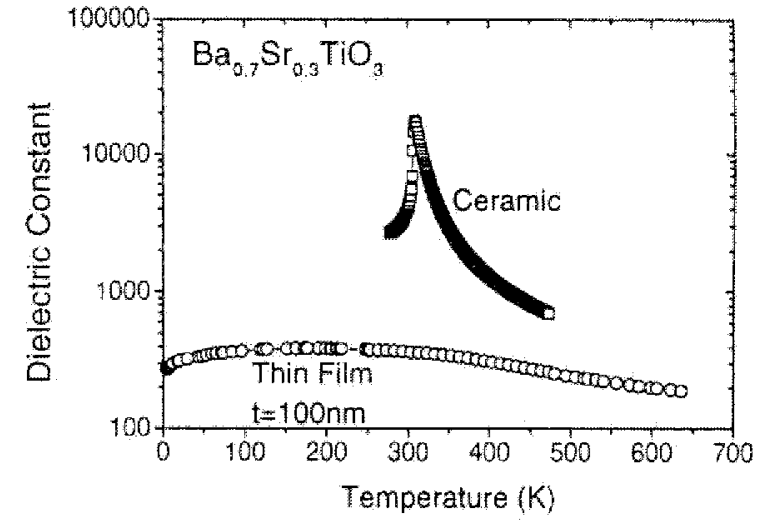

Fig. 2.7a: Temperature dependence of the Permittivity for ceramic and thin film dielectrics of the composition $\mathrm{Ba}_{0.7} \mathrm{Sr}_{0.3} \mathrm{TiO}_{3}$. Thin films display a wide distibution of the permittivity maximum, in contrast to the bulk case, where we obtain a sharp maximum at $\sim 330 K$ [28].

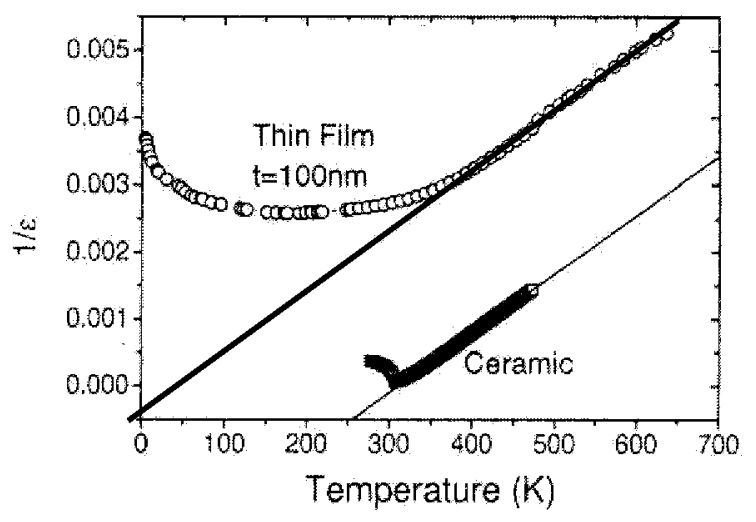

Fig. 2.7b: Temperature dependence of the inverse relative permittivity $\left(\varepsilon_{r}^{-1}\right)$ at zero field for a $100 \mathrm{~nm}$ thin BST film. The extrapolation yields a $T_{c}$ at very low temperatures [28].

Grain size effects on the permittivity of fine grain ceramics are well known. Arlt and Hoffmann compiled this dependence for bulk and thin film $\mathrm{BaTiO}_{3}$ (Fig.2.8) [15, 29]. The permittivity first increases with decreasing grain size $\left(t_{\mathrm{G}}\right)$ and reaches a maximum for a $t_{\mathrm{G}}$ $\sim 700 \mathrm{~nm}$. A further decrease of the grain size below this point leads to a decrease of the relative permittivity. This behavior can be explained by two competing effects. The increase of the permittivity is believed to be due to mechanical stress. Boundary layer effects are then responsible for the consequent decrease of the dielectric constant.

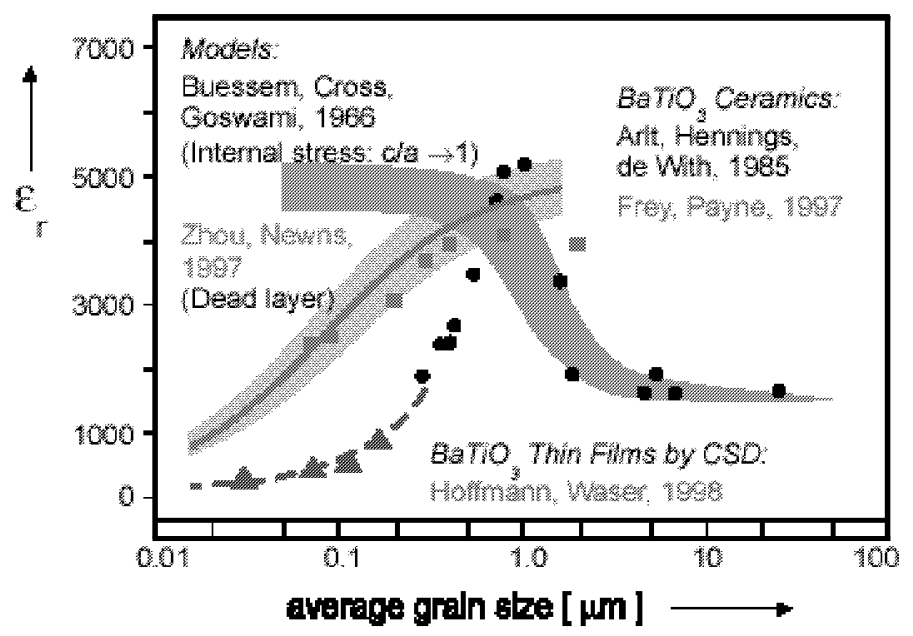

Fig. 2.8: Grain size dependence of the permittivity $\varepsilon_{r}$ measured at room temperature for BTO bulk-ceramics and polycrystalline thin films [31].

The observed decrease of the effective dielectric constant with decreasing film thickness could be caused by a so-called 'dead layer', i. e. a thin interfacial region with small $\varepsilon_{\mathrm{r}}$ which can be considered as a small capacitor in series [32]. This phenomenological model is supported by plotting the data on an inverse capacitance versus thickness plot (Fig. 2.9). For a simple capacitor this should result in a linear dependence with no offset. The observed offset value describes the interface capacitance. This approach will be discussed in detail for the films in the electrical properties section in Chapter 6. 


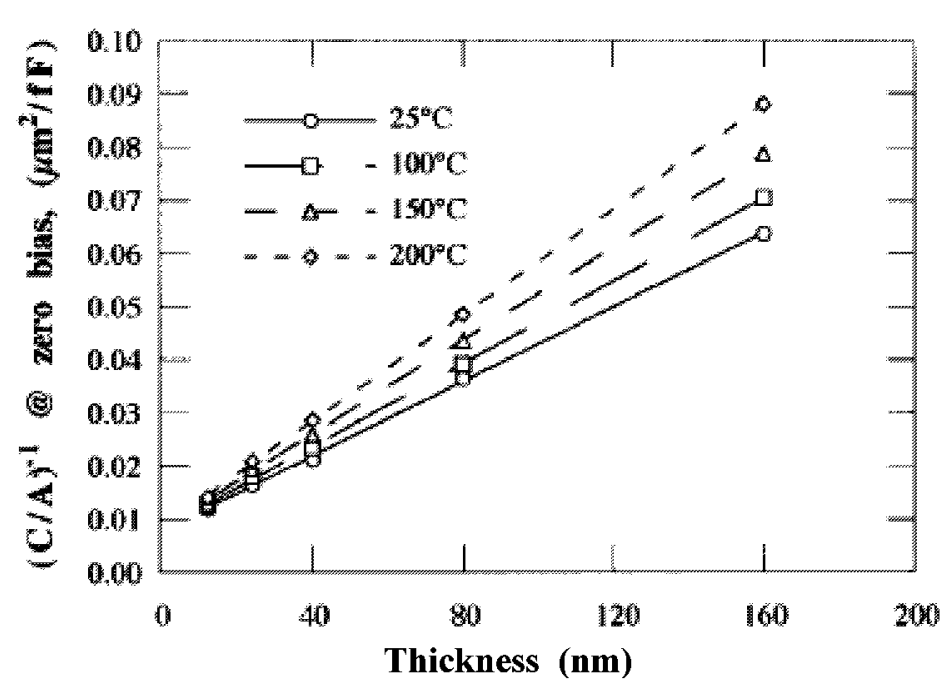

Fig. 2.9: Inverse capacitance area density vs. thickness plot for different temperatures. (MOCVD thin films with Pt electrodes) [35].

For the DRAM applications the observed flattening of the temperature dependence (Fig.2.7) of $\varepsilon_{\mathrm{r}}$ for thin films has the advantage of more stable operation and the disadvantage of a much lower and thickness dependent absolute value.

Current DRAMs have read pulses below 10ns which corresponds to $100 \mathrm{MHz}$ and these times will continue to decrease. Giannas and Basceri studied the frequency dispersion of the permittivity and the dissipation factor between $10 \mathrm{kHz}$ and nearly $20 \mathrm{GHz}$ (Fig. 2.10) [33, 34]. Basceri observed a constant dispersion of the capacitance over the measureable frequency range which was in the range of 5\%. The results documented in this thesis fully support these observations (see Chapter 6.4.2). The dissipation loss $(\tan \delta)$ appeared to increase above $10 \mathrm{GHz}$ by a factor of two, but later measurements pointed out that this could be due to nonideal measuring conditions. Concluding, it can be said that the frequency dependence of the dielectric constant of BST is not a limiting factor for its use in DRAM capacitors.

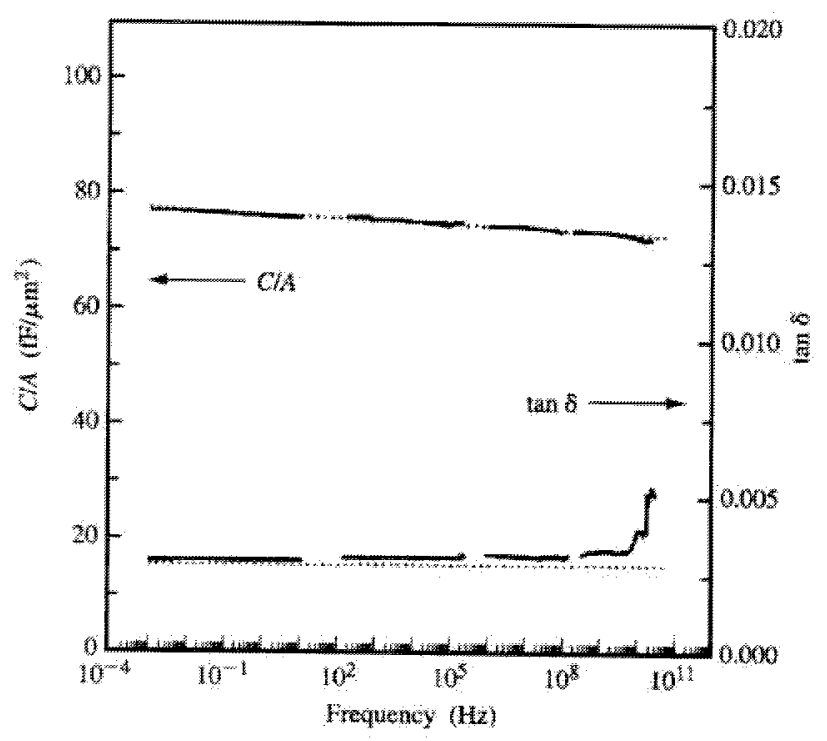

Fig. 2.10: Constant dispersion of the capacitance area density over 15 orders of magnitude. Dissipation loss remains almost stable over the same frequency interval [34].

An additional parameter of importance for DRAM application is the voltage dependence of the permittivity. Like many of the high-k materials BST is a non linear dielectric, i.e. the induced polarization displays a non-linear dependence on the applied field. This effect becomes also thickness dependent for thin films and is demonstrated in Fig. 2.11 [35]. This 
relation can be understood by the LDG-theory of ferroelectrics above the transition temperature [36]. As already mentioned, this transition is suppressed in thin films and the behavior is well described by this theory, too.

Fig. 2.11a displays the field dependence of the specific capacitance, e.g. the capacitance area density C/A, at room temperature over a thickness range from $24 \mathrm{~nm}$ to $160 \mathrm{~nm}$.
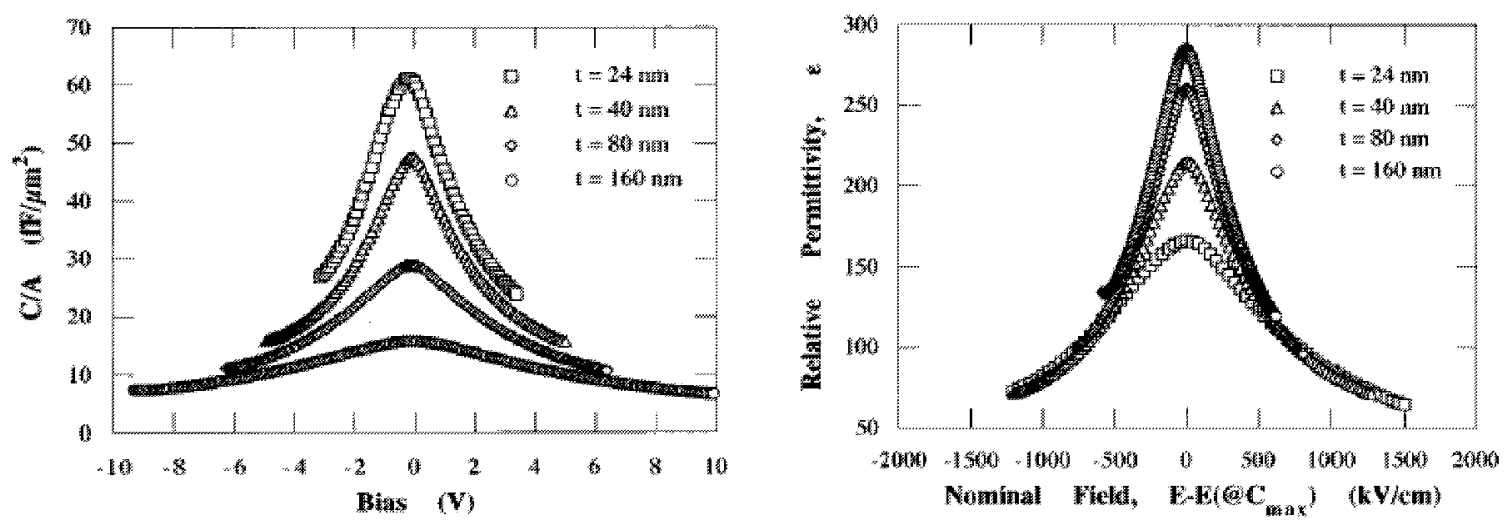

Fig. 2.11: a) Capacitance area density vs. bias voltage plot for samples of different thickness(C-V plot) b) Permttivity vs. electrical field plot yields not the expected similar trend for different thickness (MOCVD grown films) [35].

The capacitance increases with decreasing thickness as expected. For voltages $< \pm 1 \mathrm{~V}$, which are interesting for applications, the C/A values are above $30 \mathrm{fF} / \mu \mathrm{m}^{2}$, which is equivalent with a permittivity of approx. 250 at zero bias, and sinks dramatically $(<100)$ at voltages larger than $5 \mathrm{~V}$. This characteristic can be used in tunable devices and filters. The calculated relative permittivity (Fig. 2.11b) indicates the quantitative behavior more clearly and displays a decreasing maximum capacitance with decreasing thickness, while a constant maximum capacitance is expected. This behavior is an indication that the measured values are the effective ones and the previously mentioned 'dead layers', must be considered in a quantitative description. This approach will be discussed in detail for our films in Chapter 6.4. On the other hand it should be mentioned, that this flattening of the $\mathrm{C}-\mathrm{V}$ curve for thin films has also a small advantage for the application, as the total load of the capacitor at full voltage is less affected than the maximum permittivity at zero bias. 


\section{Fundamentals of the MOCVD for electroceramic thin films}

The use of MOCVD in electroceramic thin film deposition is associated with large investments from the semiconductor vendor side and a wide knowledge of the physicochemical processes is necessary in order to meet the requirements and the high standards set by the industry. A CVD system basically consists of three components (Fig. 3.1): the delivery for the chemical precursor molecules, the main reactor and the exhaust system which may be very elaborate for aggressive and toxic gases and byproducts. This chapter aims to present an overview of the MOCVD process from the beginning of nucleation at the hot substrate surface to effects that influence the film growth. In the second part of the chapter some general technological aspects regarding the equipment, e.g. reactor and delivery system, will be introduced.

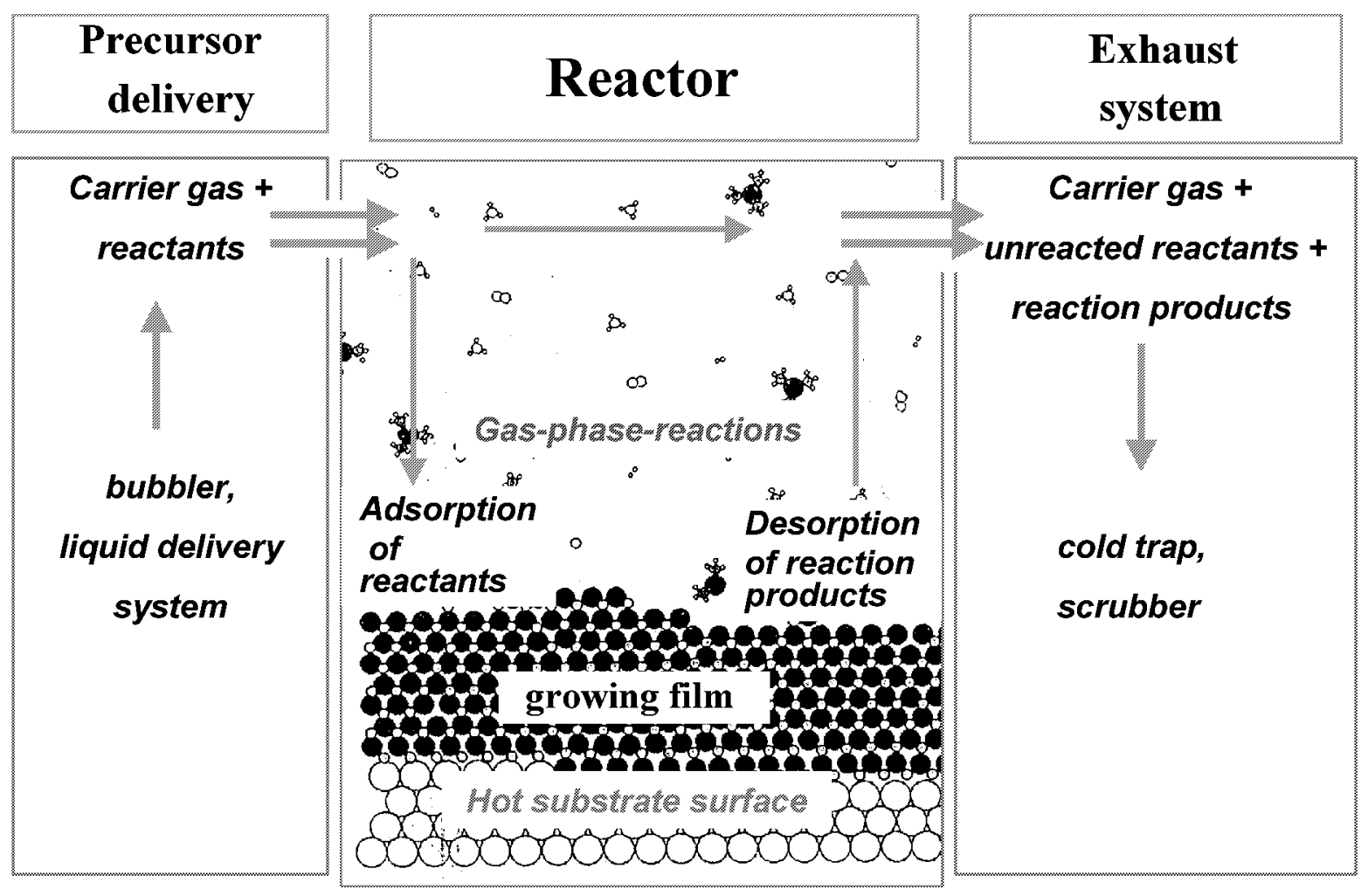

Fig. 3.1: Schematic of a CVD system consisting of precursor delivery, main reactor and exhaust system. Atomic scale mechanism of MOCVD growth in the reactor is indicated.

\subsection{The MOCVD process}

This chapter mainly deals with the basic physicochemical processes underlying chemical vapor deposition (CVD). CVD is a complex process consisting of many individual steps that often cannot be completely separated. Their interactions and the great number of chemical reaction steps, whose exact sequence remains mostly unknown, makes numerical simulation at present difficult. In addition, deposition by MOCVD usually proceeds under high supersaturation, i.e. far-off the thermodynamic equilibrium.

Growth is controlled by mass flow and/or reaction kinetics and a basic model of the process is depicted in Fig.3.1. Fig. 3.2 gives a closer look at the substrate surface, for the simple case of 
a horizontal reactor. In the horizontal reactor the wafer is placed parallel to the laminar flow direction and a velocity boundary layer forms over the susceptor. In the model from Schlichting it is assumed that the gas velocity $v(x, 0)$ over the susceptor is equal to zero and rises until it reaches a constant value $v\left(\mathrm{x}, \delta_{\mathrm{v}}[\mathrm{x}]\right)$ above the boundary layer [37]. The thickness of the boundary is determined by:

$$
\delta_{v}(x) \propto \sqrt{\frac{x}{\operatorname{Re}}}
$$

where Re is the Reynolds number.

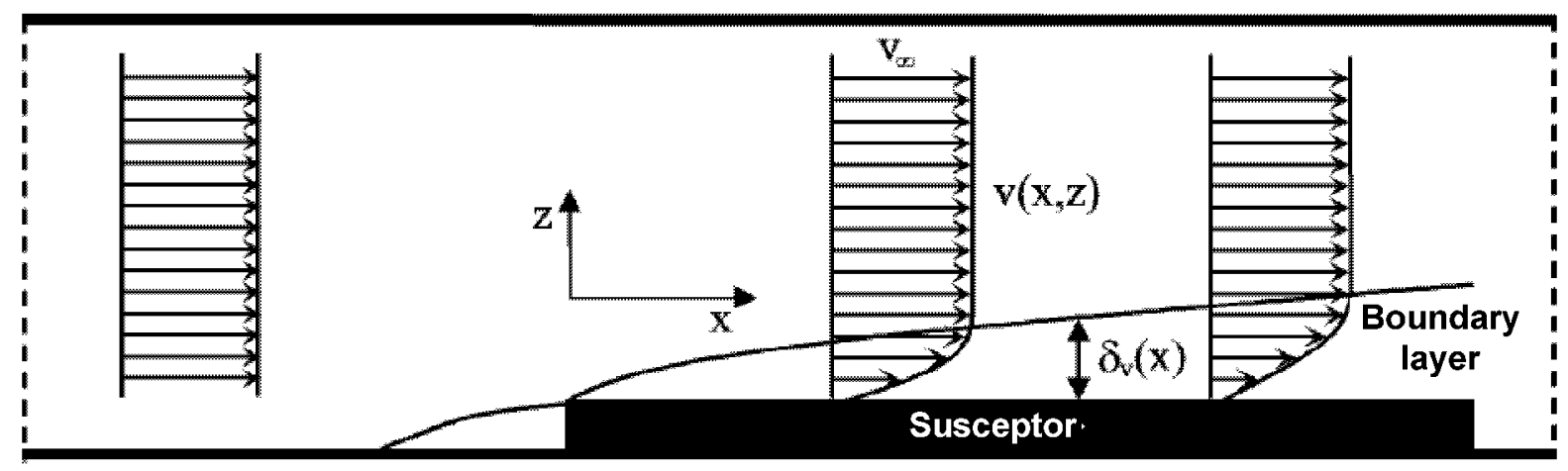

Fig. 3.2: Close look at the deposition region of Fig. 3.1. Boundary layer model with gas velocity profile in a horizontal flow reactor [38].

Due to the reactions at the hot surface, concentration gradients build up and the diffusion of reactants and/or reaction products through this boundary layer may be the limiting process for film growth. These conditions are generally described as diffusion limited reaction regime. If these processes cannot be clearly separated from the transport by gas flow, the more general term of (mass) transport limited growth is used.

It is obvious from this figure, that the thickness of the diffusion layer and concentration gradient varies over the substrate and no homogeneous film is obtained. A correction is necessary, e.g. by wafer rotation or by tilting the susceptor in order to change the velocity locally (see Chapter 3.2 for details). In the following, the major topics of the deposition and film growth process will be discussed in more detail.

\subsubsection{Nucleation and growth}

When injecting suitable precursors in a CVD reactor the film growth begins on the heated wafer surface and once the atoms of interest are released, the growth generally can be discussed analog to other atomic deposition methods. The composition and the microstructure of the film is strongly dependent on the nucleation processes on the growth interface and the surface diffusion, which are most dominantly influenced by the substrate temperature. Nucleation is initiated at energetically favorable spots on the substrate and even the most clean polished surface shows some structure. This is depicted schematically in Fig. 3.3 for a well polished single crystal surface with some characteristic features like terraces with length $1_{\mathrm{s}}$, steps and kinks in the step lines which run in well defined crystallographic directions. Fast diffusion at high temperatures leads the atoms to favorable places like steps and kinks assuring epitaxial layer by layer growth. This is the case if surface diffusion is faster than mass transport into the growth site meaning that adatoms have enough time to place 
themselves in the correct lattice position before they encounter other adatoms and form immobile clusters.

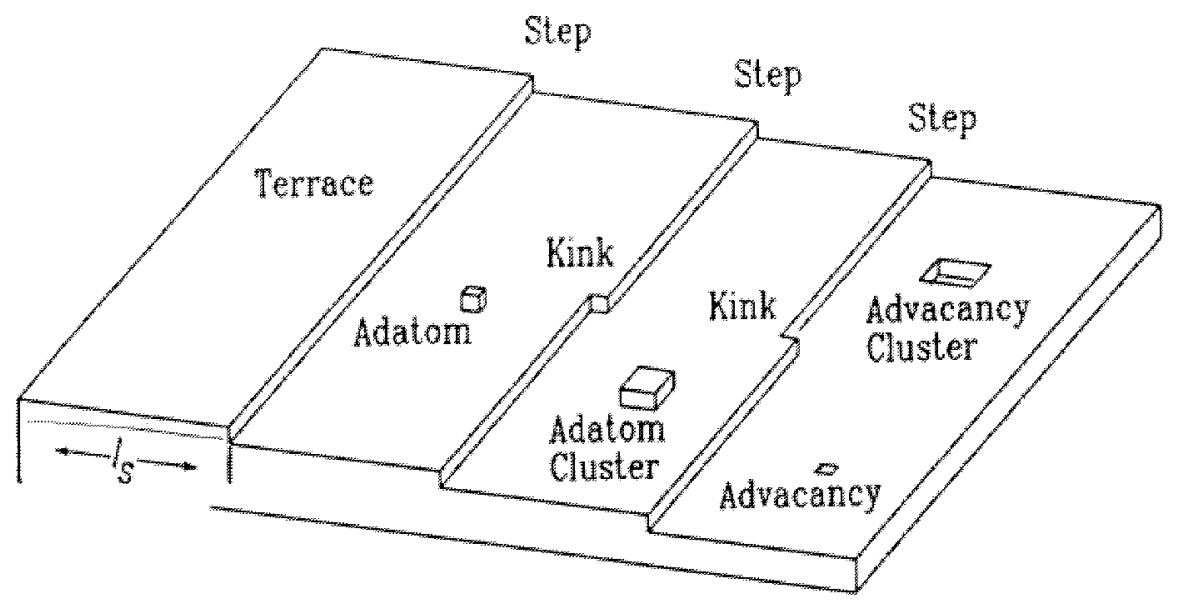

Fig. 3.3: Elements of surface morphology. Step growth in levels (terraces) formed by adatom clusters is depicted. Each terrace has the length $l_{s}$ [14].

On the other side, we may obtain island growth at low temperatures if the surface diffusion is much slower than the mass transport. Several mobile adatoms may then encounter within a terrace and build immobile clusters. After the terrace is covered with such clusters, many adjacencies may have been formed or even built own clusters. At very low temperature the atomic arrangement to the equilibrium crystal structure may even be too slow and amorphous films may be deposited.

So far we did not consider differences between the film and the substrate material, which corresponds to the simplest case of homo-epitaxy. The more general case is the growth on a different material, hetero-epitaxy. The most important material parameters controlling nucleation and growth are the surface energy (surface tension) and the crystal structure (or simply the lattice parameter). For materials with the same structure but different lattice parameters we refer to pseudomorphic growth and can obtain defect-free, but highly strained layers (see Chapter 3.1.2). As illustrated in Fig. 3.4, there are three general modes of CVD film growth.

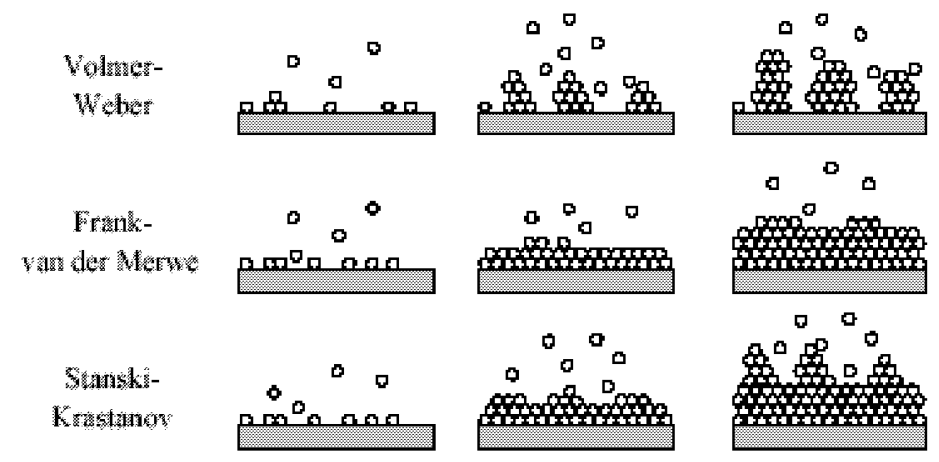

Fig. 3.4: Possible types of growth modes: (top down) a) Island or Volmer-Weber growth b) Layer-bylayer or Frank-van der Merwe growth c) layer-island or Stanski-Krastanov growth [39].

The 3D island growth is often referred to as Volmer-Weber growth. Small clusters are nucleated on the substrate surface where they finally grow into islands which eventually 
coalesce to form a continuous film (Fig. 3.4a). We obtain such growth if the film atoms are more strongly bound to each other than to the substrate. Thus, in terms of the surface energy $\gamma$, we can write:

$$
\gamma_{\text {layer }}+\gamma_{\text {substrate/layer }} \geq \gamma_{\text {substrate }}
$$

In the opposite case, known as Franck-van der Merwe growth, two-dimensional layer-bylayer growth takes place (Fig. 3.4b). In this case the bonds between the atoms are not as strong as to the substrate. In order to observe perfect layer by layer, growth kinetics must be considered similar to the case of homo-epitaxy, and slightly off-axis $\left(1-3^{\circ}\right)$ oriented substrates may be used to reduce the distance between nucleation sites and suppress island growth.

The Stranski-Krastanov growth mode (Fig. 3.4c) is a combination of the two other modes. After the growth of one or a few monolayers, there is a transition from the layer growth mode and islands growth starts on top of the initial layers. This transition may be energetically favored if there is lattice mismatch between substrate and film.

For the growth on polycrystalline substrates, as it is considered in this work for BST on Pt electrodes, these mechanisms can be considered only locally, i.e. on different grains of the substrate. Grain boundaries will arise if a closed film forms by coalescence of different oriented grains.

\subsubsection{Strain and relaxation}

Strain energy release is one of the possible reasons for the transition from layer-by-layer growth into island formation in the Stranski-Krastanov mode. This can be explained according to Eq. 3.3 which is illustrated in Fig. 3.5a [14]. Whereas the surface energy increases with the island size the strain energy increases proportional to the film volume and makes island formation more favorable with increasing thickness:

$$
\Delta \mathrm{E}=\mathrm{E}_{\text {surface }}+\mathrm{E}_{\text {relaxation }} \approx \mathrm{A} \cdot \gamma \cdot x^{2}-\mathrm{B} \cdot k \cdot \mathcal{E}^{2} \cdot x^{3} \quad \text { Eq. } 3.3
$$

$x$ represents the island size, $k$ the bulk modulus and $\varepsilon$ the strain; A and B are constants. A simple model of the strain relaxation, which is possible for an island and not for a closed film, is plotted in Fig. 3.5b for the case that the film has a larger bulk lattice parameter than the substrate.

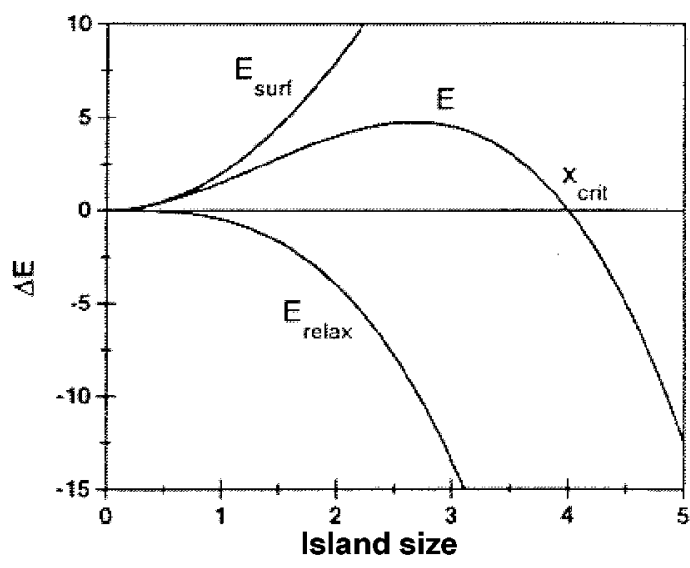

Fig. 3.5a: Energy contributions as a function of the island size.

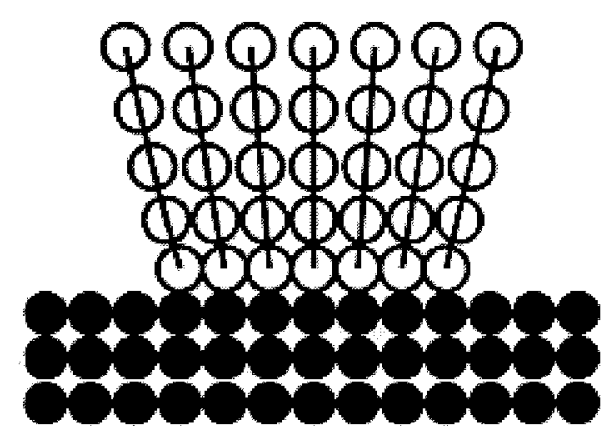

Fig. 3.5b: Strain relaxation of an island which is rigidly bound to the substrate. 


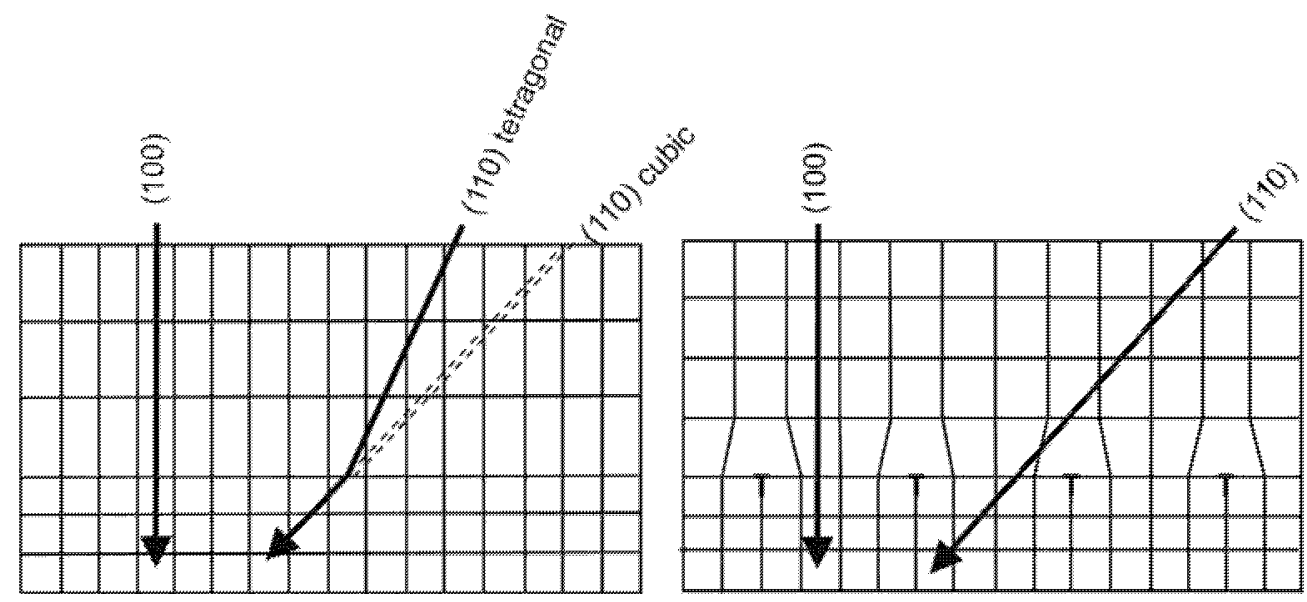

Fig. 3.6: Strain relaxation by misfit dislocations.

As the islands overlap during film growth and form a closed film, the lattice can no longer relax in this manner. The strain can now relieve due to formation of misfit dislocations as depicted in Fig.3.6. Thin films may start with perfect epitaxial growth relative to the substrate having their unit cell tetragonal distorted. A lower in-plain lattice parameter causes an expansion in the direction normal (perpendicular) to the plain, according to Poisson's number. This is also expressed by a tilt of the crystallographic angles as shown in Fig. 3.6 for an initially cubic lattice. Strain relaxation forces the formation of dislocations taking the unit cells nearly back to their initial cubic form. Similarly two dimensional defects, like twins or anti-phase boundaries, may be formed and are shown in Chapter 6.

\subsubsection{Thermodynamics and kinetics}

Film growth processes do not take place in an exact equilibrium state and are rather driven by the thermodynamic force towards an equilibrium. Therefore, the partial pressures of the reactants in the gas phase above the substrate must be higher than in the equilibrium state. As the precursor supply is constant, the equilibrium cannot be established and this drives the CVD process. This so called supersaturation of the gas phase is limited by kinetics and the maximal possible mass transport to the substrate.

CVD processes are usually driven by thermal energy. The temperature can be coupled on the substrate surface by many ways, e.g. by resistive heating, IR lamps or RF heaters, and causes the decomposition of the precursors that leads to film formation. Fig. 3.7 shows a typical growth rate dependence on substrate temperature at a constant flow rate for the example of polysilicon growth from a $\mathrm{SiCl}_{4}$ precursor. Three growth temperature regions are visible.

The growth rate $\mathrm{j}$ at low temperatures is limited by chemical kinetics (kinetically limited region, $\left.\mathbf{j}_{\text {kinetics }}\right)$ and increases exponentially with temperature according to the Arrhenius expression in Eq. 3.4. Actually, reaction kinetics include a great number of different process steps from precursor reaction to film growth kinetics, so that the limiting step is not known and an effective activation energy $E_{A}{ }^{\text {eff }}$ is generally accounted.

$$
\mathrm{j}_{\text {kinetics }}=\mathrm{A} \exp \left(\mathrm{E}_{\mathrm{A}} / \mathrm{RT}\right)
$$

where $E_{\mathrm{A}}$ is the apparent activation energy, $R$ the gas constant, and $T$ the temperature. 


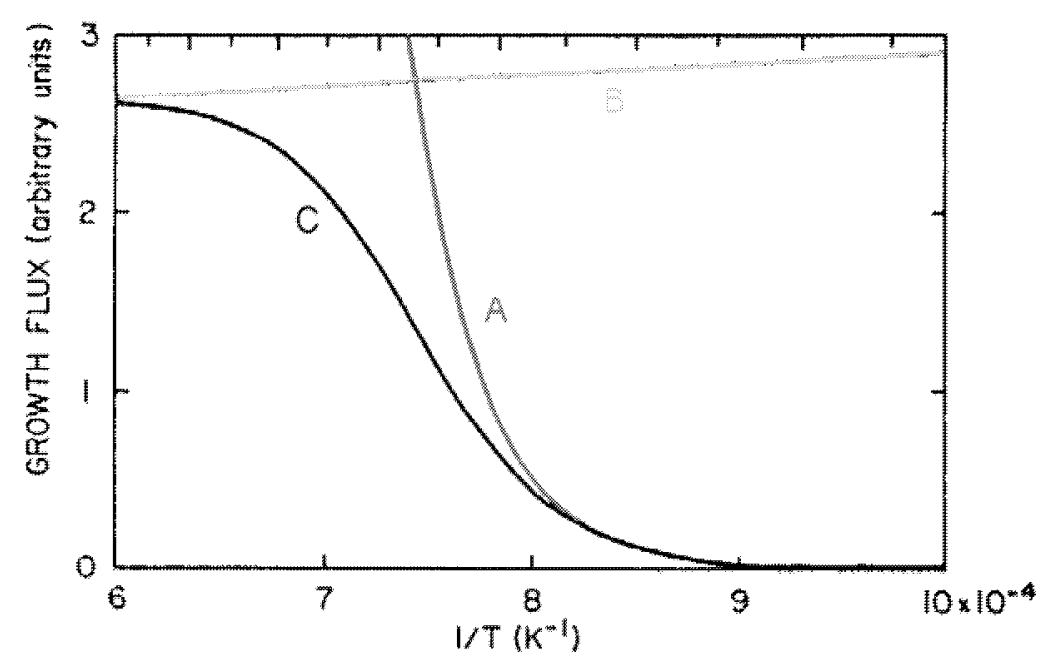

Fig. 3.7: Typical deposition rate variation with reciprocal growth temperature based on the growth of polysilicon from $\mathrm{SiCl}_{4}$ [40].

A: kinetic controlled reaction

$B$ : transport controlled reaction

$C$ : resulting experimental observation

In this regime, the deposition rate depends just weakly on the flow homogeneity and this region is therefore best suited for conformal deposition (see section 6.1.2.f). However, temperature variations have to be minimized to ensure uniform film thickness. Additionally, the incorporation rate of the precursors decreases fast with temperature. The decrease may be different for each precursor species. Consequently, there is just a narrow process window to achieve good conformality on complex $3 \mathrm{D}$ structures and acceptable deposition rates for mass production.

The exponential increase of the growth rate cannot go on at the intermediate and high temperature regime, as the mass transport to the surface becomes a limiting process (masstransport-limited region, $\mathrm{j}_{\text {transport }}$ ). Assuming a limitation by the diffusion in the gas phase, the growth rate is given by:

$$
\mathrm{j}_{\text {transport }}=\mathrm{B} \mathrm{T}^{-1 / 6}
$$

Hence, there is a very weak temperature dependence, but the process is more sensitive to the stability of the flow pattern. Since the flux of the reactants to the surface is proportional to the precursor concentration, one can simply adjust the magnitude of the growth rate by the flow rate.

Generally, the growth modes cannot be clearly separated and there is a superposition of the kinetic and mass-limited regions in most CVD processes. The overall growth rate $j_{\text {total }}$ is obtained by adding the two reciprocal fluxes which can be considered as two resistive elements is series.

$$
1 / \mathbf{j}_{\text {total }}=1 / \mathbf{j}_{\text {kinetics }}+1 / \mathbf{j}_{\text {transport }}
$$

The growth rate often shows a further decrease at high temperatures. The reason is possible pre-reactions that take place in the gas phase. These reactions may lead to nucleation centers causing cluster formation. This is a major concern in the industry, since this effect leads potentially to inhomogeneities and particles on the film. Additionally, pre-deposition on the reactor walls causes increased reactor maintenance and may affect the film growth process. 


\subsubsection{Transport phenomena in the gas phase}

Transport phenomena (i.e. liquid flow, heat and mass transfer) control the transfer of the precursors to the substrate and influence the degree of desirable and unwanted gas-phase reactions taking place before deposition. Due to the complex reactor geometries ranging from inlet nozzles to exhaust manifolds and the thermal gradient characteristic of modern CVD reactors (see Section 3.2.2), severe problems arise in the simulation and visualization of the characteristic flow patterns which impact the process stability. Additionally, the process simulation is complicated by the fact that the phenomena take place at different length scales. Some of the phenomena that need to be simulated and the scale where they occur are presented in the following:

- macro-scale: turbulences, hot or cold spots

- micro-scale: conformal deposition

- atomic-scale: chemical reaction, nucleation and growth, desorption

The transport process in a reactor is usually characterized by dimensionless parameter groups depending on reactor properties, like geometry, and the reactants, e.g. density, heat conductivity and viscosity. These parameters help to approximate the transport mechanisms in new reactor designs and optimize present systems using insights gained from modeling and simulation. The Knudsen number $(K n)$ describes the behavior of molecules in a gas flow. If $K n$ is small $(<0.01)$ the molecules undergo gas-phase collisions and a continuum description is efficient. This is true for large reactors that operate at high pressures, that is the mean free path is small relative to the characteristic reactor dimensions. At the other extreme $(\mathrm{Kn}>10)$ wall collisions dominate in the system. The later case is typical in vacuum epitaxy systems for silicon and compound semiconductors. Low pressure CVD systems operate rather in the transition regime between continuum behavior and free molecular flow. The transition and free molecular regimes are of great importance while growing on micron-sized features.

A laminar flow pattern is essential for high process reproducibility that leads to higher yields as demanded by the semiconductor industry. This is expressed by the Reynolds number $(R e)$. $R e$ should be low enough $(R e<100)$, also meaning low gas velocities, to ensure that the flows are laminar. This is typically the case in conventional CVD reactors. Nevertheless, the gas flow in large diameter multiple wafer rotating disk reactors may be turbulent as the rotational Reynolds number $\left(R e_{\Omega}=\omega R / v\right)$ becomes very large.

The relative contributions of convection and diffusion to the mass and heat transfer are given by the Peclet numbers $(P e)$. Convection dominates for large Peclet numbers $(P e>10)$, diffusion for small values $(P e<1)$. This is very useful to estimate the level of impurities incorporation in the film.

The natural convection in the system is approximated by the Grashof $(G r)$ and Rayleigh $(R a)$ numbers. Convection phenomena are mainly generated from the density variation of the process gases $(V=n R T / p)$ due to temperature gradients. The depletion of reactants by film growth cannot actually create significant density variations in the fluid. $R a$ is calculated by the product of the Prandl $(P r)$ and Grashof numbers, where $P r \approx 0.7$ for CVD gases.

The Damköhler numbers $(D a)$ express the time available for transportation relative to the reaction time. This is also known as CVD number. A large $D a$ means mass transfer limited growth, whereas for small $D a$ numbers the growth is limited by the surface kinetics [20].

As described by the Grashof-Rayleigh numbers, the temperature variations and occasionally the concentration gradients produce buoyancy flows that superimpose on the flow entering the reactor. This effect may have a significant impact on the film thickness and composition 
uniformity, as well as on impurity incorporation. In a vertical reactor, the buoyancy force opposes the incoming gas stream, while it is perpendicular to the gas flow in the horizontal reactor geometry.

For simple axisymmetric flows in vertical reactors it is found that the ratio of natural convection to forced convection varies as $G r / R e^{2}$. However, it is very difficult to develop a single criterion valid for a broad range of reactor configurations because of the nonlinear nature of the mixed convection flow and the many boundary conditions, e.g. thermal gradients and reactor walls. Later simulations by Evans and Greif on rotating disk reactors showed a stable axisymmetric flow for process and reactor parameters leading to $\mathrm{Ge} / \mathrm{Re}^{3 / 2}<6$. However, even if the reactor shape and final temperature distribution is axisymmetric, a nonaxisymmetric flow field may still result from azimuthal temperature disturbances during reactor start-up [20].

In spite of all difficulties, numerical simulations taking into account the transport phenomena in the reactor are helpful to initially determine a larger process window, before the optimal parameter set is extracted through intensive process optimization. As an example, we show some simulations of flow patterns for an AIX 2400 Planetary Reactor ${ }^{\circledR}$, that were accomplished in the preliminary design stage of our reactor. This reactor type can handle five 4 " wafer simultaneously. The gas inlet nozzle is a critical part for the gas flow and two typical nozzle types for oxide deposition are depicted in Fig.3.8a and Fig.3.8b, respectively. The cone injector is the most simple nozzle type, however, much better results are achieved with a nozzle, which ensures better mixing in the gas phase and increases the deposition efficiency as shown in Fig. 3.9a, that presents the distribution of the gas velocity immediately after the nozzle outlet.

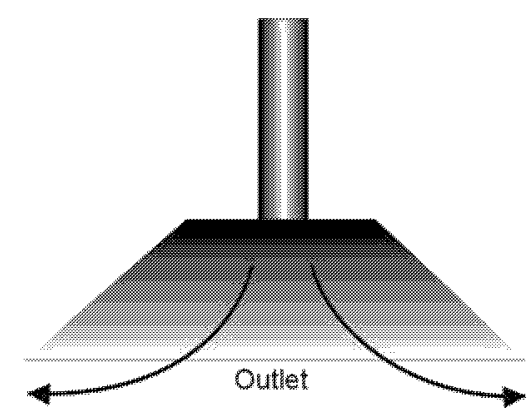

Fig. 3.8a: Simplest nozzle design: cone.

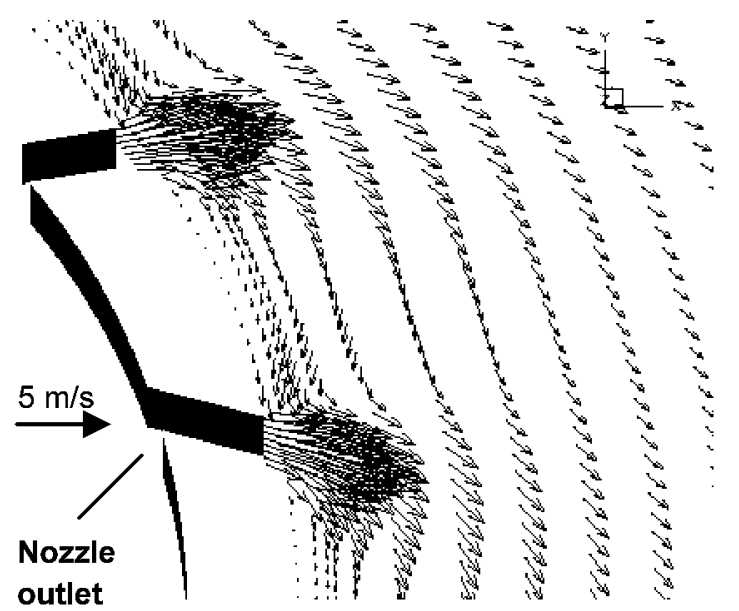

Fig. 3.9a: Simulated gas velocities immediately after the nozzle outlet (top view) [41].

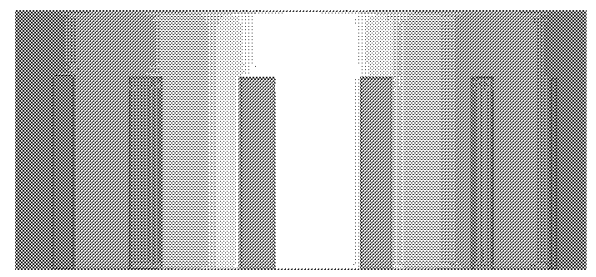

Fig. 3.8b: Schematic of the applied nozzle.

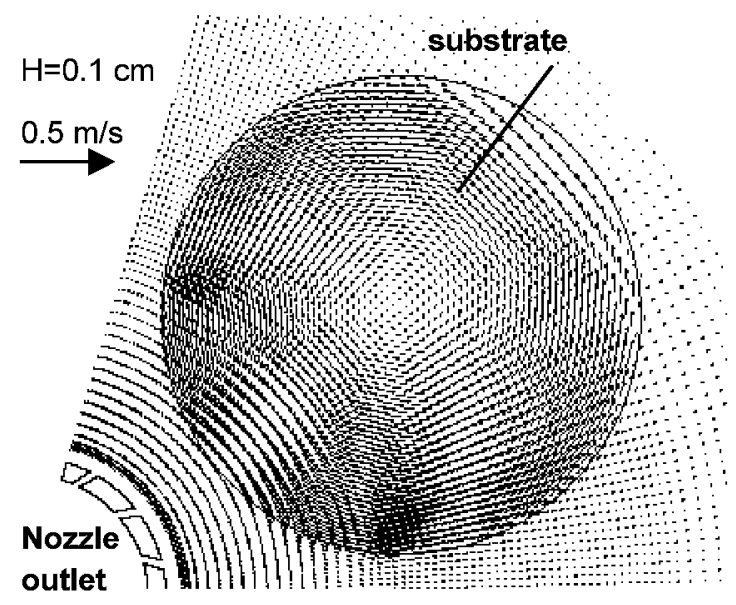

Fig. 3.9b: Influence of the rotating satellite $(\omega=60 \mathrm{rpm})$ on the gas vepocity profile [41]. 
Fig. $3.9 \mathrm{~b}$ displays the influence of the rotating satellite on the gas velocity on a slice at $1 \mathrm{~mm}$ distance from the susceptor. The rotational flow almost disappears beyond a vertical distance of $\sim 5 \mathrm{~mm}$. Although the lateral dimensions of this reactor are approx. $30 \%$ smaller than in the AIX 2600G3 reactor (reactor height: 20\%), the flow patterns may be considered similar. Initially, the simulation was performed at $100 \mathrm{mbar}$ [41]. Consequently, the magnitude of the gas velocity $1 \mathrm{~mm}$ above the susceptor at $2 \mathrm{mbar}\left(\mathrm{T}_{\text {growth }}=650^{\circ} \mathrm{C}\right)$ must be $\sim 50$ times higher, as long as the mass flow is kept constant. This results in velocities in the range of $25-250 \mathrm{~m} / \mathrm{s}$ [42].

A further example for the advantage of the susceptor rotation offers the simulation by Fotiadis shown in Fig. $3.10 \mathrm{a}-\mathrm{b}$ [20]. The generation of a high speed rotation $>500 \mathrm{rpm}$ helps to create a uniform mass transfer layer over the susceptor. Hence, convection driven recirculations can be avoided without having to increase the inlet flow rate. Besides improved uniformity the rotation also leads to higher growth efficiency compared to conventional showerhead reactors (see also Chapter 5.2). EMCORE has applied this principle in its TurboDisc reactor series [43].

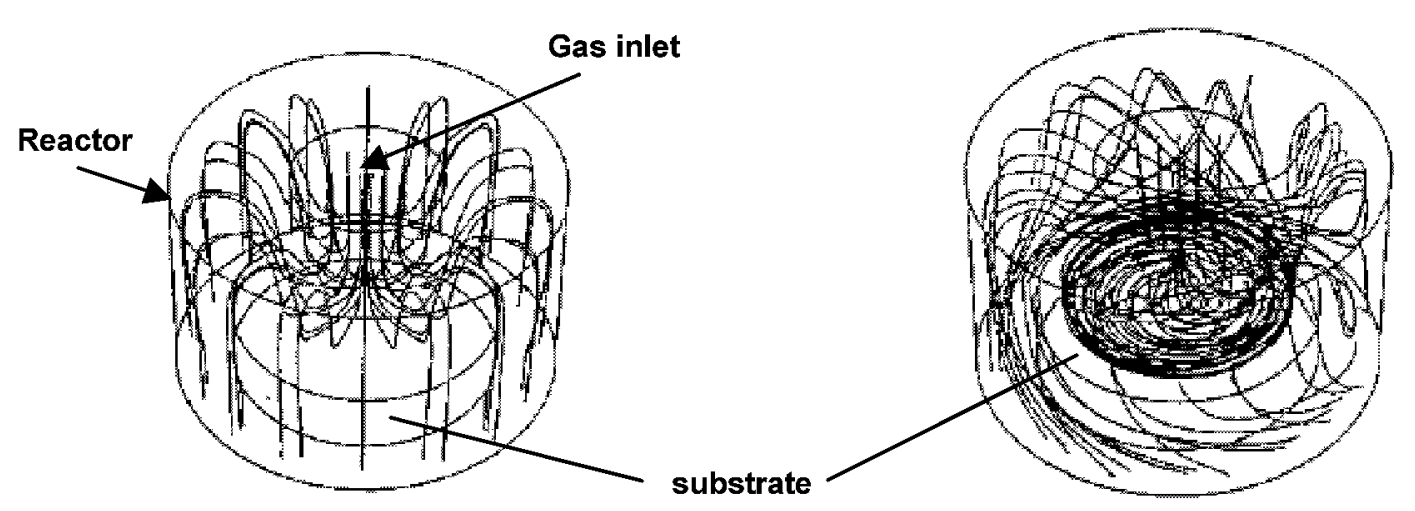

Fig. 3.10: Gas flow patern in a vertical reactor before (a) and after susceptor rotation at high speeds (b). Simulation by Fotiadis [20].

The reactor geometry has also a major impact on the flow pattern. Especially the distance between the inlet and the susceptor, which enters the Grashof number as $L^{3}$, is clearly a critical parameter in preventing thermal recirculations. However, the minimal distance from the heated susceptor is limited because of predeposition phenomena in the nozzle. A larger nozzle potentially improves film thickness by distributing the precursors over a wider substrate area, but the decrease in linear flow velocity and consequently the Reynolds number can cause intensive natural convection phenomena.

The thermal stability is extremely important in CVD reactor operation. CVD reactors may operate in a region controlled by surface reaction processes in order to achieve conformal deposition of sub-micron features, and a few degrees variation in surface temperature can lead to unacceptable variations in the step coverage. More often, CVD systems operate often in the mass transfer limited range so that small susceptor temperature variations have little effect on the growth rate. Nonetheless, the flow pattern may be strongly influenced by thermal instabilities resulting in film thickness and composition uniformity variations.

Finally, it is often desirable to operate CVD reactors at reduced pressures unless this is not restricting for the chemistry. This is also depicted in the Rayleigh and Grashof numbers which are directly proportional to the square of the gas density. Consequently, natural convection 
phenomena decrease strongly with lower pressure. Moreover, the residence time of the gases in the system is reduced, thus minimizing parasitic gas phase reactions that cause impurity incorporation or gas-phase nucleation.

\subsubsection{Low pressure CVD}

In addition to the previous arguments, the reactor pressure is an important parameter to control, as it determines the free path length of the atoms or molecules, which affects the reaction probability in the gas phase and also the conformal deposition. In a first approximation, the free path length $L$ may be expressed by Eq. 3.7, where atoms are considered as interacting masses with a Maxwell velocity distribution; $n$ is the concentration of gas and $d$ stands for the diameter of the molecule:

$$
L=\frac{1}{\pi \sqrt{2} \cdot n \cdot d^{2}}
$$

Using Eq. 3.7 we obtain at $0.510^{-3} \mathrm{mbar}$ a free path length of $20 \mathrm{~cm}$ for air, which is a typical reactor distance. Similarly, we obtain the number of gas atoms that hit the film surface at a certain temperature $T$ :

$$
n=p \sqrt{\frac{1}{2 \pi \cdot k_{B} \cdot m \cdot T}}
$$

$m$ is the atomic or molecular mass, $k_{B}$ the Boltzmann constant and $p$ the partial pressure of the atoms in the gas phase.

It is obvious that the mean free path length can be important in case of conformal deposition in small structures like vias or trenches with high aspect ratios that are commonly used in VLSI technology, Fig. 3.11.

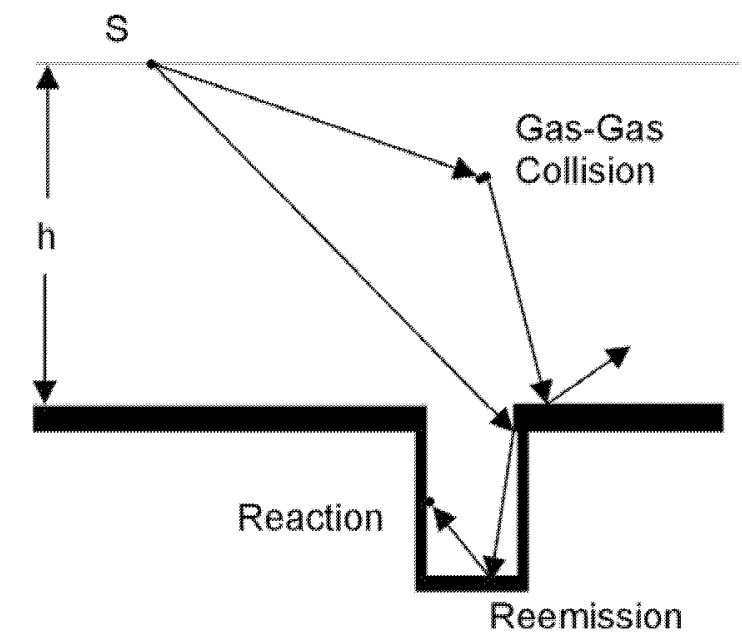

Fig. 3.11: Deposition into a trench of smaller dimensions than the mean free path length of the gases. Reflection of the precursor molecules at the walls becomes important.

As the mean free path length is much higher than the sub-micron dimensions of such structures, gas collisions in these structures become unlikely even for medium pressure conditions making atom reflection on the walls more important and Monte Carlo methods have to replace the gas continuum theory for the simulation of the deposition process. 


\subsection{CVD reactor systems}

CVD systems must be designed and operated in such a manner that film thickness, crystal structure, surface morphology and composition can be accurately controlled. A CVD reactor system basically consists of a precursor preparation unit for delivering the source compounds, a reactor unit and an exhaust system, Fig. 3.1. The major role of the precursor chemicals will be discussed first, followed by a short description of the most important delivery systems. Finally, commercially available reactor types will be presented and their suitability for growth of electroceramic thin films will be discussed.

\subsubsection{Chemical precursors and delivery}

\section{a) Precursor chemistry}

In order to fulfill the requirements for ultra large scale integration (ULSI) of ceramic films suitable precursors play a major role. Unfortunately, a great number of precursors necessary for deposition of ceramic materials like BST, SBT and PZT are characterized by low volatility and low thermal stability. This is the reason why huge efforts have been invested in the development of metal precursors, especially for the group-II metals, e.g. in form of organo-metallic compounds.

Since different precursors are used for deposition of multi-component systems like BST, all metal precursors must have a sufficient high vapor pressure in the same order of magnitude, in order to allow vapor-phase mixing and transport of the precursor molecules. A vapor pressure of $0.1 \mathrm{mbar}$ at $100^{\circ} \mathrm{C}$ is considered the lower limit. Molecular stability in the gas phase is also a major concern in order to prevent premature reaction and decomposition of the precursors during vapor-phase transport. These properties as well as a sufficient large temperature margin between vaporization and decomposition define a narrow process window for the deposition of ceramic films. Additional storage, maintenance and environmental requirements like long term stability, low moisture sensitivity, complete decomposition and toxicity display the complexity of the process and the importance of suitable precursors.

The currently used metal-organic precursors are summarized here under the term MOCVD precursors, although they sometimes include compounds that are more specifically referred as organometalic precursors (OMCVD). MOCVD precursors comprise metal-alkyles, metalalkoxides and metal- $\beta$-diketonates. Metal-alkyles can be summarized under the general formula $M^{n+} R_{n}$, where $M$ represents the metal and $R$ a hydrocarbon chain of the form $\mathrm{C}_{\mathrm{m}} \mathrm{H}_{2 \mathrm{~m}+1}$. An example of a metal-alkyle precursor is tetra ethyl lead (TEL) which is described as $\mathrm{Pb}\left(\mathrm{C}_{2} \mathrm{H}_{5}\right)_{4}$. The advantages of metal-alkyles are the rather high vapor pressures at low temperatures. They are usually in the liquid phase at room temperature. Unfortunately, their high toxicity as well as moisture and oxidation sensitivity make them hard to handle.

Metal-alkoxides of the general form $\mathrm{M}^{\mathrm{n}+}(\mathrm{OR})_{\mathrm{n}}$, are distinguished from the alkyles by an additional oxygen atom coupling the metal with the hydrocarbon chains. An example for an alkoxide is titanium iso-propoxide (TIP) or $\mathrm{Ti}\left(\mathrm{O}^{\mathrm{i}} \mathrm{Pr}\right)_{4}$. Such precursors combine higher stability with lower volatility and toxicity compared to the alkyle compounds. 


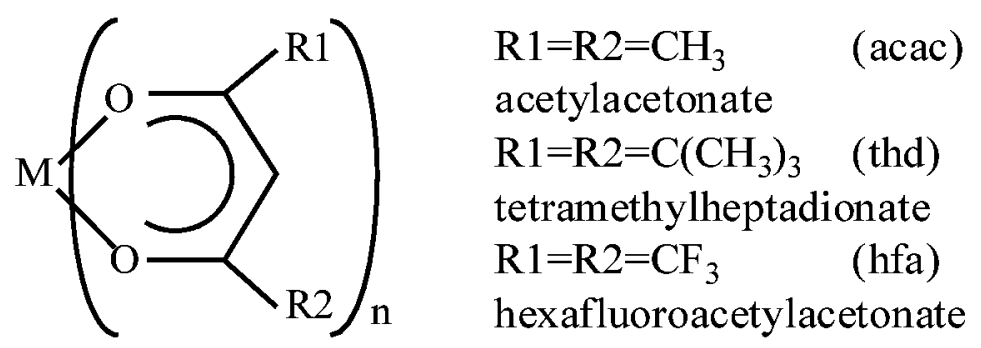

Fig. 3.12: Structure of a $\beta$-diketonate molecule with the most important ligands (RI and R2). $M$ represents the metal ion.

The $\beta$-diketonates are currently the most important precursors of group-II metals for the MOCVD technology. Their structure is depicted in Fig. 3.12. The ligands $R_{1}$ and $R_{2}$ may consist of diverse alkyles and have a great influence in the precursor stability and volatility. Hence, the properties of $\beta$-diketonates are adjustable within a broad spectrum through the appropriate choice of ligands, e.g. the volatility rises with higher volume and fluorine content of the ligands. In both cases the molecular interaction is reduced. The reason is that the increased ligand volume acts as shielding and protects the metal ion from reacting with its environment. In the case of fluorine, the shielding effect is provided by its higher atomic radius compared to hydrogen. In both cases, the stability is also improved by prohibiting oligomerization or decomposition because of moisture or oxygen influence. Their volatility and stability may be enhanced further by the use of adducts like tetraglymes and pmdeta [44].

In order to increase the compatibility with other precursors the composition of mixed precursors such as $\mathrm{Ti}\left(\mathrm{O}^{\mathrm{i}} \mathrm{Pr}\right)_{2}(\mathrm{thd})_{2}$ is possible. The use of this precursor instead of the pure Tialkoxide is that ligand exchange with other $\beta$-diketonates, e.g. $\mathrm{Ba}(\text { thd })_{2}$, does not take place. Otherwise this would lead to nonvolatile Ba-alkoxide formation, which would clog the vaporizer system.

\section{b) Delivery systems}

The delivery system meters and mixes the precursors to be used in the reactor unit. The design depends on the source compounds. The classical delivery system for precursors which are stable in liquid form is the 'bubbler'. In a bubbler, an inert carrier gas is led through the liquid precursor, as shown in Fig. 3.13. The generated bubbles rise and transport vapor to the surface.

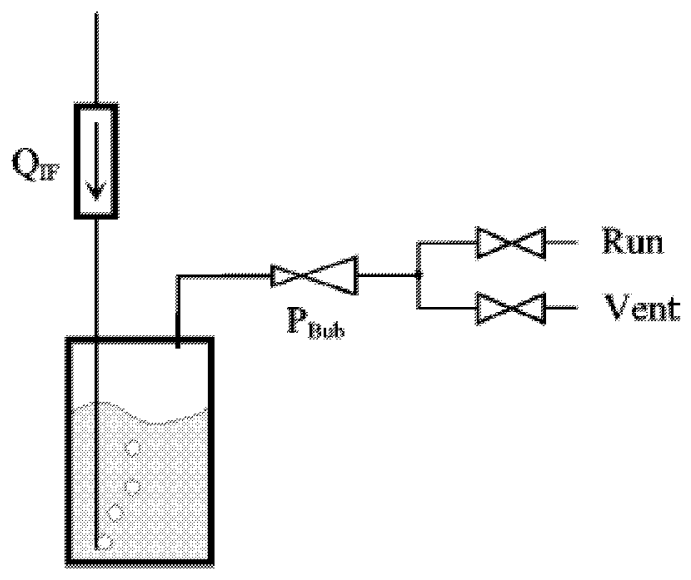

Fig. 3.13: Bubbler operation principle. The Bubbler pressure and the carrier flow are controlled separately. 
The mixture of carrier gas and precursor vapor above the liquid surface is led directly to the reactor. The amount of precursor transferred to the reactor is determined by the source temperature, which determines the equilibrium vapor pressure, and the carrier gas flow rate. The flow rate of the precursor $\left(F_{\mathrm{p}}\right)$ given in $\mathrm{sccm}$ (standard $\mathrm{cm}^{3}$ per minute) or $\mathrm{mol} / \mathrm{min}$ is:

$$
F_{\mathrm{p}}=F_{\text {carrier }} \cdot P_{\mathrm{p}} /\left(P_{\text {total }}-P_{\text {carrier }}\right) \quad \text { Eq. } 3.9
$$

where $P$ is the partial pressure. The index $p$ refers to the precursor and $c$ to the carrier gas. For stable temperatures, the flow rate can be controlled by the carrier gas flow. Therefore, the transfer lines to the reactor must always be kept above the bubbler temperature to avoid condensation and ensure process stability.

The group-II metal precursors are solid up to high temperatures with low vapor pressures, e.g. $0.05 \mathrm{mbar}$ at $200^{\circ} \mathrm{C}$ for $\mathrm{Ba}$ (thd $)_{2}$ and $0.2 \mathrm{mbar}$ at $230^{\circ} \mathrm{C}$ for $\mathrm{Sr}(\mathrm{thd})_{2}$. The precursors are not very stable at these temperatures, where neither direct sublimation nor the dissolution in a proper solvent yields a controlled process. Therefore, liquid delivery systems have evolved where the precursors are dissolved into an appropriate solvent and evaporated close to the reactor. These systems combine many advantages compared to the conventional bubbler principle. The precursors can be metered, mixed and transported to the vaporizer at room temperatures in the liquid phase. The thermal load remains low and premature decomposition and aging effects can be avoided.

Different evaporation techniques have been developed: flash and contact free evaporation. In the first case of flash evaporation the liquid precursor mixture is immediately evaporated by hitting a hot surface and then transported with a hot carrier gas into the reactor. In case of a contact free evaporation mode, the solution enters as droplets or aerosol in a heated zone, where it slowly evaporates. Contact vaporizers have the major drawback, that residues form on the hot plate and the vaporizer region and this can lead to clogging of the lines and irreproducible results. This can be avoided by contact free systems using e.g. ultrasonic nebulizers where the precursor liquid is first transformed to an aerosol. This consists of droplets of well-defined diameter that are initiated in a hot carrier gas stream and transported to the reactor. The increased effective surface of the aerosol particles in relation to the surface in the liquid phase leads to a complete contact free evaporation of the precursors.

Different contact free vaporizers have been used on a laboratory scale using single solution cocktails for the deposition of several elements and have been discussed in the Ph.D. thesis by Schäfer [39]. The system basically consists of a supersonic nebulizer, the carrier and process gas supply and a whirl chamber where a sufficient mixing of the gases is achieved in order to obtain a homogenous velocity profile through the vaporizer tube and at the inlet to the reactor. The drawback of the system is the limitation for solvents with a high vapor pressure, in order to avoid early evaporation under the given low pressure conditions. Another concept was developed at the INPG in Grenoble. The major difference consists on the generation of discrete droplets rather than a continuous aerosol [45]. This concept has in the meantime been commercialized by JPELEC and is presently distributed by AIXTRON AG under the trademark TRIJET. The advanced systems allows for the simultaneous evaporation from different sources.

At the time, the most advanced commercially available delivery systems, the Liquid Injection Subsystem DLI-25C from MKS Instruments and the LDS-300B from ATMI, use the flash evaporation principle. In order to minimize the impact of the major disadvantage of flash injection systems, precise temperature control of the vaporizer is required to avoid decomposition or clogging due to partial evaporation of the chemical compounds. Accurate distribution of the liquid over the vaporizer surface is also important to assure a reproducible process. 
The system from MKS Instruments is designed for single solutions and uses a stack of up to 99 stainless steel disks as evaporation element. The precursor enters the system in the middle of the hot stack and moves in the radial direction, while the vapor exits at the side of the vaporizer element. The disk stack is pressed together against a spring loaded anvil. The spring constant and the number of disks depends on the vaporization point and the viscosity of the solvent. The construction is designed to compensate the pressure of the liquid supplied by a micro-pump with $\sim 5-10$ bar through the spring force on the disks enabling a constant flow rate [43].

Our reactor is combined with the LDS-300B system from ATMI, which includes a liquid precursor mixing system and a vaporizer unit [46]. The single precursors from up to four tanks are mixed volumetric through magnetic valves. The mixture is then pushed by a twostep piston pump with a pressure of $\sim 50-70$ bar to the vaporizer. The pulsed pressurized precursor liquid hits a hot metal frit where the immediate (flash) evaporation of the liquid takes place. Further details are discussed in Chapter 5.

\subsubsection{CVD reactors}

The reactor is the heart of the CVD system. Basically, it is a sealed vessel where the film deposition takes place. Its design must provide a controlled flow and heat distribution at the desired temperatures and pressures. Due to the extreme environmental conditions in the reactor, e.g. oxidizing conditions and high temperatures, the construction materials must be properly chosen. Typical materials are quartz, stainless steel and coated graphite.

Most of the reactors for oxide deposition operate at low pressures ( 0.1 to 10 mbar) to reduce the number of collisions in the gas phase. The distribution of the precursor vapor is mainly controlled by the inlet system as discussed in 3.1.4. Generally, a laminar gas flow pattern is required to ensure process reproducibility. The flow pattern is a characteristic reactor property, which is controlled by the fixed design of the system and some adjustable process parameters like the total reactor pressure, the susceptor temperature and the individual reactants flow rates. There are two major concepts in the reactor design: the hot wall and the cold wall reactor. Hot wall reactors are isothermal vessels and deposition takes place not only on the substrate but also on the heated walls. In the case of metalorganic precursors, this may lead to premature reactions in the gas phase and on the walls and particle formation. This is the reason why they are generally not used in the deposition of ceramic films.

In the case of cold wall reactors, only the substrate is heated and the walls are kept to a much lower temperature mostly through active water cooling. As a result, maintenance actions, e.g. cleaning, and process drifts due to rector contamination are rather small. A direct consequence of the cold wall principle is that there are large temperature gradients over the susceptor that lead to convection phenomena disturbing the gas flow entering the reactor (see section 3.1.4). This effect complicates the modeling which is necessary to estimate the behavior of a reactor. Infrared lamps are mostly used for susceptor heating but also conductively coupled radiofrequency (RF) resistance elements can be applied for heating. Infrared lamps offer the advantage of heating the wafer from the top. This helps minimizing the previously mentioned temperature gradients.

Fig. 3.14 illustrates the large variety of reactor configurations used to accommodate the many CVD applications. The horizontal and vertical reactors shown in Fig. 3.14a and c/d, respectively, are the most conventional configurations. Both can be used for atmospheric and reduced pressure growth. Low pressure CVD (LPCVD) is the main production technology in the silicon based microelectronics industry for growth of polycrystalline silicon, as well as dielectric and passivation films. 
According to the model presented in Fig. 3.2 there is an inhomogeneous boundary layer over the substrate during deposition. The inhomogeneous precursor diffusion through this barrier is compensated by a slow rotation of the substrate. By tilting the substrate relative to the flow direction, a reduction of the boundary layer thickness can be achieved. Horizontal as well as vertical reactors are commercially available for the growth of 1 " till 6" wafers. Their applicability in the growth of ceramic films has been proven in the case of an AIX-200 reactor from AIXTRON which was successfully used in our institute for the deposition of $\mathrm{BaTiO}_{3}$, $\mathrm{SrTiO}_{3},(\mathrm{~Pb}, \mathrm{Ba}) \mathrm{TiO}_{3}$ and $\mathrm{PbTiO}_{3}$ films [39].

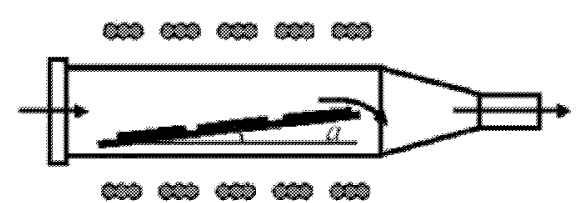

a) horizontal reactor $(a=0)$

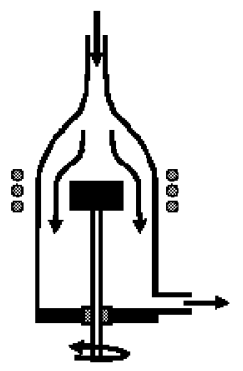

d) vertical reactor

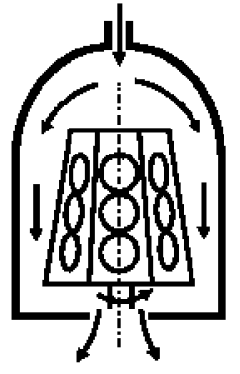

b) bell jar reactor

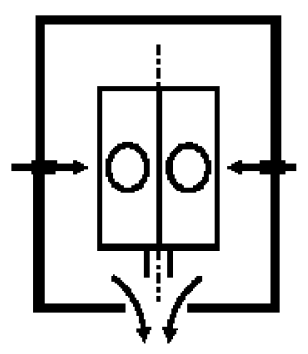

e) barrel reactor

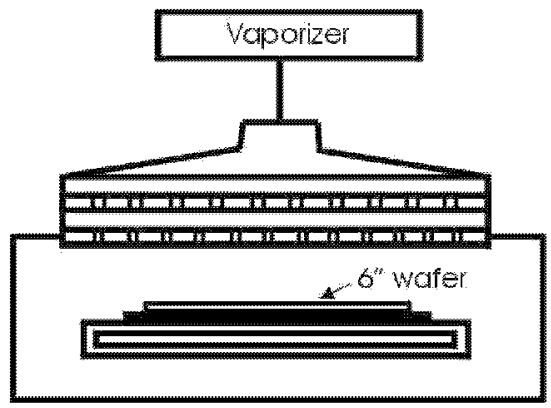

c) Showerhead reactor

Fig. 3.14: Horizontal and vertical reactor designs and their derivatives [43, 47].

The susceptor of a vertical reactor is positioned perpendicular to the gas flow. Problematic in this case is the complex flow structure over the susceptor, which is additionally superimposed by convection driven recirculation phenomena. These effects can be kept to a minimum if the gas inlet is placed closed over the substrate (closed-spaced injection). As an alternative, a showerhead injection system can be used where the gas enters the reactor from many orifices symmetrically positioned in the reactor ceiling [48].

To achieve a high throughput for industrial applications, there have been variations in the basic designs, that allow for batch processing and include the introduction of a further symmetry axis. The barrel reactor (Fig. 3.14e) is derived from the vertical reactor and it is widely used in silicon processing. The horizontal reactor was extended to the bell jar reactor which is being used extensively in the silicon technology (see Fig. 3.14b). A special example is the Planetary Reactor ${ }^{\circledR}$ scheme from AIXTRON [49], which will be described in more detail in the following Chapter. 


\subsubsection{Exhaust system}

The exhaust system treats the effluents, so that hazardous byproducts are disposed in a safe and environmentally sound manner. Mechanical pumps and roots blowers are typically added for low pressure operation. Dry (e.g. charcoal) and wet chemical scrubbers, as well as pyrolytic units, are used to clean up the reactor effluents. Many CVD chemicals require special precautions for safe handling and disposal. Toxic gas monitors are available for a wide range of the chemicals used in the CVD processing. 


\section{Film analysis: structural and electrical properties}

The AIX-2600G3 MOCVD tool used for the deposition of BST thin films is capable of handling up to five $6 "(150 \mathrm{~mm})$ wafers simultaneously and offers the possibility of covering the five satellites of the reactor with different substrates, which are subsequently identically processed. Table 4.1 presents the different substrate types, their size (full wafer sizes are not required for all test experiments), along with the most important characteristics. For example, one satellite carried two 1 " $x 1$ " inch $\left(25,4 \times 25,4 \mathrm{~mm}^{2}\right)$ wafer pieces located in the middle and at the edge of a wafer mask. Three similar pieces were placed on a non-rotating satellite in order to obtain the radial depletion of the precursor incorporation. A third satellite was always loaded with a 6" wafer. The rest capacity of the reactor was covered with dummy substrates, so that growth cannot take place on the satellites which may carry process ready wafers in a later case. On the other side we obtain a process drift depending on the type of the substrate. The reason for this is the different surface temperature of the single substrate types, caused by differences in the temperature emissivity of the top layer. These may also affect the temperature level in the reactor chamber and our processes were calibrated for a full satellite coverage with platinized Si-wafers. Dummy wafers were utilized as masks and the patterns were cut using a laser tool. The masks and the wafer pieces have to be cut precisely in order to prevent gaps, which may cause turbulence in the gas flow.

\begin{tabular}{|c|l|l|l|}
\cline { 2 - 4 } \multicolumn{1}{c|}{} & \multicolumn{1}{c|}{$\mathrm{SiO}_{2} / \mathrm{Si}$} & \multicolumn{1}{c|}{$\mathbf{P t} / \mathrm{SiO}_{\mathbf{2}} / \mathrm{Si}$} & \multicolumn{1}{c|}{$\mathbf{P t} / \mathrm{TiO}_{\mathbf{x}} / \mathrm{SiO}_{2} / \mathbf{S i}$} \\
\hline Use & $\begin{array}{l}\text { XRF, SIMS, early } \\
\text { runs }\end{array}$ & $\begin{array}{l}\text { XRF, electrical } \\
\text { properties }\end{array}$ & Electrical properties \\
\hline Remark & 1 1"x1" & 1 1"x1" & $\varnothing 6 "$ \\
\hline $\begin{array}{l}\text { Located at different } \\
\text { positions for } \\
\text { homogeneity tests } \\
\text { Different surface T } \\
\text { compared to Pt }\end{array}$ & $\begin{array}{l}\text { Poor adherence } \\
\text { Later runs }\end{array}$ & $\begin{array}{l}\text { Supplier: } \\
\text { Availability }\end{array}$ & $\begin{array}{l}\text { Radiant Tech. } \\
\text { AIXACCT } \\
\text { MOCVD-Pt }\end{array}$ \\
\hline
\end{tabular}

Table 4.1: Types of substrates for morphological and electrical characterization.

During the start-up phase as well as in the early process optimization phase, oxidized wafers and 1"xl" Si wafers and pieces were used. Later on, as the grown films reached high quality, additional platinized pieces were included in order to obtain samples with Metal-InsulatorMetal (MIM) structures for electrical characterization. Finally, 6" platinized Si wafers yielded information on the reactor behavior and process characterization. There is a difference in the surface temperature of a small wafer piece compared to a full $150 \mathrm{~mm}$ wafer. This can be attributed to the difference of the thermal coupling between the satellite and the wafer. Generally, the surface of a wafer piece is hotter than that of a 6 " wafer.

The platinized wafers were available in two different layer stacks. The difference is focused on the adherence layer between the $\mathrm{SiO}_{2}$ layer and the Pt layer. This can be realized by $\mathrm{TiO}_{2}$, $\mathrm{Zr}$ or it can be simply missing. The lack of an adherence layer makes it difficult to form a stable $\mathrm{Pt}$ film on the $\mathrm{SiO}_{2}$ layer. Sputtering is widely used for Pt deposition but also MOCVD methods are under development [50]. The presence of a $15 \mathrm{~nm} \mathrm{TiO}_{2}$ film below the Pt 
electrode is a major drawback in case of stoichiometry and thickness characterization by XRF as will be shown below.

All processed wafers were scanned routinely by $\mathrm{x}$-ray fluorescence (XRF) and x-ray diffraction (XRD) in order to determine the film composition and the crystal structure respectively. X-ray methods are widely used for thin film characterization for numerous reasons.

1. Non-destructive: The analyzed samples are not destroyed or changed during exposure to x-rays.

2. Minimal preparation: No pre-treatment necessary. However, samples should be homogeneous without large composition and thickness gradients, because the measurement averages every contribution.

3. Fast: X-ray spectrometry enables quick crystallinity or concentration determination. This is why it is a method of choice for many industries including the semiconductor industry where it can be used for process monitoring.

These methods were complemented in some examples by FTIR analysis for the detection of foreign phases. SIMS and SNMS were used for depth profiling and investigation of the interfaces. In the following sections, 4.2.2 and 4.2.3, these techniques will be described shortly. Particularly XRF was intensively used within the scope of this thesis for stoichiometry and thickness determination. The development and optimization of the method for the application to ceramic thin films is described in detail. Additionally, microscopic methods (AFM, SEM, and TEM) were used to reveal more details of the morphology. In Chapter 4.3 we give an introduction to electrical characterization methods.

\subsection{Film stoichiometry and areal mass density by X-ray fluorescence (XRF)}

In the production of MOCVD thin films we focused on BST with the nominal stoichiometry $\left(\mathrm{Ba}_{0.7} \mathrm{Sr}_{0.3}\right) \mathrm{TiO}_{3}$. Deviations from the nominal stoichiometry of the perovskite can be written in the present case of $\mathrm{BST}$ : $\left(\mathrm{Ba}_{\mathrm{x}} \mathrm{Sr}_{1-\mathrm{x}}\right) \mathrm{Ti}_{\mathrm{y}} \mathrm{O}_{3-\delta}$. The deviations from the stoichiometric composition on the anion sublattice, e.g. by oxygen vacancies, are considered by the number $\delta$. As this number could not be measured with sufficient accuracy it is not considered in the following. The A position in the crystal lattice is occupied by Gr-II atoms and we will use the molar ratio for quantification instead of the number $\mathrm{x}$.

$$
\frac{B a^{m o l}}{S r^{m o l}}=\frac{x}{1-x}
$$

Most of the films where made with a target $\mathrm{Ba} / \mathrm{Sr}$ ratio of $(\mathrm{Ba} / \mathrm{Sr})_{\mathrm{F}}=2.33$. This is equivalent to 70 atoms of $\mathrm{Ba}$ and $30 \mathrm{Sr}$ atoms for every 100 atoms of Ti in the crystal. Where " $\mathrm{F}$ " describes the film stoichiometry in contrast to the stoichiometry in the liquid that is described respectively by the index "L". It was shown that small changes of this parameter do not influence much the permittivity of the thin films and therefore it is not a very critical parameter for the deposition.

In contrast, the Gr-II to $\mathrm{Ti}$ ratio has a much more significant influence on the material properties [107]. We will characterize these deviations by the ratio of the mol masses of the single elements, $1 / \mathrm{y}$.

$$
\frac{(G r-I I)^{m o l}}{T i^{m o l}}=\frac{B a^{m o l}+S r^{m o l}}{T i^{m o l}}=\frac{1}{y}
$$


$\mathrm{y}=1$ characterizes the stoichiometric case, where the number of $\mathrm{Ti}$ atoms in the perovskite crystal matches the number of the group-II atoms (Ba, Sr). Deviations may be due to the presence of antisite atoms, as well as due to vacancies on one of the cation sublattices and finally due to foreign phases (precipitates). A composition of $(\mathrm{Gr}-\mathrm{II} / \mathrm{Ti})_{\mathrm{F}}=1.1$ means a $10 \%$ Gr-II excess and $(\mathrm{Gr}-\mathrm{II} / \mathrm{Ti})_{\mathrm{F}}=0.91$ means a similar Ti excess, respectively. Alternatively, a description based on the $\mathrm{Ti}$ atoms ratio to the total number of atoms can be found in the literature ${ }^{1}$. This ratio is 0.5 for the stoichiometric case, but in this work we will use exclusively the notation described first.

XRF allows to identify and determine the concentrations of the elements present in solids, powders and films. All elements from beryllium (atomic number 4) to uranium (atomic number 92) are measurable in concentrations from $100 \mathrm{wt} \%$ down to a few parts pro million (ppm). Because of the close proximity of the $\mathrm{Ba}$ and $\mathrm{Ti}$ lines we use the wavelengthdispersive XRF spectrometry for the present application. XRF is a standard analytic technique, however, the precision of $0.5 \%$ in the quantitative analysis of the composition required here needed extensive optimization of the measurement process.

\subsubsection{Principle}

The x-ray beam impinges the sample material and high-energy primary x-ray photons hit the atoms in the measured film. As a result, electrons from the inner orbitals are ejected in the form of photoelectrons converting the atoms into unstable ions. The missing electrons are then replaced quickly by electrons from outer orbitals in order to restore a more stable atom state. These transitions are accompanied by an energy emission in the form of a secondary xray photon. This phenomenon is known as "fluorescence". This process is presented schematically in Fig. 4.1.

The different electron orbitals with their corresponding energy levels are called K, L, M, etc., where $\mathrm{K}$ is closest to the nucleus. The energy $(\mathrm{E})$ of the emitted fluorescent photons is determined by the difference in energies between the initial and final orbitals for the individual transitions and can be calculated by the formula:

$$
\begin{array}{ll}
E=h \cdot \frac{c}{\lambda} & \text { Eq. } 4.3
\end{array}
$$

where $h$ is Planck's constant, $c$ is the velocity of light and lambda is the wavelength of the photon. An electron transition from the $\mathrm{L}$ to the $\mathrm{K}$ line is called a $K \alpha$-transition. Similar, $L \beta$ is a transition from the $\mathrm{N}$ to the $\mathrm{L}$ line and so on. This is depicted in Fig. 4.2 for the most common transitions.

After Eq. 4.3, wavelengths are inversely proportional to the energy and characteristic for each element. Hence, it is possible to detect all different elements present in the sample. In addition, the intensity of emission, i.e. the number of emitted photons, given in counts per second (cps), is proportional to the concentration of the responsible element in a sample.

\footnotetext{
${ }^{1}$ Alternative description of the BST composition : $a t \% T i=\frac{T i^{m o l}}{B a^{m o l}+S r^{m o l}+T i^{m o l}}$
} 


\section{Photoelectron}
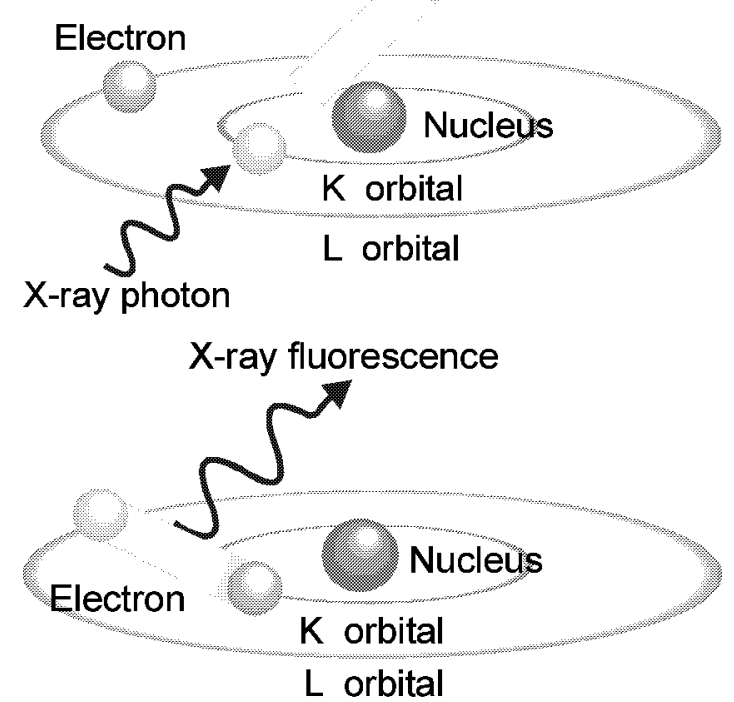

Fig. 4.1a-b: Principle of fluorescence analysis.

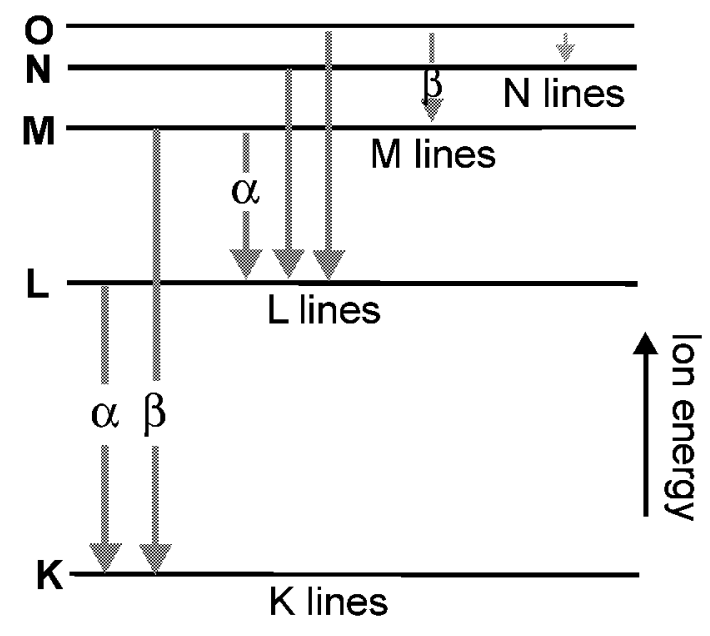

Fig. 4.2: The most common electron transitions after $x$-ray irradiation of an atom.

\subsubsection{Experimental set-up}

The XRF tool is a RIGAKU ZSX-100e with a $3 \mathrm{~kW}$ Rh-tube [52]. The wavelength dispersive element characterization takes advantage of the Bragg law (Eq. 4.12). A well defined analyzing crystal with known distance $d$ between its lattice planes is used to separate the various wavelengths by diffraction, while radiation strikes the crystal at an angle theta, $\theta$. The analyzing crystal and the detector are rotating in the $\theta / 2 \theta$-mode, so that the angle between the x-ray tube and the sample remains constant. A collimator is placed behind the sample to ensure that only photons from a certain angle direction hit the analyzing crystal and these are detected by an appropriate detector, e.g. scintillation counter (SC) or proportional counter (PC). The measurement setup for the XRF analysis is presented in Fig. 4.3.

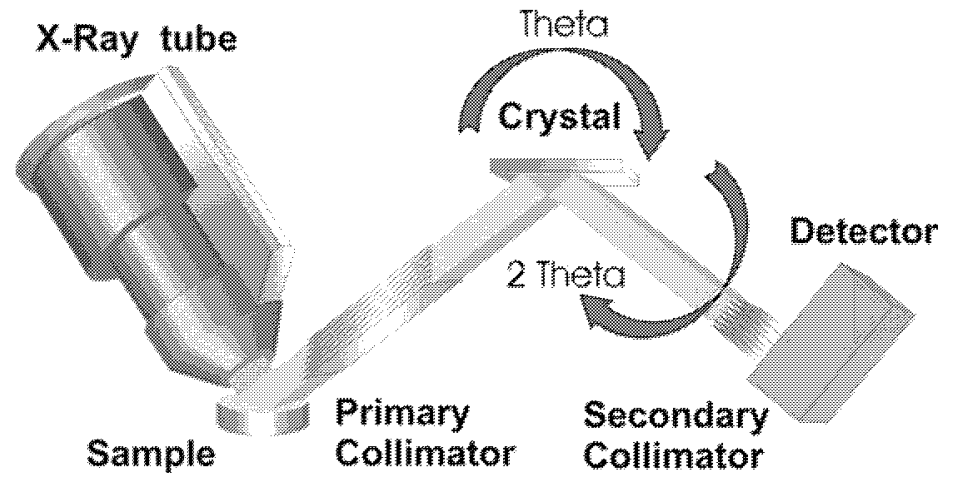

Fig. 4.3: Schematic principle of $X R F$ analysis.

The optimal excitation energy for the desired lines was determined to $50 \mathrm{kV}-60 \mathrm{~mA}$, which is also the maximum tube power. For the analysis of thin films which contain the elements $\mathrm{Ba}$, $\mathrm{Sr}, \mathrm{Ti}, \mathrm{Pb}$ and $\mathrm{Ta}$ the standard analyzing crystal $\mathrm{LiF} 200$ was used with a lattice parameter $2 d=4.0276 \AA$. For $\mathrm{Bi}$, which was overlapped by the Pt L $\beta$ - lines, the $\mathrm{M}$ - line and a Ge crystal with a lattice parameter of $2 d=6.532 \AA$ was chosen. Standard primary and secondary 
collimators were used. A fine secondary collimator leads to a lower background and smaller diffraction effects. On the other hand, the $\mathrm{Sr}-\mathrm{K} \alpha$ intensity decreased down to $40 \%$ of the original value, which was unacceptable for films thinner than $30 \mathrm{~nm}$. The beam diameter was set to $25 \mathrm{~mm}-\varnothing$, the sample size was $1 \times 1$ inch square, and a stainless steel sample holder mask restricted the sample area to a defined size of $A=4,91 \mathrm{~cm}^{2}$ Additionally, the samples were rotated during the measurement in order to average any inhomogeneity in composition and thickness. Two vacuum pumps, one for the sample changer chamber and one for the analysis chamber establish a basic pressure at $3 \mathrm{mbar}$. Vacuum is important in order to avoid secondary collisions of the photoelectrons with air molecules as well as absorption of the Xrays.

\subsubsection{Quantitative analysis}

It is possible to determine all the elements that are present in a thin film by scanning over a wide 2 theta spectrum, e.g. $10^{\circ}-90^{\circ}$. Fig. 4.4 depicts the scan profile of a BST standard, near the interesting lines. The lines are characteristic for the elements in the sample, while the background intensity level depends on the substrate, e.g. platinized wafers have a higher background than $\mathrm{Si} / \mathrm{SiO}_{2}$ wafers.
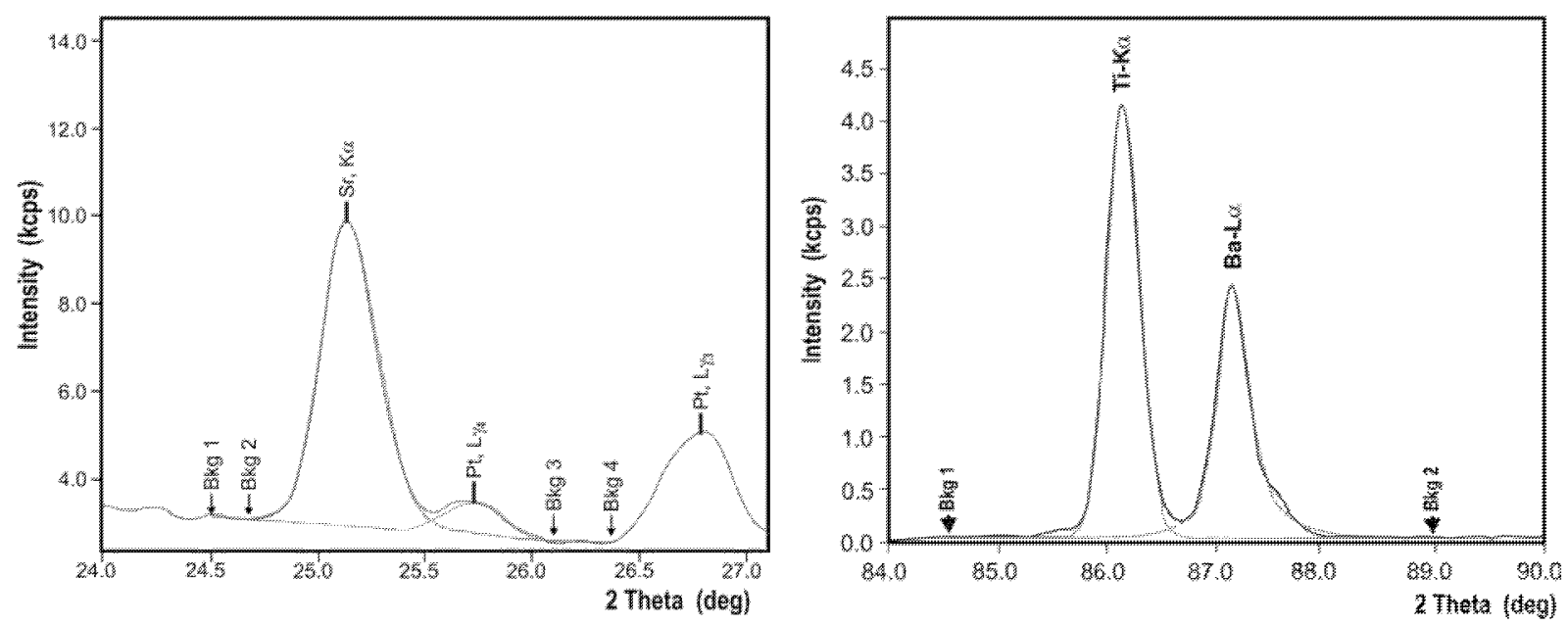

Fig. 4.4: Line spectrum of fluorescence analysis with background fitting and peak deconvolution a) $S r-K \alpha, b) T i-K \alpha$ and $B a-L \alpha$.

For the quantitative evaluation of the integral intensity the peak form can be considered as a pure Gaussian distribution. The intensity calculation starts by fitting the background using a linear, multi-point or polynomial fit. In case of overlapping lines, peak deconvolution is necessary and Gaussian distributions are fitted in the line profile, as shown in Fig. 4.4. Background calculation is different for the high energetic peaks ( $\mathrm{Sr}$ ) than for the low energetic ones at the other end of the spectrum $(\mathrm{Ba}, \mathrm{Ti})$, where the background remains almost constant. In the first case, a 4-point fitting is used to find a mean value for background extraction in the high noise region $( \pm 50 \mathrm{cps})$, in contrast to the 2-point linear fit for the Ti and Ba peaks.

All measuring parameters and conditions were optimized for the investigation of thin films. Since the generation of photoelectrons is a statistical process it is important to optimize the measuring time. XRF is a fast analytical method and a good reproducibility of the peak height below $1 \%$ standard deviation is already achieved after some tenths of seconds depending on the intensity of the appropriate line. For the thin film application the measuring time was 
optimized for both peak and background measurement to ensure good reproducibility and reasonable analysis time. The measuring condition for the quantitative analysis of BST films can be found in Table 4.2.

This table also presents the peak and the background positions for all measured elements. The lines were chosen in terms of their intensity and their stability. In most cases the $K \alpha$-line was utilized for measurement since it represents the highest energetic transition. This is not true in the case of $\mathrm{Ba}$, because its $\mathrm{K} \alpha$ line cannot be sufficiently excited with the given excitation voltage [53]. Therefore, the $\mathrm{Ba}-\mathrm{L}_{\alpha 1}$ line had to be chosen.

\begin{tabular}{cccc}
$\begin{array}{c}\text { Element } \\
\text { line } / \boldsymbol{\lambda}\end{array}$ & Position & $\begin{array}{c}\text { 2 } \boldsymbol{\theta} \text { Angle } \\
\text { (deg) }\end{array}$ & $\begin{array}{c}\text { Detector } \\
\text { time (sec) }\end{array}$ \\
\hline $\mathrm{Sr}-K \alpha$ & Peak & 25.129 & 40 \\
$\lambda=0.877 \AA$ & $\mathrm{BG} 1$ & 24.500 & 20 \\
& $\mathrm{BG} 2$ & 24.700 & 10 \\
& $\mathrm{BG} 3$ & 26.100 & 10 \\
& $\mathrm{BG} 4$ & 26.400 & 20 \\
\hline $\mathrm{Ti}-K \alpha$ & Peak & 86.128 & 40 \\
$\lambda=2.750 \AA$ & $\mathrm{BG} 1$ & 84.500 & 20 \\
& $\mathrm{BG} 2$ & 88.500 & 20 \\
\hline $\mathrm{Ba}-L \alpha$ & Peak & 87.147 & 40 \\
$\lambda=2.775 \AA$ & $\mathrm{BG} 1$ & 84.500 & 20 \\
& $\mathrm{BG} 2$ & 88.500 & 20 \\
\hline
\end{tabular}

Table 4.2: XRF measurement parameters presenting the used lines for BST samples, their position and the optimized measuring time.

Although oxygen can be detected with the present set-up, no quantitative results were deduced. The difficulty of measuring oxygen is twofold. Oxygen has a relative small atomic number (16), and consequently a very long wavelength $(K \alpha .23 .707 \AA)$ which results in strong absorption, low detector efficiency and consequently in long measuring times, in order to achieve the same precision as compared to elements with a higher atomic mass. Moreover, the results might be affected by absorbing layers on the surfaces, interfaces and grain boundaries.

For the quantification of the intensities from deeper layers the absorption must be considered. The penetration depth of the exciting x-rays can be calculated by the Lambert-Beer equation:

$$
\begin{array}{ll}
I_{x}=I_{0} \cdot e^{-(\mu / \rho) \rho x} & \text { Eq. } 4.4
\end{array}
$$

where $I$ is the intensity of the beam just before the sample (position: 0 ) and beyond the thickness $x . \mu / \rho$ is the mass absorption coefficient and $\rho$ the density of the material. The mass absorption coefficient $\mu / \rho\left[\mathrm{cm}^{2} \mathrm{~g}^{-1}\right]$ is independent from the density and has a characteristic value for every element for a certain wavelength. It can be found in tabulated form in [54] and is integrated in the RIGAKU software. The total mass absorption coefficient for the sample can then be calculated by:

$$
(\mu / \rho)_{\Sigma}=\sum_{j=1}^{k}(\mu / \rho)_{j} \cdot C_{j}
$$


$C_{j}$ is the weight ratio of the element $j$ in the sample. Similarly, the fluorescent radiation is absorbed on its way back out of the sample; the wavelength is now that of the corresponding fluorescent line.

In case of our thin films containing elements from $\mathrm{Ti}$ to $\mathrm{Pb}$, the penetration of the $\mathrm{x}$-rays can be several micrometers $(\mu \mathrm{m})$ and is consequently much deeper than the film thickness $(<10 \mathrm{~nm}$ and $150 \mathrm{~nm})$. That means that not only the elements in the desired film but also sublayers and interdiffused elements are taken into account. This can lead to wrong results. For this reason it is not possible to determine the exact stoichiometry in titanate films on substrates with an unknown or inhomogeneous $\mathrm{Ti}$ adhesion layer. Therefore, $\mathrm{SiO}_{2} / \mathrm{Si}$ wafers were used in the beginning and were replaced finally by platinized wafers without an adhesion layer or with a $\mathrm{Zr}$ sublayer. On the other hand, thin samples have the advantage that self-absorption effects in the sample can be neglected and the corresponding 'Fundamental Parameter' correction programs based on the calculation of interelement effects have not been applied.

In addition to the fluorescent radiation, a disturbing background may pass the analyzing crystal of the spectrometer. Of special concern is the diffraction of the primary radiation in the sample. For polychromatic x-rays on polycrystalline thin films these contributions cannot be completely avoided. Additionally, such effects are even less reproducible for films on single crystalline substrates. These effects yield additional peaks (diffraction peaks) in the spectrum, that cannot be appointed to an element. Such peaks can be eliminated, but if they are not revealed from the background, no deconvolution or subtraction is possible. These diffraction effects become more serious with decreasing wavelength and can in principle be avoided by taking a fluorescent wavelength which is longer than the diffraction limit of the sample. In the case of BST, diffraction effects becomes significant for the high energy Sr- $K \alpha$ line. The detection limit for this line was approximated at $\mathrm{m}^{\mathrm{Sr}} \approx 3 \mu \mathrm{g}$ for an irradiated area with a diameter of $2.5 \mathrm{~cm}^{2}$, which is contained in a $8 \mathrm{~nm}$ stoichiometric BST film. Sample rotation may help to eliminate such effects by integrating the diffraction contributions, but its use has to be examined for each film/substrate combination.

A second important background contribution appearing at high energies, corresponding to low theta angles, and comes from the Compton tail of the characteristic $K \alpha$-line of the Rh tube. In order to achieve a reliable background subtraction the background must be investigated for every film substrate combination.

\subsubsection{Absolute calibration}

$\mathrm{XRF}$ analysis is not an absolute method, which means that calibration samples are needed and the evaluation of the results is always relative to the initial calibration. Since the reproducibility of the results, also referred as the precision of the analysis, is very high, the limited factor turns to be the precision of the calibration samples. Their fabrication has to take place using the highest possible manufacturing precision in order to obtain well defined data regarding composition and thickness. The standards were provided by IWE in Aachen and were made using the CSD method. The calibration was performed with a series of four BST standards with thickness between 29 and $106 \mathrm{~nm}$ and the perfect stoichiometry, $(\mathrm{Gr}-\mathrm{II} / \mathrm{Ti})_{\mathrm{F}}=$ 1. In addition, two thickness series with different stoichiometry ranging from Ti-rich, (Gr$\mathrm{II} / \mathrm{Ti})_{\mathrm{F}}=0.94$, to Gr-II-rich, $(\mathrm{Gr}-\mathrm{II} / \mathrm{Ti})_{\mathrm{F}}=1.06$, were supplied in order to test the sensitivity to off-stoichiometry.

The obtained films by CSD methods have exactly the same stoichiometry as determined for the initial solution. However, the absolute mass per $\mathrm{cm}^{2}$ is not determined. Therefore the film 
thickness was measured by DEKTAK with a relative precision of $0.5 \%$ and the mass density for the XRF had to be calculated using the density of the film. This yields a minor error for the stoichiometric films as the bulk density is known (see Table 4.3 below) and as for the CSD films it has been shown to be $99 \%$ of the bulk values. For the off-stoichiometric films some uncertainty in the density may arise.

Using the $(\mathrm{Ba} / \mathrm{Sr})$-composition and thickness of the standards it is possible to calculate the absolute mass ratio of each element in the sample using the density known from monocrystalline $\mathrm{SrTiO}_{3}$ and $\mathrm{BaTiO}_{3}$ samples. For BST the validity of Vegard's Law has been demonstrated and the density can be calculated using the theoretical lattice parameter of a cubic cell, $\mathrm{V}^{1 / 3}$ :

$$
V^{1 / 3}=3.905+0.00104 \cdot x \quad \text { Eq. } 4.6
$$

where $\mathrm{x}$ is the Ba ratio [55].

By varying $\mathrm{x}$ from zero to unity, we obtain the theoretical element density for compositions from $\mathrm{SrTiO}_{3}$ to $\mathrm{BaTiO}_{3}$ using Eq. 4.7:

$$
\rho_{\text {theo }}^{E}=\frac{M^{E}}{N_{A} \cdot\left(V^{1 / 3}\right)^{3}} \quad \text { Eq. } 4.7
$$

$M^{E}$ represents the relative molecular mass of the element $\mathrm{E}$ and $N_{A}=6.02205 \cdot 10^{23} \mathrm{~mol}^{-1}$ is the Avogadro number. The theoretical density values for the most common material systems are numbered in the following table:

\begin{tabular}{c|c|c} 
Material & $\rho_{\text {theo }}^{E}\left[\mathrm{~g} / \mathrm{cm}^{3}\right]$ & Lattice parameter [nm] \\
\hline $\mathrm{BaTiO}_{3}$ & 6.010 & $a=3.996 ; c=4.038$ \\
$\mathrm{Ba}_{0.7} \mathrm{Sr}_{0.3} \mathrm{Ti} \mathrm{O}_{3}$ & 5.758 & $a=3.9778$ \\
$\mathrm{SrTiO}_{3}$ & 5.117 & $a=3.905$
\end{tabular}

Table 4.3: Calculated theoretical densities for some characteristic samples of the BST system [56].

Since the fluorescence intensity is directly proportional to the mass of an element in an unknown sample this mass can be determined from a comparison to the standards:

$$
\begin{array}{ll}
\frac{m^{E}}{A}=c_{s t}^{E} \cdot I_{N}^{E} \quad \text { Eq. } 4.8
\end{array}
$$

$m^{E}$ is the mass of the element in the irradiated surface $A, I_{N}^{E}$ is the net intensity of the examined peak in the XRF spectrum. $c_{s i}^{E}=\left(\left(\mathrm{m}^{\mathrm{E}} / \mathrm{A}\right) / \mathrm{I}^{\mathrm{E}}\right)_{\text {cal }}$ is the calibration factor derived from the standards; in order not to rely on one calibration sample only, the average over the thickness series was obtained as shown in Fig. 4.5.

Fig. 4.5 shows an intensity vs. mass $[\mu \mathrm{g}] /$ illuminated area plot prepared from the data of the calibration standards. The proportionality factor for each element is derived from the slope of the curves. The mass of the single elements in the sample increases proportional to the film thickness as long as the films are homogenous. Additionally, matrix and inter-element effects may be neglected if the film is thin enough, otherwise these effects would have caused a saturation of the curves. The parameters of the linear regression model, $\mathrm{X}=C \cdot \mathrm{I}+D$, that was used for quantification can be extracted from the slope $C$ of the curves after fitting. The representative values for the BST system are as shown in Table 4.4. Intercept D was set to zero. The precision of the samples is also depicted in the quality of the fits, as the correlation factor $R^{2}$ is always near unity. 


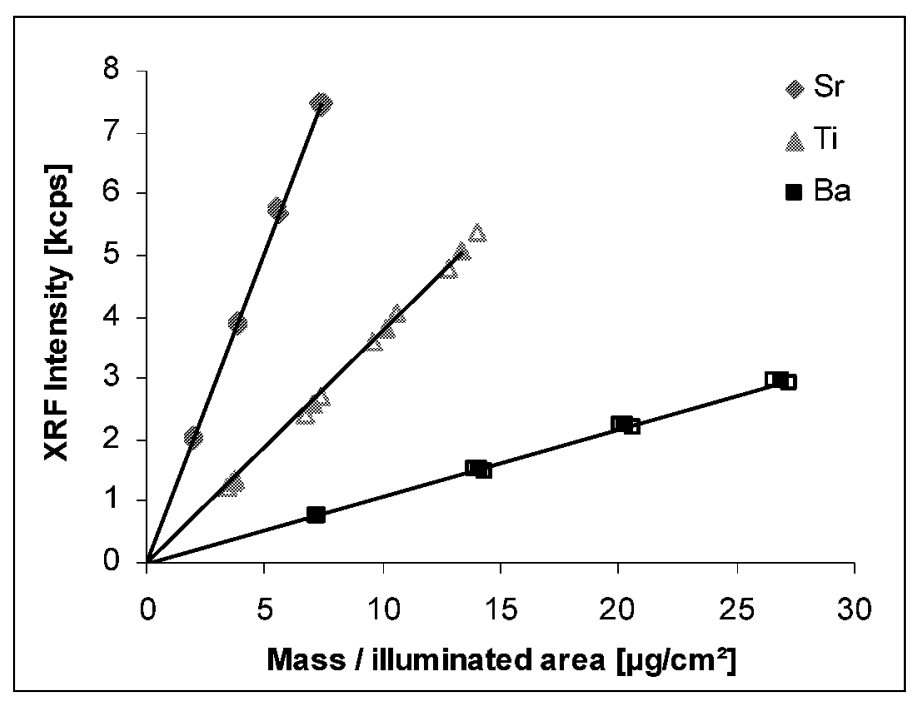

Fig. 4.5: Mass vs. intensity plot for stoichiometry determination of an unknown sample. The graph includes both the stoichiometric (filled symbols) and off-stoichiometric (open symbols) data points.

\begin{tabular}{cccc}
\hline Element & $C$ & $\mathrm{R}^{2}$ & Reproducibility \\
\hline $\mathrm{Sr}$ & 4.84027 & 0.99973 & $0.34 \%$ \\
$\mathrm{Ti}$ & 13.0588 & 0.99966 & $0.21 \%$ \\
$\mathrm{Ba}$ & 44.7132 & 0.99973 & $0.28 \%$ \\
\hline
\end{tabular}

Table 4.4: Extracted parameters from the linear fits in Fig. 4.5.

The measurement precision was extraordinary high and the standard deviation from 10 measurements was $0.35 \%$.

In addition to the stoichiometric points (filled symbols) the off-stoichiometric samples are included (data converted by the same method). As expected, the stoichiometry variation, which is depicted in the scattering of the points, does not effect the average slope of the curves. The solutions for the CSD contained the same concentrations of $\mathrm{Ba}$ and $\mathrm{Sr}$ and the off-stoichiometry was only due to a different content of Ti. Hence, the small differences in the count rates for $\mathrm{Ba}$ and $\mathrm{Sr}$ show the good reproducibility of the CSD method and the changes for Ti indicate the high sensitivity of the method for off-stoichiometry.

From the determined mass densities the widely used stoichiometry ratios, e.g. (Gr-II/Ti $)_{\mathrm{F}}$ or $(\mathrm{Ba} / \mathrm{Sr})_{\mathrm{F}}$, are calculated. Since these values depict the atom or mol ratios, the extracted mass ratios have to be divided by the relative molecular mass of the elements. In the first case we have:

$$
\left(\frac{B a+S r}{T i}\right)_{F}=\frac{\frac{m^{B a}}{M^{B a}}+\frac{m^{S r}}{M^{S r}}}{\frac{m^{T i}}{M^{T i}}}
$$

Finally, we deduce the film thickness, which is proportional to the mass ratio of all elements in the sample, under the assumption of the validity of the theoretical density. Adding the theoretical densities for all elements in the crystal lattice results in the film density: 


$$
\rho_{\text {film }}=\sum \rho_{\text {theo }}^{E}
$$

The thickness of the sample depends on the mass ratio of the elements $m^{E} / A$ in the sample:

$$
t=\frac{\sum \frac{m^{E}}{A}}{\rho_{\text {film }}}
$$

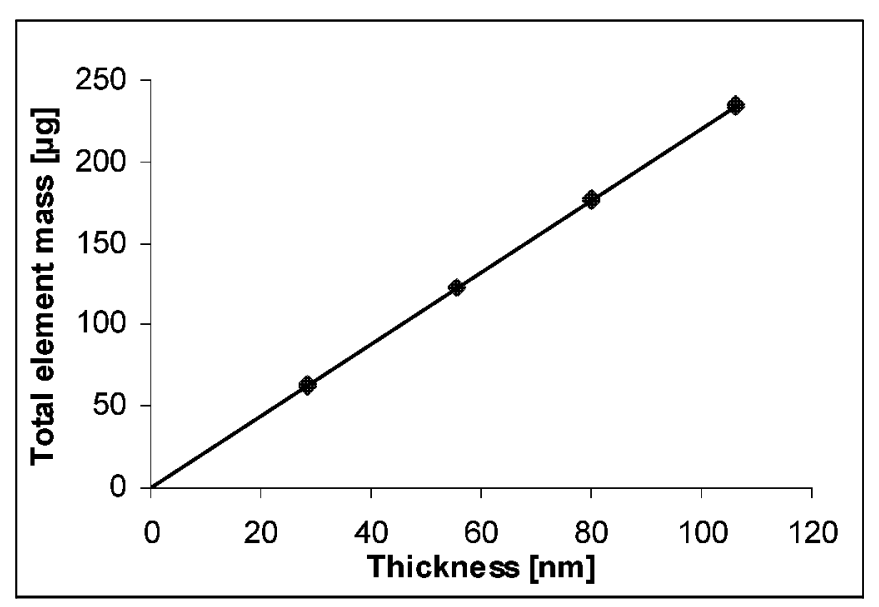

Fig. 4.6: Calculation of the film thickness from the total mass of the detected elements.

Thus, the total mass of the elements in a film is proportional to the film thickness (Fig. 4.6). Stoichiometry variation within a narrow window does not seem to affect the thickness calculation as long as the density is not too far from the ideal perovskite. However, far offstoichiometric, or porous films display large density deviations from the theoretical values, so that the calculated results may contain large errors.

As a final remark, it should be kept in mind, that the given stoichiometry ratios are not affected by the possible deviations of the standards from the theoretical density which affect only the absolute values of the masses /A and the thickness.

\subsection{Structure and morphology}

\subsubsection{X-ray diffraction}

Diffraction of electromagnetic waves or particles (electrons, neutrons, protons) allows the study of the detailed structure of matter if the wavelength is similar to the distance between the atoms or molecules. For a periodic structure the constructive interference of the scattered waves results in sharp diffraction peaks at diffraction angles $(2 \theta)$, as determined by Bragg's law:

$$
2 d \cdot \sin \theta=n \lambda \Rightarrow d=\frac{n \lambda}{2 \sin \theta}
$$

The variable $d$ is the distance between atomic layers in a crystal, and the variable lambda $\lambda$ is the wavelength of the incident X-ray beam; $n$ is an integer. Diffraction only occurs when the distance traveled by the rays reflected from successive planes differs by a number of $n$ wavelengths, where $n$ is an integer. By entering the angular position of the Bragg peaks and the wavelength of the of the x-ray beam in Eq. 4.12, one can calculate the distance $d_{h k 1}$ between successive atomic layers in the crystal. The lattice parameters $a, b$ and $c$ of the unit 
cell and the Miller indices (hkl) can be determined from results from differently oriented grains using Eq. 4.13. For a cubic crystal is $\mathrm{a}=\mathrm{b}=\mathrm{c}$ and for a tetragonal crystal is $a=b$, respectively. Identification of the various crystalline compounds, or "phases", is achieved by comparing the $x$-ray diffraction pattern from an unknown sample with a database of reference patterns such as ICDD (International Center for Diffraction Data) [57].

$$
\frac{1}{d_{h k l}^{2}}=\frac{h^{2}}{a^{2}}+\frac{k^{2}}{b^{2}}+\frac{l^{2}}{c^{2}}
$$

The XRD work was carried out in a Philips X'Pert MRD tool with a copper x-ray tube (characteristic wavelength $\lambda_{C u-\overline{K a}}=1.5418 \AA$ ) and a secondary graphite monochromator [58]. This helps to weaken background radiation effects from the sample, such as fluorescence. Standard powder diffraction scans, so called $\theta-2 \theta$ scans, where the sample is rotated relative to the incident beam by the angle $\theta$, while the angular shift of the detector is $2 \theta$, were preformed in the focusing Bragg-Brentano geometry. Since the monochromator does not exclude the $\lambda / 2$ contributions the samples are usually measured with an offset of $\Delta \theta=2^{\circ}$ in order to suppress the strong (h00) reflections of the single crystalline Si substrate.

In addition the average grain size $d_{k}$ can be determined from the width $\beta(\Theta)$ of the Bragg peaks by using the Scherrer equation:

$$
d_{k}=\frac{K \lambda}{\beta(\theta) \cos \theta} \text { with } K \approx 0.94
$$

Using the symmetrical Bragg-Brentano geometry this value corresponds to the dimension in the direction of the film normal, i.e. for a columnar structure it corresponds to the film thickness.

For some very sharply textured films, rocking curves, i.e. measurements of the angular width of the reflection as a function of the sample rotation $\Theta$ at a fixed Bragg angle $2 \Theta$, were measured in order to characterize the mosaic spread of the nearly single crystalline films.

Measurement of texture (the non-random or preferred orientation of crystallites) involves measurement of the variations in intensity of a single Bragg reflection as the sample is both tilted (psi angle) and rotated (phi angle). The result is plotted as a 'pole figure', in which the contours indicate intensity levels as a function of sample orientation.

Stress is determined by recording the angular shift of a given Bragg reflection as a function of sample tilt (psi). This actually provides a measure of strain distribution in the sample.

$$
\varepsilon=\frac{d-d_{0}}{d_{0}}=\frac{\Delta d}{d_{0}}
$$

Based on the general law of elasticity (Hook's law) which correlates the lattice stain with the applied stress, the actual film stress can be calculated. Under the assumption of a biaxial strain (film strain) and an elastically isotropic film, the general tensor equations can be simplified and yield the so-called $\sin ^{2}$ psi plot [59-61].

$\varepsilon_{\psi}=\frac{v+1}{E}\left(\sigma_{f i l m}-\sigma_{3}\right) \cdot \sin ^{2} \psi+\frac{v}{E}\left(2 \cdot \sigma_{f i l m}-\sigma_{3} / v\right)$

$\mathrm{E}$ and $\mathrm{V}$ represent the elastic modulus and the Poisson ratio, respectively. As the films considered here are bound to the substrate but are free to expand in the direction perpendicular to the surface, there is no stress in this direction, $\sigma_{3}=0$. Hence, Eq. 4.5 is 
further simplified and the film stress is determined from the slope of a plot of the measured $\varepsilon$ against $\sin ^{2} \psi$.

The value of $\Delta d$ at $\psi=0$ corresponds to the lattice parameter perpendicular to the film plane, generally referred to as c-axis, and the extrapolated value of $\Delta d$ at $\sin ^{2} \psi=1$ corresponds to the in-plane lattice parameter. Hence, the tetragonal distortion of the film can be quantified by the total distortion $\mathcal{E}$ or by the c/a-ratio:

$$
\frac{c}{a}=\frac{d_{0}+\Delta d}{d_{0}}
$$

As there is necessarily a change from compression to dilatation there must be a special value of $\psi^{0}$ where there is zero strain, and from Eq. 4.5 we obtain:

$$
\sin ^{2} \psi^{0}=\frac{2 v}{v+1}
$$

Consequently, $d_{0}$, the lattice parameter of the undistorted material, i.e. the value of the corresponding bulk material, can be determined.

Due to the very strong (100) texture of some of the films the standard stress measurement could not be applied, as there is no intensity for a given reflection (hkl) reflection at all tilt angles. Therefore the measurements at different psi angles were performed for different reflection types, e.g. for the (100) textured films:

\begin{tabular}{cc}
\hline Psi measurement & Reflection \\
\hline $\mathrm{d}(\mathrm{psi}=0)$ & $(200)$ and $(400)$ \\
$\mathrm{d}(\mathrm{psi}=36.7)$ & $(321)$ \\
$\mathrm{d}(\mathrm{psi}=45)$ & $(\mathrm{hh} 0)$ \\
$\mathrm{d}(\mathrm{psi}=54.7)$ & $(\mathrm{hhh})$ \\
$\mathrm{d}(\mathrm{psi}=74.5)$ & $(321)$ \\
\hline
\end{tabular}

Table 4.5: Strain measurements for (100) textured films; Reflections and corresponding psi angles.

\subsubsection{Depth profiling by SIMS and SNMS}

Secondary Ion Mass Spectrometry (SIMS) and Secondary Neutral Mass Spectrometry (SNMS) are destructive analysis methods which measure the emitted secondary ions following the bombardment (sputtering) of the sample surface using either noble gas (e.g. argon), or oxygen, cesium and gallium ions with energies up to $25 \mathrm{keV}$ [62]. The used ion energy here was varied between $5-10 \mathrm{keV}$. As can be seen in Fig. 4.7, a primary atom hits the sample surface and penetrates into the matrix up to a certain depth transferring its energy to the sample atoms. Part of this energy returns back to the surface and releases some surface atoms. These can be positive or negative ions, or neutral atoms. The mass spectrometric detection of the sputtered ions is called SIMS and the detection of the post ionized neutrals is abbreviated SNMS. SNMS uses an electron beam to ionize the sputtered neutrals. The post ionization assembly is located some $\mathrm{mm}$ above the irradiated surface and has an entrance aperture of $2 \mathrm{~mm} \emptyset$. Therefore, only the sputtered neutrals emitted in the direction of this solid angle are undergoing the post ionization process. Limited by geometry effects in the 
positioning of the post-ionizer assembly, SNMS is about 3 orders of magnitude less sensitive than SIMS, since the secondary yield is restricted. Common mass spectrometers are quadrupoles or magnetic sector fields. These only allow the transmission of ions with a certain mass to charge ratio to the detector. SIMS is a highly sensitive method with a low sensitivity limit within ppb or below a monolayer, which corresponds to approximately $10^{15}$ atoms $/ \mathrm{cm}^{2}$. Due to the lower yield SNMS may reach a detection range up to $100 \mathrm{ppm}$.

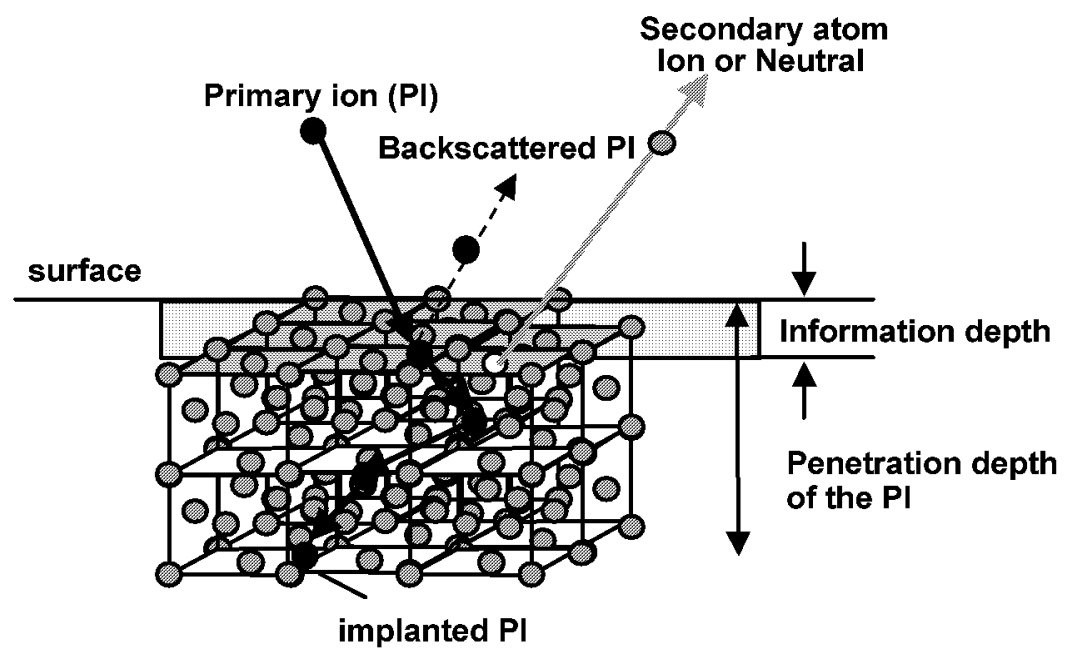

Fig. 4.7: Schematic representation of a surface following an ion bombardment.

Due to large matrix effects, which determine the ionization probability of the emitted ion, SIMS is generally a non quantitative method, except for well defined matrixes like in semiconductors ( $\mathrm{Si}, \mathrm{GaAs}$ ). A well defined matrix also exists in perovskite ceramics like BST, however it is often corroborated by many kinds of defects. Therefore it is sometimes difficult to determine the composition of an interface layer by SIMS. Due to the decoupling of the sputtering and the ionization process in SNMS, matrix effects are negligible. Therefore, it is the method of choice for depth profiling of multilayers. However, a high concentration regime of the major components is required. SNMS is preferably used for investigations of insulating materials, since charging of the surface and its influence on the further processing is avoided. SIMS on the other hand makes it possible to compare different structures, being aware of the possible artifacts, and knowing that the intensity profile may differ from the concentration profile. Sputter rates for SIMS and SNMS are selective depending on the examined material, e.g. Pt is sputtered approx. 2-3 times quicker than BST.

Since XRF analysis averages the signals from a large irradiated area, SNMS could be proven an important complementary tool for measuring the vertical distribution of elements locally in the sample. Especially phenomena close to the interface and interdiffusion of metals into the sublayers can be revealed by this method. However, the examined samples must have a flat surface with a low roughness, in order to achieve high resolution.

\subsubsection{Fourier Transform InfraRed (FTIR) Absorption spectroscopy}

Infrared absorption spectroscopy is a non-destructive technique capable of identifying unknown materials. It is usually applied in the middle IR region for wave numbers from 500 to $4000 \mathrm{~cm}^{-1}$. Some of the infrared radiation is absorbed by the sample and some of it passes through (transmission). The resulting spectrum represents the molecular absorption and transmission, creating a molecular fingerprint of the sample. The peaks in the IR spectrum 
correspond to the frequencies of vibrations between the bonds of the atoms making up the material. Because each different material consists of a unique combination of atoms, no two compounds produce the exact same infrared spectrum. One can then match the spectrum from a measurement with the entries of a database to identify a material or a compound. The size of the peaks in the IR spectrum is a direct indication of the amount of material present and, just like in the XRF analysis, standard materials are necessary for quantification. Our BST films were qualitatively examined by FTIR spectroscopy to monitor the relative contamination through carbonates $\left(\mathrm{BaCO}_{3}, \mathrm{SrCO}_{3}\right)$ during the growth of the films.

FTIR measures all considered infrared frequencies simultaneously. For this an interferometer is used along with a beam splitter. The latter takes the incoming infrared beam and divides it into two optical beams. One beam reflects on a fixed flat mirror. The second beam is reflects on a flat mirror which is moved by a mechanism in a very short distance (typically a few millimeters) away from the beam splitter. The two beams recombine after their reflection at the beam splitter. Because the path that the first beam travels is fixed and the other is constantly changing as its mirror moves, the signal which exits the interferometer is the interference pattern or interferogram of these two beams. This has the unique property that every data point, which is a function of the moving mirror position, has information about every infrared frequency which comes from the source. The measured interferogram signal can not be interpreted directly and decoding of the individual frequencies is accomplished via Fourier transformation [63].

Because there needs to be a relative scale for the absorption intensity, a background spectrum must additionally be measured. This is normally a measurement with no sample in the beam. This technique results in a spectrum which has all of the instrumental characteristics removed. In a next step a platinized wafer without any deposition is measured in order to be used as a reference. By examining a thin film and subtracting all background influences, all spectral features which are still present are strictly attributed to the ceramic layer.

\subsection{Electrical characterization}

After BST growth and structural characterization the electrical properties of the films were determined. Simple MIM test structures, i.e capacitors, were prepared by the deposition of platinum electrodes. Both electrodes consist of $100 \mathrm{~nm}$ sputtered Pt. Wafers from different suppliers were used in order to yield the bottom Pt electrode (see Table 4.1). After MOCVD processing, the $150 \mathrm{~mm}$ wafers are cut by an electrical precision saw into 1 "x1" wafer pieces. A $1-2 \mu \mathrm{m}$ photo resist film is previously deposited on the surface by a spin-on technique, that protects the surface from contamination during processing. The protection coating is then removed from the pieces in an acetone and iso-propanol ultrasonic bath. Nitrogen gas is finally used to clean the surface from any residues. The top electrodes were deposited by magnetron sputtering in sizes between 0.008 and $1.00 \mathrm{~mm}^{2}$ and applying a lift-off process. This is a standard lithography technique where the electrode dimensions are transferred to the photo resist by UV illumination through a chrome mask. After development, contact holes are formed into the resist where the platinum is then deposited. The lift-off step follows the Pt deposition where acetone is used to remove the photo resist that defines the top electrodes. The bottom electrode has then to be revealed at one edge of the sample by etching the BST film away with a $\mathrm{HNO}_{3} / \mathrm{HF} / \mathrm{H}_{2} \mathrm{O}$ solution. This consists of one part $\mathrm{HF}(40 \%)$, one part $\mathrm{HNO}_{3}$ (5\%) and four parts $\mathrm{H}_{2} \mathrm{O}$. Since the lateral dimensions of the Pt/BST/Pt stack are much larger than its thickness, the obtained MIM structures can be considered as ideal capacitors. 
An additional post-annealing step in oxygen ambient was performed ex-situ to recover the films from the damage induced through the sputtering process. Post-annealing is a critical parameter in the integration process as already mentioned in Chapter 1 . The post-processing temperature $\left(550^{\circ} \mathrm{C}\right)$ was always kept below the growth temperature, in order not to influence the crystal and domain structure in the film. The samples were then placed into a pure oxygen atmosphere $\left(\mathrm{O}_{2}\right.$ flow: $\left.200 \mathrm{sccm}\right)$ for $20 \mathrm{~min}$.
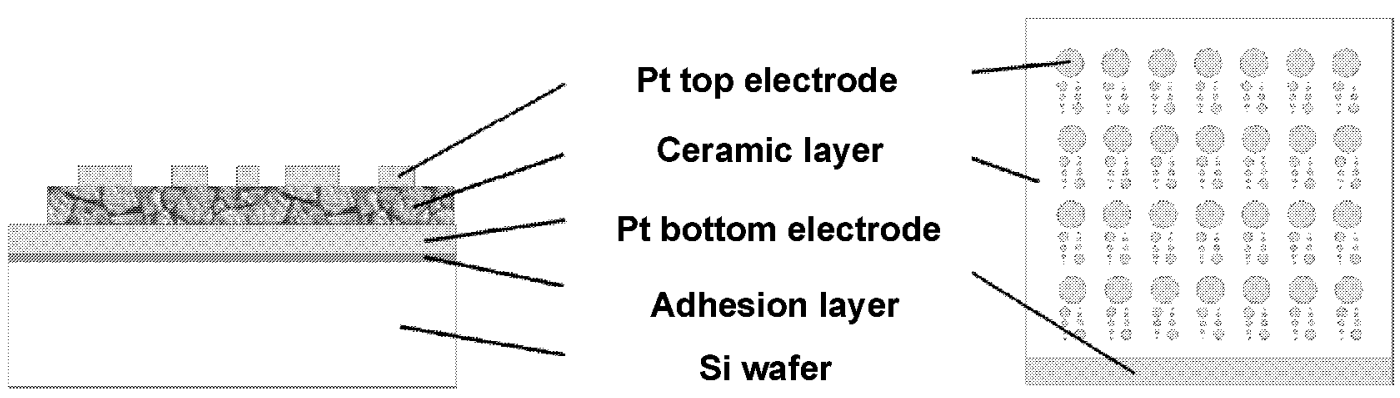

Fig. 4.8: Layer stack on a Si substrate of a sample prepared for electrical characterization.

The standard characterisation included the dielectric permittivity $\varepsilon_{\mathrm{r}}$, which is the capacitors primary feature of interest, measured at a frequency of $1 \mathrm{kHz}$, the dissipation loss and the leakage current. The measurements were performed in a temperature range interesting for DRAM operation from room temperature up to $150^{\circ} \mathrm{C}$.

Extended studies performed on selected samples include DRAM pulse tests, in order to study the self discharge characteristics of the capacitors and short-time measurements to detect the relaxation behavior of electroceramic materials, which is the main issue regarding charge loss in DRAM capacitors. These tests were performed using a modular designed electrical characterization system from aixACCT, the TF ANALYZER 2000 system, with the proper probe heads [64]. The main advantage of the system is that the amplifiers are located in the probe head (module). These can be placed very close to the sample to reduce the incoupling loop between drive and return. The basic unit contains the control unit and the power supplies. An arbitrary waveform generator applies the user defined pulse train to the device under test (DUT). A maximum sample rate of $1 \mathrm{MHz}$ can be achieved and the resolution is 12 bit in the range of $\pm 10 \mathrm{~V}$, that results into $5 \mathrm{mV}$ steps.

\subsubsection{Permittivity and loss tangent}

The permittivity $\varepsilon_{\mathrm{r}}$ and the loss tangent $\tan \delta$ of the thin film capacitors were determined by measuring its capacitance at different bias voltages with the precision LCR meter $H P 4284 A$ from Hewlett Packard [65]. In order to exclude any mutual interference of the measurement signals, especially at high frequencies, a four-terminal pair configuration is employed as shown in Fig. 4.9. The terminals consist of four coaxial connectors and have the advantage of performing both low and high impedance measurements. The $\mathrm{H}_{\mathrm{p}}$ and $\mathrm{L}_{\mathrm{p}}$ lines are current free and cannot cause measurement errors. Regarding $\mathrm{H}_{c}$ and $\mathrm{L}_{\mathrm{c}}$, the same current flows through the central conductor and the outer shield, so that the inductive magnetic fields generated cancel each other thus prohibiting any errors due to self or mutual inductance between the conductors. 


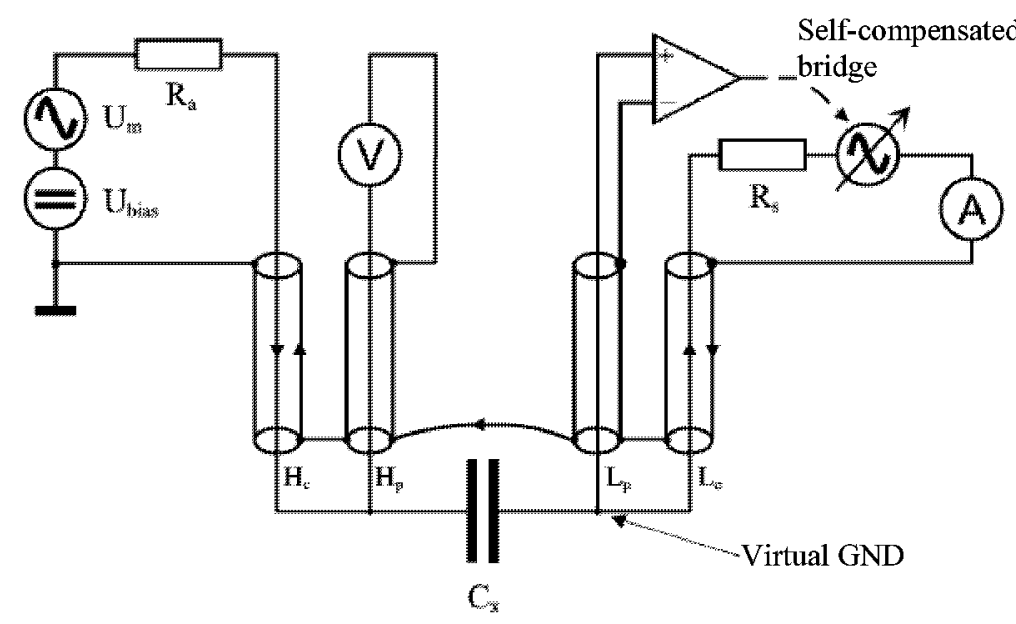

Fig. 4.9: Measurement principle of the HP4284A LCR bridge with four-terminal pair configuration. $C_{x}$ represents the DUT.

The signal level $V_{m}$ across the unknown impedance $Z_{x}$ in a simplified model depends on the oscillator voltage level $V_{o s c}$ and the source resistance $R_{s o}$ of the HP4284A, which is approx. $100 \Omega$ :

$$
\left|V_{m}\right|=\frac{\left|Z_{x}\right|}{\left|R_{s o}+Z_{x}\right|} \times\left|V_{o s c}\right|
$$

Because of the high impedance of the thin film capacitors the signal level is equal to the adjusted oscillator level and an amplitude of $U_{m}=50 \mathrm{mV}$ was found appropriate for most cases. The frequency of the signal can be varied between $20 \mathrm{~Hz}$ and $1 \mathrm{MHz}$. In practice, the standard characterization was performed at $1 \mathrm{KHz}$ in order to avoid high frequency stray fields and low frequency interference with the network voltage. The signal voltage is superimposed with the bias voltage $U_{\text {bias }}$, that generates the desired field across the measured MIM structure.

After contacting a sample a current flows and the voltage drop on the shunt resistance $R s$ is measured and brought to zero after generation of an opposite directed compensation current. From the amplitude difference and the phase shift between the measuring voltage and the compensation current the device calculates the complex admittance or impedance. Through integration of the measured current the capacitance of the sample $C_{p}$ is calculated:

$$
C_{p}=\frac{1}{V_{\text {bias }}+V_{m}} \int I \cdot d t
$$

The index $p$ of the unknown sample capacitance refers to the equivalent electrical circuit of Fig. 4.10 of a capacitor and a resistance connected in parallel. $C_{\mathrm{p}}$ matches the capacitance $C_{\mathrm{x}}$ from Fig. 4.9.

The lateral dimensions of the samples, e.g. round electrodes with $\varnothing 0.1 \mathrm{~mm}-\varnothing 1 \mathrm{~mm}$, are many orders of magnitude larger than their thickness $(10-100 \mathrm{~nm})$ and at intermediate frequencies they can be considered as ideal planar capacitors. The permittivity of the sample is then:

$$
\varepsilon_{r}=\frac{C_{p} \cdot t}{\varepsilon_{0} \cdot A}
$$


$\varepsilon_{0}=8.8542 \cdot 10^{-12} \mathrm{As} / \mathrm{Vm}$ is the dielectric constant of vacuum, $t$ the thickness and $A$ the surface area of the unknown sample. The following equation shows the definition of the dissipation factor ( $\tan \delta$ or $\mathrm{D})$, which is the ratio of the imaginary part of the permittivity to the real part:

$$
\tan \delta=\frac{\operatorname{Im}\left(\varepsilon_{r}\right)}{\operatorname{Re}\left(\varepsilon_{r}\right)}=\frac{X c_{p}}{R_{p}}=\frac{1}{2 \pi f C_{p} R_{p}}
$$

$\mathrm{Xc}_{\mathrm{p}}$ represents the reactance of a circuit including a capacitor and a resistance. In the case of thin films capacitors with small capacitance in the order of $\mathrm{nF}$ or below, the representative circuit can be seen in Fig. 4.10.

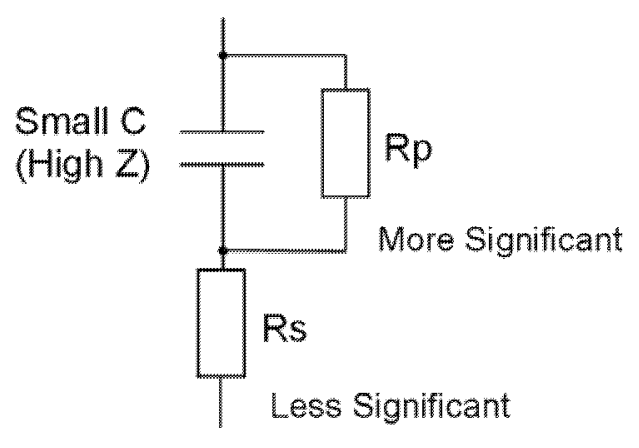

Fig. 4.10: Equivalent electrical circuit representing the measurement components of a thin film capacitor.

The reactance at a given frequency is relatively large and the parallel resistance becomes more significant than the series component. Hence, the complex form of permittivity can be derived from the Eq. 4.23:

$$
\underline{\varepsilon}_{r}=\operatorname{Re}\left(\varepsilon_{r}\right)+j \operatorname{Im}\left(\varepsilon_{r}\right)=\frac{t}{\varepsilon_{0} \cdot A}\left(C_{p}+\frac{1}{2 \pi f \cdot R_{p}}\right)
$$

Not just discrete measurements but also continuous plots can be recorded using the LCR bridge. Two different measurements were performed this way. The sample capacitance was first measured as a function of the bias voltage, the so-called C(V) characteristic. A voltage step method similar to the one described in Section 4.3.3 was utilized, but with much shorter steps, approx. $5 \mathrm{sec}$. Additionally, the frequency dependence of the capacitance was examined in the available range and the results were extrapolated in the $\mathrm{GHz}$ regime, which will be the operation range of the Gbit DRAMs.

\subsubsection{DRAM pulse test}

One of the most important aims in the construction of an integrated DRAM capacitor is its ability to store its load for as long as possible, in order to minimize the need for "refreshing". (see Chapter 1). The DRAM pulse test is a method to determine the self discharge characteristic of our BST capacitors. The operation principle is depicted in Fig. 4.11a and the simulation of the behavior of a storage cell in a memory device is shown in part $b$.

A fast MOSFET switch applies a short, variable voltage pulse to the test capacitor simulating a write cycle with approx. 5ns rise time. The amplitude of the pulse can be adjusted in a large window between $-10 \mathrm{~V}$ and $10 \mathrm{~V}$ and the desirable duration can be set properly between $50 \mathrm{~ns}$ and $650 \mu \mathrm{s}$. After pulsing the switch goes into a high impedance state and the voltage drop 
across the sample capacitor is monitored. Since the MOSFET's off-state leakage would discharge small sample capacitors rather quickly, a mechanical relay is added which opens ca. $1 \mathrm{~ms}$ after the pulse, so that the total system leakage can be reduced to app. 100fA. A high ohmic resistance of the electrometer that traces the self-discharge of the sample is necessary, in order not to discharge the capacitor over the additional measurement path. From the above it is clear that the critical component of the circuit is the fast, high insulating switch.
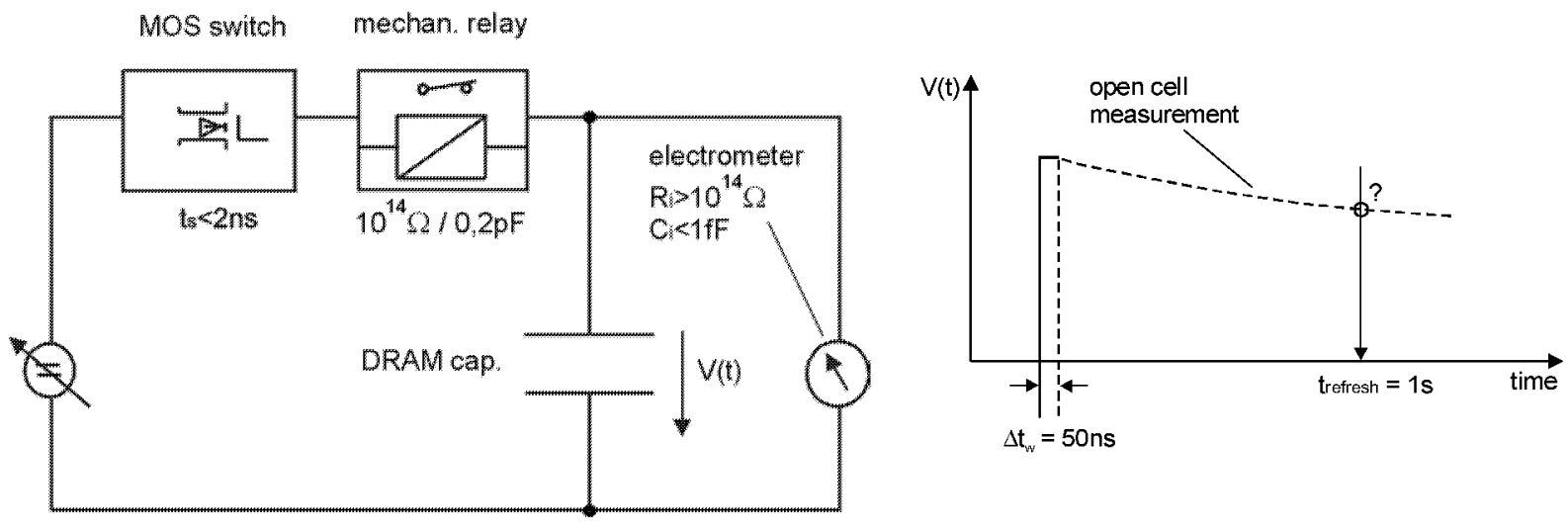

Fig. 4.11: a) Concept of the DRAM pulse measurement device b) schematic pulse simulation plot showing a typical self-discharge characteristic.

Fig. $4.11 \mathrm{~b}$ presents a typical pulse response to a DRAM pulse. The diagram can be generally separated into three sections. The first section depicts the charging of the sample, followed by a voltage drop caused by relaxation phenomena in the sample, that show a $t^{-1}$ behavior known as Curie-von Schweidler law. This is especially true in the case of incomplete charging during write cycles and dominates in the ns to ms range, so that it can only be seen at high sample rates. In the last section, the voltage drop is caused by self-discharge following roughly a $e^{-t}$ behavior. In most cases only this section is visible on the acquired graphs. A maximum of $10 \%$ voltage drop 1 second after the loading pulse in considered to be the lower limit for a DRAM application. Below this value, it is difficult for the sense amplifiers to distinguish between the logic states.

Several sources of measurement errors should be taken into account during the DRAM pulse tests. Incomplete charging of the DUT can result if the pulse is too short or the capacitance too high. Additionally, the resistance of the MOSFET (approx. 30 $\Omega$ ) limits the rise time of the test capacitor's voltage. This should be considered especially when charging with ultra-short pulses. The time to the almost complete charging (99\%) of the capacitor can be estimated by equation:

$$
t[n s]=200 \cdot C[n F]
$$

That means that at least $200 \mathrm{~ns}$ are needed to until a $1 \mathrm{nF}$ capacitor is fully charged. Most of the pulse measurements were performed on the smallest capacitors available $\left(7850 \mu \mathrm{m}^{2}\right)$ in order to minimize size effects caused by larger capacitors, with an average capacitance of $250 \mathrm{pF}$. Using Eq. 4.24 one can see that the capacitors were fully charged, since the minimal pulse length was restricted by the measuring equipment to $50 \mathrm{~ns}$.

The probe connection cable has itself a capacitance of $\approx 100 \mathrm{pF} / \mathrm{m}$ (for the RG58U cable), which can be considered an ideal capacitor in parallel to the sample. This can be a problem when measuring a small sample capacitance, because charge will flow from the cable 
capacitor into the sample, slowing its self-discharge. Our measuring setup includes approximately $0.5 \mathrm{~m}$ of cable $\left(\mathrm{C}_{\text {cable }} \approx 50 \mathrm{pF}\right)$. Consequently, a $50 \mathrm{pF}$ sample will take twice as long to discharge and will show half of the relaxation. A second problem arise from charge injection caused by the MOSFET's gate-drain capacitance during switch off, that partially discharges the sample at the end of the pulse. This may have an effect on very small capacitors $(20 \mathrm{pF})$. Nevertheless, the charging should be traced in the short time region by acquisition with a high sample rate (e.g. $1 \mathrm{MHz}$ ) [64].

\subsubsection{Relaxation current}

If a ceramic capacitor is measured under DC voltage, the time dependent current response of the charging (polarization) and discharging (depolarization) current has the form shown in Fig. 4.12 when plotted in a double logarithmic scale. In the resulting plots three different sections can be distinguished. The relaxation current regime, the leakage current regime, and the degradation or breakdown regime. Relaxation currents control the leakage in the short time region (up to a few seconds) after applying a voltage step. The leakage regime follows the relaxation usually after some tens of seconds and is visible in the flattening of the leakage curves. Finally, electroceramic thin films often display the effect of resistance degradation or breakdown. These effects will be described in the next section.

The relaxation current follows a power law time-dependence known as Curie-von Schweidler behavior and drops exponentially by $t^{-\mathrm{n}}$, where $\mathrm{n} \approx 1$ (see Eq. 4.25).

$$
J(t) \propto t^{-n}
$$

The most common model for the exponential behavior proposed in the literature is a wide distribution of Debye relaxation parts [27]. The relaxation time $\tau$ in the Debye model follows an Arrhenius law:

$$
\tau=\tau_{0} \cdot e^{\frac{W_{0}}{k T}}
$$

Hence, it depends on the thermal energy that is necessary for a dipole to switch from one position to another. The energy for this transition is $W_{0}$. This can be modeled by a single RC element and the distribution of relaxation times leads consequently to a linear superposition of the single relaxation parts displayed in Fig. 4.12. In the inset one can see the resulting equivalent electrical network that causes the exponential characteristic of the relaxation current. It contains an ideal capacitor $C_{\mathrm{HF}}$, a number of $R_{\mathrm{k}} C_{\mathrm{k}}$ elements (instead of a resistance $R$, a conductance $G$ is used here) and a conductance element $\mathrm{G}_{0}$ that represents the leakage current. The model also includes the field dependence of the leakage current, that was found in the examined samples (see Chapter 6.2).

The current measurements in the short time regime are performed by the relaxation current amplifier from aixACCT. This module uses a logarithmic and a linear current amplifier, that allow tracking the current over several decades. The variable linear amplifier was used for the measurements below the microsecond range covering just two decades because the log amplifier is not fast enough. The operation principle is presented in Fig. 4.13. During the application of a chosen voltage step the polarization current is measured, followed by a discharging (depolarization) measurement by switching the voltage back to zero. Since a logarithmic amplifier can only handle a single current direction, two different circuits are implemented for charge and discharge currents. 


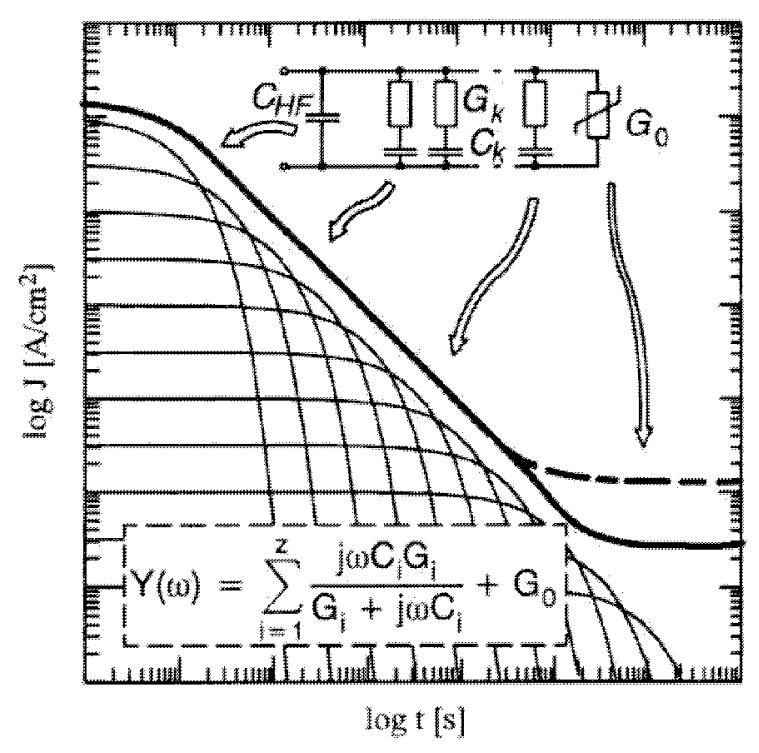

Fig. 4.12: Equivalent network and its development describing the relaxation behavior of dielectric thin films.
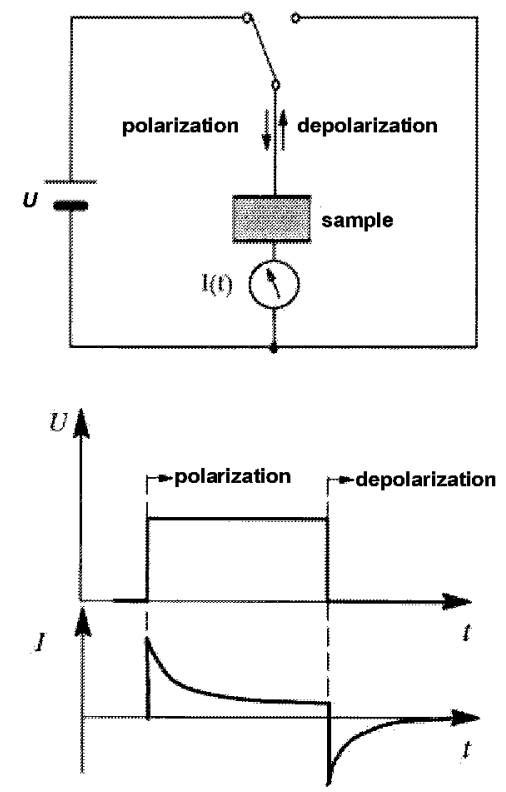

Fig. 4.13a-b: Measurement principle for short time currents. The polarization is followed by a depolarization step.

The relaxation current is being traced in the time regime from $1 \mu$ s to $10 \mathrm{sec}$ for several orders of magnitude from $10 \mathrm{~mA}$ to $10 \mathrm{pA}$. While measuring the relaxation current, one must be aware of the possible error sources. The short time regime is affected by the shunt serial resistance, showing an exponential decay with a time constant equal to the sample capacitance multiplied by the shunt resistance. For the typical capacitor of $1 \mathrm{nF}$ the time constant is $\sim 30 \mathrm{~ns}$. The amplifier itself can also show a relaxation like behavior. This effect can be determined by measuring a reference capacitor with very low relaxation (e.g. air, polystyrene, mica). The amplifiers induced relaxation part can then be subtracted from the measurements on the BST capacitors [64].

\subsubsection{Leakage current}

The leakage current regime follows the relaxation time regime and is recognizable as a plateau in the $\log J$ - $\log t$ plot (Fig. 4.14). This extends in the range from a few seconds up to a time where resistance degradation is initiated. The duration of the leakage regime and consequently the beginning of the degradation behavior cannot be exactly predicted and it depends on external conditions, e.g. applied field or temperature. This is expressed in the rise of the leakage current under DC bias and is often followed by a dielectric breakdown. The lifetime $\tau$ of the sample can be roughly approximated through a thermal activated process:

$$
\tau \propto \exp \left(\frac{W_{\mathrm{deg}}}{k_{B} \cdot T}\right)
$$

$W_{\text {deg }}$ is here the activation energy, $k_{\mathrm{B}}$ the Bolzmann constant and $T$ the temperature. The lifetime of a sample not only depends on the temperature but also on the applied field and the electrode material. Zafer [67] describes the current in the degradation regime in case of Schottky emission with: 


$$
\begin{array}{ll}
J(t)=J(0) \cdot e^{\frac{\Delta W(t)}{k T}}, \text { where } & \text { Eq. } 4.28 \\
\Delta W(t)=W_{\max }\left(1-e^{-\beta \cdot \frac{t}{\tau}}\right) & \text { Eq. } 4.29
\end{array}
$$

is the lowering of the barrier height. The extrapolation for the lifetime of a $40 \mathrm{~nm}$ BST capacitor and $1 \mathrm{~V} \mathrm{DC}$ bias at an operation temperature of $125^{\circ} \mathrm{C}$ was 16 years.

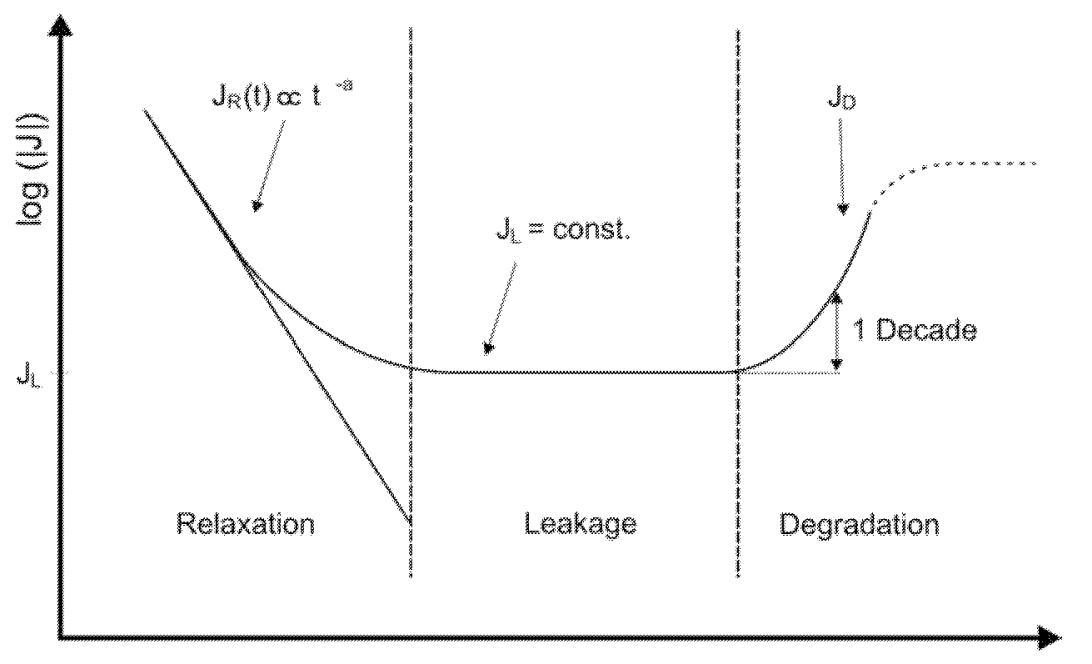

Fig. 4.14: Current function of a ceramic capacitor displaying typical "bathtub" behavior during DC load. The dotted line in the degradation characteristic depicts a possible trend suggested by [67].

$\log ($ time)

The leakage current investigations were performed at several probe stations at different sites (IFF / FZ Jülich and IWE-II / RWTH Aachen). The measurements at these probe stations are capable of covering a large time and temperature region. The main setup that was implemented at our site in the IFF consists of the noise poor voltage generator Burster 4462 and a Keithley 617 electrometer, which are connected to the sample as shown in Fig. 4.15, so that the positive voltage is applied at the top electrode [PoT]. A control unit (computer), addresses the voltage generator to set the appropriate voltage level at the DUT and the current values are then acquired by the electrometer. The voltage generator is capable of adjusting any voltage level in the region $\pm 30 \mathrm{~V}$ with a resolution of $0.1 \mathrm{mV}$ [68]. The Keithley electrometer has a minimal detection range around 50fA, but this value varies strongly due to many possible external influences on the measurement [69]. The measurement method is considered to be the most important issue in a measuring system and it is important to choose a measuring instrument that adds only a minor amount of error to the signal from the DUT. Thus, noise, drift and other sources of error that will be described in the following must be held at a minimum in order to reach the desired accuracy.

Electrometers are DC multimeters with very high input resistance up to $10^{16} \mathrm{ohms}$ and extremely low input current down to $10^{-17}$ amperes. Due to these abilities electrometers are far superior compared to digital multi-meters. In our case, it is desirable to record the leakage current over many orders of magnitude from $10^{-13} \mathrm{~A}$ to $10^{-3} \mathrm{~A}$ in order to determine the suitability of a BST capacitor. A leakage current below $10^{-8} \mathrm{~A} / \mathrm{cm}^{2}$ at $1 \mathrm{~V}$ bias is still considered suitable for the application of the films as dielectric material in the DRAM capacitors of the $1 \mathrm{Gbit}$ generation. The influence of the measuring device on the test circuit has to be determined, so that the original current flowing through the DUT does not differ from the current indicated on the ammeter. 


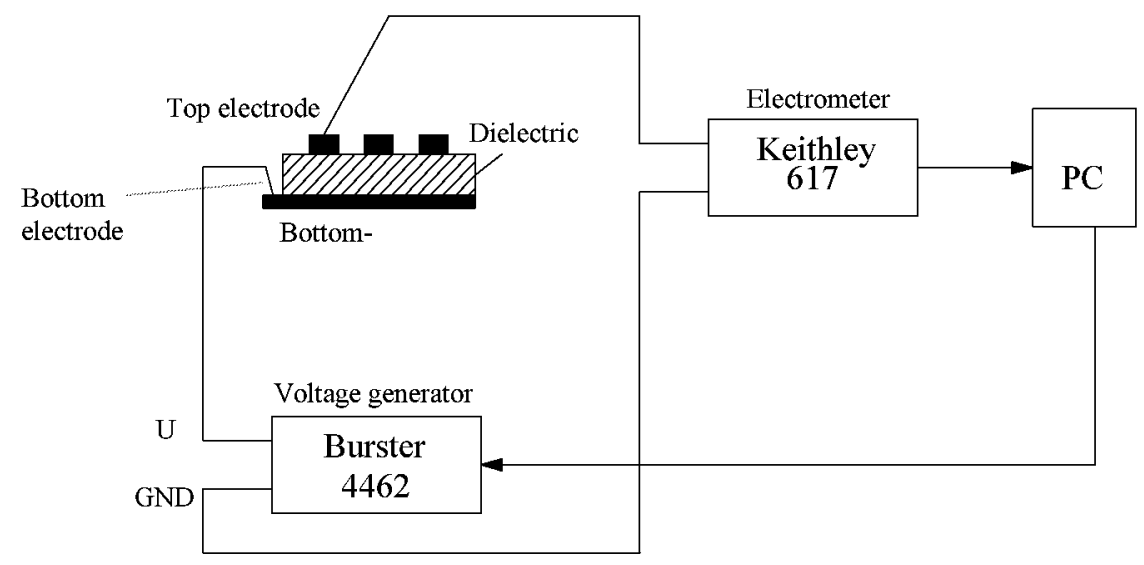

Fig. 4.15: Measurement setup for leakage current investigation.

The ammeter can be modeled as shown in Fig. 4.16, consisting of three separate circuit elements [69]. The shunt resistance $\mathrm{R}_{\mathrm{SH}}$ is caused by the cable that is connected to the meter. The generator $\mathrm{I}_{\mathrm{E}}$ represents the unwanted contributions to the total current coming from the cabling and the connectors. Finally, the internal electrometer resistance $R_{M}$ is in series connected with an ideal ammeter $\mathrm{M}_{\mathrm{I}}$.

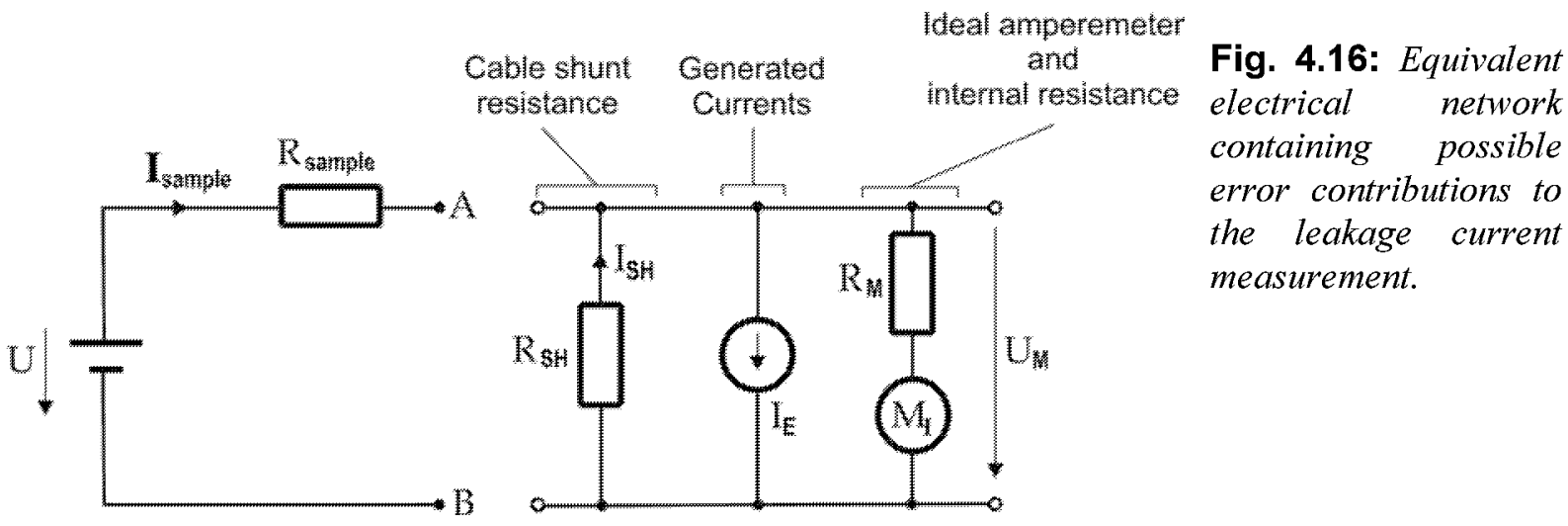

By connecting an ammeter in a circuit (voltage generator: $V$; source resistance: $R_{S}$ ), the current indicated on the meter is equal to the current that would flow in the original circuit, plus the errors coming from the elements in the model. This current can be calculated using the formula:

$$
I_{M}=\frac{V}{R_{S}}-\frac{V_{M}}{R_{S}}-I_{S H}-I_{E} \pm U_{M}
$$

These errors consist of the parasitic currents generated by the elements in the model mentioned above and the inherent meter uncertainty $\mathrm{U}_{\mathrm{M}}$.

All modern electrometers are designed based on the feedback ammeter principle. This is depicted in Fig. 4.17, that shows the small signal equivalent circuit of the feedback method. This is basically a current controlled voltage generator. The input and output voltages of the amplifier are given by Eq. 4.31 and Eq. 4.32, respectively:

$$
U_{I N}=I_{I N} \cdot R_{I N}
$$




$$
U_{\text {OUT }}=-I_{I N} \cdot R_{F}-I_{O U T} \cdot R_{O U T}
$$

$I_{\mathrm{IN}}$ flows through the feedback resistor $R_{\mathrm{F}}$. The low offset current of the amplifier can be neglected in the current $I_{\mathrm{IN}}$. Additionally, $R_{\mathrm{IN}}=0$ in case of an ideal amplifier. From Eq. 4.31 it follows that $V_{\mathrm{IN}}=0$. Finally, it is $R_{\mathrm{OUT}}=0$, so that $V_{\mathrm{OUT}}=-I_{\mathrm{IN}} \cdot R_{\mathrm{F}}$. Thus, the output voltage is a measure of the input current. The sensitivity of the method can be controlled by the feedback resistor $R_{\mathrm{F}}$. This is adjusted, so that the output voltage is $>10^{-6} \mathrm{~V}$, so that it can be measured by a voltmeter. On the other hand, the extremely small input resistance allows rapid rise times of the output voltage.
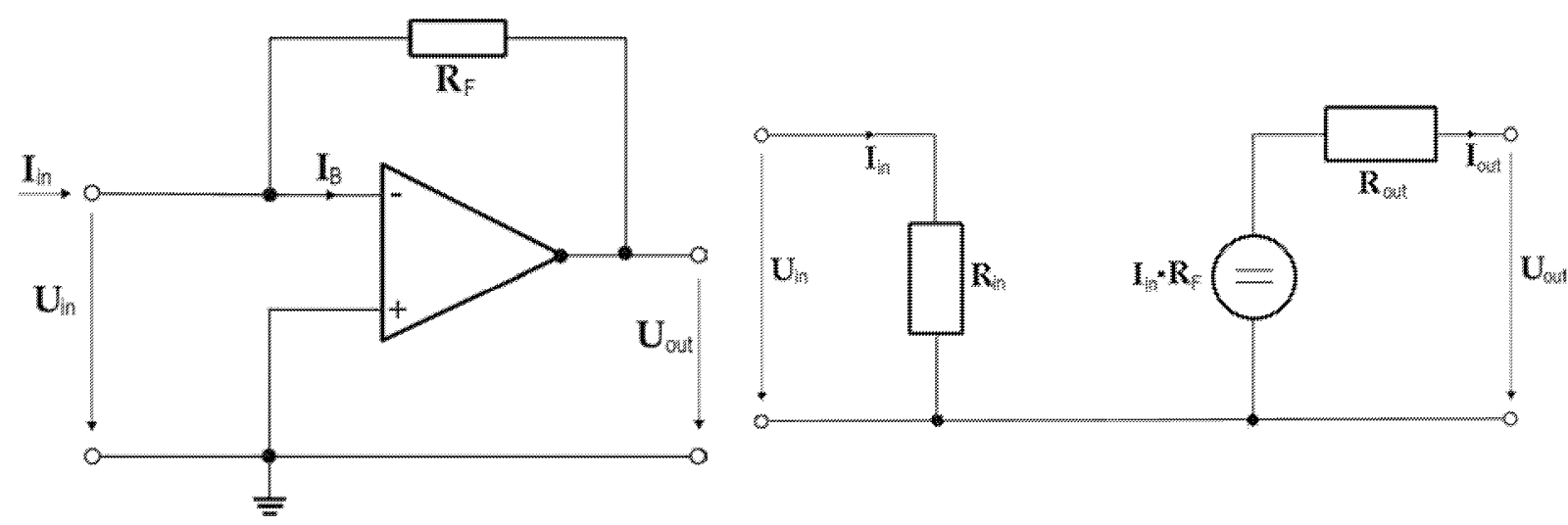

Fig. 4.17 a) Principle of the feedback ammeter b)Small signal equivalent circuit of the the feedback ammeter.

Among the different electrical characterizations, the measurement of the leakage current is the most critical due to numerous parasitic influences that must be taken into account: noise currents may arise from triboelectric, piezoelectric, or electrochemical effects. These are summarized in the term $I_{\mathrm{E}}$ of Eq. 4.30. Triboelectric currents are generated due to friction at the interface between conductors and isolators in cables. Low noise coaxial cables can help to suppress such currents in the region below $<10^{-12} \mathrm{~A}$. Piezoelectric currents are generated in certain isolators, ceramic or crystalline materials after application of mechanical stress. These currents are often in the range of $10^{-13}-10^{-15} \mathrm{~A}$. Noise is also generated by a thermal activated resistive leakage current, i.e. Johnson noise. This current is given by:

$$
I=\sqrt{\frac{2 \cdot k_{B} T \Delta f}{R}}
$$

where $\mathrm{k}_{\mathrm{B}}$ the Boltzmann's constant, $T$ the temperature in $\mathrm{K}, \Delta f$ noise bandwidth and $R$ the resistance value.

Other considerations and precautions before or during the measurement include a sufficient warm up time of the instruments $(<2 h)$ to obtain the rated accuracy. All cables should be fixed firmly to avoid cable flexing which could lead to triboelectric effects. Even with low-noise cabling, noise currents in the order of tens of femtoamps can be generated by cable movement.

While determining the measurement setup it is important to prevent any ground loops. Such loops in multiple instrument setups may create error signals due to induced voltages because of magnetic flux through the loop. Therefore, the instruments should be grounded at a single point. Electrostatic interference is also a usual source of error and occurs when an electrically 
charged object is brought near the sample. This is often the case when hand or body movements near the experimental setup cause fluctuations in the reading of the instrument. Power lines and other sources should be kept away from the experiment and shielding of the sample should be ensured, in order to minimize electrostatic effects. Shielding also helps suppressing the radio frequency interference, which can be a problem especially at low signal levels. Such effects are often expressed as an unusually large offset current or sudden variations in the reading, in the case of impulse sources.

Because of the above it is important to use only triaxial cable for signal connections and make sure that the DUT and the connectors are free of contamination to prevent any unwanted current paths. Since any circuit itself has an inherent capacitance $C_{\mathrm{IN}}$ due to the cabling or instruments, this could affect the response time of the measurements, so generally at least five time constants $(5 \tau)$ should be waited to assure an accuracy better than $1 \%$ :

$$
\tau=R_{S} \cdot C_{I N},
$$

where $R_{\mathrm{S}}$ the source resistance. The influence of the effect can be kept under control by using as short as possible low capacitance cabling.

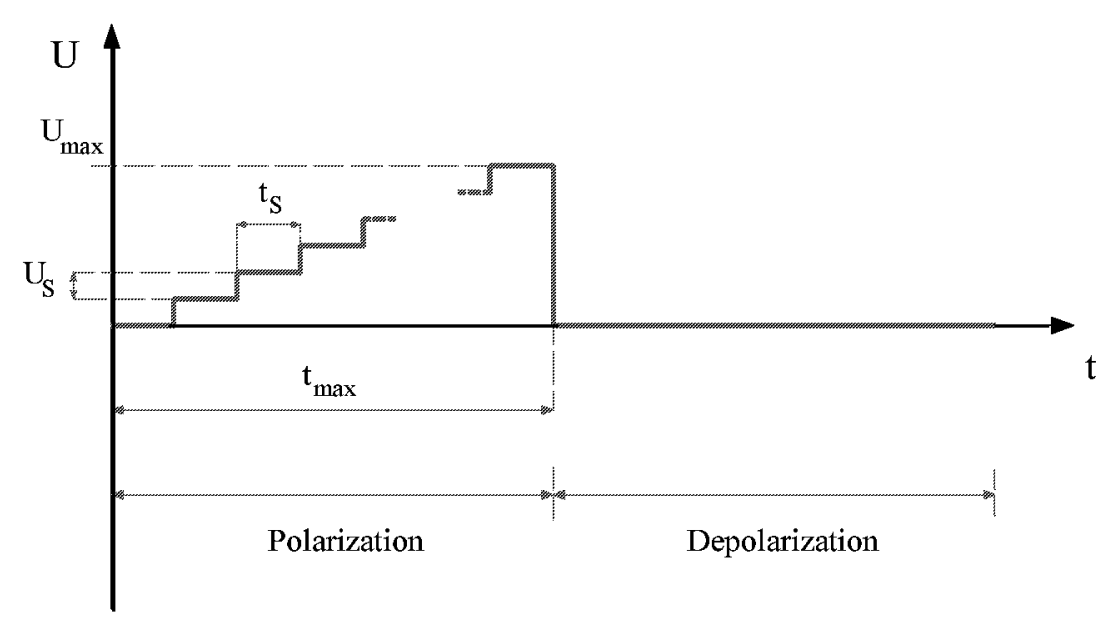

Fig. 4.18: Voltage stair for leakage current investigations. By shorting the capacitor a depolarization step is performed.

After relaxation, the current enters into a regime where leakage dominates. Leakage is often presented in a graph, where the current density is plotted versus the electrical field. A voltage step method, as shown in Fig. 4.18, is widely used for leakage current investigation, but also other methods, including depolarization between steps, are possible [70].

The appropriate voltage function for this measurement is:

$$
V(t)=V_{S} \cdot \operatorname{int}\left(\frac{t}{t_{B}}\right) \cdot \operatorname{sgn}\left(\operatorname{sgn}\left(t_{\max }-t\right)+1\right),
$$

where $V_{\mathrm{S}}$ is the height of a voltage step, $t_{\mathrm{S}}$ the length of a time step and $t_{\max }$ the total (de-) polarization time. The int and sgn functions in Eq. 4.35 are defined as follows [71]:

$$
\operatorname{int}\left(\frac{t}{t_{S}}\right)=n, \text { where } n \cdot t_{S} \leq t \leq(n+1) \cdot t_{S} \text { and } n=0,1,2, \ldots
$$




$$
\begin{array}{rll}
1, & \text { if } & t>0 \\
\operatorname{sgn}(t)=0, & \text { if } & t=0 \\
-1, & \text { if } & t<0
\end{array}
$$

After each voltage step, current acquisition happens once every second for the desired time interval $t_{\mathrm{S}}$, until the relaxation effects have no influence on the total current. This is normally the point where the current reaches its minimum value. The leakage regime of the BST thin films is not a flat plateau, but displays a slight decay over time. The minimum current value at the end of the acquisition time for a certain voltage step is then defined as the leakage current. This is the case after a sufficient large time has elapsed, usually a few tenths of seconds. The end of the leakage current regime was not obtained from room temperature measurements and longer time steps than 1000 s are needed. The number of steps and the step height define the maximum applied voltage, which is set appropriate in order not to reach the breakdown limits. After polarization, a depolarization step follows, where the voltage is set back to zero and the current is recorded over several minutes, so it can be seen whether there is any drift in the initial current level due to irreversible mechanisms. The typical values for the leakage current measurement are presented in the next table:

\begin{tabular}{rl}
\hline Parameter & Value \\
\hline $\mathrm{V}_{\mathrm{S}}$ & $0.05-0.1 \mathrm{~V}$ \\
$\mathrm{~V}_{\max }$ & $2 \mathrm{~V}-8 \mathrm{~V}$ \\
$\mathrm{t}_{\mathrm{S}}$ & $100-1000 \mathrm{~s}$ \\
$\mathrm{t}_{\max }$ & $3000-30000 \mathrm{~s}$ \\
$\mathrm{t}_{\text {depol }}$ & $500 \mathrm{~s}$ \\
\hline
\end{tabular}

Table 4.6: Typical parameters of the voltage step method for leakage current measurement.

In order to compare the leakage currents of films with different thickness the voltage values were adjusted accordingly. Consequently, the maximum value of the voltage stair varied depending on the thickness of the films, e.g. the electrical field after applying an $8 \mathrm{~V}$ bias on a $120 \mathrm{~nm}$ film corresponds to a $2 \mathrm{~V}$ bias voltage applied on a $30 \mathrm{~nm}$ sample $(667 \mathrm{kV} / \mathrm{cm})$. 


\section{The AIX-2600G3 MOCVD tool and its modification for oxides}

\subsection{System description}

Most of the experimental reactors currently used for the development of mass production tools are conventional single wafer showerhead designs [74-76]. In contrast to the current trend towards single substrate reactors, which can be integrated with cluster tools along with other manufacturing process steps, AIXTRON focused on the Planetary Reactor ${ }^{\circledR}$ principle initially patented from Philips [77]. Planetary systems are capable of offering extremely high throughput due to possible batch mode processing resulting in low cost of ownership. The present reactor in our clean room facility is a prototype derived from the III-V multicomponent semiconductor deposition tool. This reactor type was already well established in this narrow market segment because it offers good homogeneity, high efficiency of the precursors and last but not least mass-scale production on large substrates. Furthermore, there is an option for a load lock system which is necessary for mass production. For the deposition of oxide films like $(\mathrm{Ba}, \mathrm{Sr}) \mathrm{TiO}_{3}$ the reactor was combined with a liquid delivery system from ATMI (LDS-300B).

The liquid delivery system consists of a precursor mixing system and the vaporizer unit. The operation principle is presented schematically in Fig. 5.1. The single precursors from up to four tanks are mixed volumetric through magnetic valves. The mixture is then pushed through a capillary tube (1/16" liquid line) by a two-step piston pump to the vaporizer with a pressure of $\sim 50-70$ bar. The vaporizer system includes two exits to the reactor and the ventilation that are switched by pneumatic valves.

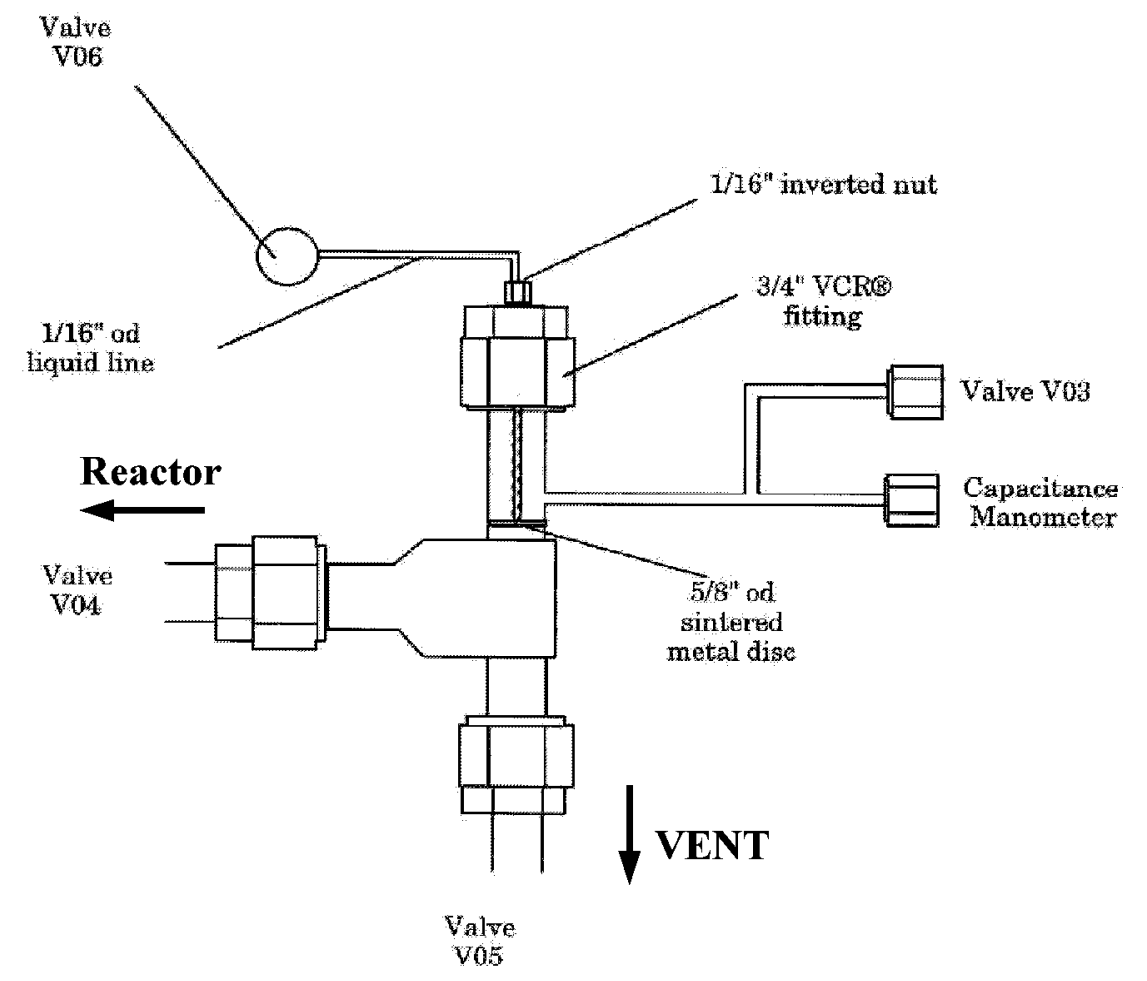

Fig. 5.1: Evaporation zone of the $L D S-300 B$ system. Contact evaporation occurs as the precursors hit on the hot frit (sintered metal disc) [78].

The vaporizer is placed in the immediate vicinity of the reactor. It consists of the vaporizer element, which is a metal frit, and heat elements holding the frit at the appropriate temperature. This temperature is determined by the evaporation temperature of the least volatile component of the precursor mixture. In combination with a check valve (V06) the 
feed pump yields a pulsed high pressure precursor liquid which hits a hot metal frit where the immediate (flash) evaporation of the liquid takes place. Hot carrier gar (Ar) entering through V03 transports the vapor to the reactor. A $10 \mathrm{~min}$ run through the ventilation line (VENTV05) is always performed before the regular deposition process to establish stable evaporation conditions. During this time valve V04 remains closed and the vapor flows through V05 to a cold trap. After this time has elapsed, the valve status is alternated, e.g. V05 closes and the vapor enters the reactor through the open V04 valve. The line to the reactor was kept as short as possible and held at the evaporation temperature in order to avoid condensation of the precursors which results into particle generation and subsequent yield loss.

The temperature control of the frit itself is also of great importance. The evaporation of the precursors cools off the surface allowing the formation of precursor residues which may clog the vaporizer. For that reason, the sintered metal frit has to display great heat capacity to minimize temperature oscillations on the surface. Since it is not possible to avoid the residues due to incomplete evaporation or decomposition, a cleaning step using pure solvent (butyl acetate) is performed after each deposition. In spite of the cleaning steps, clogging cannot be completely avoided. The manufacturer's specification of the vaporizer element suggests its replacement after 200 runs. This number could not be reproduced by far in our investigations and a maximum of 80 runs was performed before partial or total clogging of the vaporizer [78].

The thin films discussed in this work are grown out of a liquid mixture containing precursors from three different sources: 0.35 molar solutions of $\mathrm{Ba}(\text { thd })_{2}$ and $\mathrm{Sr}(\mathrm{thd})_{2}$ and a 0.4 molar solution of Ti(O-i-Pr $)_{2}(\text { thd })_{2}$ dissolved into butyl acetate. Different stabilizers had been added, tetraglyme in the beginning and later on amide based adducts manufactured by ATMI.

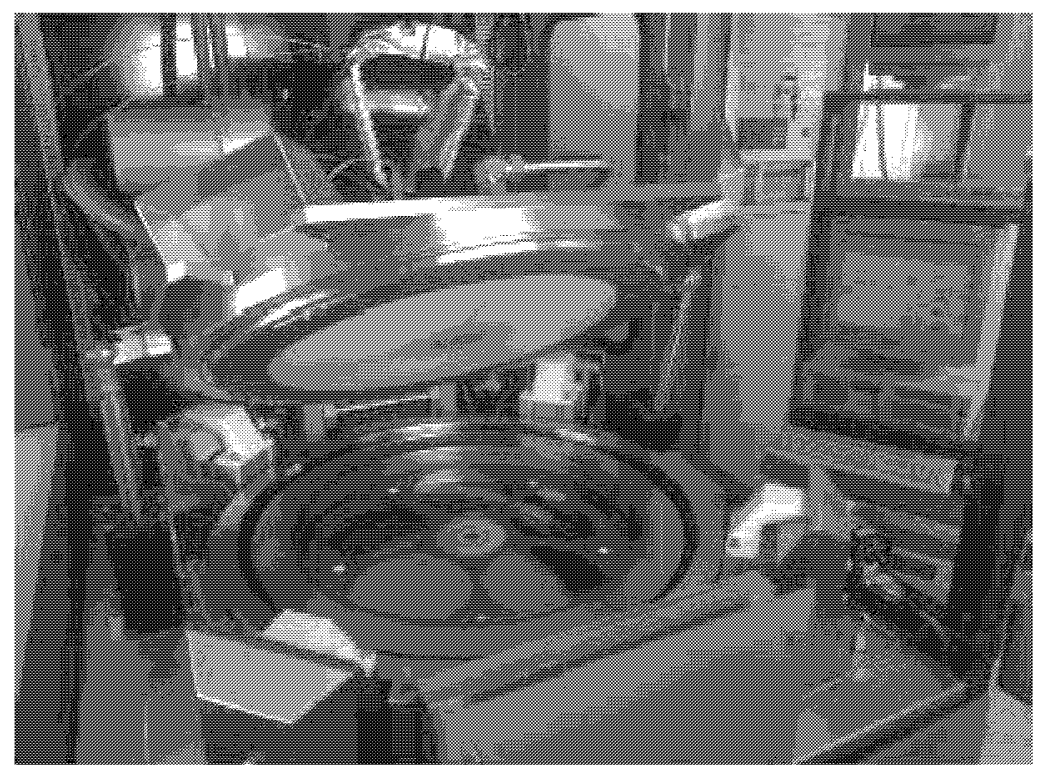

Fig. 5.2: $A L X T R O N$ 2600G3 planetary reactor ${ }^{\circledR}$. The five $6 "$ process wafers are clearly visible. The lid with the quartz plate is approx. $25 \mathrm{~mm}$ above the rotating substrate.

The used AIXTRON Planetary Reactor ${ }^{\circledR}$ of the $2600 \mathrm{G} 3$ series can handle five 6 -inch wafers simultaneously. New versions of this reactor handle up to $7 \times 6$ " wafers at the same time. As shown in Fig. 5.2 the wafers are placed on a coated graphite susceptor that rotates typically at $8 \mathrm{rpm}$ and carries five smaller plates (satellites) which rotate at $40 \mathrm{rpm}$ by gas foil rotation. The gas inlet is placed central above the reactor providing a pure horizontal gas flow direction which makes this reactor a radial flow system. A quartz nozzle is used to ensure good mixing and spatial distribution of the precursor vapor. The oxidizer gases enter the reactor just below 
the nozzle separate from the precursors in order to avoid premature reactions. The desorbed products as well as the non reacted molecules are transferred through a ring line, the so called collector ring, and gather into a cold trap. The reactor operates under low pressure at $\sim 2 \mathrm{mbar}$ in order to increase the gas diffusivity and prevent pre-reactions. The pumping system consists of a roots or booster pump and a rotary pump for pumping larger gas loads like the carrier gas. The deposition temperatures varied between $500-700^{\circ} \mathrm{C}$. The process conditions are summarized in Table 5.1.

\begin{tabular}{ll}
\hline Substrate temperature & $500-750^{\circ} \mathrm{C}$ \\
Reactor pressure & $1-3 \mathrm{mbar}$ \\
$\mathrm{O}_{2}$ flow rate & $50-500 \mathrm{sccm}$ \\
$\mathrm{N}_{2} \mathrm{O}$ flow rate & $50-500 \mathrm{sccm}$ \\
Liquid source feeding rate & $0.08 \mathrm{ml} / \mathrm{s}$ \\
Ceiling temperature & $190-290^{\circ} \mathrm{C}$ \\
Vaporization temperature & $225-245^{\circ} \mathrm{C}$ \\
Film thickness & $10-120 \mathrm{~nm}$ \\
\hline
\end{tabular}

Table 5.1: Typical deposition conditions for BST films

The homogenous heating of the graphite susceptor is achieved by infrared lamps positioned below the rotating disk. Throughout the susceptor the temperature variation was estimated to be $\pm 1 \%$ of the set value. The reactor ceiling is water cooled and is covered by an exchangeable quartz plate. The walls are also water cooled and their temperature is kept constant throughout the process. Ceiling and walls are kept at much lower temperatures compared to the susceptor in order to suppress unwanted deposition on these surfaces. Because it is not possible to completely avoid such effects the ceiling plate has to be frequently cleaned. The ceiling plate is coupled to the water cooled lid by an Ar/He mixture flowing in the gap between them. The distance $t_{\text {gap }}$ of the gap can be varied $\left(1 \mathrm{~mm}<t_{\text {gap }}<0 \mathrm{~mm}\right)$ so that a broad temperature range can be adjusted at the top of the reactor. Since Helium dissipates the heat better than Ar from the ceiling plate to the lid, the exact temperature is adjusted by the ratio of the two gases. The ceiling temperature was found to have a significant impact on the deposition homogeneity.

The susceptor temperature is controlled by two pyrometers at both sides of the susceptor. The measurement from the rear side provides the actual susceptor temperature and the pyrometer is always focused at the rotating graphite body. The second pyrometer monitors the temperature from the top. In this case, the instrument measures through the ceiling plate alternately on the graphite and the satellites so that a temperature profile of the wafer surface can be derived. Nevertheless, no reliable information about the wafer temperature can be retained in case of extensive secondary deposition at the ceiling. The most important issue in the adjustment of wafer surface temperature results from the bad thermal coupling of the satellites on the graphite susceptor because of the gas foil rotation principle (see Figs. $3.9 \mathrm{~b}$ and $3.14 \mathrm{f}$ ). As long as the process pressure is sufficient high, e.g. $>100 \mathrm{mbar}$, the surface temperature matches the susceptor temperature setpoint. At low pressure conditions the thermal coupling gets worse and the heat cannot be transferred from the massive graphite susceptor to the rotating satellites resulting in a colder wafer surface compared to the nominal susceptor temperature. Hence, the actual surface temperature may display large drifts 
compared to the susceptor temperature in the range of $20-50^{\circ} \mathrm{C}$ depending on the process temperature and pressure. The temperatures referred in this paper are exclusively nominal susceptor temperatures.

\subsection{Reactor performance}

The radial flow of the gases in the reactor leads to a deposition profile along the heated susceptor (Fig. 5.3). This behavior was examined using a non-rotated satellite. Immediately behind the nozzle, the growth is minimal and each precursor species requires a certain amount of thermal energy in order to decompose its organic components and participate in the growth process. The reactor and the process parameters are properly dimensioned so that the growth rate reaches a maximum just before the wafer position. The gliding of the precursors across the susceptor results in a linear depletion of the deposition rate across the wafer expressed in terms of the sinking incorporation rate of the individual precursors. The depletion gradient for the single elements is similar for all the used species ( $\mathrm{Ba}, \mathrm{Sr}, \mathrm{Ti})$ but the magnitude of the deposition rate of the individual precursors may be different for a given process window. According to the depletion profile across a non rotating wafer, the $\mathrm{Ba}$ and $\mathrm{Sr}$ precursors behave, as expected, similar. The efficiency of the Ti precursor is generally up to $25 \%$ higher compared to the group-II (Gr-II) chemicals but also displays the highest temperature sensitivity as can be seen in Fig. 5.4.

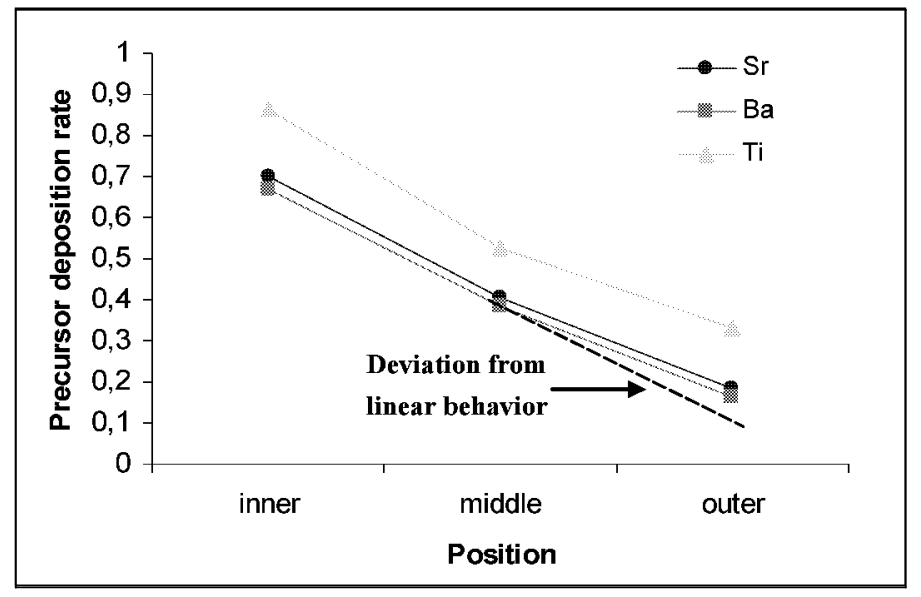

Fig. 5.3: Precursor deposition rate normalized for the middle position as a function of the distance to the nozzle $\left(T_{\text {growth }}=595^{\circ} \mathrm{C}\right)$. A slight deviation from the linear behavior cannot be completely avoided for any parameter set; nevertheless we obtain good homogeneity values across a 6" wafer (see also Fig. 5.8).

The profiles can be smoothed or shifted in the radial direction by adjusting the gas flow pattern, usually by changing the gas flow rate so that the linear depletion regime is exactly across the satellite position. Nevertheless, film growth is strongly disturbed by temperature gradients across the susceptor or satellites, that are most important at low temperatures, e.g. the stoichiometry value at the edge of the 6" wafer displays a 2-4\% deviation from the value at the central point. This effect is also present at higher deposition temperatures $\left(>580^{\circ} \mathrm{C}\right)$. By rotating the satellite it is possible to achieve a homogenous distribution of the growth rate, exactly as predicted by the simulation [41]. Thus, a variation of $1-2 \%$ in thickness and stoichiometry measured on rotated wafers can be considered as an excellent value.

The reduction of the thermal budget during deposition of high-k materials is highly desirable and one of the primary aims of this work was to investigate the feasibility of low temperature depositions. Additionally, the low temperature regime is targeted, since it enables conformal deposition over complex 3D structures [74]. For this, we performed depositions down to $460^{\circ} \mathrm{C}$, using similar process conditions as in the high $\mathrm{T}_{\text {growth }}$ regime. Fig. 5.4 presents the 
precursor efficiency for the examined temperature range from 655 to $460^{\circ} \mathrm{C}$. The efficiency of the precursor consumption is defined as the ratio of the quantity deposited on all 5 wafers to the amount of the individual precursor elements contained in the consumed liquid. As the rotating wafers are rather homogeneous, this average value is well defined and is an important factor for the costs of ownership of a MOCVD system. The observed average values of $45 \%$ for $\mathrm{Ti}$ and $35-40 \%$ for $\mathrm{Ba}$ and $\mathrm{Sr}$ for our standard growth processes are extraordinarily high compared to the reported values for conventional showerhead reactors $(\sim 3-10 \%)[1]$.

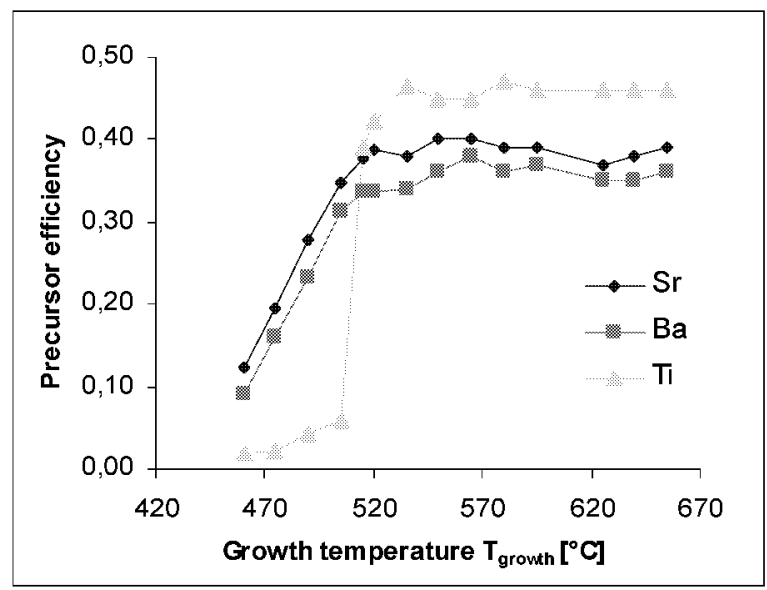

Fig. 5.4: Investigation of low temperature processing: precursor efficiency vs. susceptor temperature.

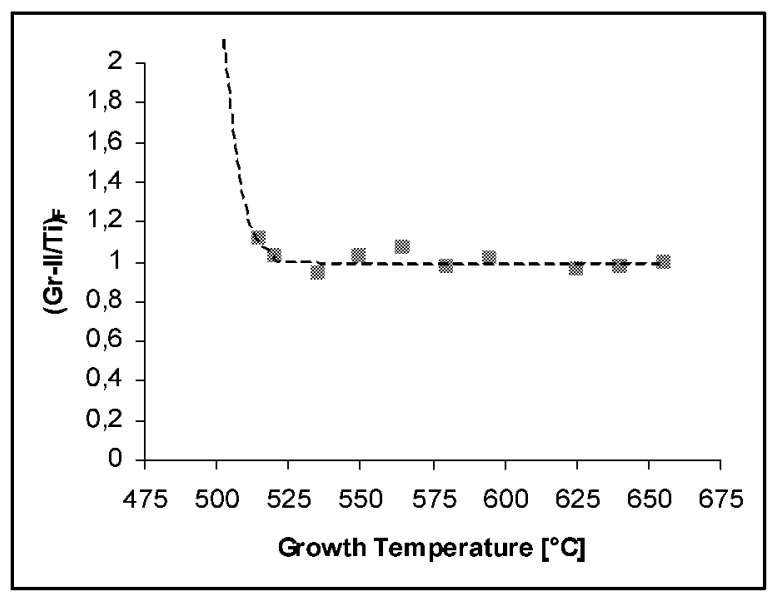

Fig. 5.5: Stoichiometry variation over growth temperature for a given liquid Gr-II/Ti ratio. Below $515^{\circ} \mathrm{C}$ the stoichiometry displays a strong deviation from the target ratio.

The efficiency of the incorporation of the Gr-II precursors decreased steadily below $515^{\circ} \mathrm{C}$. A similar behavior was observed by Kang at a 60 to $80^{\circ} \mathrm{C}$ lower temperature [80]. Up to now it is not understood, whether minor differences in the precursors, e.g. aging or the use of different solvents and/or stabilizers, or the reactor design and temperature calibration is the reason for this difference in the precursor behavior. The sinking of the precursor efficiency influences the film stoichiometry, especially below this given temperature point. This is shown in Fig. 5.5 for films grown between 670 and $460^{\circ} \mathrm{C}$ in $15^{\circ} \mathrm{C}$ temperature steps. For a given liquid stoichiometry at the LDS side, the film composition $(\mathrm{Ba}+\mathrm{Sr} / \mathrm{Ti})_{\mathrm{F}}$ was near unity for films grown above $580^{\circ} \mathrm{C}$. There is a slight variation of the stoichiometry with temperature, starting below $580^{\circ} \mathrm{C}$. The obtained strong decrease in the precursor efficiency below $515^{\circ} \mathrm{C}$ leads to a massive shift in the film stoichiometry, which is well out of the desirable range and is only presented dotted in Fig. 5.5 , e.g. at $505^{\circ} \mathrm{C}$ the $(\mathrm{Ba}+\mathrm{Sr} / \mathrm{Ti})_{\mathrm{F}}$ stoichiometry ratio was determined to 6.83 . As this fast decrease of the Ti efficiency cannot be reliably compensated by a change of the liquid stoichiometry, we concentrated on depositions at a minimum susceptor temperature of $520^{\circ} \mathrm{C}$ which corresponds to a wafer surface temperature of maximum $500^{\circ} \mathrm{C}$.

Generally, the variation of the film stoichiometry can be controlled by an adjustment of the liquid precursor as shown in Fig. 5.6. for four different deposition temperatures between $520^{\circ} \mathrm{C}$ and $655^{\circ} \mathrm{C}$. One can see that there is a roughly linear relation between liquid and film stoichiometry regardless of the deposition temperature. This is not surprising, since the efficiency of the precursors remain almost constant in this temperature regime, as it was previously shown in Fig. 5.4. The linear behavior enables rough composition control for the target stoichiometry. One can see that a 10 to $40 \%$ excess in Gr-II chemicals is necessary to 
approximate the stoichiometric composition in the film. However, this plot displays the data points from a large number of different runs and shows some scattering, because the data is strongly dependent on the actual precursors used and the deposition parameters, that have to be stable throughout the series of experiments. Especially after changing one of the precursor tanks, a recalibration of the plots is needed to ensure the correct readout. Change of liquid stoichiometry is, however, a time consuming issue connected with a high consumption of costly precursors and should be practised only when moving large steps between low and high temperature regime. Small changes can be easier achieved by varying other process parameters like gas flow, oxygen partial pressure and ceiling temperature (Chapter 5.2).

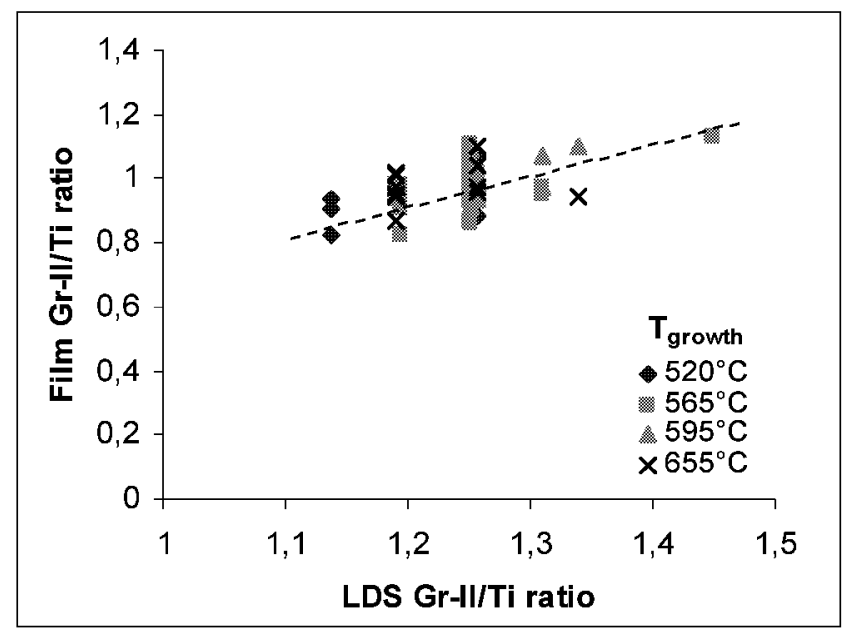

Fig. 5.6: Linearity between liquid and film stoichiometry enables stoichiometry control. The data is collected from a large number of runs. There is a significant scattering of the data points that depict the variations of the process parameters.

The importance of thickness scaling of the dielectric in DRAM capacitors was already mentioned in the introductory chapter. The resulting increase in capacitance through thickness down-scaling is the basic parameter to preserve a certain capacitance density during the shrinking of the bit cell dimensions. Without in-situ control the simplest way to scale the films thickness is just by changing the deposition time and this method can be applied as long as there is a very stable growth at every deposition temperature. Fig. 5.7 shows the results of the thickness scaling as a function of the deposition time for samples grown at $595^{\circ} \mathrm{C}$.

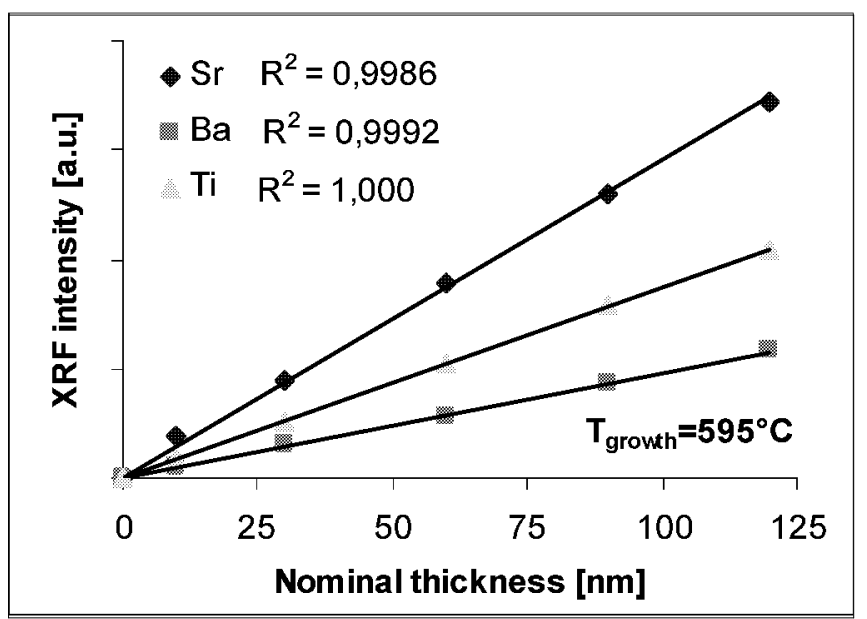

Fig. 5.7: Thickness scaling over the nominal thickness performed through variation of the deposition time for films grown at $595^{\circ} \mathrm{C}$. 
The results are presented in terms of the XRF intensity which is a linear function of the element concentration in the film (Section 4.1.2). The line fits match excellent the acquired points with a correlation factor very close to unity and the stoichiometry of the samples during down scaling remains additionally very stable: $(\mathrm{Ba}+\mathrm{Sr} / \mathrm{Ti})_{\mathrm{F}}=0.96 \pm 2 \%$. Nevertheless, there is a deviation from this behavior as the films become very thin $(<10 \mathrm{~nm})$, where the measuring signal is extremely low so that the measurement precision is not guaranteed. As a result, the measured stoichiometry of ultra thin films is afflicted with large error margins.
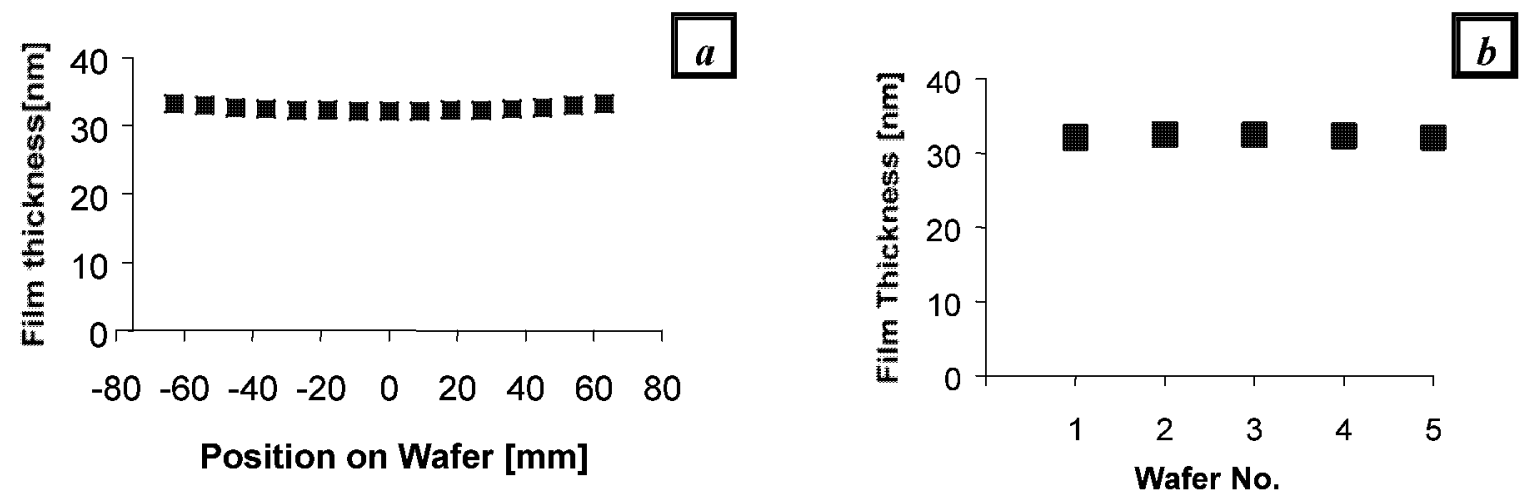

Fig. 5.8: Ellipsometry measurements of the film thickness on $30 \mathrm{~nm}$ Ti-rich films: a) Thickness variation across a 6" wafer b) Thickness variation between 5 simultaneously process wafers

As can be seen in Fig. 5.4, deposition takes place above $520^{\circ} \mathrm{C}$ with a widely constant deposition rate, which is approximately $2.5 \mathrm{~nm} / \mathrm{min}$ and indicates that the whole range is still under mass transfer limiting conditions. This value is rather low compared to other reactor types, however it must be considered that five substrates can be processed at once, which corresponds to a deposition rate around $10 \mathrm{~nm} / \mathrm{min}$. Finally, XRF demonstrated a very good homogeneity of stoichiometry and film thickness over 6" wafers which is due to the gas-foil rotation principle. These results are further supported by ellipsometry measurements, that yield, for example, a thickness of $32,4 \pm 0,4 \mathrm{~nm}$ and a stoichiometry variation of $53,8 \pm 0,3$ at $\%$ Ti. Similar small differences were observed between the five simultaneously processed wafers (Fig. 5.8) [86].

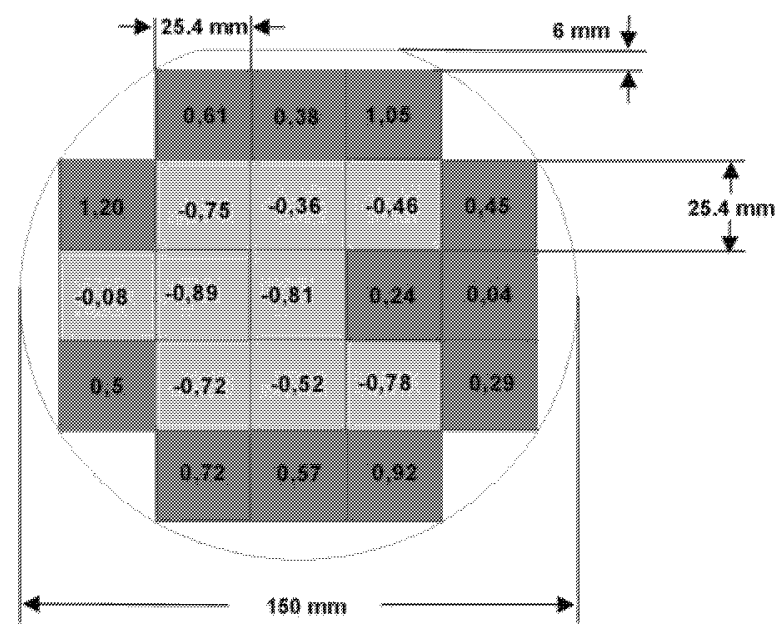

Fig. 5.9: Mapping the thickness across a 6 " wafer. The deviations from the average value are given in $\%$. An average thickness of $62.9 \mathrm{~nm}$ was obtained and the standard deviation from this value was determined to $0.65 \%$. 
An additional mapping of thickness and stoichiometry uniformity was performed by XRF. A 6-inch wafer was divided into 21 one square inch samples after BST deposition. Each sample was analyzed in terms of thickness and composition. The average thickness was $62.9 \mathrm{~nm}$ with a standard deviation variation of $0.65 \%$ and the distribution, which indicates a depletion in the middle of the wafer is shown in Fig. 5.9. The $\mathrm{Ba}$ and $\mathrm{Sr}$ concentration remained constant all over the wafer within these uncertainties, which are determined by the precision of the XRF for these elements. For $\mathrm{Ti}$, however, we observed a depletion from the wafer edge to the middle which correlates to the thickness map in Fig. 5.9 [87]. This depletion may be caused by the better thermal coupling of the wafer edge to the rotating satellite.

\subsection{Optimization of process parameters for BST}

The growth process of BST in the AIXTRON 2600G3 reactor has been optimized within a multi dimensional parameter space. The most important parameters that control the deposition are the deposition temperature, which was already mentioned in the previous section, the gasand especially the oxidizer- flow rate, the process pressure and the vaporizer temperature.

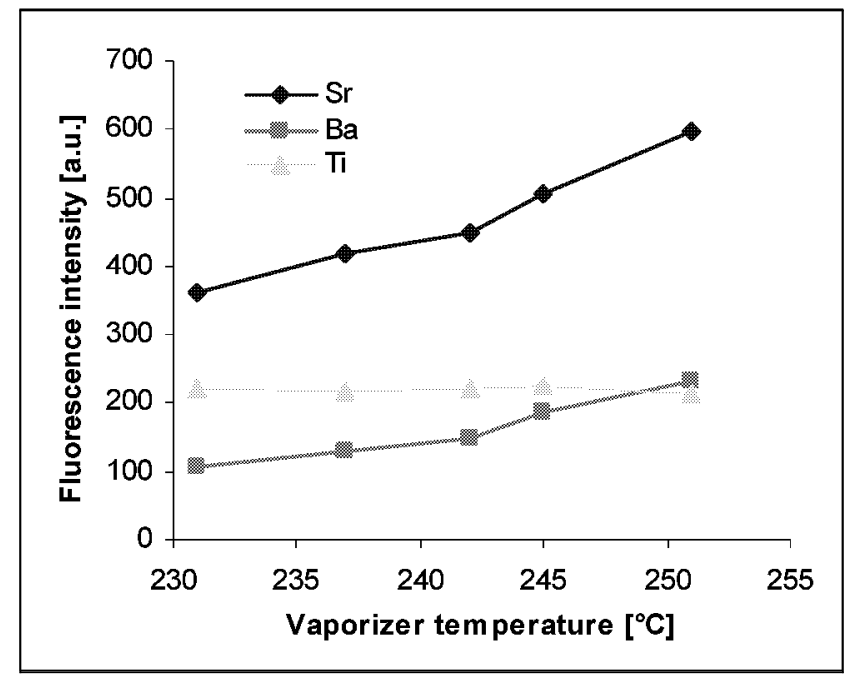

Fig. 5.10: Influence of the vaporizer temperature on the precursor incorporation expressed through the fluorescence intensity. A significant influence on the group-II elements is observed, in contrast to Ti which remains stable.

Evaporation of the liquid precursors is a critical part in the growth process. Low vaporizer temperature could lead to partial evaporation and contamination of the vaporizer chamber. On the other hand, too high temperatures can cause precursor decomposition, so that a reasonable temperature value has to be found. A series of experiments was performed while the vaporizer temperature was varied between 231 and $251^{\circ} \mathrm{C}$ (Fig. 5.10). The vaporizer temperature has a prominent influence on the Gr-II elements that is depicted in the intensity of the fluorescence signal, in contrast to $\mathrm{Ti}$, which remained practically unchanged. Consequently, the vaporizer temperature was set to a mean value of $240^{\circ} \mathrm{C}$ in order to avoid any decomposition effects and increased maintenance actions. However, the details may depend on the quality of the precursors and a different behavior had been observed for an early charge.

Low process pressure is crucial to ensure a sufficient large free path length of the precursor molecules and suppress unwanted pre-reactions in the gas phase. Fig. 5.11a-b show the temperature dependence of the observed film stoichiometry for three different process pressures by using otherwise the same process parameters. Although the difference between 1 and $2.5 \mathrm{mbar}$ is not significant, besides a sharper decrease of the Gr-II/Ti ratio at $1 \mathrm{mbar}$ towards higher temperatures, we observe at 10 mbar a huge shift from the previous behavior, as the overall precursor efficiency drops dramatically. Finally, the Ti precursor is affected 
much more than the Gr-II chemicals and the Gr-II/Ti ratio trends towards zero. It should be mentioned that the large deviation in the behavior Fig. 5.11b, compared Fig. 5.5, can be explained in terms of the different precursors charges and the changes in the nozzle and gas mixing system. This is an indication that minor changes in the reactor design and/or the chemical components may lead to strong drifts in the growth process. Consequently, a detailed check has to be performed after each change in the system.
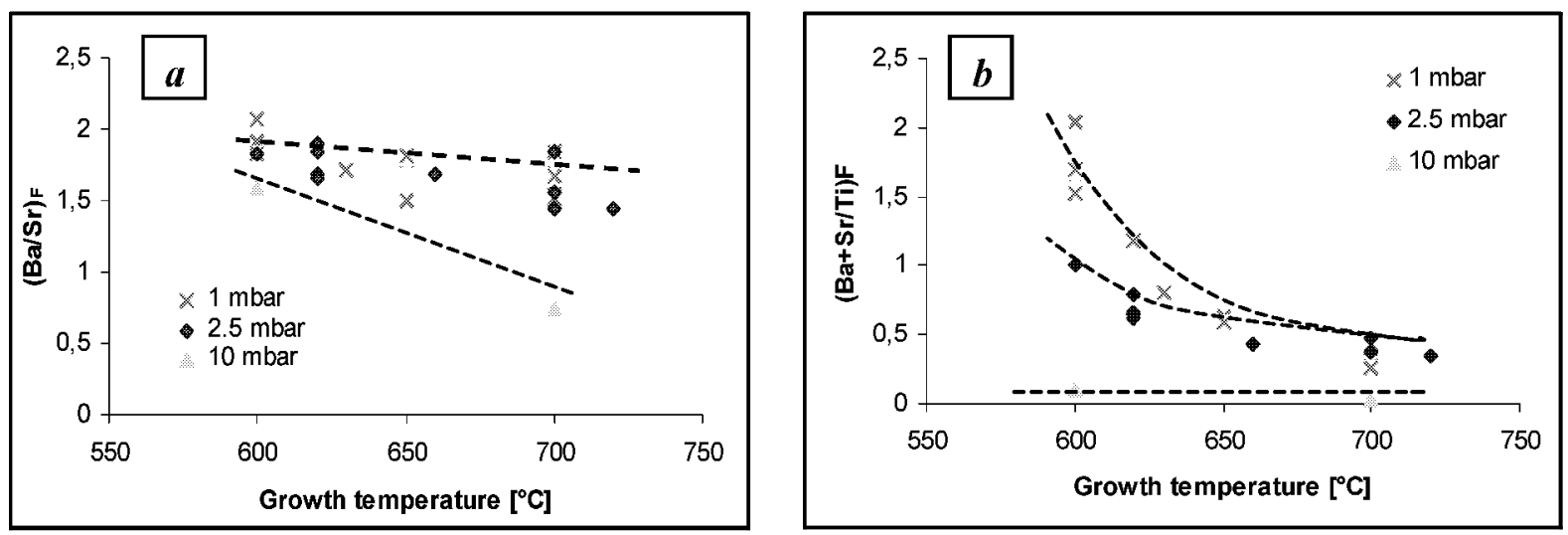

Fig. 5.11: Film stoichiometry at three different pressures a) $(\mathrm{Ba} / \mathrm{Sr})_{F}$ ratio vs. growth temperature b) $(G r-I L / T i)_{F}$ ratio vs. growth temperature.

The magnitude of the total flow rate is mainly dictated by the geometry of the reactor and by the nozzle principle and is properly adjusted, according to the Reynolds number of the carrier gas and the oxidizers, so that a laminar flow is established (Chapter 3.1.4). For the given reactor and nozzle configuration the ratio of the Ar carrier to the oxidizer flow was calculated to approx. 25:1. The flow pattern in the reactor at a given process pressure does not change dramatically within the focused temperature region, and only minor adjustments are necessary.

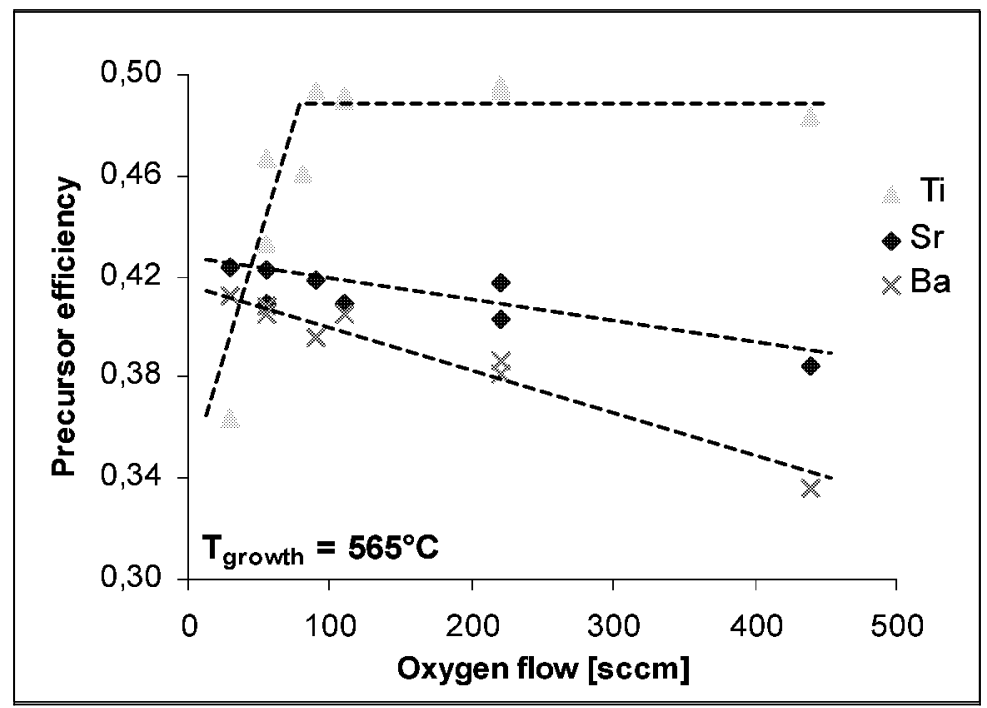

Fig. 5.12: Oxidizer influence on the precursor efficiency at $565^{\circ} \mathrm{C}$. There is a strong impact on the $T i$ efficiency for an oxygen flow below $100 \mathrm{sccm}$. The Gr-II elements display a slight drop with increasing oxygen flow.

In contrast to the Ar flow rate, the amount of the oxidizer in the gas flow was found to have a significant influence in the incorporation efficiency of the precursors. Both $\mathrm{O}_{2}$ and $\mathrm{N}_{2} \mathrm{O}$ were 
generally used as oxidizers and $\mathrm{N}_{2} \mathrm{O}$ is considered to be more reactive than $\mathrm{O}_{2}$ in the reaction with the precursors. However, this is only valid at higher temperatures. At $565^{\circ} \mathrm{C}, \mathrm{N}_{2} \mathrm{O}$ shows no influence and pure oxygen was applied. Fig. 5.12 displays the effect of the oxygen flow at $565^{\circ} \mathrm{C}$ on the precursor efficiency. While the Gr-II elements show just a slight drop with an increasing oxygen flow, the Ti displays a much more prominent dependence with a clear maximum at $100 \mathrm{sccm}$. Consequently, the oxygen flow rate was set to this value. Experiments at elevated growth temperatures did not display significant deviations from this behavior. This change was also proven to be an excellent way to trim the stoichiometry, as long as the flow values are taken from the linear region of the graph $(>100 \mathrm{sccm})$. A low oxygen flow results into off-stoichiometric nonreproducible films.

The previous graphs show some general trends, however, most of the parameters are coupled, so that they cannot be varied independently. The final fine tuning of the process parameters (e.g. temperature, oxidizer flow, ceiling temperature) was therefore attempted by a statistical variation of these parameters within a limited parameter space, the so called Design Of Experiment (DOE) approach. The observed trends were not very significant as not all parameters could be controlled with sufficient precision at the time, especially the reactor pressure, the temperature of the ceiling and the precursor evaporation conditions. In addition, production level reproducibility seems only manageable with the implementation of a wafer handler, so that the reactor can run continuously and does not have to be opened for loading. The investigations of the film properties in the following chapter were performed on samples that were deposited using the optimized process conditions presented here. 


\section{$6 \quad$ Properties of BST thin films}

In the present chapter the properties of thin films grown within a multi-dimensional parameter space including deposition temperature, stoichiometry, substrate quality and film thickness will be presented. As these strongly interrelated parameters cannot be discussed in detail a more straightforward guideline of presentation is chosen.

In the first part of the chapter the structure and morphology of the films is discussed, beginning with a description of the nucleation and growth studies of BST. Ultra thin films below one mono-layer and up to $5 \mathrm{~nm}$ were deposited, in order to investigate the seed growth. These studies where performed using atomic force microscopy (AFM). The further growth of the closed films was followed up to a thickness of $130 \mathrm{~nm}$ and different measurement methods were applied for the determination of the film thickness. Next, the film microstructure will be discussed in detail for films of $30 \mathrm{~nm}$ thickness, that are close to the stoichiometric composition or slightly Ti-rich. The growth temperature is considered a major parameter in these studies since a low thermal budget is desirable. On the other hand, there is a degradation of the film properties observed in the low temperature regime $\left(\leq 565^{\circ} \mathrm{C}\right)$ as compared to high temperatures $\left(>595^{\circ} \mathrm{C}\right)$ and a compromise must be found for the final application. All mentioned temperatures will be exclusively susceptor temperatures (see Chapter 5). Subsequently, the influence of the stoichiometry and of the substrate type on the microstructure will be presented.

In the second part of the chapter it will be reported on the electrical properties of BST thin films in the thickness range between 8 and $130 \mathrm{~nm}$. The permittivity measurements will be discussed in terms of the "dead layer" model and the dielectric films will be tested for the application in the Gbit DRAM technology. Finally, the chapter is concluded with a short summary of the structure property relationship which can be deduced from the wide range of data.

\subsection{Structure and morphology of the films}

The nucleation and growth behavior of BST films on Pt electrodes was investigated within a wide temperature range, from 520 to $655^{\circ} \mathrm{C}$. Generally, XRD analysis reveals amorphous films below a growth temperature of $520^{\circ} \mathrm{C}$. The films at $520^{\circ} \mathrm{C}$ show poor crystallinity and grazing incidence XRD was necessary to reveal the week BST line pattern. The deposited films at $565^{\circ} \mathrm{C}$ are polycrystalline. Finally, above $595^{\circ} \mathrm{C}$ a transition takes place from polycrystalline to (100)-textured films. The microstructure was examined extensively using a large spectrum of additional analyzing techniques. FTIR was used to determine additional foreign phases. SIMS and SNMS were utilized to control the quality of the interface and element interdiffusion. Finally, SEM, AFM and TEM were widely applied for the morphological characterization of the films.

Table 6.1 presents an overview of the parameter space that will be discussed in the following. For a certain position in the table the performed investigations are displayed. The columns are divided according to the composition of the films and the rows depict the growth temperature. Since we handle a multiparameter space, different symbols are inserted in each table cell to show the applied analytical methods. A film with a standard thickness of $30 \mathrm{~nm}$ is represented by a large dot. Other symbols represent the existence of a thickness series (gray background), FTIR investigations $(\diamond)$, microscopy pictures, e.g. AFM, SEM or HRTEM (ם), and depth $\operatorname{profiling}(\mathbf{\Delta})$. 


\begin{tabular}{|l|c|c|c|c|c|c|c|}
\hline Temp. & \multicolumn{7}{|c|}{ Stoichiometry (Gr-II/Ti) } \\
\hline & $<0.90$ & $0.91-0.94$ & $0.95-0.98$ & $0.99-1.01$ & $1.02-1.05$ & $1.06-1.09$ & $>1.10$ \\
\hline $670^{\circ} \mathrm{C}$ & & & & $\bullet \square$ & & & \\
\hline $655^{\circ} \mathrm{C}$ & $\bullet \diamond \Delta$ & $\bullet \square$ & $\bullet \square$ & $\bullet$ & $\bullet \square \Delta$ & $\bullet \square$ & \\
\hline $625^{\circ} \mathrm{C}$ & & $\bullet \diamond \square$ & $\bullet \diamond \square$ & $\bullet \diamond \Delta$ & $\bullet \diamond \square$ & & \\
\hline $595^{\circ} \mathrm{C}$ & & $\bullet \square$ & $\bullet \diamond$ & $\bullet \square \wedge$ & $\bullet \square$ & $\bullet \square$ & $\bullet$ \\
\hline $580^{\circ} \mathrm{C}$ & & & $\bullet \diamond \square$ & $\bullet$ & $\bullet$ & & \\
\hline $565^{\circ} \mathrm{C}$ & $\bullet$ & $\bullet \diamond$ & $\bullet \diamond \square$ & $\bullet \diamond \square \Delta$ & $\bullet \square$ & $\bullet \diamond$ & $\bullet$ \\
\hline $520^{\circ} \mathrm{C}$ & & $\square$ & & & $\bullet \diamond \square$ & & $\bullet \square$ \\
\hline
\end{tabular}

Table 6.1: The complete range of processed films and the analytical methods that were used for morphological characterization. The dots $(\bullet)$ represent films around $30 \mathrm{~nm}$ and a gray background stands for the existence of a thickness series with at least three elements. Other symbols represent FTIR $(\diamond)$, microscopy $(\square)$ and SIMS/SNMS analysis $(\boldsymbol{\Delta})$.

\subsubsection{Nucleation and Growth}

In the present section we will present some examples of the nucleation of BST thin films. An atomic force microscope (AFM) from SIS was used for routine investigations of the film surface in order to reveal the evolution of the surface morphology during nucleation and growth. The influence of the thickness on the film morphology was further examined in a series of SEM pictures.

\section{a) Nucleation on (111) Pt}

Fig. 6.1 shows the topology of a standard substrate supplied by aixACCT. The wafers consist of a $\mathrm{Pt} / \mathrm{TiO}_{\mathrm{x}} / \mathrm{SiO}_{2} / \mathrm{Si}$ stack with a $10-15 \mathrm{~nm} \mathrm{TiO}_{\mathrm{x}}$ adhesion layer and a $100 \mathrm{~nm} \mathrm{Pt}$ film. These substrates routinely undergo a RTA processing at $600^{\circ} \mathrm{C}$ in oxygen ambient before BST deposition, that results into a small increase of the surface roughness, which was initially around $1 \mathrm{~nm}$. After the RTA step, Pt film remains flat with an RMS of $1.19 \mathrm{~nm}$, even though there are some singular peaks that might indicate hillock formation during the annealing procedure (Fig. 6.2). Hillocks can be preferred nucleation spots related to the growth of larger grains compared to the rest of the film. This is also documented in some SEM figures that will be discussed in detail in the next sections. These structures may vary to some extend for different charges of wafers used over the period of investigations and could not be continuously controlled. Hence, there is some uncertainty about some inhomogeneous structures observed on different micrographs.

The following series of AFM images shows the surface topography of BST films. The selected sections have all the same size $(3.46 \mu \mathrm{m} \times 3.46 \mu \mathrm{m})$ for ease of comparison. The relative height, however, is scaled for each image separately and is explained at the right hand side of the figures. Additional high resolution scans with a different AFM tool with a scanned area $1 \mu \mathrm{m}$ by $1 \mu \mathrm{m}$ were performed to reveal the details of the structures. The pictures (Fig. 6.3-Fig. 6.7) show the growth at a temperature of $565^{\circ} \mathrm{C}$ at deposition times of $11 \mathrm{~s}, 33 \mathrm{~s}$ and $110 \mathrm{~s}$, which correlates to an average thickness of $0.5 \mathrm{~nm}, 1.5 \mathrm{~nm}$ and $5 \mathrm{~nm}$. Time control errors $( \pm 4 \mathrm{~s})$ must be considered, because of the unreliable switching times of the LDS. The nucleation studies were performed exclusively on RTA processed aix $A C C T$ substrates. These ultra thin films were too thin to be measured by XRF, as shown in Chapter 4, however, we 
can expect an excess of $\mathrm{Ti}$ in the films according to the adjusted liquid precursor ratio and measurements at thicker films deposited with the same process parameters.

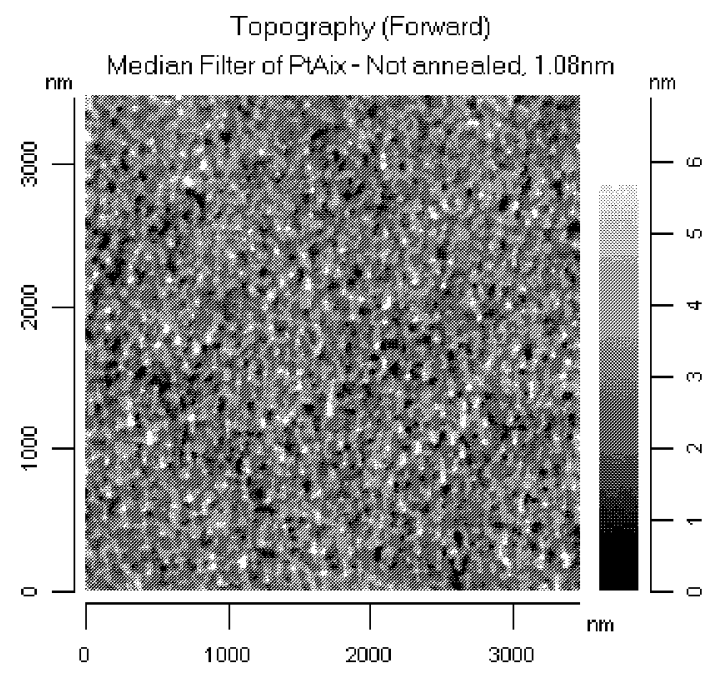

Fig. 6.1: AFM picture of the surface of the platinum wafer before RTA processing. The surface is flat with an rms roughness around Inm (supplier: aixACCT).

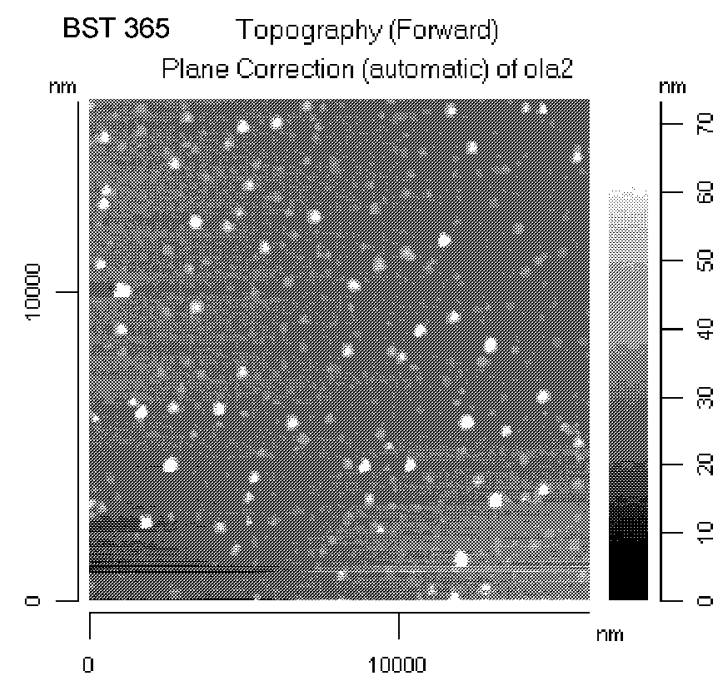

Fig. 6.3: $A F M$ scan of a $15 \mu m \times 15 \mu m$ area. The nominal BST thickness is $0.5 \mathrm{~nm} ; A$ random distribution of several large structures is observed.

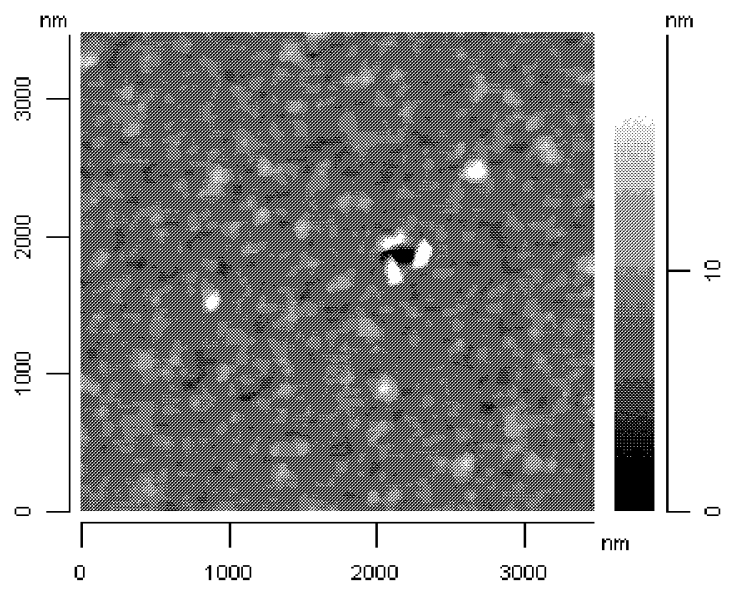

Fig. 6.2: $A F M$ picture of the surface of the platinum electrode before BST deposition. The observed area appears generally flat with an indication for some hillocks. The average value for the RMS roughness was determined to $1.19 \mathrm{~nm}$ (wafer supplied by aixACCT).

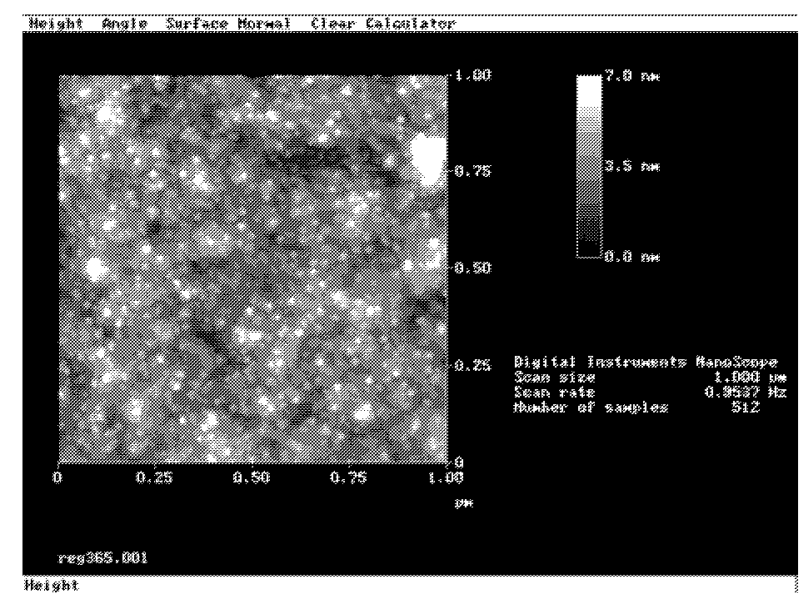

Fig. 6.4: Seed growth on (111) Pt. The BST thickness is $0.5 \mathrm{~nm}$ : The high resolution scan reveals small spots with a size of $10-20 \mathrm{~nm}$. These might represent the stable nuclei on platinum (HR scan of sample in Fig. 6.3).

Fig. 6.4 shows a high resolution AFM scan of the first deposition step (also see Fig. 6.3), which has an average thickness of $0.5 \mathrm{~nm}$. A large number of small structures can be found on the selected area that correspond to single seeds. These have a size of $10-20 \mathrm{~nm}$ and are situated directly on the platinum surface. There is indication for a non-random agglomeration 
of these seeds which might lead to the formation of larger structures (see right hand side of the scan). This may be related to the existence of defects in the Pt substrate surface (hillocks) that lead to a preferred nucleation, which finally causes the formation of larger crystallites. However, a look at the scan displaying a larger area (Fig. 6.3) of the same sample reveals several randomly distributed large structures, and particle contamination cannot be ruled out. The observation of problems in the evaporator system in the initial deposition phase could explain this large number of particles, that are often not visible in thicker films. These problems were avoided after process modifications by the end of the studies.

Fig. 6.5 and Fig. 6.6 show the next step in this series with an average thickness of $1.5 \mathrm{~nm}$ in two different resolutions. Compared to the naked substrate, an increased surface roughness with a value of $2.8 \mathrm{~nm}$ is observed. The comparison of these figures with Fig. 6.4 leads to the conclusion that the seeds have grown together in the time interval between the two steps and large structures with a size between $50-200 \mathrm{~nm}$ are now visible. These structures are significantly larger than the grain size determined by SEM observations which is not resolvable here. The HR scan in Fig. 6.6 additionally reveals layered BST patterns in some grains and demonstrates that the $1.5 \mathrm{~nm}$ film can be considered as a continuous layer.

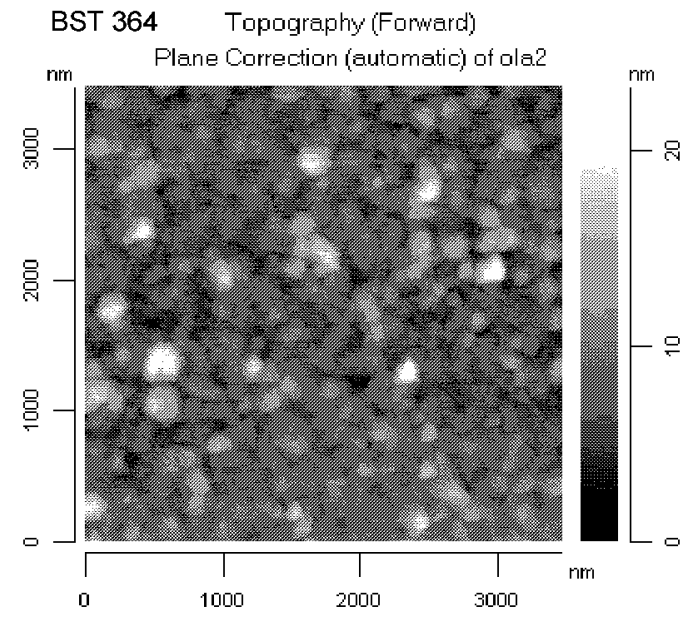

Fig. 6.5: AFM scan of the BST surface (thickness 1,5nm). The RMS value is $2.8 \mathrm{~nm}$. The seeds have grown and many small structures appear.

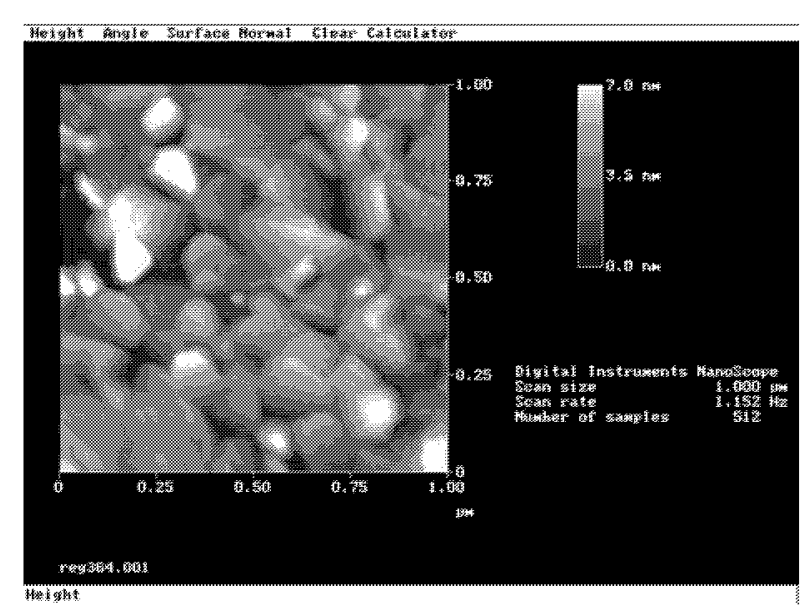

Fig. 6.6: $H R-A F M$ scan of a $1.5 \mathrm{~nm}$ BST layer. The film seems closed and irregular grains with a size of 50-200nm are visible.

The next deposition step at $5 \mathrm{~nm}$ displays a closed homogeneous film with a surface roughness similar to the preceding step, e.g. 3.2nm (Fig. 6.7). The size of the observed structures displays a slight increase and is estimated between $100-300 \mathrm{~nm}$.

A similar trend has been observed for both higher and lower deposition temperatures. Fig. 6.8 and Fig. 6.9 depict the topology of films after the nucleation step at $520^{\circ} \mathrm{C}$ and $655^{\circ} \mathrm{C}$, respectively. The film thickness is approx. $1.5 \mathrm{~nm}$. Both films appear closed with a comparable surface morphology. The mean surface roughness was determined to $1.5 \mathrm{~nm}$ for the film grown at $520^{\circ} \mathrm{C}$ and $1.9 \mathrm{~nm}$ for the film grown at $655^{\circ} \mathrm{C}$. There are some single peaks visible on the scans, that are attributed to irregular grain growth as discussed above. 

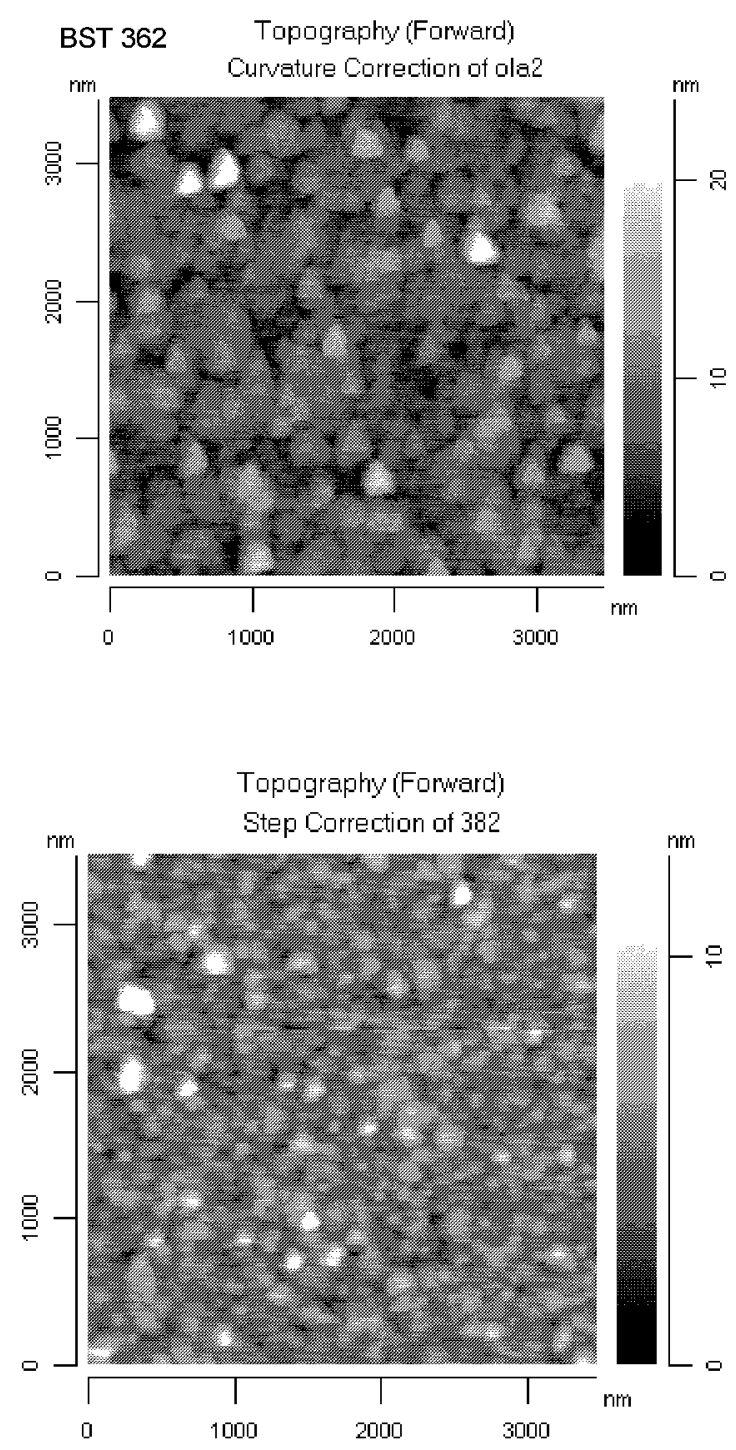

Fig. 6.8: $A F M$ scan of the surface of a $1.5 \mathrm{~nm}$ thin film grown at $520^{\circ} \mathrm{C}$; the $R M S$ roughness is $1.5 \mathrm{~nm}$.
Fig. 6.7: AFM scan of a BST film. The average thickness is 5nm and the roughness is $3.2 \mathrm{~nm}$. We observe a further growing of the structures and their size is now estimated at $100-300 \mathrm{~nm}$.

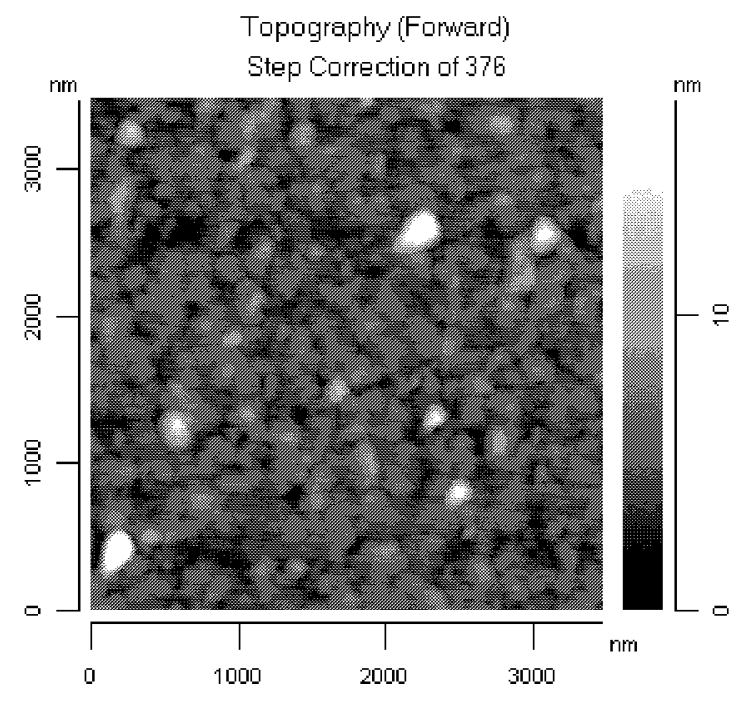

Fig. 6.9: AFM scan of the surface of a $1.5 \mathrm{~nm}$ thin film grown at $655^{\circ} \mathrm{C}$; the $R M S$ roughness is $1.9 \mathrm{~nm}$.

Concluding, an increase of the surface roughness could be observed within the nucleation stage and is summarized in Table 6.2. The initial low surface roughness of the $\mathrm{Pt}$ surface of approx. $1.2 \mathrm{~nm}$ increases to $2.8 \mathrm{~nm}$ for the $1.5 \mathrm{~nm}$ film and displays only a small increase to the next growth step, e.g. $3.2 \mathrm{~nm}$ for the $5 \mathrm{~nm}$ film grown at $565^{\circ} \mathrm{C}$. Within the precision and reliability of our measurements, we do not observe any significant differences in the nucleation behavior at different temperatures and the films appear already closed beyond a film thickness of $1.5 \mathrm{~nm}$. Local inhomogeneities in the film growth may result from larger grains or eventually grain clusters, as already mentioned. It is still not understood whether this effect is a function of the Pt quality or results from the growth behavior of BST. EDX investigations and high resolution AFM scans would be helpful to find out whether these peaks are caused by underlying platinum hillocks. Due to the use of many different Pt substrates some variation in the hillock density cannot be excluded. 


\begin{tabular}{c|cccc}
\hline & \multicolumn{4}{|c}{ Growth Temperature } \\
Film thickness & $\mathbf{5 2 0}^{\circ} \mathbf{C}$ & $\mathbf{5 6 5}^{\circ} \mathbf{C}$ & $\mathbf{5 9 5}^{\circ} \mathbf{C}$ & $\mathbf{6 5 5}^{\circ} \mathbf{C}$ \\
\hline $1.5 \mathrm{~nm}$ & 1.5 & 2.8 & $(3.7)$ & 1.9 \\
$5 \mathrm{~nm}$ & 9.7 & 3.2 & 2.5 & 1.8 \\
$30 \mathrm{~nm}$ & 16.6 & 3.8 & 2.2 & 3.1 \\
\hline
\end{tabular}

Table 6.2: Mean surface roughness (rms) during nucleation and film growth. The values are given in $\mathrm{nm}$.

\section{b) Film growth}

Thickness scaling is very important from the DRAM application point of view and special care has to be taken in order to prevent a shift of the deposition process while varying the thickness. On the other hand, many of the properties strongly depend on the film thickness. This is especially true for the electrical properties. Thus, it is desirable to investigate the thickness property relations with great precision to describe the behavior of the dielectric. Therefore we examined the microstructure as a function of the film thickness in some detail. Different thickness measurement techniques were applied and the results are compared. It is generally quite difficult to make comparisons to other published work because of the thickness dependence of many film properties.

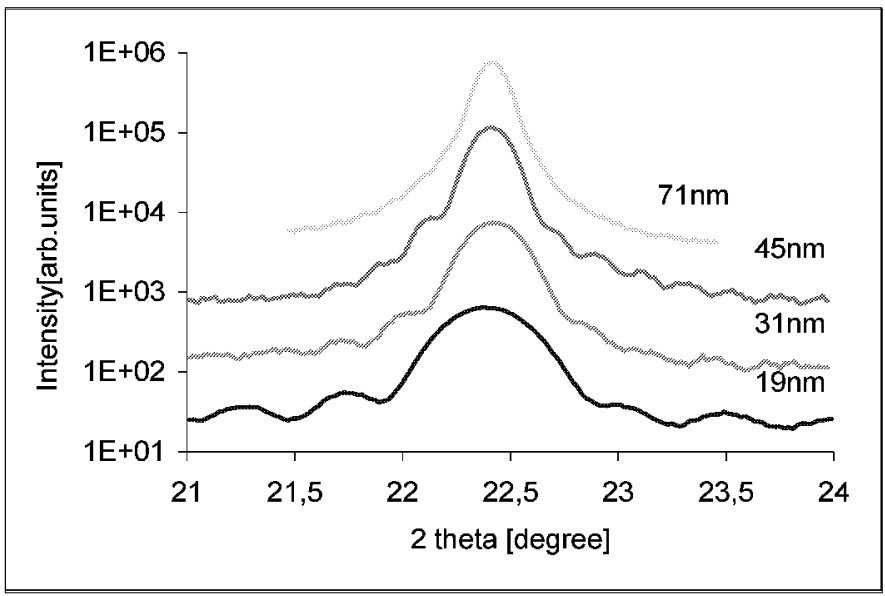

Fig. 6.10: Thickness oscillations close to the (100)-reflection of Ti-rich samples grown at $655^{\circ} \mathrm{C}$. The calculated thickness matches other measurement techniques like XRF and DEKTAK.

For samples grown at high temperatures thickness fringes are clearly visible in high resolution XRD scans. This is displayed for the (100) reflections of films between $19 \mathrm{~nm}$ and $71 \mathrm{~nm}$ at $655^{\circ} \mathrm{C}$ (Fig. 6.10). The thickness oscillations are quite strong for the thin samples and become weaker with increasing thickness. However, they are still visible for the $71 \mathrm{~nm}$ sample. On the other hand, the peaks become broader with decreasing thickness; these features would be more clear in a linear intensity scale. The wavelength of the oscillations $\Delta \theta$ yields immediately the film thickness $d$ according to the following equation:

$$
d=\frac{\lambda}{2 \cdot \Delta \theta \cdot \cos \theta}
$$

where $\lambda$ is the wavelength of the $\mathrm{Cu}-\mathrm{K} \alpha$ radiation used. This method provides us with the physical thickness of the examined films and can be used as a comparison or calibration to the 
other thickness measurement methods used, like XRF analysis and profile scanning using a DEKTAK tool. The results of this comparison are summarized in Table 6.3. The obtained sample thickness is in very good agreement with the XRF estimation using the theoretical density of stoichiometric bulk BST of $\rho_{\mathrm{BST}}=5.758 \mathrm{~g} / \mathrm{cm}^{3}$ and the thickness measured using a DEKTAK profilometer. The deviation of the values measured by DEKTAK for the $71 \mathrm{~nm}$ samples is in the range of the measurement precision, that is $\sim 10 \%$ for this kind of measurement, which is limited by the surface roughness. Both of the other techniques average the measured signal over a large surface area and are not influenced by local thickness variations.

\begin{tabular}{cccc}
\hline $\begin{array}{c}\text { Nominal } \\
\text { thickness [nm] }\end{array}$ & DEKTAK & XRF & $\begin{array}{c}\text { Thickness } \\
\text { fringes }\end{array}$ \\
\hline $\mathbf{2 0}$ & 20.3 & 19.0 & 19 \\
$\mathbf{3 0}$ & 31.7 & 30.7 & 31 \\
$\mathbf{4 5}$ & 48.0 & 44.5 & 45 \\
$\mathbf{7 0}$ & 80.5 & 70.9 & 70 \\
\hline
\end{tabular}

Table 6.3: Comparison between different thickness measurement techniques. All three methods present comparable results. The thickness values are given in $\mathrm{nm}$.

After the observation of the growth of closed films from $1.5 \mathrm{~nm}$ to $5 \mathrm{~nm}$ the following AFM images present a larger step in the growth to a thickness of $30 \mathrm{~nm}$. The surface morphology is shown after different deposition temperatures: $520^{\circ} \mathrm{C}$ (Fig. 6.11 ), $565^{\circ} \mathrm{C}$ (Fig. 6.12 ), $595^{\circ} \mathrm{C}$ (Fig. 6.13) and $655^{\circ} \mathrm{C}$ (Fig. 6.14). The films are prepared under similar process conditions with a deposition time of $660 \mathrm{~s}$ and an evaporation rate of $0.08 \mathrm{ml} / \mathrm{min}$.

Although the seed growth at different temperatures does not display significant differences, there is a large difference in the morphology between the films deposited at $520^{\circ} \mathrm{C}$ and the fully crystallized films obtained at $565^{\circ} \mathrm{C}$. The BST layer deposited at $520^{\circ} \mathrm{C}$ looks very homogeneous on a macroscopic scale with random structures of the size $50-200 \mathrm{~nm}$, that cause relative high surface roughness of $16.6 \mathrm{~nm}$. This granularity is a surprising result considering the low roughness for the $1.5 \mathrm{~nm}$ thin film (Fig. 6.8). It is possible that the continuous growth is disturbed from the large amounts of carbonates contained in the film. The strong carbonate concentration in the low deposition temperature regime was proven by FTIR measurements. If this is the case, it happens after the formation of the seed layer, as nucleation did not seem affected by carbonate contamination. In contrast to the film grown at $520^{\circ} \mathrm{C}$, the polycrystalline film deposited at $565^{\circ} \mathrm{C}$ looks more homogeneous, in spite of some large surface defects. Nevertheless, the electrical characterization of this particular film showed good electrical properties suitable for DRAM application. The roughness of $3.8 \mathrm{~nm}$ is in line with the values of 2.8 and $3.2 \mathrm{~nm}$ observed at the lower thickness of $1.5 \mathrm{~nm}$ and $5 \mathrm{~nm}$, respectively, and indicates a rather continuous growth. 


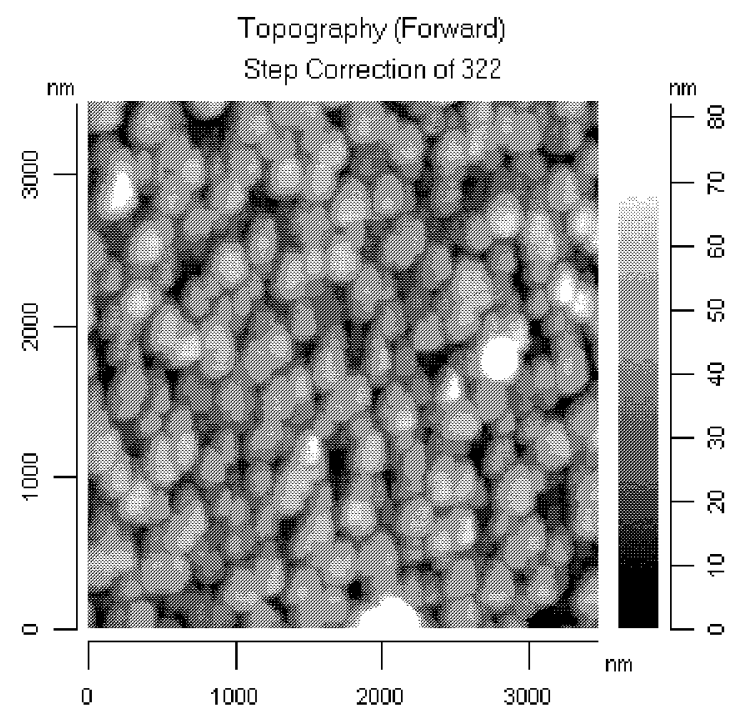

Fig. 6.11: AFM picture of a 30nm film grown at $520^{\circ} \mathrm{C}$. The high roughness $(16.6 \mathrm{~nm})$ results from the random oriented grains.

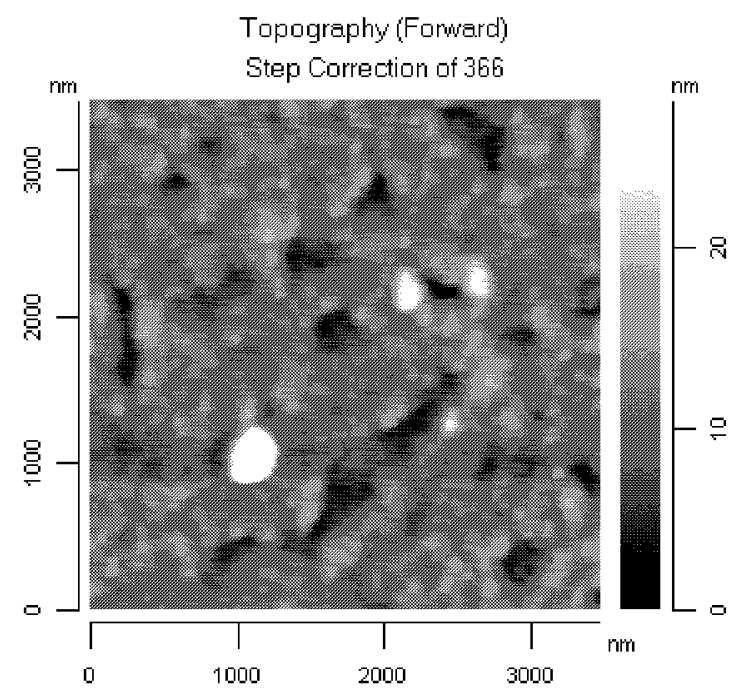

Fig. 6.12: AFM picture of a $30 \mathrm{~nm}$ film grown at $565^{\circ} \mathrm{C}$ with a mean surface roughness of $3.8 \mathrm{~nm}$. Large defects are visible in the scan, but the film still maintains good electrical properties.

The optimum in the surface roughness for growth studies at different deposition temperatures was $2.2 \mathrm{~nm}$ and was reached for the $595^{\circ} \mathrm{C}$ film (Fig. 6.13). For the highest deposition temperature of $655^{\circ} \mathrm{C}$ there is no significant change compared to the film grown at $595^{\circ} \mathrm{C}$ and the roughness is $3.1 \mathrm{~nm}$ (Fig. 6.14). This value agrees well with the roughness observed for the $1.5 \mathrm{~nm}$ film and offers an indication for a continuous growth of the film. Both films seem very flat, however, there is an indication of a change of the modulation wavelength to smaller values at higher temperatures, i.e. from about $100 \mathrm{~nm}$ to about $50 \mathrm{~nm}$.

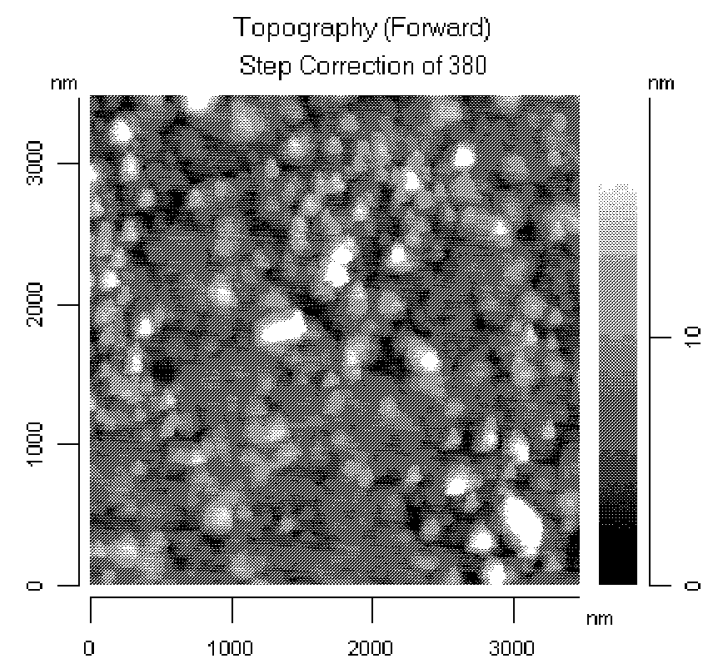

Fig. 6.13: $30 \mathrm{~nm}$ film grown at $595^{\circ} \mathrm{C}$. For this film a mean surface roughness of $2.2 \mathrm{~nm}$ was found.

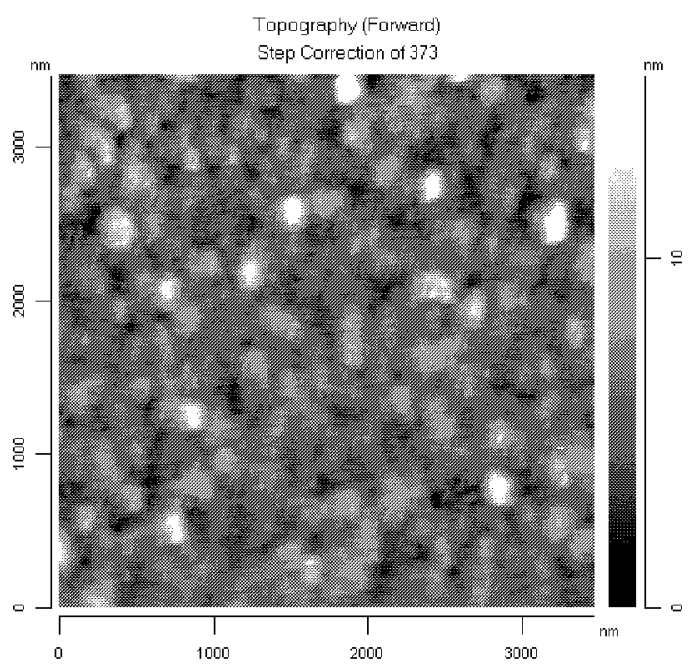

Fig. 6.14: $30 \mathrm{~nm}$ film grown at $655^{\circ} \mathrm{C}$. The roughness of $3.1 \mathrm{~nm}$ is approx. $10 \%$ of the film thickness. 


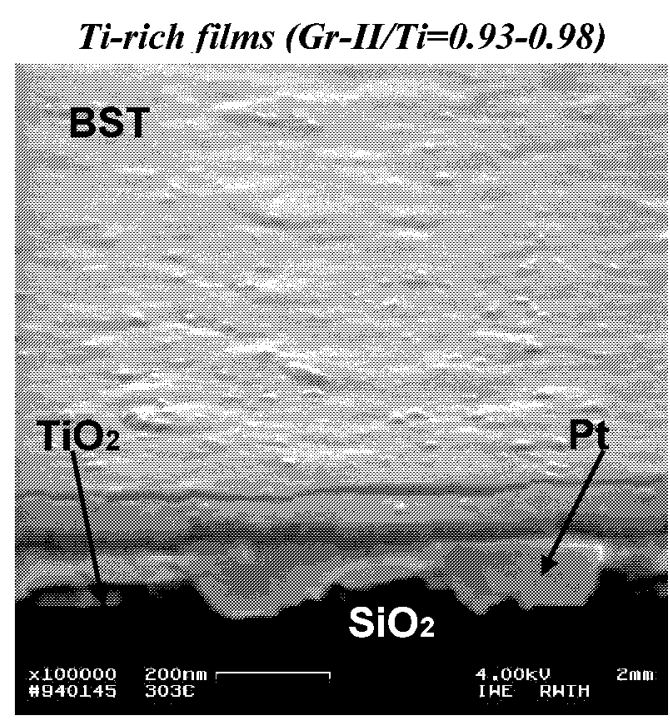

a) $31 \mathrm{~nm}$ film

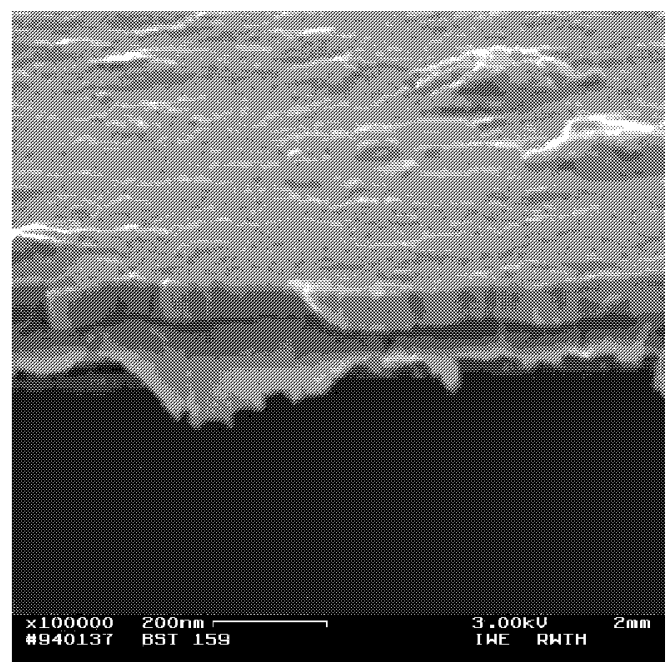

b) $48 \mathrm{~nm}$ film

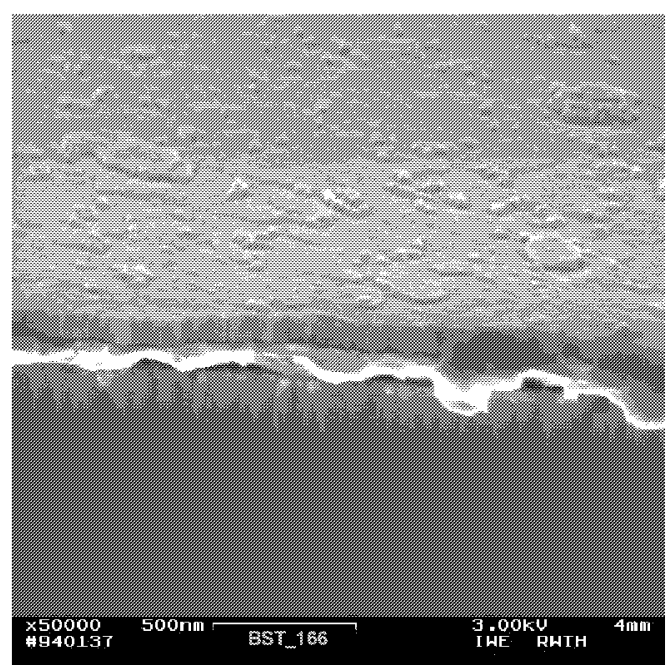

c) $75 \mathrm{~nm}$ film (magnification $\times 50 \mathrm{~K}$ )

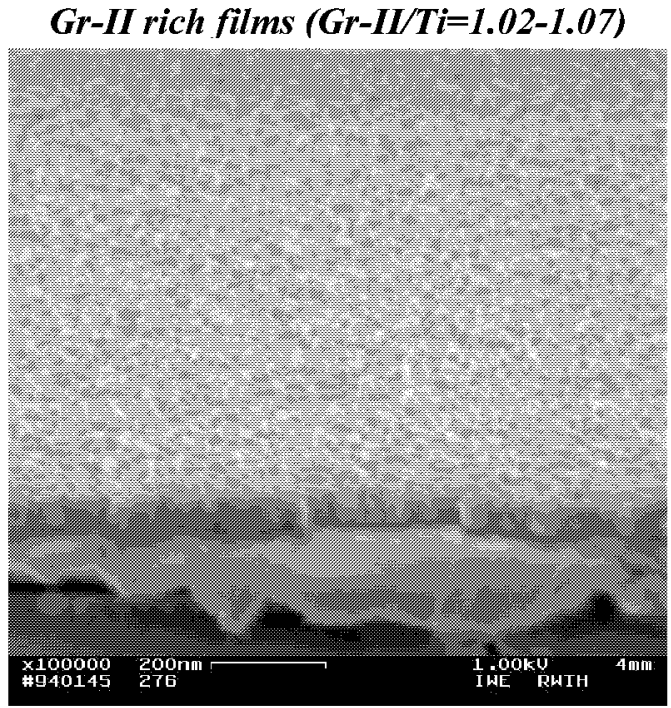

d) $33 \mathrm{~nm}$ film

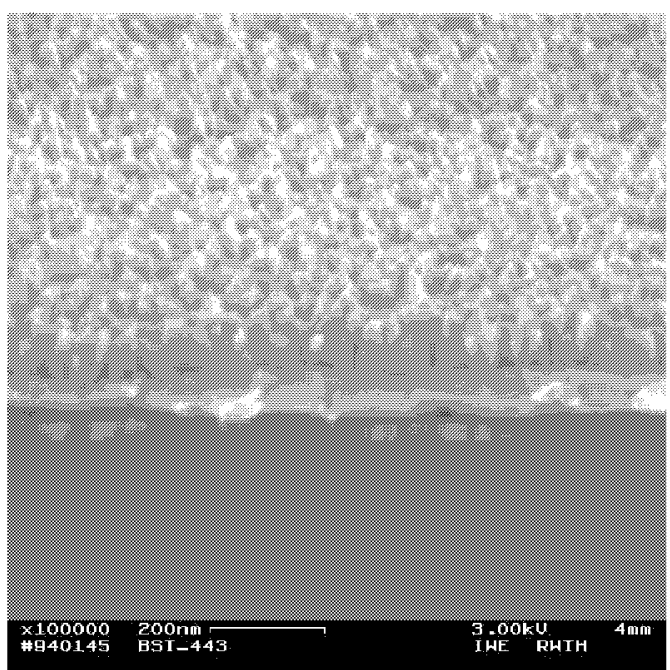

e) $54 \mathrm{~nm}$ film

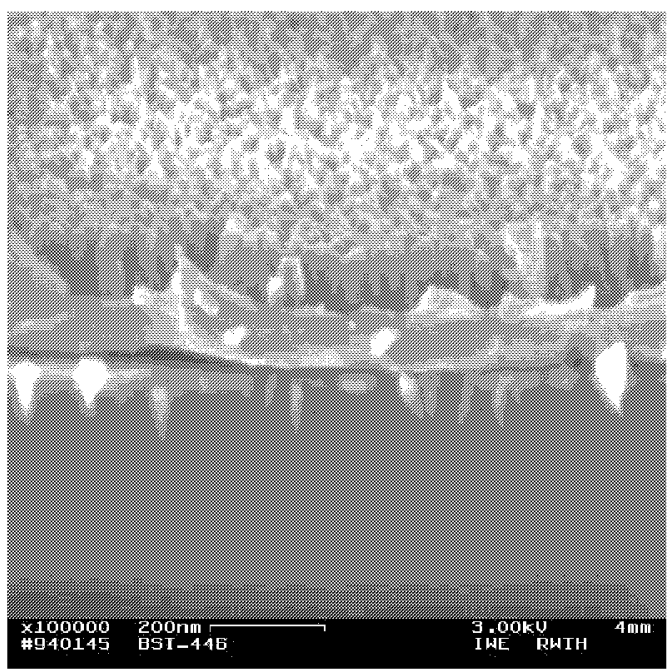

f) $91 \mathrm{~nm}$ film

Fig. 6.15: Thickness series for high- $T_{\text {growth }}$ samples and different stoichiometry regimes 
More details on the grain structure can be obtained from cross-sectional SEM at fracture surfaces. The following micrographs show samples grown at high temperatures $\left(595^{\circ} \mathrm{C}\right.$ and $655^{\circ} \mathrm{C}$ ) and display the influence of the film thickness on the morphology. Additionally, we distinguished between the two basic stoichiometry regimes, e.g. Gr-II and Ti-rich, so that these can be directly compared at different thicknesses (Fig. 6.15). First, a thickness series of Ti-rich films is presented from $31 \mathrm{~nm}$ to $75 \mathrm{~nm}$, placed vertically in the left column, followed by a series of Gr-II samples between $33 \mathrm{~nm}$ and $91 \mathrm{~nm}$. These are vertically positioned in the right column. Generally, high- $T_{\text {growth }}$ results in the formation of strict columnar grains regardless of the total film thickness. The stoichiometry has a significant impact on the film morphology and microstructure, as will be discussed in Section 6.1.3. A careful analysis of the SEM micrographs in Fig. 6.15 shows that the films maintain the same basic microstructure at different thicknesses, as long as the stoichiometry remains constant. Although all SEM samples were prepared in a similar manner, there are often some differences in the electron incidence angle, caused by the manual preparation of the samples, which can lead to a wrong estimation of the film thickness. The thickness values mentioned in the legend are determined using XRF analysis. All figures display the same magnification factor of $\times 100 \mathrm{~K}$ and only the $75 \mathrm{~nm}$ sample on Fig. $6.15 \mathrm{c}$ is magnified by $\times 50 \mathrm{~K}$. We observe a linear behavior between the deposition time and the physical film thickness, that leads to the conclusion that we are in a continuous growth regime independent from any nucleation effects.

\subsubsection{Dependence of the microstructure on the deposition temperature}

For the present discussion we selected $\left(\mathrm{Ba}_{0.7} \mathrm{Sr}_{0.3}\right) \mathrm{TiO}_{3}$ films which are slightly $\mathrm{Ti}$ rich, i.e. $(\mathrm{Gr}-\mathrm{II} / \mathrm{Ti})_{\mathrm{F}}=0.95-0.97$, to discuss the influence of the growth temperature $\left(565^{\circ} \mathrm{C}\right.$ to $\left.655^{\circ} \mathrm{C}\right)$ on the sample crystallinity. We examined the microstructure of films with a thickness around $30 \mathrm{~nm}$, which is close to the envisaged application for DRAMs.

\section{a) Crystallinity and texture}

Fig. 6.16 shows the XRD patterns of films deposited at different temperatures on Pt-substrates with a strong (111)-texture. These are standard diffraction pattern in Bragg-Brentano geometry with a small omega offset in order to suppress the $\mathrm{Si}(\mathrm{h} 00)$ peaks (see Chapter 4.1.1). At $520^{\circ} \mathrm{C}$ the films display very poor crystallinity, not visible in the standard scans and long irradiation times and a thin film grazing incidence geometry are necessary to reveal crystalline peaks. The line pattern of films grown at $565^{\circ} \mathrm{C}$ depicts their polycrystalline structure with a random grain orientation. With rising deposition temperature the (100) orientation becomes preferable and finally we observe a very strong (100)-texture for depositions above $595^{\circ} \mathrm{C}$. Investigations on films with higher thickness displayed the same influence of the growth temperature on their microstructure. The transition from polycrystalline to (100) textured films also seems to influence the surface roughness of the films (also consult the morphology section in 6.1), which increases with lower deposition temperature. The changes in the microstructure of films deposited at different temperatures are strongly related to the different electrical properties of these films, as will be shown in the second part of this chapter. 


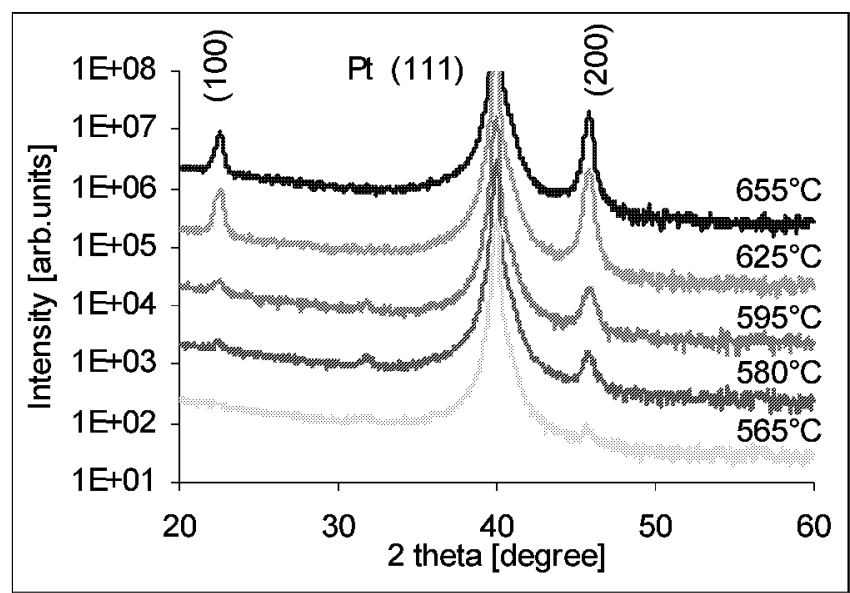

Fig. 6.16: XRD-diagrams for 30nm films deposited at different temperatures on (111) Pt. The films are slightly Ti rich, $(G r-I I / T i)_{F}=0.95-0.97$. The films grown at high temperature $\left(>595^{\circ} \mathrm{C}\right)$ display (100) preferential orientation.

Crystallinity, as represented by the height of the Bragg reflections, also scales with growth temperature.

As revealed by the XRD analysis of the films there is a preferable orientation of the films in the (100) direction for increased deposition temperatures. This strong texture indicates a quasi epitaxial growth on Pt-(111) and is quantified by rocking curves: With the detector set at a known Bragg angle, 2 theta, the sample is rotated through theta. The resulting rocking curve shows the range of grain orientation present in the irradiated area of the film and is a measure of the orientation of the layer. Fig. 6.17 depicts the rocking curves of the (100)-oriented BST film grown at high deposition temperatures $\left(655^{\circ}\right)$, that show a width of only $2-3$ degrees; as this width is similar to that of the underlying $\mathrm{Pt}$, we observe locally a nearly epitaxial growth [107]. This perfectly columnar growth is further documented by the details of the line profiles as shown in Fig. 6.10 for a series of samples grown at $655^{\circ} \mathrm{C}$ : thickness fringes are clearly visible in high resolution scans. Concluding, these results indicate perfectly mono-crystalline (100)-oriented grains and sharp interfaces.

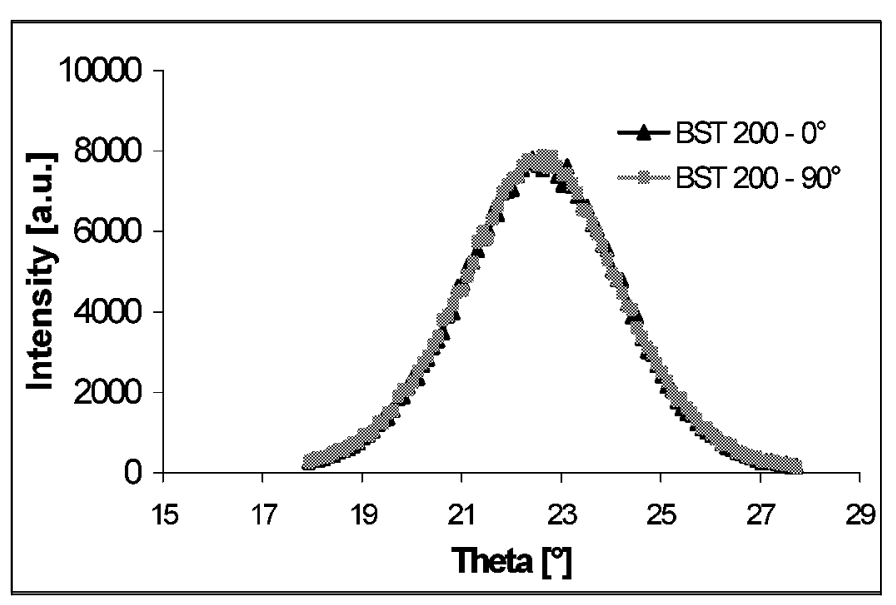

Fig. 6.17: Rocking curves of the (200) reflection for a strongly (100) oriented film on (111) Pt for $\phi=0$ and $\phi=90$. The width of 2-3 degrees shows a locally pseudomorphic growth.

These conclusions are supported by HRTEM pictures (Fig. 6.18), which directly demonstrate this (100)-orientation of the BST on (111)-Pt and the clean and sharp interfaces. The picture gives a closer look at two neighboring grains and details of the boundary region become visible. The crystal structure at both sides of the grain boundary has a slight tilt in the (100) orientation [90]. 


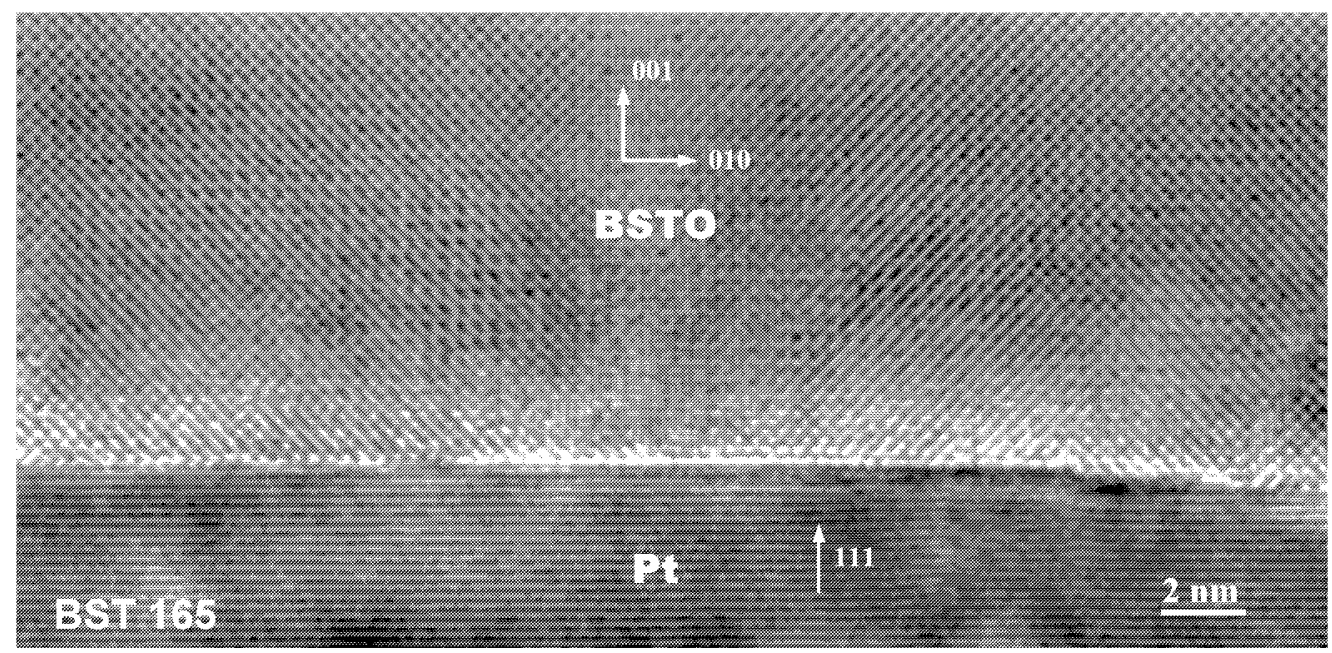

Fig. 6.18: HRTEM micrograph showing the Pt-BST interface for a sample deposited at $655^{\circ} \mathrm{C}$; details of the region around a grain boundary are visible.

\section{b) Strain}

The absolute value of the lattice parameter, as measured normal to the film plane, is smaller than the expected bulk value. This difference results from a tensile stress of the film, which is thermally induced during cooling down due to the different thermal expansion coefficients of BST and of the Si-substrate.

Detailed strain measurements are shown in Fig. 6.19, which yield the expected straight line in a $\sin ^{2} \psi$ plot. The extrapolated value of $\Delta d$ at $\sin ^{2} \psi=1$ corresponds to the tetragonal distortion of the film which can be quantified by the distortion $\varepsilon=\Delta \mathrm{d} / \mathrm{d}$ or by the c/a-ratio $=(\mathrm{d}+\Delta \mathrm{d}) / \mathrm{d}$. Values between -0.6 and $-1.1 \%$ were found for $\varepsilon$, which correspond to values between 0.994 and 0.989 for the c/a- ratio. From this distortion, the film stress $\sigma$ can be estimated, according to the equation:

$$
\sigma=\mathrm{E} /(v+1) \varepsilon
$$

and stresses between 500 and $900 \mathrm{MPa}$ were observed using reasonable values for the elastic constants of BST $(\mathrm{E}=107 \mathrm{GPa}$ and Poisson number, $v=0.3)$. For different films this strain varied between 0.6 and $1.1 \%$, due to variations in the quality of the film and the Pt electrode. Within these variations the expected dependence from the deposition temperature cannot be verified, as there are additional variations due to different film and/or substrate perfection, that cannot be separated.

Most remarkable is the fact that the c/a-ratio is always smaller than 1 and thus opposite to the ferroelectric phase, which is characterized by a larger c-axis. The substrate induced opposite lattice distortion yields therefore a plausible argument for the suppression of the phase transition in these films. 


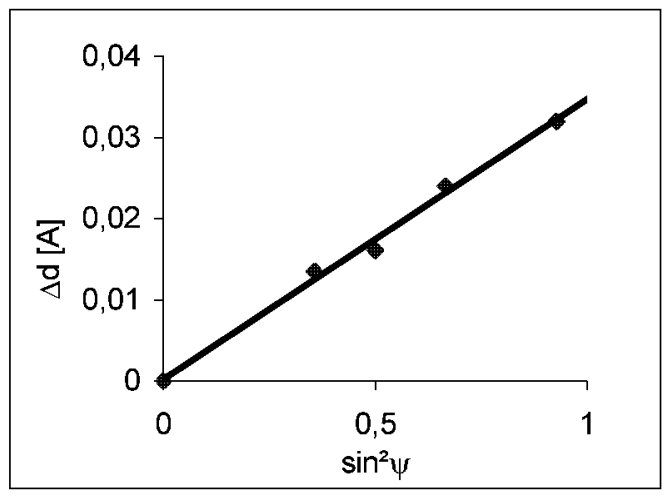

Fig. 6.19: Evaluation of the film strain in a $\sin ^{2} \psi$ plot for a sample grown at $625^{\circ} \mathrm{C}$. Extrapolation for $\sin ^{2} \psi$ equal unity reveals the tetragonal distortion of the film compared to the value of the bulk lattice.

\section{c) Foreign phases}

It is generally of great importance to grow BST films without any contamination from residuals during precursor decomposition or other contamination sources, e.g. particles. Such inhomogeneities among the grains or in the initial phase of nucleation could disturb the seeding growth or interrupt the stable crystal growth resulting into irreproducible film growth. The defects in the films may be caused by single incorporated elements (one or multidimensional defects) but also second phases can be formed.

FTIR yields additional information especially on the formation of foreign phases i.e. carbonates and was used for the investigation of the temperature and stoichiometry dependence of the observation of carbonates. Fig. 6.20 shows FTIR measurements for stoichiometric samples grown at different temperatures $\left(565^{\circ} \mathrm{C}-625^{\circ} \mathrm{C}\right)$. No indication of any carbonate phases can be detected for temperatures above $565^{\circ} \mathrm{C}$ and only the absorption bands which can be assigned to BST are visible around $470 \mathrm{~cm}^{-1}$ and $790 \mathrm{~cm}^{-1}[92-96]$. The small and fine peaks between $1400-1800 \mathrm{~cm}^{-1}$ indicate the presence of $\mathrm{H}_{2} \mathrm{O}$ in the air. Below $580^{\circ} \mathrm{C}$, at $565^{\circ} \mathrm{C}$, FTIR plots reveal the formation of $\mathrm{SrCO}_{3}$ and $\mathrm{BaCO}_{3}$ (sharp peak at $860 \mathrm{~cm}^{-1}$ and broad band around $1450 \mathrm{~cm}^{-1}$ ) and the BST absorption band become broader. Unfortunately, the carbonate peaks could not be separated. Consequently, the term "carbonates" will be referred in the following to both $\mathrm{Sr}$ and $\mathrm{Ba}$ compounds. As the deposition temperature decreases, the BST band become stump, thus indicating that crystallinity becomes worse, which corresponds to the XRD results (also see Fig. 6.16).

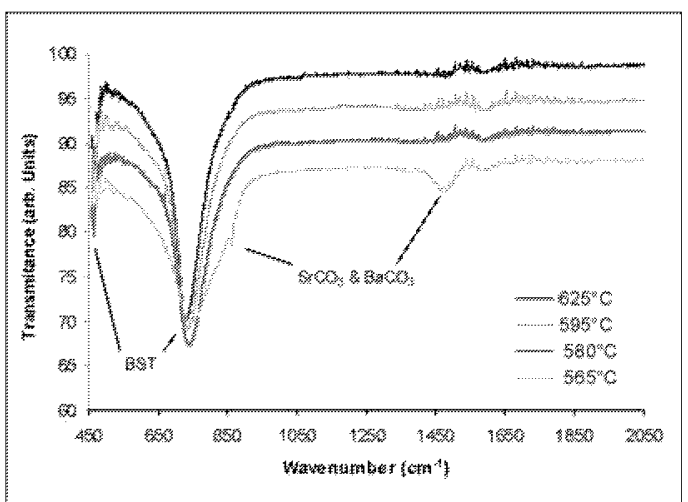

Fig. 6.20: FTIR transmittance plots for stoichiometric samples at different growth temperatures. The carbonate phase is not visible in the films grown beyond $565^{\circ} \mathrm{C}$.

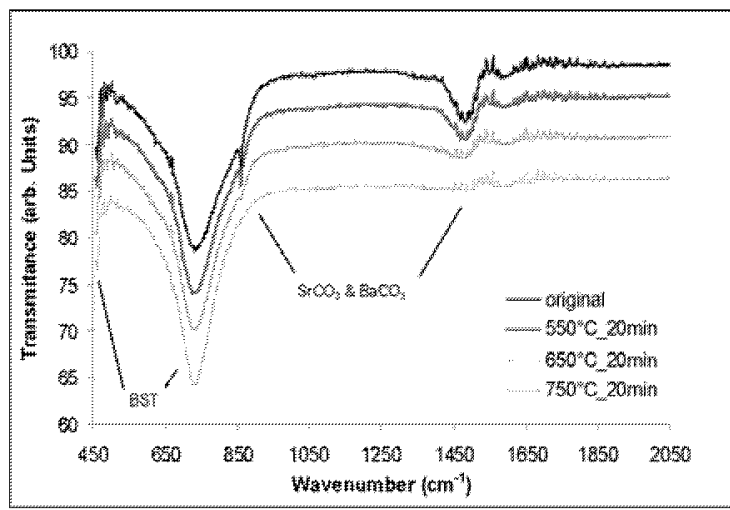

Fig. 6.21: FTIR spectra of a $565^{\circ} \mathrm{C}$ sample after different post-anneal step. The carbonate peaks become lower with increasing annealing steps until they finally disappear after 20min at $750^{\circ} \mathrm{C}$. 
Fig. 6.21 shows a sample which was deposited at $565^{\circ} \mathrm{C}$ and which was annealed for 20 min by rapid thermal annealing (RTA) at four different temperatures from $550^{\circ} \mathrm{C}$ to $750^{\circ} \mathrm{C}$. At elevated temperatures the strong carbonate phase initially becomes weaker and finally disappears after $20 \mathrm{~min}$ at $750^{\circ} \mathrm{C}$. This result is of great importance for the integration of BST in DRAM applications. It was often mentioned that a trade off has to be found between the excellent properties of this material system, if deposited at high temperatures, and the good conformality at lower temperatures. However, the high growth temperature increases the thermal budget and therefore it is possible that a direct crystallization will not meet the requirements of the industry. A step process would be more advantageous and therefore favorable for BST deposition. A detailed investigation of such processes was out of the scope of this work. Nevertheless, some preliminary experiments displayed the feasibility of such processes, although the present deposition tool was not designed for this purpose. Advanced cluster tools are necessary, in order to perform separate single steps in-situ.

XRD was additionally applied to investigate the results of the post annealing procedure. The results of this investigation are presented in Fig. 6.22. This method reveals some weak reflections attributed to the carbonates in the films. These are also present after the $550^{\circ} \mathrm{C}$ step, even though hardly visible. Further, we observe a change in the form of the BST reflections after the high temperature annealing steps. Hence, the peaks appear sharper and well defined, thus displaying a change in the crystal structure. This is reasonable, if we keep in mind that the film was grown at $565^{\circ} \mathrm{C}$, and its exposure to an environment almost $200^{\circ} \mathrm{C}$ hotter makes it highly possible to induce changes in the film, e.g. some grains can grow together, that would explain the difference in the Bragg lines. Even the formation of micro cracks in the surface of the films has been reported after RTA [97]. The direct comparison of the two analytical methods depicts the FTIR as the more useful one for the investigation of carbonate contamination.

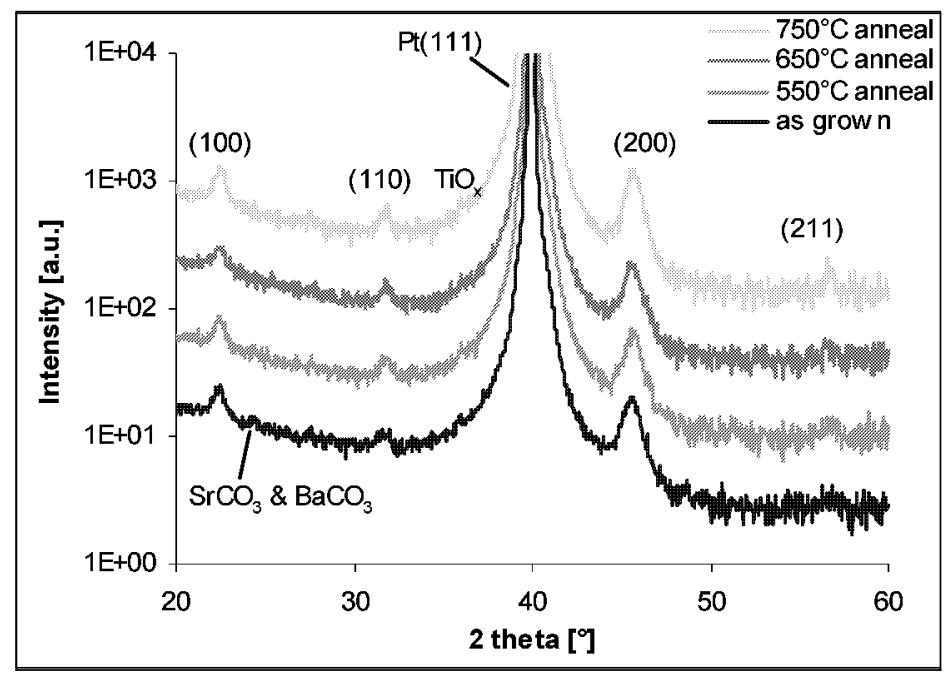

Fig. 6.22: XRD pattern showing second phases (carbonates) in the as-grown film $\left(T_{\text {growt }}=565^{\circ} \mathrm{C}\right)$. The carbonate peaks become weaker with higher post annealing temperatures and they disappear after the $650^{\circ} \mathrm{C}$ step and beyond. The BST lines for the $750^{\circ} \mathrm{C}$ annealing step are sharper compared to the lower temperature steps.

\section{d) Morphology}

In addition to the AFM results discussed above, SEM cross section pictures provide us with information about the grain order in the film and at the interfaces. We chose thicker films to examine the grain structure at different temperatures. The two films in Fig. 6.23 and Fig. 6.24 represent each a low $\left(565^{\circ} \mathrm{C}\right)$ and a high temperature $\left(595^{\circ} \mathrm{C}\right)$ deposition. Indeed we observe a different microstructure, which also correlates to the XRD observations (Fig. 6.16). Perfect columnar grains, uniform (100) oriented, are visible after high temperature growth (see also picture series in Fig. 6.15) and the grains are continuous from the bottom interface to the 
surface. Compared to the high temperature deposition, there is a major difference to the films deposited at the lowest temperature $\left(565^{\circ} \mathrm{C}\right)$. The column growth here seems to be partly interrupted, thus resulting into a more irregular surface, however, the roughness can be compared to Fig. 6.24.

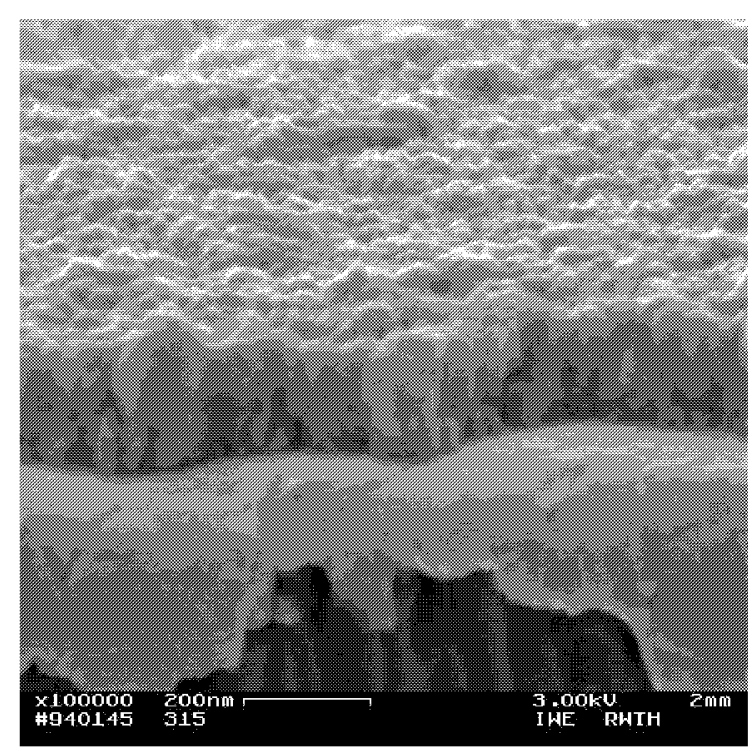

Fig. 6.23: $S E M$ of a $119 \mathrm{~nm}$ film grown at $565^{\circ} \mathrm{C}(\mathrm{Gr}-\mathrm{II} / \mathrm{Ti}=0.92)$ displaying surface and cross-sectional details.

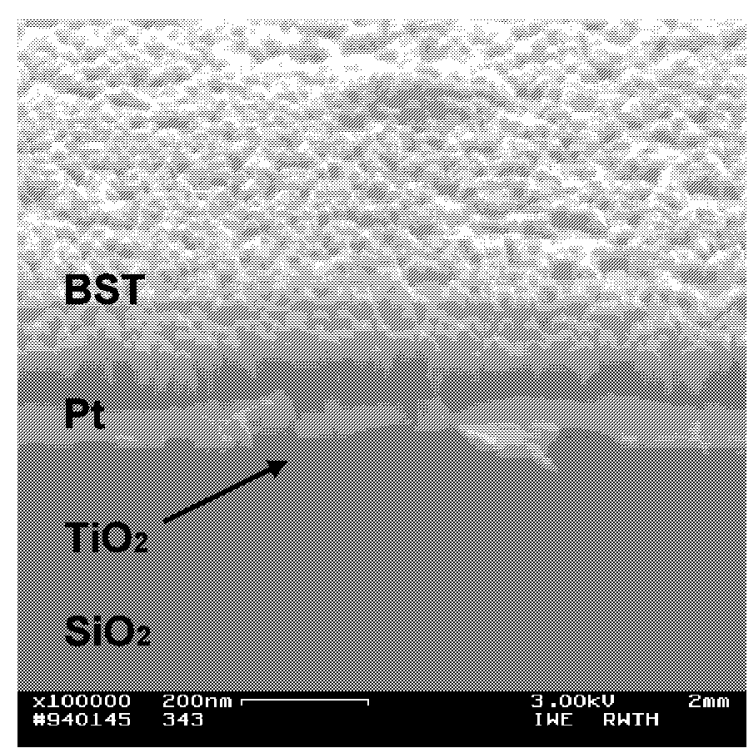

Fig. 6.24: $S E M$ of a $58 \mathrm{~nm}$ film grown at $595^{\circ} \mathrm{C}$ $(G r-I I / T i=0.93)$ displaying surface and crosssectional details.

Next, a more detailed temperature series of Ti-rich films around $30 \mathrm{~nm}$ is presented, in order to evaluate the influence of the growth temperature on the surface roughness. These pictures do not display a significant change in the surface roughness with increasing deposition temperature from $565^{\circ} \mathrm{C}$ (Fig. 6.25) to $655^{\circ} \mathrm{C}$ (Fig. 6.28).

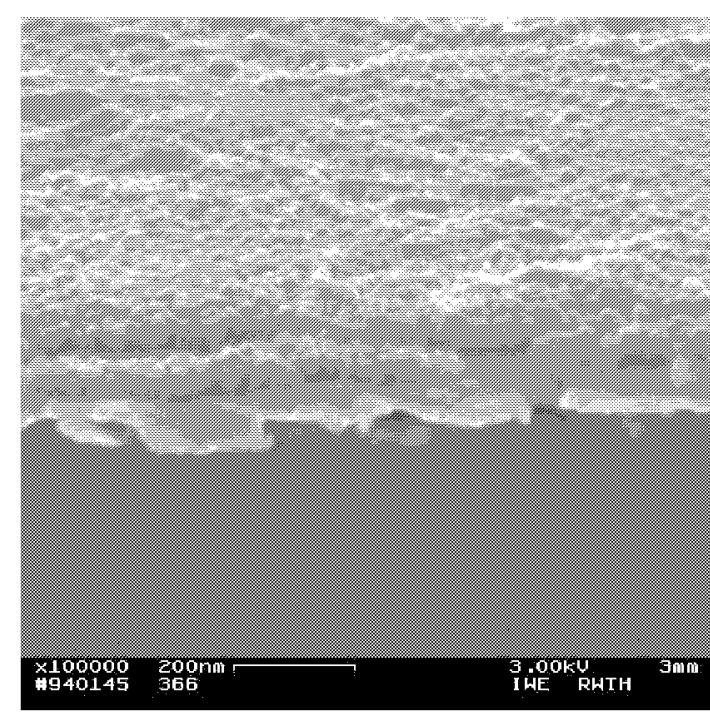

Fig. 6.25: $29 \mathrm{~nm}$ Ti-rich film $(\mathrm{Gr}-\mathrm{II} / \mathrm{Ti}=0.98)$ grown at $565^{\circ} \mathrm{C}$.

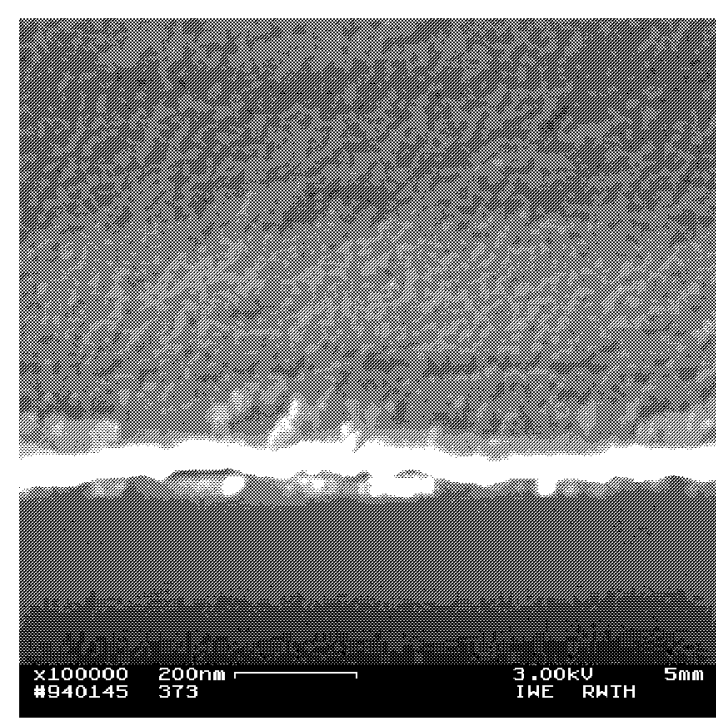

Fig. 6.26: $30 \mathrm{~nm}$ Ti-rich film $(\mathrm{Gr}-\mathrm{II} / \mathrm{Ti}=0.94)$ grown at $595^{\circ} \mathrm{C}$. 


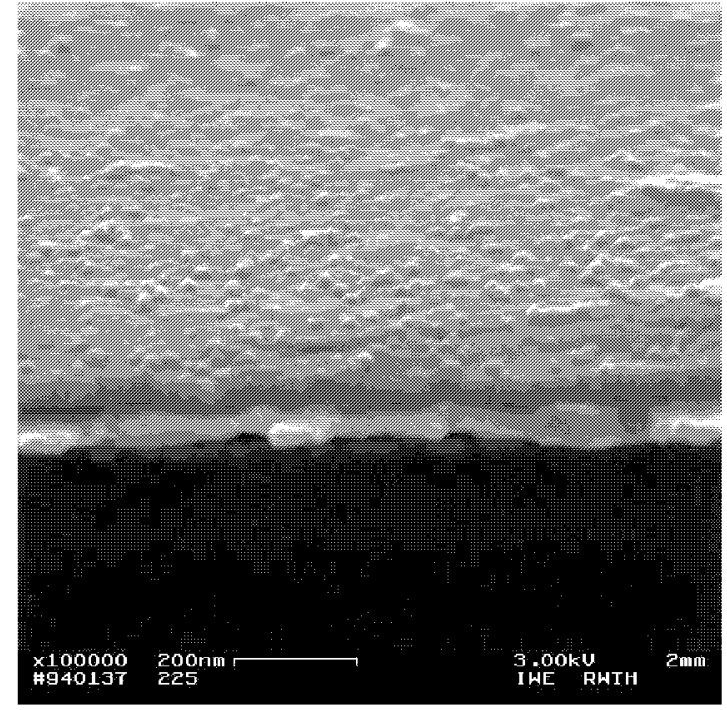

Fig. 6.27: $31 \mathrm{~nm}$ Ti-rich film $(\mathrm{Gr}-I / / T i=0.95)$ grown at $625^{\circ} \mathrm{C}$.

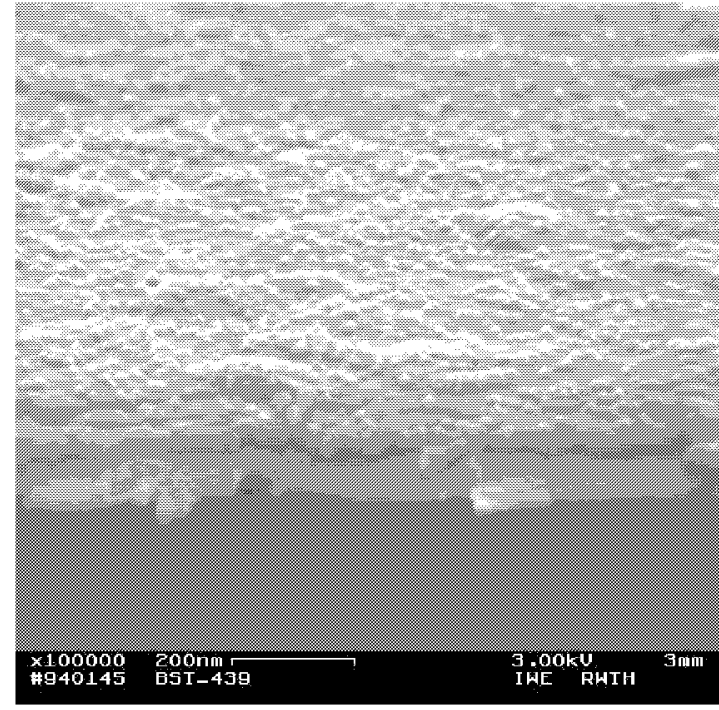

Fig. 6.28: $25 \mathrm{~nm}$ Ti-rich film $(\mathrm{Gr}-\mathrm{IL} / \mathrm{Ti}=0.96)$ grown at $655^{\circ} \mathrm{C}$.

This result is also correlated to the AFM scans. As far as we can conclude from the quality of the pictures, there is a similar growth of the grains despite the different growth temperature, which leads to a similar surface morphology. However, the Ti-rich films generally do not show a homogenous topology in a macroscopical scale. Many explanations for this can be proposed, depending on the incorporation of the Ti excess. Thus, it is possible that additional Ti disturbs the regular grain growth leading to the building of larger crystallites (see Section 6.1.3).

\section{e) Interface properties}

The study of the interface and its role in the nucleation of the films as well as its final electrical properties (see Section 6.2) is of primary interest. Some information about the interface quality was already presented in terms the HRTEM picture that showed a sharp transition between the Pt substrate and the BST film for the high- $\mathrm{T}_{\text {growth }}$ deposition.

SNMS was additionally performed at different deposition temperatures in order to examine any possible interdiffusion of single atoms into the sublayers. All films were grown on standard textured (111) Pt-coated Si substrates with an additional $\mathrm{TiO}_{\mathrm{x}}$ adhesion layer below the $100 \mathrm{~nm}$ Pt film.

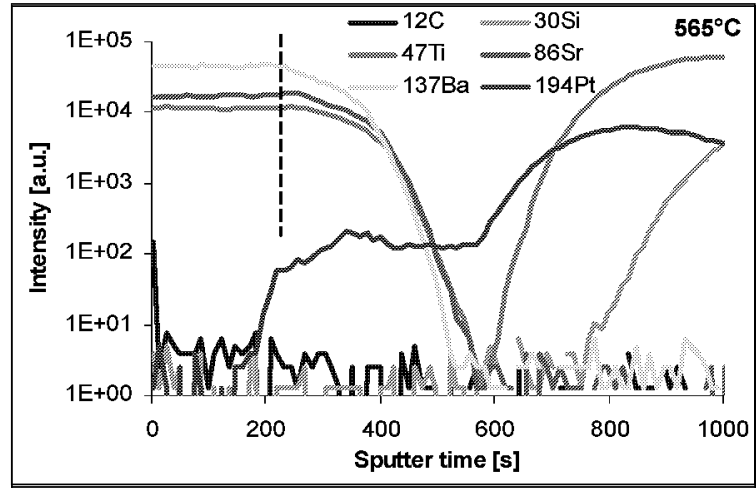

a) Depth profile by SIMS on a stoichiometric $37 \mathrm{~nm}$ BST sample grown at $565^{\circ} \mathrm{C}$. No sharp transition at the BST-Pt interface.

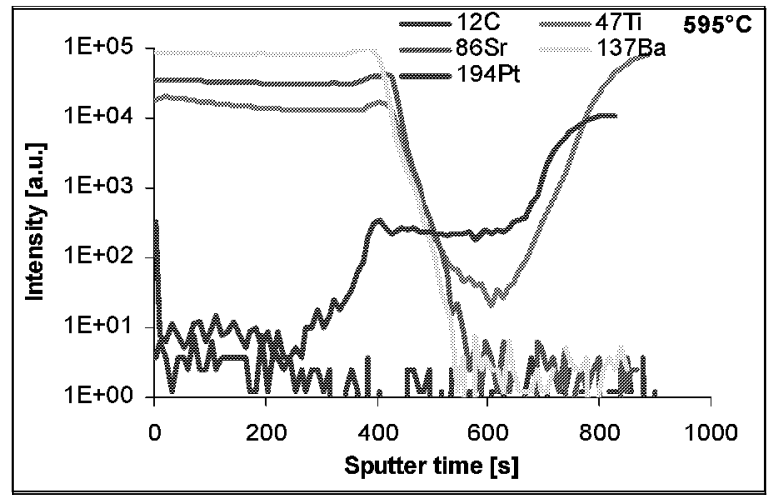

b) SIMS analysis of a $56 \mathrm{~nm}$ stoichiometric sample $\left(T_{\text {growth }}=595^{\circ} \mathrm{C}\right)$. Already at this temperature the interface is well defined possibly due to the low surface roughness. 


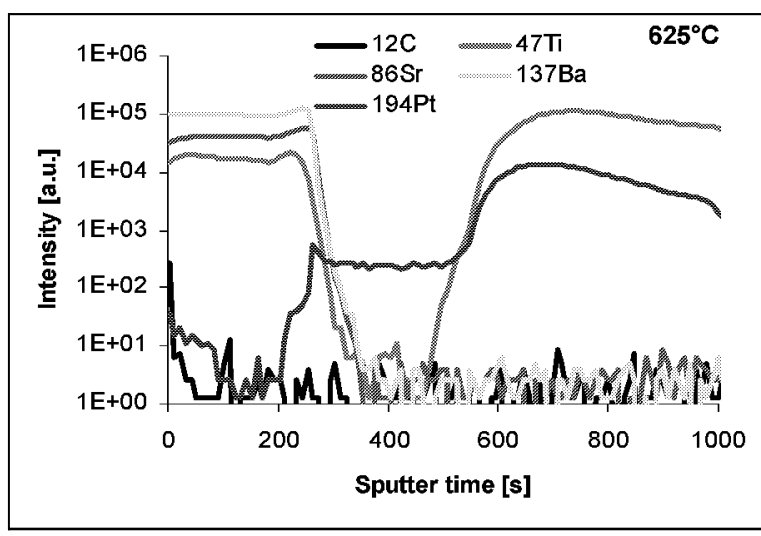

c) SIMS on a $32 \mathrm{~nm}$ stoichiometric sample grown at $625^{\circ} \mathrm{C}$.

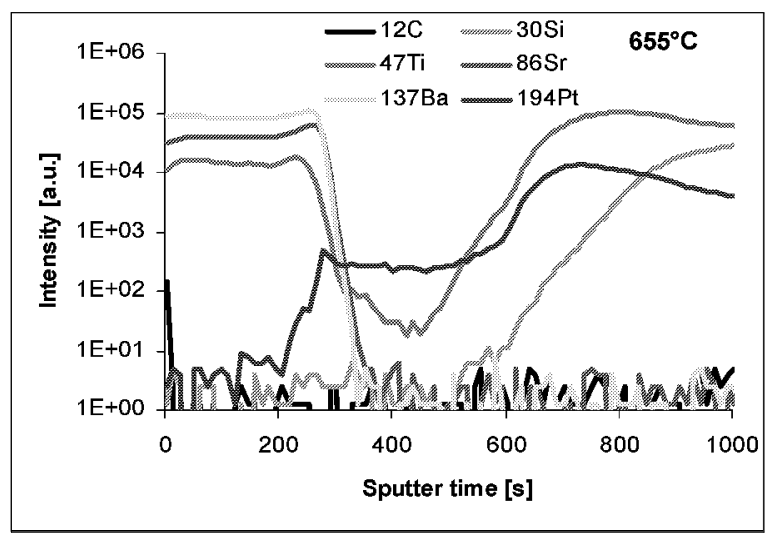

d) SIMS on a $32 \mathrm{~nm}$ slight $\mathrm{Gr}-\mathrm{II}$ rich sample ( $\mathrm{Gr}$ $I I / T i=1.02$ ) grown at $655^{\circ} \mathrm{C}$.

Fig. 6.29: SIMS investigation of the depth profile in a growth temperature series ranging from $565^{\circ} \mathrm{C}$ to $655^{\circ} \mathrm{C}$ in $30^{\circ} \mathrm{C}$ steps.

Typical results are presented in Fig. 6.29a-d for four different growth temperatures. $\mathrm{Cs}^{+}$was used as a primary ion. The following isotopes were used as secondary neutrals: ${ }^{86} \mathrm{Sr},{ }^{137} \mathrm{Ba}$, ${ }^{47} \mathrm{Ti},{ }^{194} \mathrm{Pt}$ and ${ }^{30} \mathrm{Si}$. Additionally, ${ }^{12} \mathrm{C}$ was recorded to monitor the carbon contamination on the surface and in the film. First we take a look at the samples grown at high deposition temperatures $\left(\geq 595^{\circ} \mathrm{C}\right)$. Generally, we obtain a very sharp decrease of the concentration for all elements contained in the film, which is consistent with the sudden increase of the Pt intensity. The suppression of the Pt intensity is a measurement artifact, which occurs at the transition from an oxide to a metallic layer, while using $\mathrm{Cs}^{+}$as a primary ion. Responsible for this is the large difference in the secondary ion yield between the metal and the oxide, that results to a badly defined interface between $\mathrm{Pt}$ and $\mathrm{TiO}_{\mathrm{x}}$. It is possible to avoid this effect by using SNMS analysis with $\mathrm{Ar}^{+}$or $\mathrm{Ga}^{+}$as primary ions. The following increase of the $\mathrm{Ti}$ intensity is attributed to the $\mathrm{Ti}$ adhesion layer. The in-depth element homogeneity is considered good and the small peaks close to the BST-Pt interface indicate a sharp transition. This can be explained through the sudden rise of the sputter rate before the interface, as the ion beam finds a lower atom concentration, while already penetrating the next layer. Also a starting effect is visible, until the sputter process reaches an equilibrium.

On the other hand, there is a major difference compared to the data of the sample grown at a lower temperature $\left(565^{\circ} \mathrm{C}\right.$, see Fig. $\left.6.29 \mathrm{a}\right)$. We observe a good homogeneity of the element concentration in the growth direction. However, the transition to the Pt interface appears very broad, in contrast to the samples processed at higher temperatures. This is attributed to the increased surface roughness of the film, as well as to its polycrystalline structure. The reason for this is the propagation of the surface profile with sputtering until the interface is reached. Thus, part of the ions may have hit the underlying Pt, while some BST rest still remains. Polycrystalline grain support this effect, since the sputter rates may differ significantly for different orientations. Since this method provides us with a local resolution of the depth profile, conclusions generally cannot be made for the total film area. In the previous section, no significant variation in the surface roughness of the $30 \mathrm{~nm}$ films could be determined at different deposition temperatures and, consequently, the observed badly defined transition is not the rule. 


\section{f) Conformal deposition}

We examined the possibility of conformal deposition at different temperatures, since it is a major issue for the integration of BST capacitors. The reduction of the thermal budget is highly desirable, although crystallinity problems arise at low temperatures and a trade off has to be found [86]. We examined MOCVD deposition of BST films on structured platinized wafers at three different deposition temperatures $\left(595^{\circ} \mathrm{C}, 565^{\circ} \mathrm{C}\right.$ and $\left.520^{\circ} \mathrm{C}\right)$. The film microstructure at these temperatures varies from (100)-oriented at $595^{\circ} \mathrm{C}$, polycrystalline at $565^{\circ} \mathrm{C}$ and finally at $520^{\circ} \mathrm{C}$ partly crystalline as revealed by the SEM figures and the weak XRD lines. The conformal deposition of the film was determined from SEM micrographs and is displayed in Fig. 6.30a-c.

Conformality is specified by the step coverage, which is defined according to the following equation as the ratio of the film thickness at the side walls $t_{\text {side }}$ to the thickness at the top of the trench $t_{\text {top }}$ :

$$
C_{\mathrm{SC}}=t_{\text {side }} / t_{\text {top. }}
$$

For the ideal case, we obtain the same film thickness inside the trench and at the surface, which is equivalent with $C_{\mathrm{SC}}$ equal unity. In praxis, however, itis mostly $C_{\mathrm{SC}}<1$. The ratio becomes closer to unity at lower temperatures, that is $C_{\mathrm{SC}}\left(\mathrm{T}_{1}\right)<C_{\mathrm{SC}}\left(\mathrm{T}_{2}\right)$ if $\mathrm{T}_{1}>\mathrm{T}_{2}$. Extensive optimization is necessary for a process with sufficient high step coverage in high aspect ratio trenches to qualify for DRAM application. Due to the lack of proper structured wafers we restricted the necessary runs to a minimum, thus applying our standard growth process without any changes in a short experiment series.

For the $595^{\circ} \mathrm{C}$ sample the step coverage is about 0.55 and becomes 0.66 for the $565^{\circ} \mathrm{C}$ one. Finally, the $520^{\circ} \mathrm{C}$ sample reaches a step coverage of 0.81 . This improvement of the step coverage is observed along with the transition from the transport limited growth to a kinetically controlled growth rate as evidenced by a reduction of the deposition rate below $565^{\circ} \mathrm{C}$. Additionally, crystallinity worsens with lower temperature, so that it seems inevitable to include additional processing steps. Although there are major corrections in thickness necessary due to the non-ideal step coverage and the increased total area, the structured wafers grown at $565^{\circ} \mathrm{C}$ show similar electrical properties in terms of the dielectric constant as the planar films.
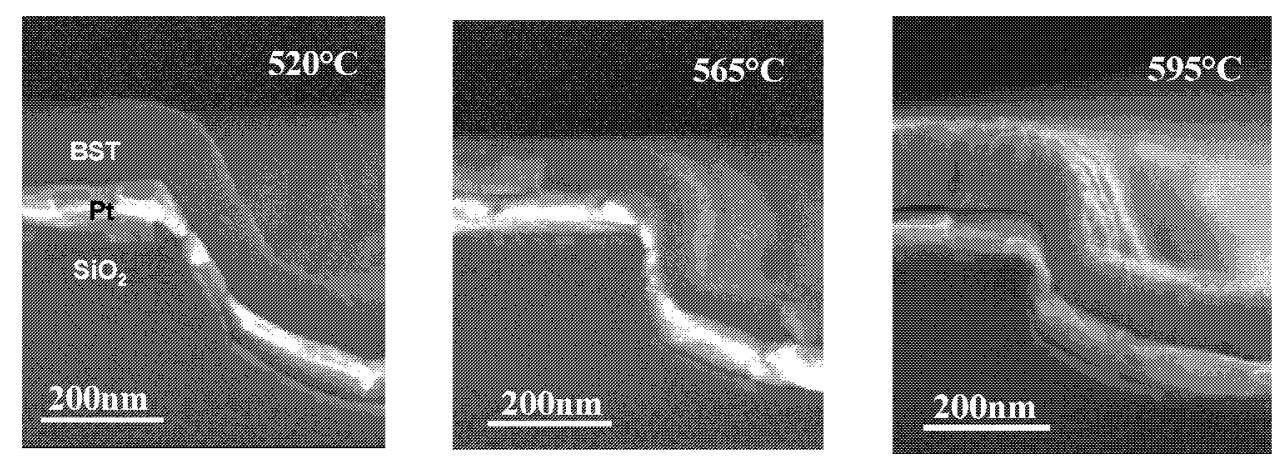

Fig. 6.30: Step coverage for three different susceptor temperatures: a) $520^{\circ} \mathrm{C}$ b) $565^{\circ} \mathrm{C}$ and c) $595^{\circ} \mathrm{C}$, with sample IDs 340,341 and 335 , respectively. The step coverage becomes obviously better with decreasing deposition temperature; 


\subsubsection{Stoichiometry dependence}

\section{a) Variation of the Gr-II/Ti ratio}

The first parameter that was systematically investigated was the film stoichiometry in terms of the Gr-II/Ti ratio, that was varied in the region:

$$
0.85 \leq(G r-I I / T i)_{F} \leq 1.15, \quad \text { Eq. } 6.4
$$

The influence of the film stoichiometry on the microstructure was first studied on films grown at $595^{\circ} \mathrm{C}$, as the film composition altered between $0.94<(\mathrm{Gr}-\mathrm{II} / \mathrm{Ti})_{\mathrm{F}}<1.07$. With increasing Ti-content we observe a trend towards a decrease of the (100)-texture as shown in Fig. 6.31. Additionally, the films turn to polycrystalline below the stoichiometric case, while they are fully (100) oriented in the case of Gr-II excess. Since cristallinity improves with higher GrII/Ti ratio we expect also an improvement of the electrical properties. Fig. 6.32 displays the stoichiometry influence on the crystallinity of $625^{\circ} \mathrm{C}$ grown films. For depositions at $\mathrm{T} \geq$ $625^{\circ} \mathrm{C}$, the dominant $(100)$-growth is stable over a rather wide range of stoichiometries (Gr$\mathrm{II} / \mathrm{Ti}=0.92$ to 1.03 ), and only if the films are far off stoichiometric there is a polycrystalline growth, e.g. the film with $\mathrm{Gr}-\mathrm{II} / \mathrm{Ti}=0.74$ shows poor polycrystalline reflections.
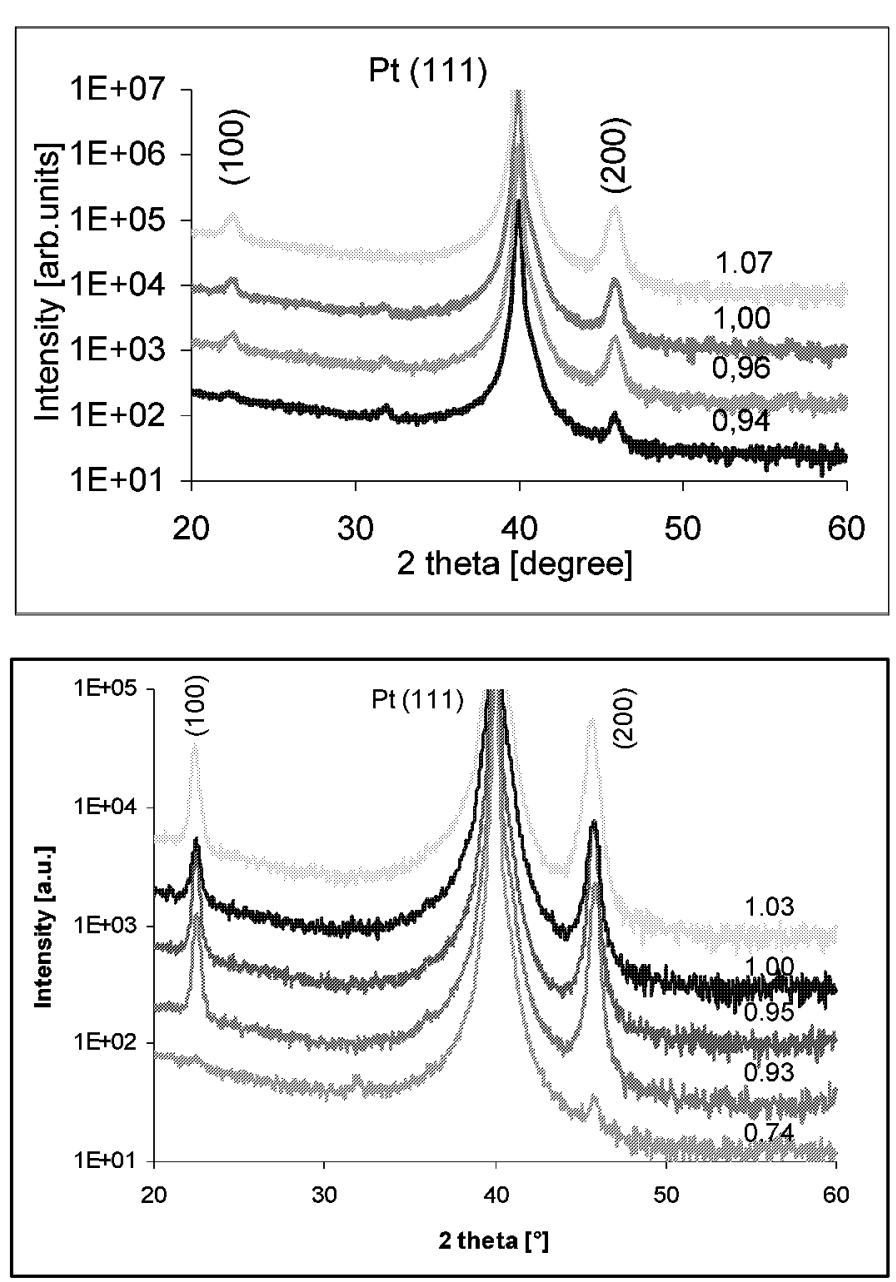

Fig. 6.31: XRD patterns of films around $30 \mathrm{~nm}$ with different stoichiometry in the range of $(G r-I H / T i)_{F}=1.07-0.94$ deposited at $595^{\circ} \mathrm{C}$. The films are polycrystalline as long as they are Ti rich and show stronger (100) texture with higher Gr-II content.

Fig. 6.32: XRD pattern of films around $30 \mathrm{~nm}$ grown at $625^{\circ} \mathrm{C}$ with different stoichiometry ranging from 0.74 to $1.03-(\mathrm{Gr}-\mathrm{II} / \mathrm{Ti})_{\mathrm{F}}-$. The (100) orientation remains stable over a large region.

As shown in the previous graphs, there is an indication for a steady increase of the (100)texture with higher Gr-II content for films grown at lower temperatures as demonstrated in the example of Fig. 6.31. In addition, small changes of the peak-profile as well as of the average lattice parameter, are observed in spite of larger errors due to the weaker and broader peaks. 
Fig. 6.33 summarizes the lattice parameters for films which had a dominant (200)-reflection. Ti-rich samples do not display any changes in the lattice parameter $a$, in contrast to the steady increase with a rising GrII-content as regards to the Gr-II rich samples. Similar observations had been reported for $\mathrm{SrTiO}_{3}$ films and attributed to the incorporation of the excess Gr-II elements in the form of Ruddlesden-Popper phases [98].

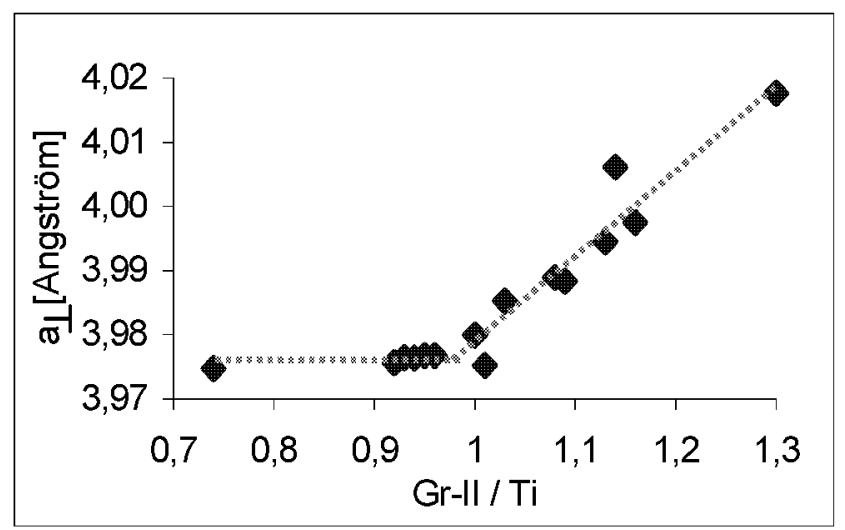

Fig. 6.33: Dependence of the average lattice parameter $a$ on the stoichiometry. The values are taken from a variety of films with a thickness around $30 \mathrm{~nm}$ and a dominant (200) reflection. Despite the significant differences in processing there is an obvious change of the lattice parameter with increasing Gr-II content.

In addition, we observe a change in the peak profile with the variation of the average lattice parameter. There is a broadening and a tail to the low angle side which indicates an inhomogeneous incorporation of the excess group-II elements. Remarkable is the fact, that these structures do not seem very stable, as there is a rearrangement to a more homogeneous structure during annealing in oxygen at a temperature of $550^{\circ} \mathrm{C}$ which is necessary after deposition of the Pt-top-electrodes, Fig. 6.34. For the Ti-rich samples there is no indication of a change of the lattice parameter and also no change in the line profile. Such a behavior is compatible with the formation of stable precipitates of amorphous $\mathrm{TiO}_{\mathrm{x}}$ at the grain boundaries as it has been observed at very high $\mathrm{Ti}$ content and there is no indication for the incorporation of larger amounts of surplus $\mathrm{Ti}$ in the interior of the grains [99].

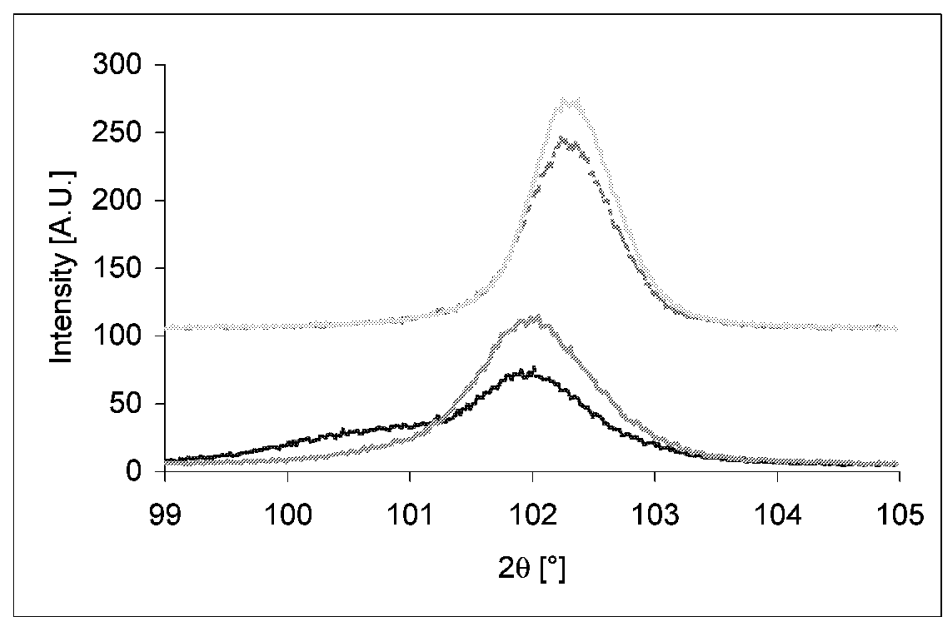

Fig. 6.34: Line shape of the (400)reflection for Ti-rich $($ GrII/Ti=0.93) and GrII-rich (GrII/Ti=1.03) samples $\left(T_{\text {growth }}=625^{\circ} \mathrm{C}\right)$ and change after annealing at $550^{\circ} \mathrm{C}$.

\section{b) Variation of the $\mathrm{Ba} / \mathrm{Sr}$ ratio}

Besides the $(\mathrm{Gr}-\mathrm{II} / \mathrm{Ti})_{\mathrm{F}}$ ratio, we examined the influence of the $(\mathrm{Ba} / \mathrm{Sr})_{\mathrm{F}}$ ratio, which is otherwise fixed around $2.33(\mathrm{Ba} / \mathrm{Sr}=70 / 30)$. For this, three films with different Gr-II ratio were grown at $655^{\circ} \mathrm{C}$. The composition and thickness data of these films were determined by 
$\mathrm{XRF}$ to $(\mathrm{Gr}-\mathrm{II} / \mathrm{Ti})_{\mathrm{F}}=1.00-1.05$ and $23-32 \mathrm{~nm}$, respectively. The $(\mathrm{Ba} / \mathrm{Sr})_{\mathrm{F}}$ ratio was varied from 2.28 to zero (pure STO) and we recorded the XRD plots on (111) Pt as shown in Fig. 6.35.

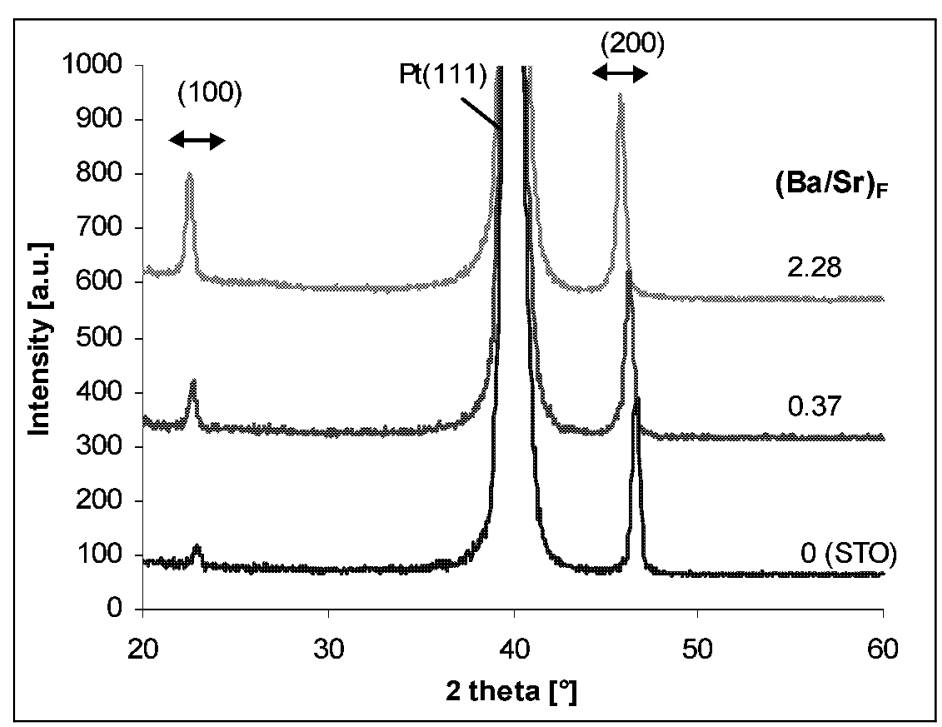

Fig. 6.35: Variation of the $(\mathrm{Ba} / \mathrm{Sr})_{F}$ ratio $(x)$ from $\sim 70 / 30$ to zero. The latter corresponds to pure $\mathrm{SrTiO}_{3}$. We observe a clear shift of the (h00) reflections, more visible being the (200) one, and a scaling of the (100) peak with $x$.

We observe the expected clear shift of the (h00) peak, that is more obvious in the case of the (200) reflexes. Additionally, we observe a decrease of the peak height of the (100) reflection with lower Ba content, which is not expected for the (200) one. Although a (111)-texture of $\mathrm{SrTiO}_{3}$ has been observed for CSD films and a change of the film structure has been reported after MOCVD deposition, we did not observe a change in the texture, i.e. pure $\mathrm{SrTiO}_{3}$ is perfectly (100) textured.

\section{c) Foreign phases}

Fig. 6.36a shows the IR absorption for samples of different stoichiometry at $565^{\circ} \mathrm{C}$. The Gr-II poor sample $(\mathrm{GII} / \mathrm{Ti}=0.93)$ contains no carbonate phases. As the GII content rise, carbonate peaks become visible. Their size is scaling with increasing Gr-II content. Fig. 6.36b shows the stoichiometry dependence for samples deposited at an elevated temperature of $625^{\circ} \mathrm{C}$.

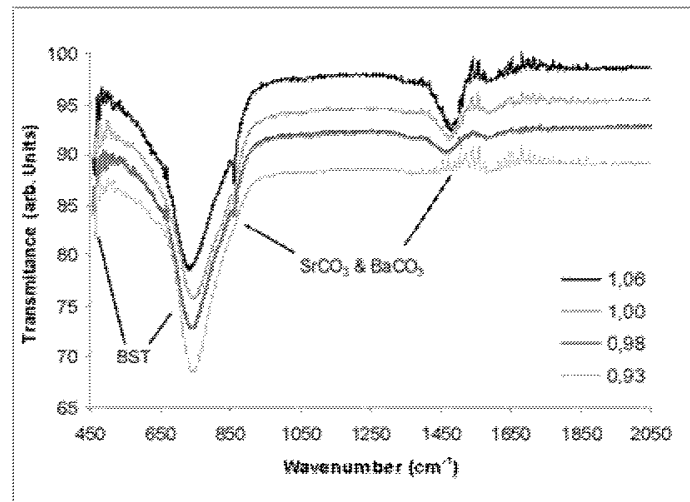

a) FTIR on samples grown at $565^{\circ} \mathrm{C}$ with different stoichiometry between $0.93<(\mathrm{Gr}-I / \mathrm{T} / \mathrm{Ti})_{F}<1.06$.

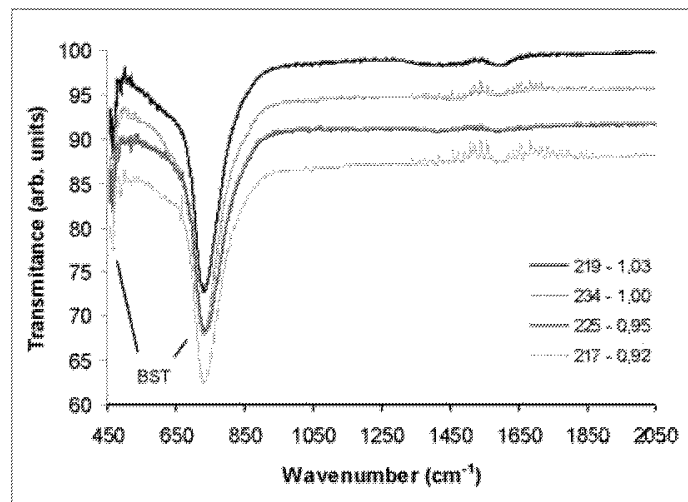

b) FTIR on samples grown at $625^{\circ} \mathrm{C}$ with different stoichiometry between $0.92<(G r-I I / T i)_{F}<1.03$.

Fig. 6.36: Investigation of the carbonate content of BST samples as a function of the stoichiometry at two growth temperatures using FTIR 
At this temperature no carbonate phases are visible at all, despite the changing of the stoichiometry over a wide range. These observations show the need for growing Ti-rich films at low temperature processing. Thus, Ti excess could help to suppress any influence of the carbonates on the thin film growth. On the other hand this is not necessary for high- $\mathrm{T}_{\text {growth }}$ deposition.

\section{d) Film growth and surface roughness}

As already shown in previous SEM pictures, stoichiometry strongly influences the grain growth. Ti excess (see Fig. 6.37) supports the formation of dense films with a locally homogeneous surface and the formation of larger bumps is observed. This may be related to the incorporation of the Ti excess in the grain boundaries. Hence, the surface roughness is increased macroscopically, as pointed to by the arrows.

The mechanism of the grain growth in Gr-II rich films does not obey to the same rule; we obtain a discrete growth of the neighboring grains (see Fig. 6.38) and this grain structure is reflected by the surface roughness. As a result, the roughness is locally enhanced, in contrast to the macroscopical topography, which is very homogenous. AFM measurements support these observations.

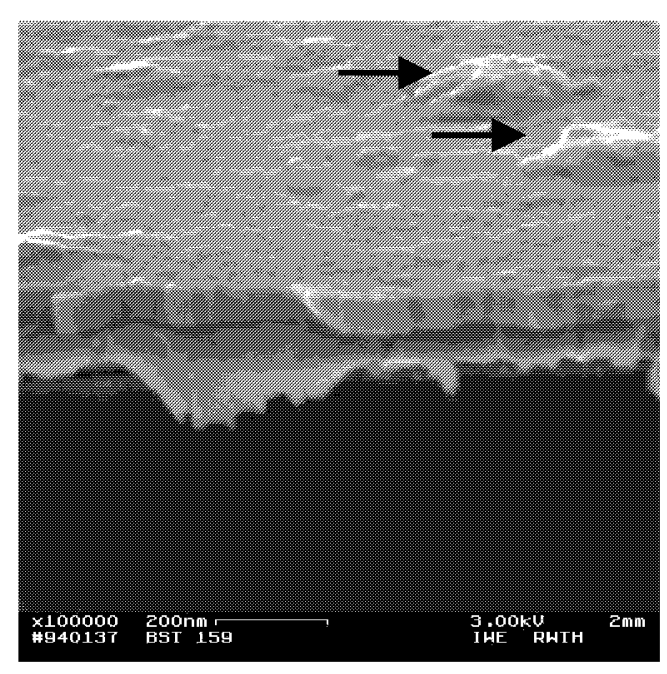

Fig. 6.37: $S E M$ of a $48 \mathrm{~nm}$ Ti-rich (0.93) sample $\left(T_{\text {growth }}=655^{\circ} \mathrm{C}\right)$ showing locally a dense layer; local inhomogeneities appear (arrows).

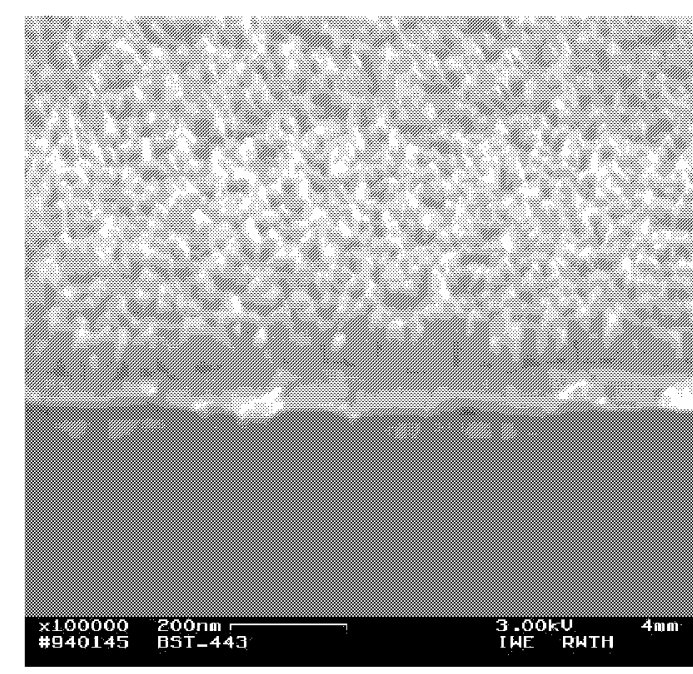

Fig. 6.38: SEM of a $54 \mathrm{~nm}$ Gr-II rich film (1.07) deposited at $655^{\circ} \mathrm{C}$; no humps are visible

The observations were made on films of similar thickness that showed excellent structural and electrical properties, while there was a sufficient margin between the stoichiometry of both films. This different behavior might be explained by a different orientation of the dominant growth facets for different stoichiometry: (111) facets for the Gr-II rich films yield triangular structures and (100) for the Ti rich films yield locally very flat films. Still it is not known whether the humps in the Ti-rich films can be attributed to hillock formation during Pt processing or to inherent film growth mechanisms i.e. a less stable growth. Particle formation seems less likely as these humps are systematically more numerous for $\mathrm{Ti}$ rich films. 


\subsubsection{Influence of the Substrate}

The film growth was investigated on different substrates ranging from amorphous $\mathrm{SiO}_{2}$ on $\mathrm{Si}$, to poorly textured $\mathrm{Pt}$ and to strongly textured $\mathrm{Pt}$ on $\mathrm{TiO}_{\mathrm{x}} / \mathrm{SiO}_{2} / \mathrm{Si}$ or $\mathrm{Zr} / \mathrm{SiO}_{2} / \mathrm{Si}$. Also strongly textured Pt directly on $\mathrm{SiO}_{2} / \mathrm{Si}$, without any adhesion layer was utilized, in order to perform composition analysis via XRF. $\mathrm{SiO}_{2} / \mathrm{Si}$ substrates were widely used in earlier depositions to determine the film stoichiometry and investigate the interdiffusion of the different species into the sublayers. By examining the process data and the film stoichiometry of simultaneously processed $\mathrm{SiO}_{2} / \mathrm{Si}$ and platinized substrates, we observed a significant drift of the film composition which correlates with a difference in the surface temperature between the two substrates. Pyrometer measurements revealed a hotter surface temperature of the platinized wafers relative to the oxidized ones. This difference was in the order of $20^{\circ} \mathrm{C}-50^{\circ} \mathrm{C}$ depending on the specific process conditions (also see reactor performance in Chapter 5).

The most prominent differences between the platinized substrates are the surface roughness and texture and are summarized in Table 6.4. These characteristic values may however vary to some extend for different badges. Fig.6.39 displays an AFM scan of the Pt surface for substrates without adhesion layer supplied by Siemens. The surface roughness of these wafers is similar to the platinized wafers from aixACCT (chapter 6.1.1) and has an rms value of $1.15 \mathrm{~nm}$. Platinized wafers from Radiant were not as good (111) textured as the previously mentioned ones and showed a higher surface roughness of $3.64 \mathrm{~nm}$. The Pt film on these substrates was deposited by magnetron sputtering. Additionally, MOCVD Pt substrates were evaluated, originating from the European HECTOR 300 project. MOCVD Pt in this early development stage has had severe lacks in terms of texture and surface quality.

\begin{tabular}{cccc}
\hline $\begin{array}{c}\text { Substrate } \\
\text { origin }\end{array}$ & Deposition & $\begin{array}{c}\text { Surface } \\
\text { roughness [nm] }\end{array}$ & Orientation \\
\hline AixACCT & sputtering & 1.19 & $(111)$ \\
Siemens & sputtering & 1.15 & $(111)$ \\
& sputtering & 3.64 & $\begin{array}{c}\text { (111) with (200) } \\
\text { component }\end{array}$ \\
Radiant & MOCVD & 18.52 & random \\
\hline
\end{tabular}

Table 6.4 : Quality of different substrate types

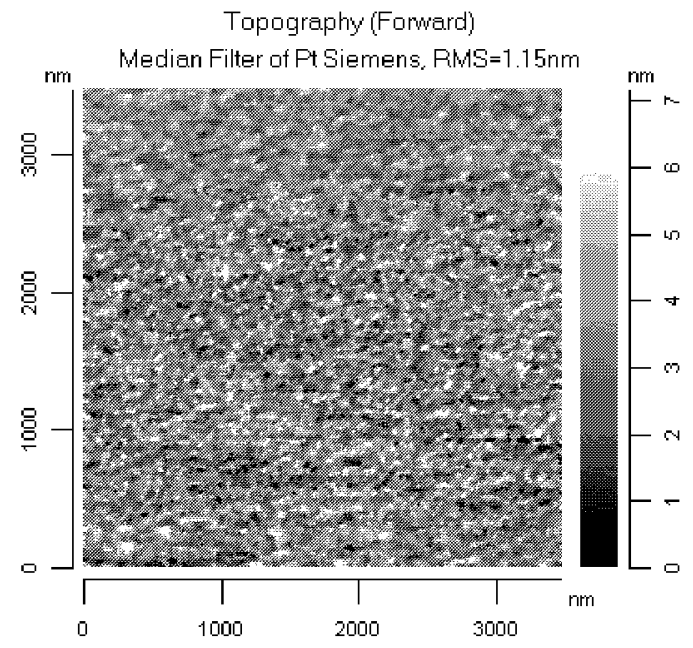

Fig. 6.39: AFM scan of a Pt surface. This substrate quality without Ti adhesion layer for $X R F$ characterization was supplied by Siemens and shows comparable roughness $(1.15 \mathrm{~nm})$ with our standard substrates from aixACCT. 


\section{a) Growth on sputtered Pt}

The planetary reactor is ideal to compare the growth on different substrates, since up to 5 different substrates may be processed simultaneously. The growth on two platinized wafers with different Pt quality and texture is compared in Fig. 6.39. The first wafer with a $\mathrm{Pt} / \mathrm{TiO}_{\mathrm{x}} / \mathrm{SiO}_{2} / \mathrm{Si}$ stack displayed a strong (111) texture, in contrast to the second platinized one with the same stack manufactured by Radiant, which showed a poor texture, e.g. smaller (111) Pt peak and a second (200) Pt line. The growth was performed at $655^{\circ} \mathrm{C}$ and the films were Gr-II rich. Their thickness was determined to $45 \mathrm{~nm}$.

The Bragg BST peaks on perfectly (111)-textured Pt were one order of magnitude higher than the peaks obtained for BST of the same stoichiometry on less perfectly textured Pt electrodes. This intensity decrease is strongly related to the random orientation of substrate and film.

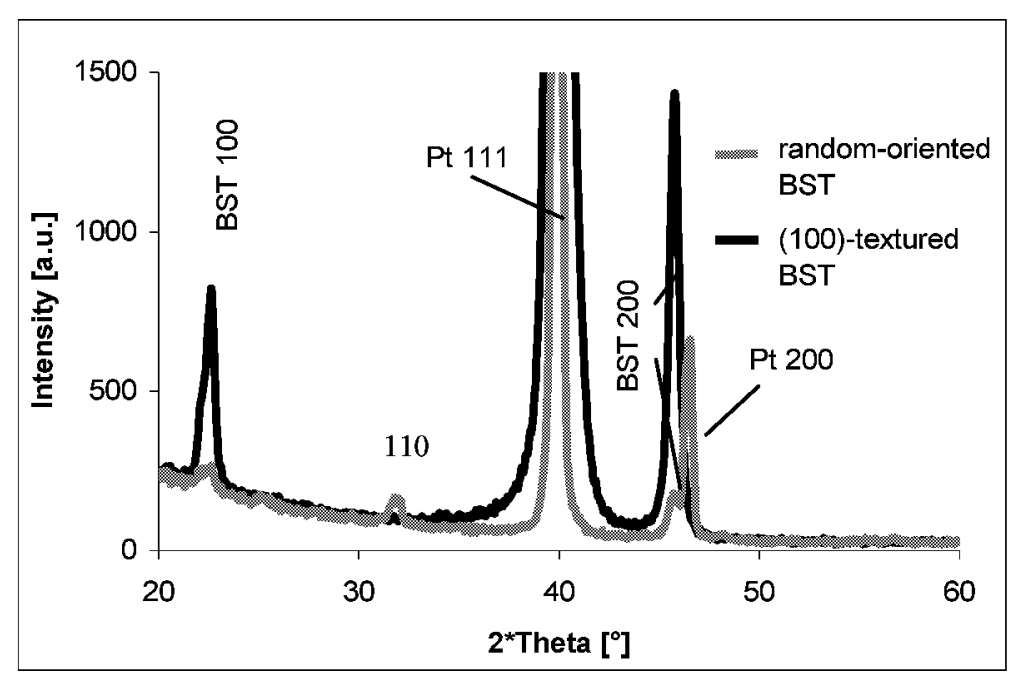

Fig. 6.39: $X R D$ of $B S T$ on Pt. theta-2-theta scan indicating the preferred (100) orientation of film 1; film 2 is nearly random oriented and the Pt (200) reflection is also visible.

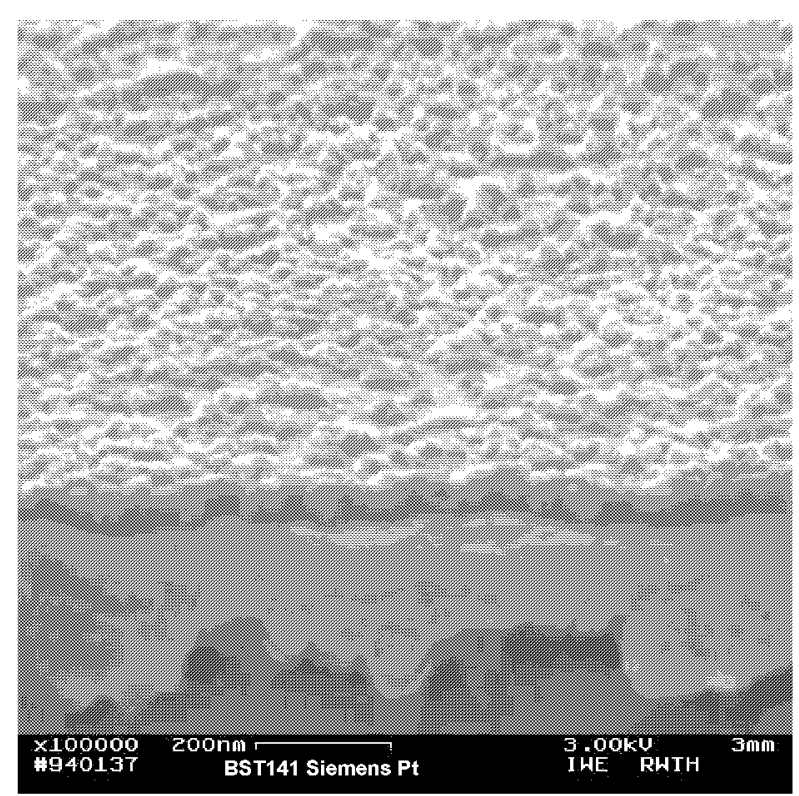

Fig. 6.40: SEM of a $45 \mathrm{~nm}$ BST film on (111) textured Pt (wafer from. The columnar grains display (100) orientation

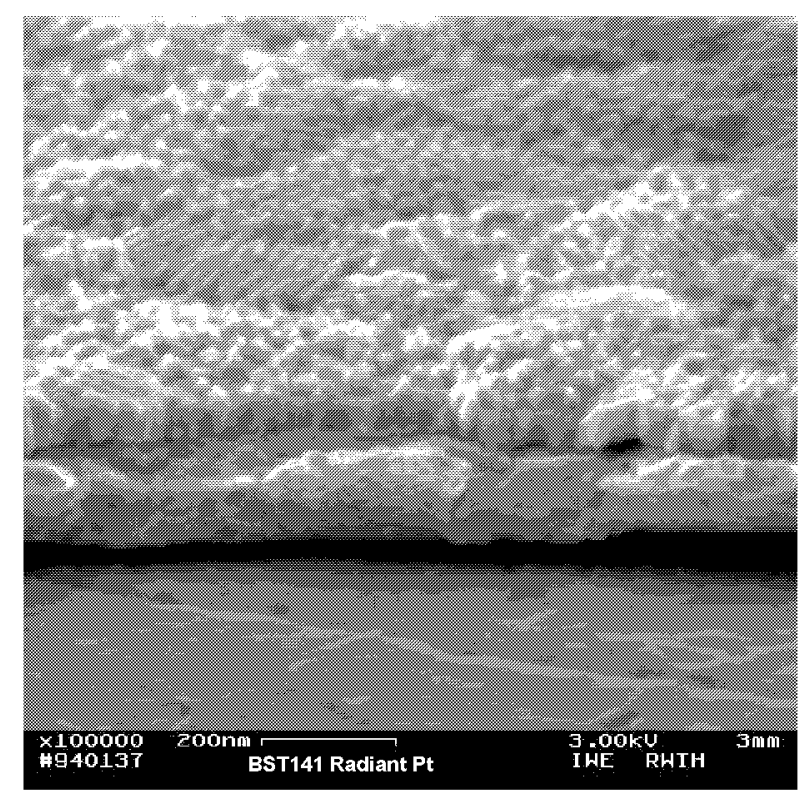

Fig. 6.41: Polycrystalline BST film with $45 \mathrm{~nm}$ thickness on a poor textured Pt surface (wafer from Radiant). Grains are randomly tilted according to the Pt grain orientation. 
The microstructure of the BST-Pt interface was investigated by SEM. Fig. 6.40 shows an example of a columnar grain growth of a (100)-oriented BST film on (111) Pt. The film appears homogenous with no significant surface morphology. The grain size is in the order of $30 \mathrm{~nm}$ for both films. On the weakly textured Pt substrate, Fig. 6.41, the grains remain columnar but are differently tilted, which results in a more rough surface. In the same figure one can also see the influence of the different oriented neighboring Pt grains on the BST texture. As a result film regions of similar dimensions display different orientation. A mean grain size can be approximated to $200-300 \mathrm{~nm}$. Hence, the texture and surface roughness of the Pt bottom electrode has a major impact on the film quality.

Due to the different thermal history there was also different strain in the substrates supplied by aixACCT, which displayed high thermal strain, and by Siemens, which showed small strain. Using the same method described in section 6.1.2, we estimated the strain of different Pt substrates after BST deposition (Fig. 6.42). For our standard Pt coated wafers we obtained an average strain of $0.51 \%$. On the other hand the wafers without $\mathrm{TiO}_{\mathrm{x}}$ adhesion layer that we used for XRF characterization displayed a significant higher average strain value of $0.73 \%$. Remarkable is the fact that the BST films grown on these substrates do not display a similar difference in the strain and we obtain values of $0.84 \%$ and $0.86 \%$ respectively.

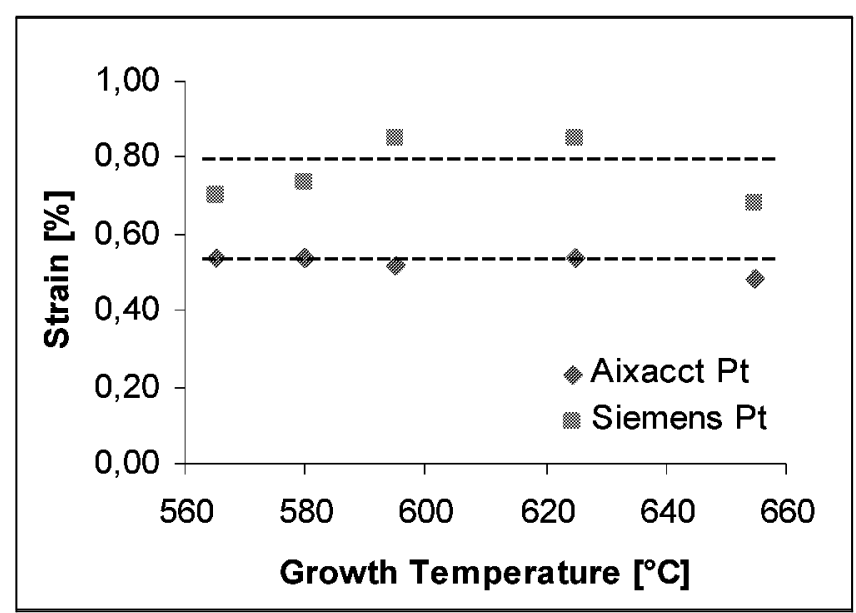

Fig. 6.42: Strain of the Pt substrate as a function of the growth temperature between $565^{\circ} \mathrm{C}$ and $655^{\circ} \mathrm{C}$. The aixACCT Pt displays an almost constant strain in the whole temperature region. The strain of the Siemens Pt is generally higher with a large scattering of the values at different temperatures. Different processing has to be the reason for this behavior.

The aixACCT wafers undergo a post annealing process at $600^{\circ} \mathrm{C}$ after sputtering. Consequently, we did not see a change in the strain with increasing deposition temperature. For the Siemens wafers we could not find any information regarding their processing and their surface roughness was close to this of the aixACCT Pt. Additionally, we observed an obvious increase of the strain after film growth. This behavior is expected for wafers without a post annealing process. Nevertheless, the quality of the Pt in both cases was excellent with only the (111) Pt line depicted in the XRD plots.

SNMS analysis was performed on films grown on different types of substrates in order to determine the in-depth homogeneity of the films and the interdiffusion at the bottom interface. The examined Pt samples (Fig. 6.43: 55nm and Fig. 6.44: 32nm) were grown at $655^{\circ} \mathrm{C}$ and displayed a similar microstructure. An $\mathrm{Ar}^{+}$source accelerated by a voltage of $9.7 \mathrm{kV}$ was used and the mass spectrometer analyzed the isotopes: ${ }^{88} \mathrm{Sr},{ }^{48} \mathrm{Ti},{ }^{138} \mathrm{BST},{ }^{195} \mathrm{Pt}$ and ${ }^{28} \mathrm{Si}$. 


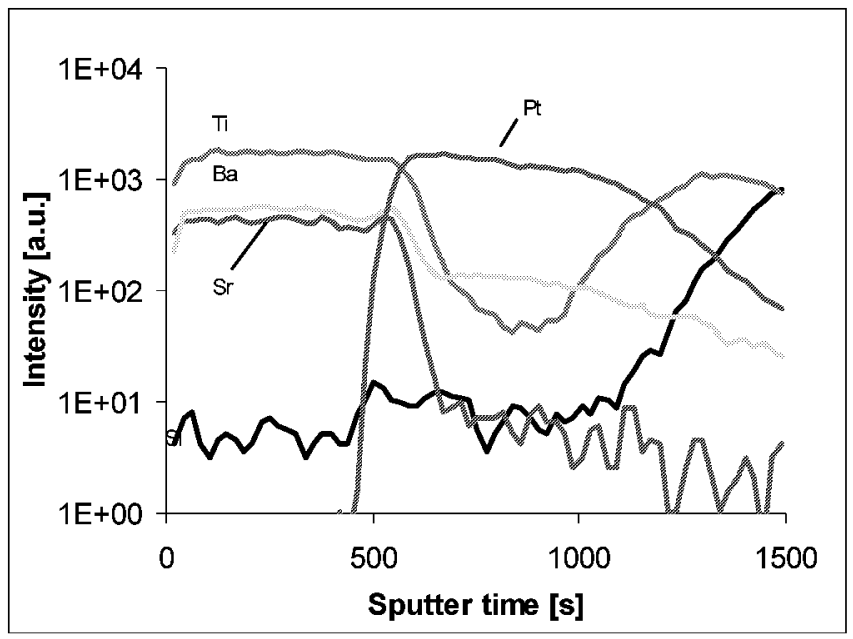

Fig. 6.43: Indication of inter-diffusion at the BST-Pt interface for a $55 \mathrm{~nm}$ sample. This is more evident for $\mathrm{Ba}$; the $\mathrm{Ti}_{x}$ adhesion layer is also visible in the increase of the Ti intensity after the Pt signal falls.

The depth profile is similar for both samples with a good depth profile of the trace elements. There is indication for interdiffusion, especially for $\mathrm{Ba}$ in the case of aixACCT Pt. However, this is not validated by further investigations and could be related to a higher background intensity of the $\mathrm{Ba}$ signal. The $\mathrm{Ti}$ increase at a larger depth in Fig. 6.43 is due to the $\mathrm{TiO}_{2}$ adhesion layer. The analysis of a different prepared Pt coating in Fig. 6.44 shows generally similar trends for the depth homogeneity and only the Ti signal shows a negligible deviation.

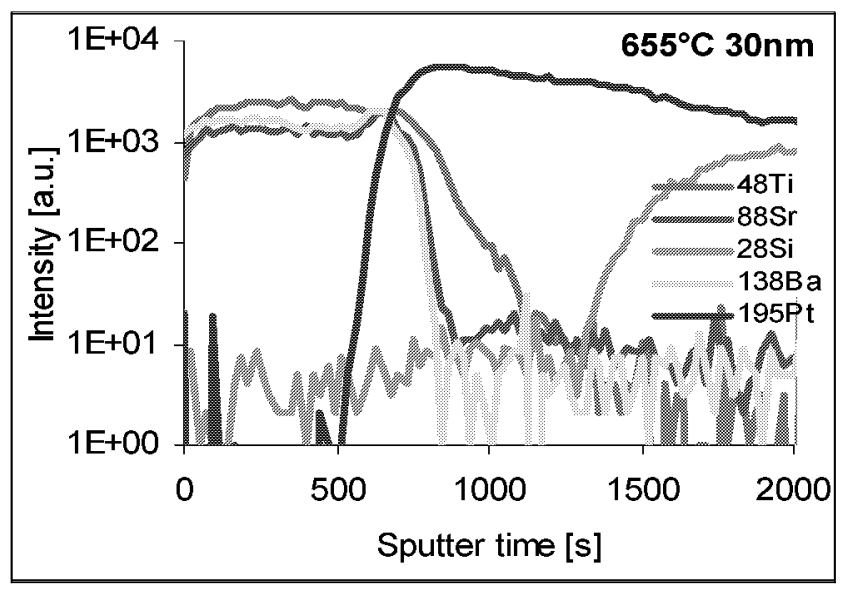

Fig. 6.44: Depth profile of a 30nm BST film on wafers supplied by Siemens. The element homogeneity in the growth direction is comparable to the results on different Pt substrates. The absence of the adhesion layer is visible. The half width of the signal intensity is considered as the end of the respective layer.

\section{b) MOCVD Pt}

MOCVD Pt was deposited on $\mathrm{TiO}_{\mathbf{x}} / \mathrm{SiO}_{2} / \mathrm{Si}$ substrates within the HECTOR300 project and Ti-rich BST films of $49-97 \mathrm{~nm}$ thickness were deposited using our standard growth process at $595^{\circ} \mathrm{C}$. The XRD patterns plotted in Fig. 6.45 reveals a polycrystalline characteristic. The plots show a clear scaling of the peak height with thickness and both the (111) and the (200) Pt lines. As a comparison, a $71 \mathrm{~nm}$ film on our standard sputtered (111) Pt is displayed. 


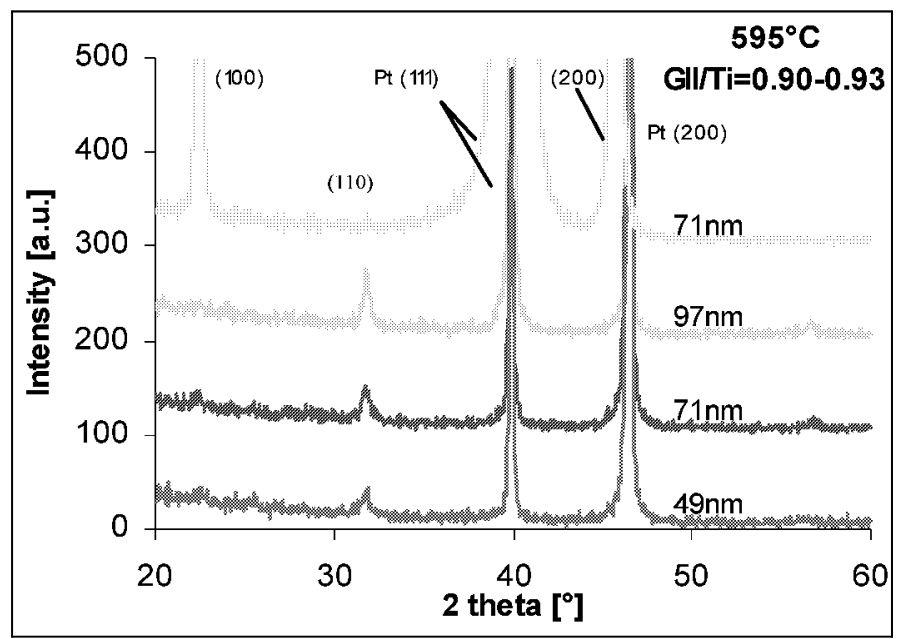

Fig. 6.45: $X R D$ scans of Ti-rich films with different thickness (49-97nm). The growth was performed on MOCVD Pt substrates. A simultaneously processed $71 \mathrm{~nm}$ film on (111) Pt is also depicted.

Hence the texture of the Pt-film is comparable to the RADIANT films but the roughness is much higher. The SEM pictures in Fig. 6.46 display the morphology of two samples with different thickness and their high roughness, which is in the order of $18 \mathrm{~nm}$. In addition, many $100 \mathrm{~nm}$ local peaks are visible that seem to be single grains or larger crystallites. The topology of the surface is similar for both thicknesses and the grain growth seems frustrated, although the deposition temperature is high. The roughness appears quite high compared to the films on highly (111) textured $\mathrm{Pt}$, and was measured to be $\sim 16.4 \mathrm{~nm}$. This high roughness also leads to electric shortcuts after deposition of the top electrode for films below $30 \mathrm{~nm}$ thickness.

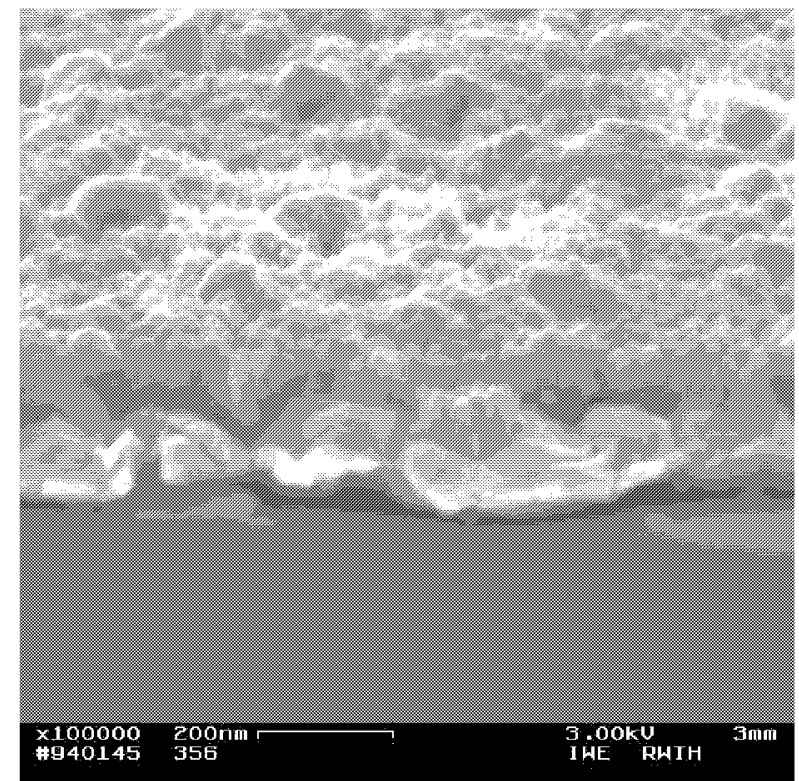

a) 49nm Ti-rich BST film ( $\mathrm{Gr}-\mathrm{II} / \mathrm{Ti}=0.92)$

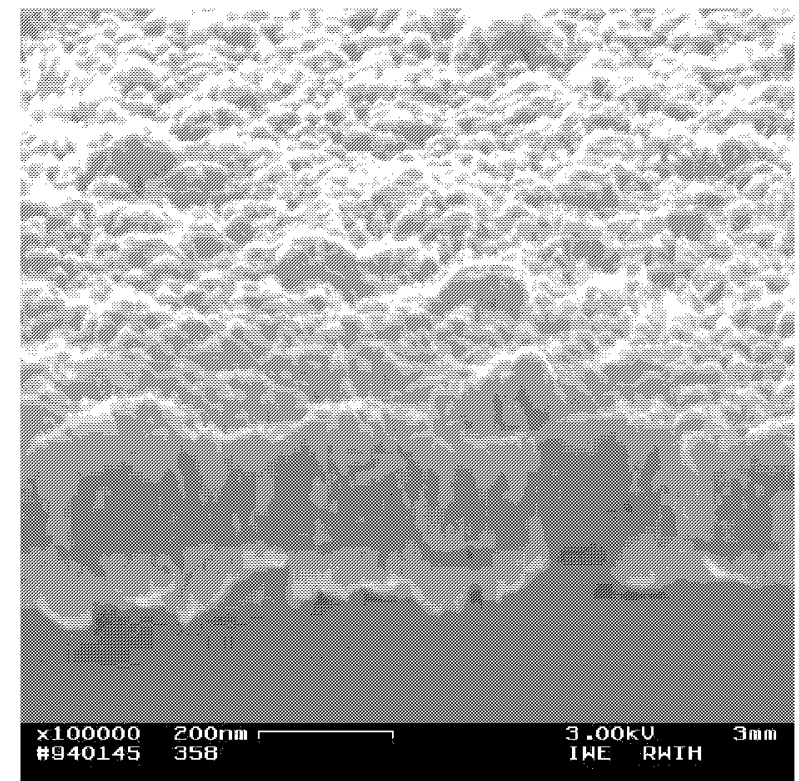

b) $97 \mathrm{~nm}$ Ti-rich BST film (Gr-II/Ti=0.93)

Fig. 6.46: SEM figures of BST films grown on MOCVD Pt during the HECTOR300 project.

\section{c) Growth on $\mathrm{SiO}_{2}$}

Fig. 6.47 displays the XRD plots of BST films grown at $655^{\circ} \mathrm{C}$ on $\mathrm{SiO}_{2} / \mathrm{Si}$ wafers. The legend shows the $(\mathrm{Ba}+\mathrm{Sr} / \mathrm{Ti})_{\mathrm{F}}$ ratio of the films together with the films' thickness. For temperatures above $600^{\circ} \mathrm{C}$ we generally obtained good crystalline growth for Ti-rich films on $\mathrm{SiO}_{2} / \mathrm{Si}$ 
substrates over a very broad range of stoichiometry. For $(\mathrm{Ba}+\mathrm{Sr} / \mathrm{Ti})_{\mathrm{F}}=0.32$ we obtain amorphous growth, that turns into polycrystalline beyond 0.50 . As the films became $\mathrm{Ba}$ - and Sr-rich they were more randomly oriented and second phase formation, $\mathrm{BaCO}_{3} / \mathrm{SrCO}_{3}$, at $(\mathrm{Ba}+\mathrm{Sr} / \mathrm{Ti})_{\mathrm{F}}=1.11$ could be observed.
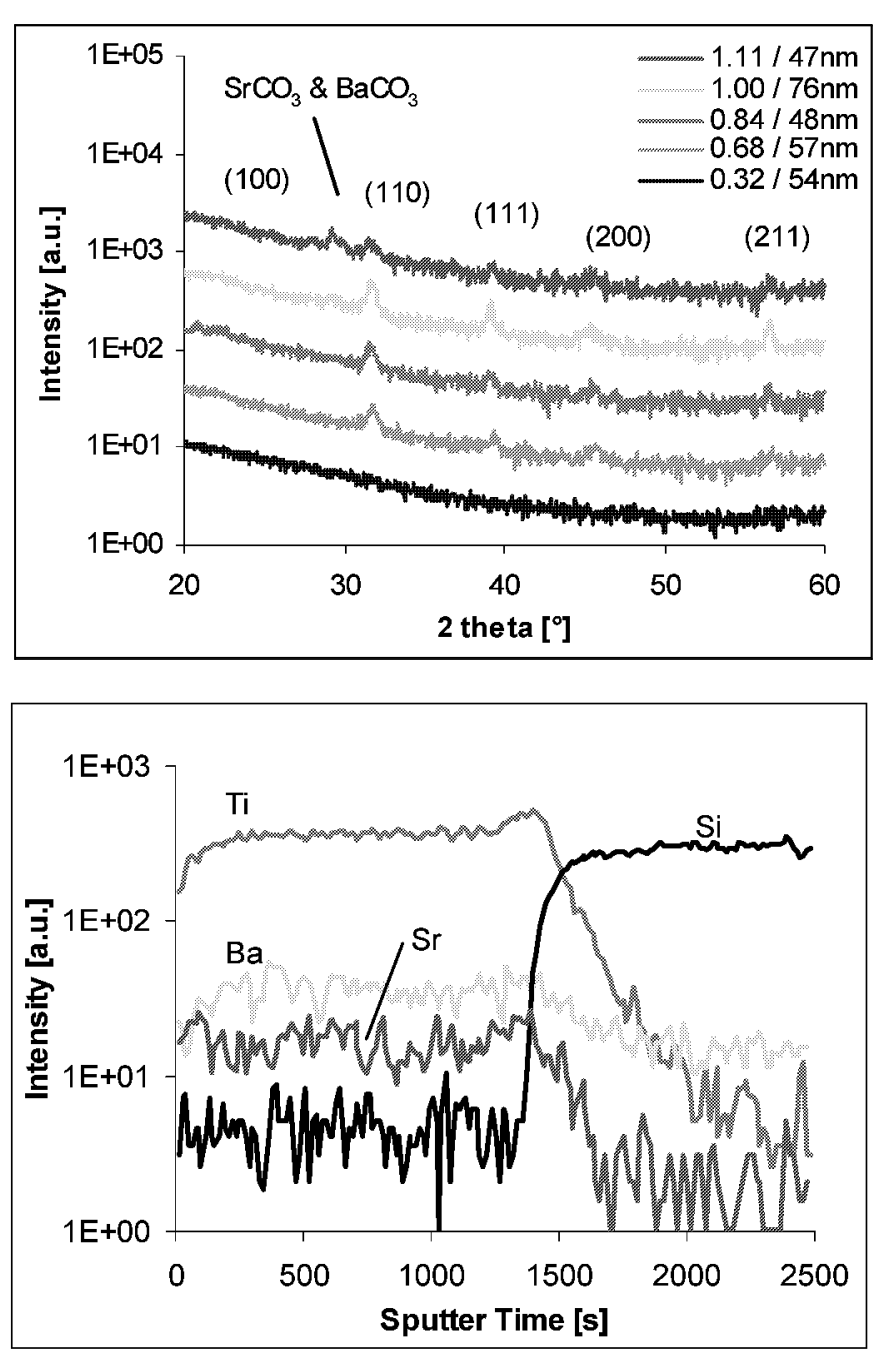

Fig. 6.47: Diffraction pattern of BST on $\mathrm{SiO}_{2} / \mathrm{Si}$ substrates. Polycrystalline growth is observed across a large stoichiometry regime. Gr-II rich films maintain the polycrystalline character and growth of carbonate phases is visible. The legend presents first the stoichiometry of the BST film, $(G r-I I / T i)_{F}$, followed by the film thickness.

Fig. 6.48: SNMS spectrum of a BST $/ \mathrm{SiO}_{2} / \mathrm{Si}$ stack showing the depth profile of a film with a thickness of $55 \mathrm{~nm}$. Good indepth homogeneity is observed; $B a$ interdiffusion into the $\mathrm{SiO}_{2}$ sublayer is visible.

Fig. 6.48 shows a good in-depth deposition homogeneity and indicates that all elements diffuse into the $\mathrm{SiO}_{2}$ layer, $\mathrm{Ba}$ much stronger than $\mathrm{Ti}$, since the transition is not as sharp as for the deposition on Pt substrates. Again we observe a rather high background intensity for the $\mathrm{Ba}$ signal. The rise of the intensity at the interfaces between $\mathrm{BST} / \mathrm{SiO}_{2}$ and $\mathrm{BST} / \mathrm{Pt}$ is a measurement artifact and happens due to the sudden rising of the sputter rate during the transition to a different element matrix. At the beginning of the analysis, we also observe a typical starting effect expressed in the slow rise of the sputter gain, mainly due to absorption from surface residues. Depending on the energy and the type of the primary ions an equilibrium is usually reached after a few $\mathrm{nm}$ (here $\sim 5 \mathrm{~nm}$ ). 


\subsection{Electrical properties of BST capacitors}

For the electrical characterization Pt top electrodes were deposited by magnetron sputtering and structured using a lithographic lift-off technique. The diameter of the electrodes varied from 0.1 to $1.13 \mathrm{~mm}$. An additional post-annealing was performed ex-situ in order to repair possible damage from the sputtering. The annealing temperature was always below the growth temperature of the films and variations of the standard annealing process yielded small variations in the value of the effective permittivity of our films. However, we could not determine any significant dependency on the annealing parameters. The permittivity values presented here are measured after the post annealing step.

The standard electrical characterization included the dielectric permittivity $\left(\varepsilon_{\mathrm{r}}\right)$ and its frequency dependence, as well as the loss tangent. The capacitance versus voltage characteristic was examined and the data were discussed introducing interfacial layers ("dead layers") to describe some of the observations. Special care was taken to describe effects that arise during operation of a DRAM capacitor. These include the dielectric relaxation and the self discharge characteristic of the capacitors. Additionally, leakage current measurements were performed and the results were discussed in terms of a simple Schottky model.

\subsubsection{Properties of the electrical permittivity}

The permittivity values of BST are of major importance, since its high-k properties are the only reason for its nomination as a candidate for the DRAM dielectric in the future chip generations. We discuss first the permittivity issue for films with a thickness around $30 \mathrm{~nm}$, which is close for application, and data from different deposition temperatures and stoichiometries are presented. Further, the problems arising from the permittivity scaling with thickness will be displayed.

\begin{tabular}{|c|c|c|c|c|c|c|c|}
\hline \multirow[t]{2}{*}{ Temp. } & \multicolumn{7}{|c|}{ Stoichiometry $(\mathrm{Gr}-\mathrm{II} / \mathrm{Ti})_{\mathrm{F}}$} \\
\hline & $<0.90$ & $0.91-0.94$ & $0.95-0.98$ & $0.99-1.01$ & $1.02-1.05$ & $1.06-1.09$ & $>1.10$ \\
\hline $670^{\circ} \mathrm{C}$ & $\begin{array}{c}\varepsilon \\
\tan \delta\end{array}$ & & & $\begin{array}{c}190 \\
0,0028\end{array}$ & & & \\
\hline $655^{\circ} \mathrm{C}$ & $\begin{array}{c}120 \\
0,0022 \\
\end{array}$ & $\begin{array}{c}147 \\
0,0020 \\
\end{array}$ & $\begin{array}{c}193 \\
0,0025 \\
\end{array}$ & $\begin{array}{c}171 \\
0,0023 \\
\end{array}$ & $\begin{array}{c}223 \\
0,0023 \\
\end{array}$ & & $\begin{array}{c}228 \\
0,0021\end{array}$ \\
\hline $625^{\circ} \mathrm{C}$ & & $\begin{array}{c}179 \\
0,0022 \\
\end{array}$ & $\begin{array}{c}208 \\
0,0027 \\
\end{array}$ & $\begin{array}{c}148 \\
0,0042 \\
\end{array}$ & $\begin{array}{c}185 \\
0,0029 \\
\end{array}$ & & \\
\hline $595^{\circ} \mathrm{C}$ & & $\begin{array}{c}151 \\
0,0033 \\
\end{array}$ & $\begin{array}{c}188 \\
0,0032 \\
\end{array}$ & $\begin{array}{c}181 \\
0,0033 \\
\end{array}$ & $\begin{array}{c}176 \\
0,0031\end{array}$ & $\begin{array}{c}195 \\
0,0038 \\
\end{array}$ & $\begin{array}{c}192 \\
0,023 \\
\end{array}$ \\
\hline $580^{\circ} \mathrm{C}$ & & & $\begin{array}{c}144 \\
0,0024 \\
\end{array}$ & $\begin{array}{c}160 \\
0,0025 \\
\end{array}$ & $\begin{array}{c}170 \\
0,0034 \\
\end{array}$ & & \\
\hline $565^{\circ} \mathrm{C}$ & $\begin{array}{c}106 \\
0,0028 \\
\end{array}$ & $\begin{array}{c}110 \\
0,0024\end{array}$ & $\begin{array}{c}111 \\
0,0049 \\
\end{array}$ & $\begin{array}{c}107 \\
0,0045 \\
\end{array}$ & $\begin{array}{c}91 \\
0,0039 \\
\end{array}$ & $\begin{array}{c}115 \\
0,005 \\
\end{array}$ & $\begin{array}{c}100 \\
0,0046 \\
\end{array}$ \\
\hline $520^{\circ} \mathrm{C}$ & & $\begin{array}{c}\text { (83) } \\
\text { Thicker film }\end{array}$ & & & & & $\begin{array}{c}38 \\
0.0024\end{array}$ \\
\hline
\end{tabular}

Table 6.5: Selected samples in the temperature-stoichiometry space; they are characterized by the values of the relative permittivity $\left(\varepsilon_{r}\right)$ and loss tangent (tan $\delta$ ). 


\subsubsection{Permittivity values}

Table 6.5 summarizes the typical permittivity and $\tan \delta$ values for samples around $30 \mathrm{~nm}$ and growth temperatures between $565^{\circ} \mathrm{C}$ and $655^{\circ} \mathrm{C}$ in a regime $\pm 10 \%$ from the stoichiometric composition $(G r-I I / T i=1)$ and Fig. 6.49 visualizes the dependence of the dielectric permittivity, $\varepsilon_{\mathrm{r}}$, of the films on the deposition temperature and the film stoichiometry. We observe a strong influence of the deposition temperature on the dielectric constant (or microstructure as shown in the previous sections), e.g. the $\varepsilon_{\mathrm{r}}$ value decreases for lower deposition temperatures. In contrast to earlier investigations [61] we also find a weak increase of the permittivity with higher Gr-II content and the maximum could not be determined in the targeted stoichiometry regime, but there is a faster decrease for highly Ti rich films (Gr$\mathrm{II} / \mathrm{Ti}<0.93)$. At the low temperature regime $\left(565^{\circ} \mathrm{C}\right)$ the permittivity is lower and no longer seriously affected by the film composition. In particular, we obtain a typical value of $\varepsilon \approx 208$ for Ti-rich films $\left(\mathrm{GrII} / \mathrm{Ti} \approx 0.97\right.$ ) grown at $625^{\circ} \mathrm{C}$, which corresponds to a specific capacitance of $\mathrm{C} / \mathrm{A}=59.1 \mathrm{fF} / \mu \mathrm{m}^{2}$. For a film grown at $565^{\circ} \mathrm{C}$ this value is reduced to $\varepsilon_{\mathrm{r}} \approx 110$ or 30.2 $\mathrm{fF} / \mu^{2}$. There are only a few values available for the diel. constant for films deposited at $520^{\circ} \mathrm{C}$. For example the permittivity for a $59 \mathrm{~nm}$ sample with Ti excess (0.94), additionally used for investigation of the conformal growth, is 83 or $12.5 \mathrm{fF} / \mu^{2}$. For the $30 \mathrm{~nm}$ Gr-II rich film we find $\varepsilon_{\mathrm{r}}=38$ and $10.8 \mathrm{fF} / \mu \mathrm{m}^{2}$, respectively.

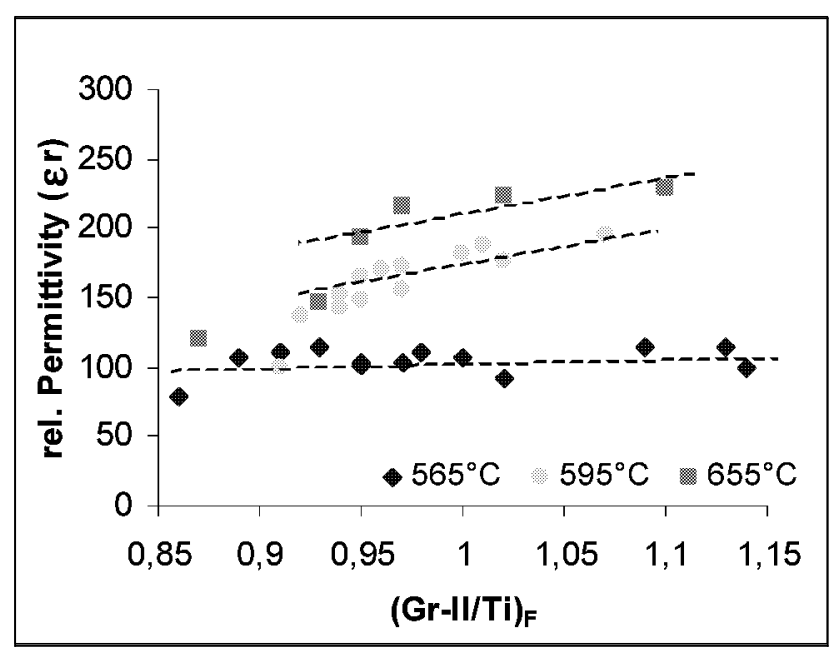

Fig. 6.49: Permittivity $\mathcal{E}_{r}$ as a function of stoichiometry and deposition temperature for $\sim 30 \mathrm{~nm}$ films.

Similar to earlier results we observe an almost constant dispersion of the permittivity over the whole frequency range from $1 \mathrm{~Hz}$ to $1 \mathrm{MHz}$ : e.g. a change of $0.45 \%$ per decade for a Ti-rich film $(\mathrm{GrII} / \mathrm{Ti} \approx 0.97)$ grown at $580^{\circ} \mathrm{C}[34,61]$. More details on the frequency dispersion of the dielectric constant are given in Section 6.2.1.4. Parallel to the permittivity values, we always recorded the loss tangent of the films. Generally, we find excellent values below 0.005 for most of the films and a slight stoichiometry dependence for the high $\mathrm{T}_{\text {growth }}$ region. Additionally, we observe a thickness dependence of the loss tangent. A detailed listing of these results can be found in Section 6.2.2.1.

Fig. 6.50 shows the thickness dependence of the permittivity for samples grown at various deposition temperatures. All samples are Ti-rich and the scattering of the data points can be caused by the somewhat different stoichiometry of the samples as well as due to differences in the topology, e.g. variation of the surface roughness. Nevertheless, the linear fits are 
sufficient to describe the changing in the effective permittivity with thickness with a quite high correlation factor $\left(R^{2}>0.96\right)$ and the extrapolation to zero thickness reveals a non-zero permittivity value. This will be discussed in Section 6.2.1.3 in terms of "dead" or interface layers. An clear increase of the relative permittivity is also observed with higher deposition temperature. This is more obvious for the thicker samples, as the differences in the thinner samples are small. On the other hand, the fit for the dielectric constant of the $565^{\circ} \mathrm{C}$ grown samples is clearly below the values of the high- $T_{\text {growth }}$ samples and defines the boundary between polycrystalline and strongly textured films.
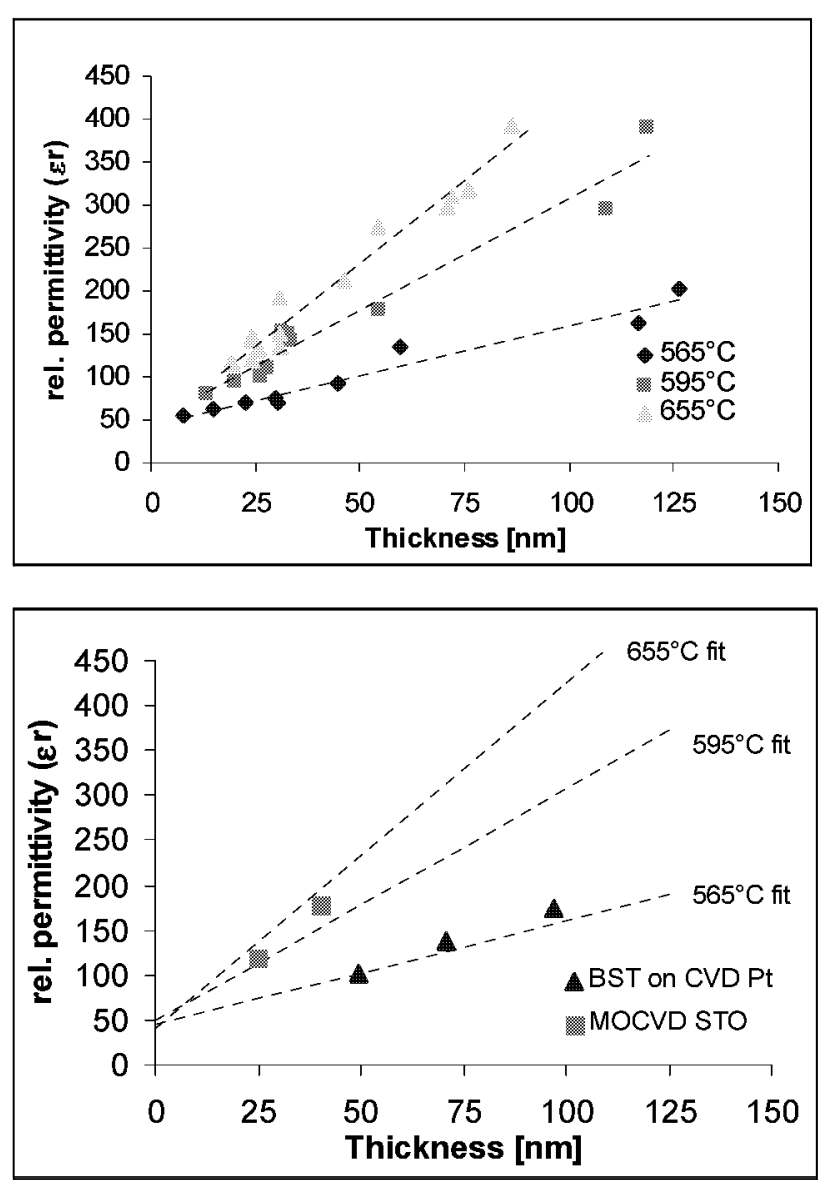

Fig. 6.50: Thickness dependence of the dielectric constant for crystallographic perfect samples deposited at high temperature. Extrapolation for zero thickness gives a non zero value for the permittivity regardless of the deposition temperature of the films, which can be explained with the existence of thin interface layers of low permittivity.

Fig. 6.51: Permittivity values of a) $B S T$ on CVD Pt substrates and b) MOCVD $\mathrm{SrTiO}_{3}$. The linear fits in the graph represent the decrease of the dielectric constant with thickness for samples grown at three different temperatures derived from Fig. 6.50.

In Fig. 6.51 we present the permittivity values for BST samples grown on MOVCD Pt substrates provided by AIXTRON within the HECTOR300 project, as well as strontium titanate $\left(\mathrm{SrTiO}_{3}, \mathrm{STO}\right)$ samples on standard (111) textured Pt wafers. As a comparison, we included the fits for the permittivity of standard BST samples taken from the Fig. 6.50. Starting with the STO films grown at $655^{\circ} \mathrm{C}$, we obtain a similar behavior with the BST samples for high deposition temperatures, at least for this narrow thickness band from 25 to $40 \mathrm{~nm}$. Thus, the dielectric constant for a $40 \mathrm{~nm}$ STO film has a value of 180 . On the other hand, the BST samples grown on CVD Pt at $595^{\circ} \mathrm{C}$ display a much lower permittivity that the BST samples grown at similar conditions on (111) textured $\mathrm{Pt}$ and their values match those from the $565^{\circ} \mathrm{C}$ grown samples. The tan $\delta$ value of the BST films grown on MOCVD Pt is in the range of $6-7 \%$. 


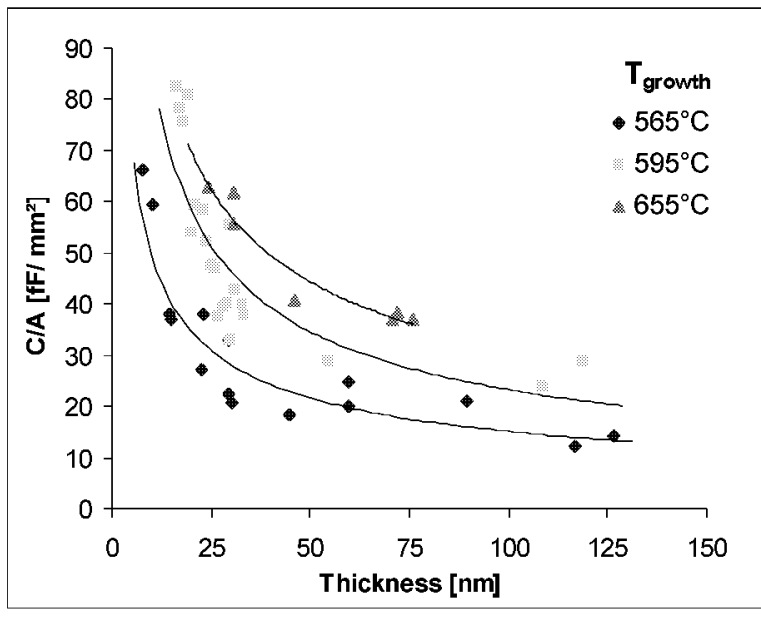

Fig. 6.52: Specific capacitance vs. thickness for samples grown at different temperatures.

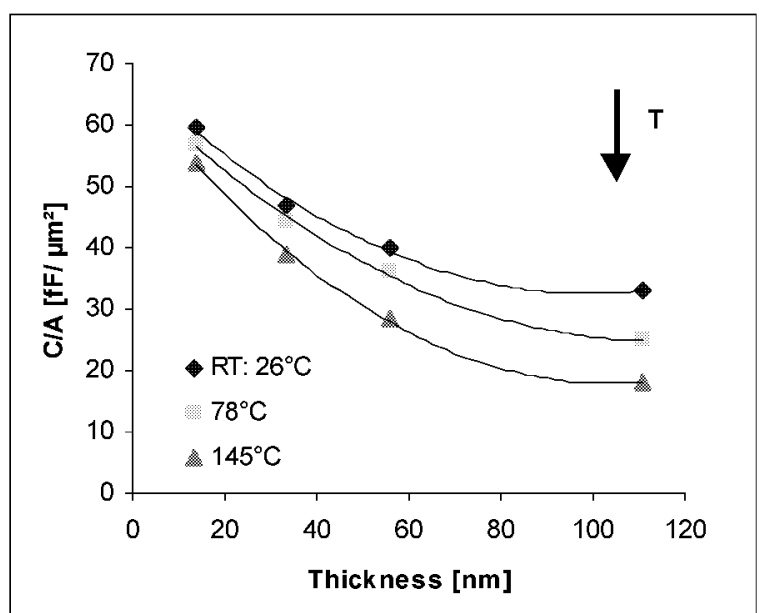

Fig. 6.53: Specific capacitance vs. thickness at different temperatures for stoichiometric samples $\left(T_{\text {growth }}=595^{\circ} \mathrm{C}\right)$.

Fig. 6.52 depicts the dependence of the specific capacitance on the growth temperature and Fig. 6.53 displays the influence of the environment temperature on the specific capacitance. In both cases we observe typical nearly asymptotic fits for zero thickness. The values that enter in these plots are taken at zero bias and consequently do not always represent the maximum capacitance for a given geometry. Same as in the $\mathrm{C}-\mathrm{V}$ plot for different deposition temperatures (see next section), we clearly observe higher capacitance density values with higher deposition temperature. According to the plot in Fig. 6.52 , the $\mathrm{C} / \mathrm{A}$ values at $655^{\circ} \mathrm{C}$ are nearly twice as high as for the $565^{\circ} \mathrm{C}$ grown samples. Concerning the temperature dependence of the capacitance for films of different thickness, Fig. 6.53 presents the capacitance area density versus thickness graph for stoichiometric films grown at $595^{\circ} \mathrm{C}$. While the decrease of the permittivity with temperature at zero bias is quite significant for the samples with higher thickness, this does not seem critical for very thin samples. This behavior is very helpful in an application environment, e.g. a thin dielectricum would allow a more stable operation of the DRAM capacitor.

\subsubsection{C-V characteristic and tunability}

The voltage dependent capacitance properties were investigated as a function of temperature and film thickness. The capacitance-voltage analysis (C-V) was performed using multiple cycle sweeps, from zero bias to maximum positive voltage, back to the maximum negative voltage and finally back to zero, schematically: $0 \rightarrow+\rightarrow 0 \rightarrow-\rightarrow 0$, in order to check for possible hysteresis loops. The examined samples with a thickness between $15-126 \mathrm{~nm}$ did not show any hysteretic behavior in the temperature region from $25-145^{\circ} \mathrm{C}$, that was considered as close to the application in integrated circuits. The measurements were performed at the frequency of $1 \mathrm{kHz}$ with an oscillation level of $50 \mathrm{mV}$. This was chosen appropriately not to suppress the permittivity near zero bias, and to obtain a comfortable signal-to-noise ratio. All films display a nonlinear nonhysteretic dielectric response as described by the widely accepted LGD theory [36] and verified by other groups for sputtered and MOCVD BST films, regardless of their deposition temperature [35]. The observed thickness dependency of the C$\mathrm{V}$ plots as well as the already mentioned thickness dependent permittivity of our films, that is attributed to intrinsic interface layers of low permittivity, lead to the assumption that the films are fully depleted [100]. 
At first we discuss the influence of the deposition temperature on the capacitance area density and consequently on the dielectric constant of our films. For both low and high growth temperature regimes, we measured the small signal $\mathrm{C}-\mathrm{V}$ characteristic for samples with different thickness. From the aspect of charge storage devices, it is important to integrate the $\mathrm{C}-\mathrm{V}$ curves, thus obtaining the polarization charge. The temperature dependency of the $\mathrm{C}-\mathrm{V}$ curves was further studied in a range interesting for application $\left(25-145^{\circ} \mathrm{C}\right)$. The obtained data are then plotted in terms of the dead-layer model, which implies the existence of thin interface layers of reduced permittivity.

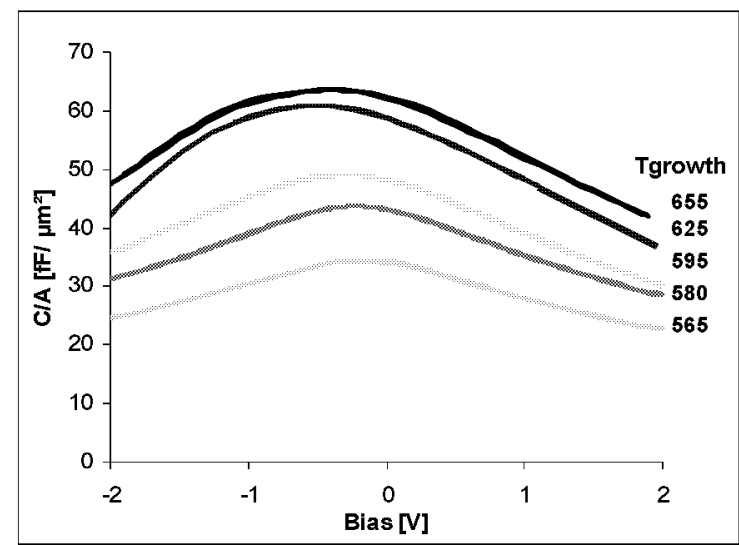

Fig. 6.54: $C / A(V)$ curves for Ti-rich samples (GrII/Ti $=0.95-0.97)$ grown between 565 and $655^{\circ} \mathrm{C}$.

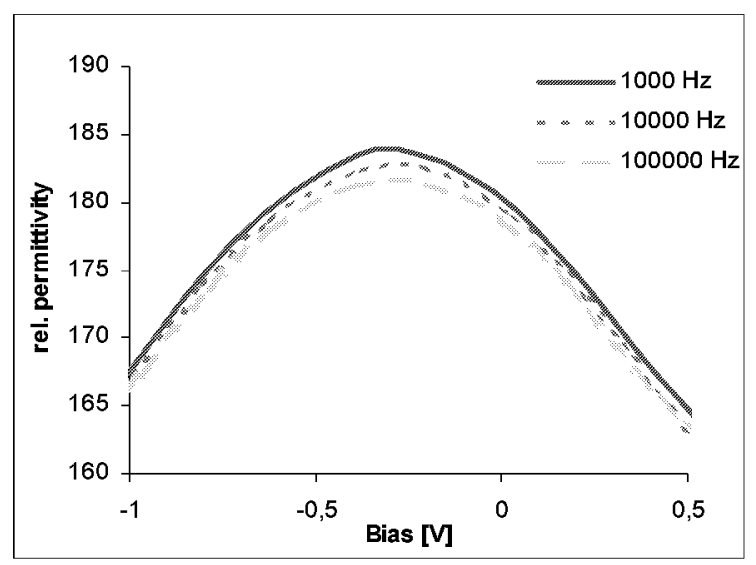

Fig. 6.55: Dispersion of the permittivity as depicted in the $\varepsilon(V)$ characteristic.

Fig. 6.54 shows the bias voltage dependence of the specific capacitance for Ti rich samples $(\mathrm{GrII} / \mathrm{Ti} \approx 0.97)$ deposited between 565 and $655^{\circ} \mathrm{C}$. The obtained curves are asymmetric with an additional offset of the curve maximum of approx. $0.2 \mathrm{~V}$ to negative values. This effect seems related to our present electrode deposition and treatment and is under investigation. It seems that the shift becomes larger with increasing temperature and no explanation is known at the present. The magnitude of the capacitance is also scaling with the deposition temperature, an effect that is attributed to the different crystallinity grades of the films.

The influence of the frequency can also be seen in the $\mathrm{C}-\mathrm{V}$ characteristic shown in Fig. 6.55 for three different frequencies. A magnified sector is used to depict the dispersion of the capacitance values, since the effect is too small to visualize in the standard $\mathrm{C}-\mathrm{V}$ plots.

Fig. 6.56a-d displays the $\mathrm{C}-\mathrm{V}$ characteristic of Ti-rich films grown at different temperatures $\left(565^{\circ} \mathrm{C}-595^{\circ} \mathrm{C}\right)$. Every plot includes samples of different thickness in the range between $20-$ $130 \mathrm{~nm}$. Gr-II rich compositions were examined in the case of the $595^{\circ} \mathrm{C}$ samples. The figure shows the appropriate plots as-measured, without any zero point correction, in order to achieve fully symmetrical plots. This could be achieved by subtracting the offset from the field values, but does not represent the true applied field across the film. 


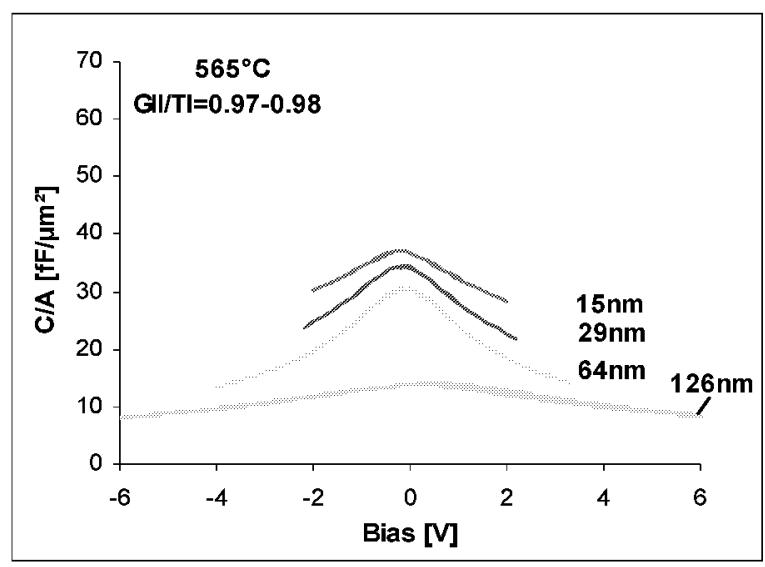

Ti-rich samples; $T_{\text {growth }}=565^{\circ} \mathrm{C}$

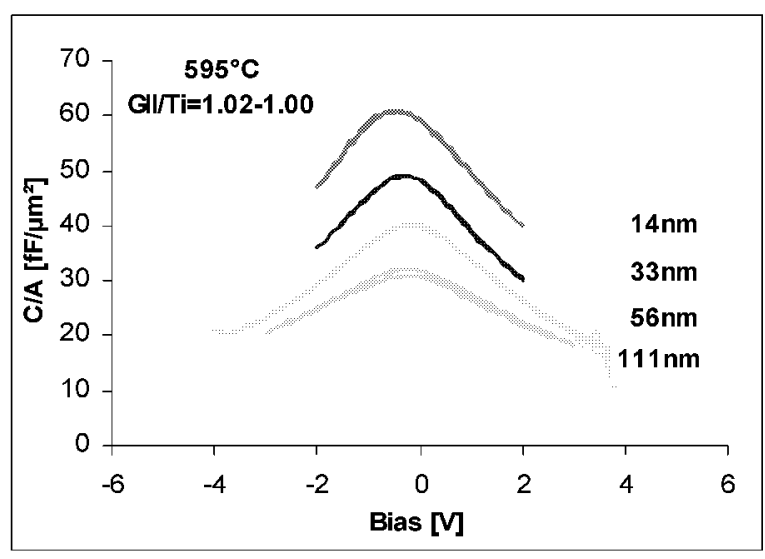

Gr-II rich samples; $T_{\text {growth }}=595^{\circ} \mathrm{C}$

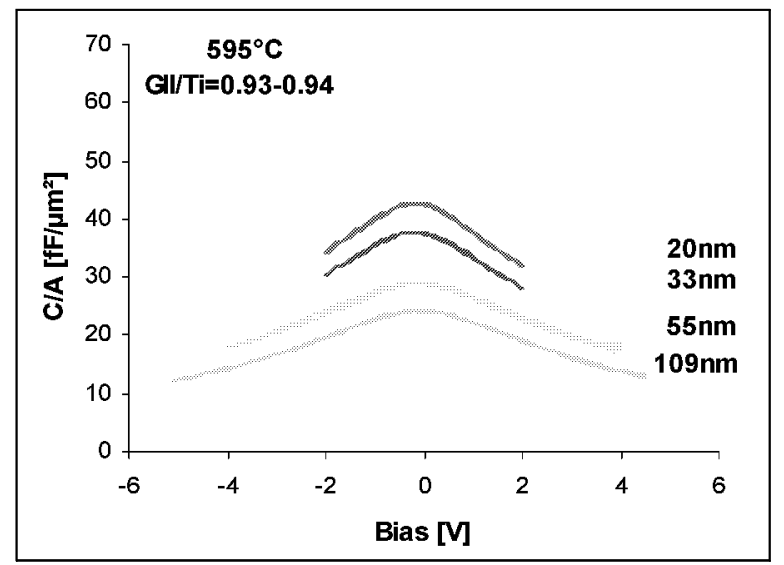

Ti-rich samples; $T_{\text {growth }}=595^{\circ} \mathrm{C}$

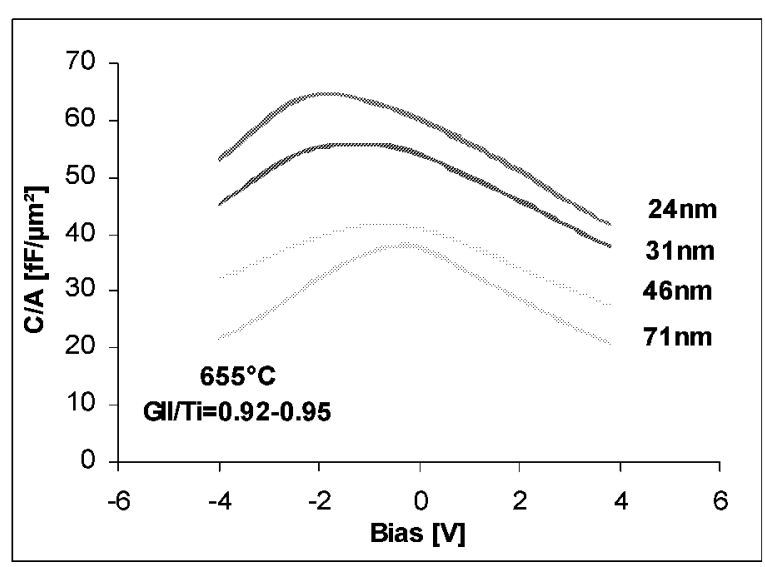

Ti-rich samples; $T_{\text {growth }}=655^{\circ} \mathrm{C}$

Fig. 6.56: Capacitance-Voltage plots for thickness series of different growth temperature and stoichiometry; the maximum of the curves is mostly shifted to negative voltage values.

The nonlinearity in the capacitance is clearly visible and can find application in tunable microwave devices. Resulting from this nonlinearity the available change for DRAM applications is less than the value calculated at zero bias. The complete voltage sweep is depicted in the graphs to show the lack of hysteretic behavior.

The available charge in a BST capacitor is given by integrating the small signal C-E curves. This is achieved by fitting a polynomial function of the $n$-th order (usually $n=4$ or 5 ) to the $C$ $\mathrm{V}$ curve. The charge $Q$ can then be calculated according to:

$$
Q=\int_{V 1}^{V 2} C(V) d V
$$

This is shown for the $20 \mathrm{~nm}$ Ti-rich sample $\left(\mathrm{T}_{\text {growth }}=595^{\circ} \mathrm{C}\right)$ in Fig. 6.57, which shows the charge in the capacitor vs. the applied voltage. The nonlinearity in the $\mathrm{Q}-\mathrm{V}$ characteristic comes from the nonlinear $\mathrm{C}-\mathrm{V}$ plot. In the same figure the dashed line through zero is calculated for the same virtual linear capacitor. Assuming a $2 \mathrm{~V}$ voltage drop across the BST capacitor in the DRAM operation, the capacitor averages approx $10 \%$ less charge compared to the linear case. 


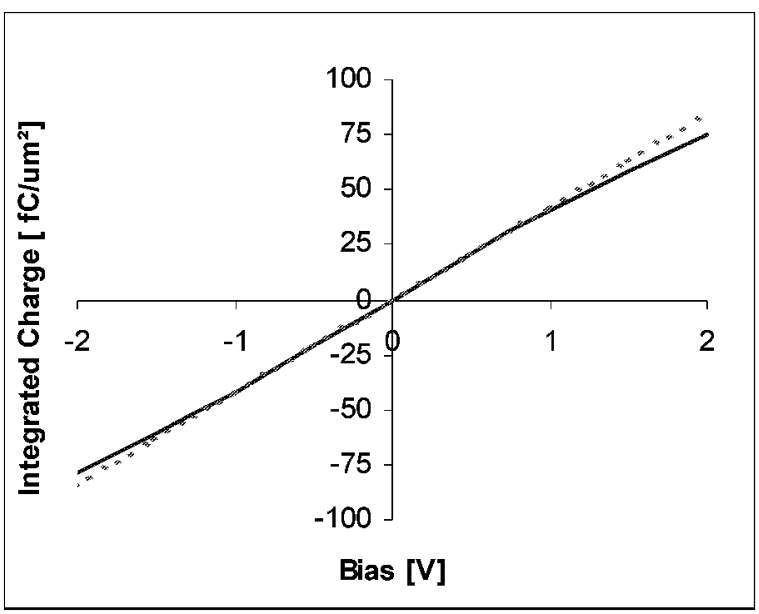

Fig. 6.57: Charge density vs. applied voltage for the $20 \mathrm{~nm}$ sample.

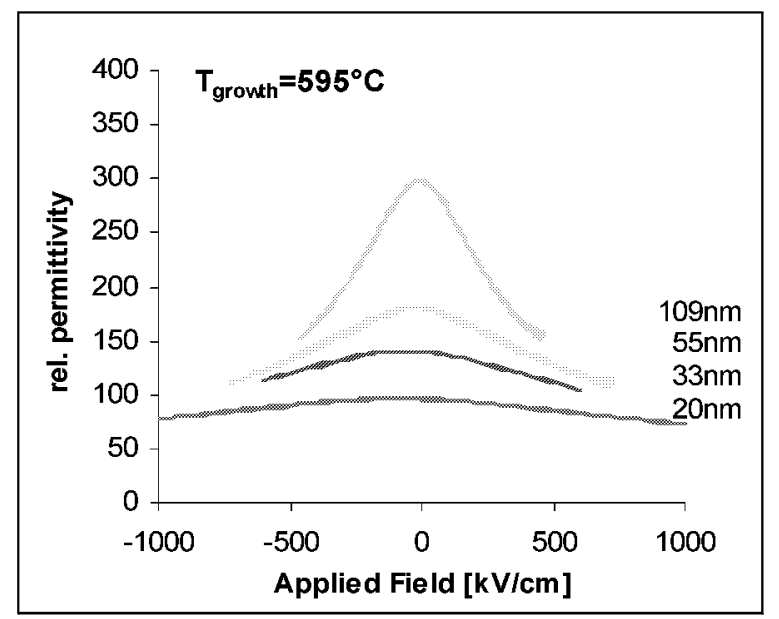

Fig. 6.58: Influence of the applied field on the dielectric constant as a function of thickness.

The already mentioned shift of the $\mathrm{C}-\mathrm{V}$ curves to a negative voltage can be up to $0.5 \mathrm{~V}$ large and is consistent with a non-uniform distribution of charge in the film during capacitor manufacturing and especially through asymmetrical top and bottom electrodes. This also causes the asymmetry in Fig. 6.57, where a greater charge loss is found for a positive voltage than for a negative one, e.g. $12 \%$ loss at $2 \mathrm{~V}$ and $8 \%$ at $-2 \mathrm{~V}$.

The influence of the thickness on the permittivity at zero bias has already been shown, e.g. $\varepsilon_{\mathrm{r}}$ decreases with lower thickness. This is depicted here for Ti-rich films at $595^{\circ} \mathrm{C}$ as a function of the applied field (see Fig. 6.58). The difference is prominent for a small signal operation, however, it becomes smaller with increasing electrical field, until we obtain a near thickness independent dielectric constant for high applied fields (extrapolated value: $>1000 \mathrm{kV} / \mathrm{cm}$ ). Additionally, we observe a deviation from the extreme nonlinear behavior while moving to lower thicknesses and the measurement on the $20 \mathrm{~nm}$ Ti-rich sample is almost independent from the electrical field.

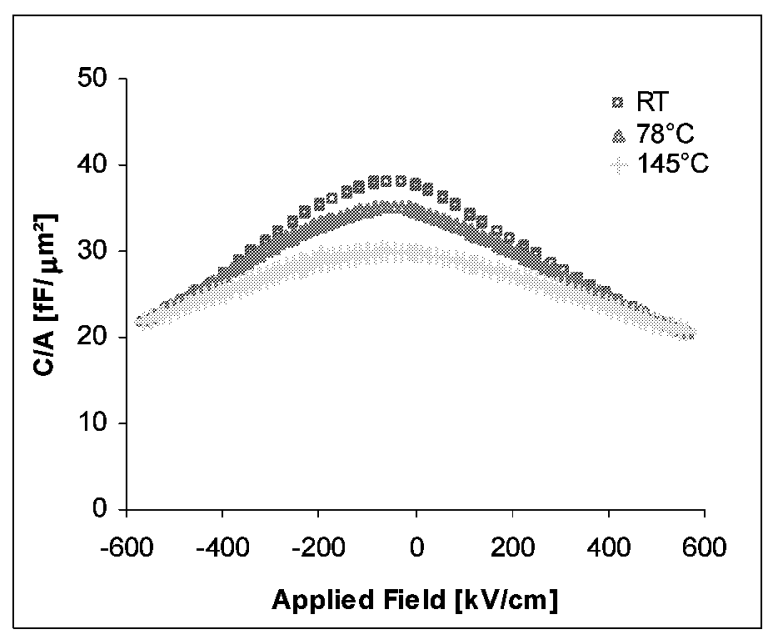

Fig. 6.59: C-E characteristic of a $71 \mathrm{~nm}$ sample $\left(655^{\circ} \mathrm{C}\right)$ for various temperatures. There is a clear flattening of the curve with increasing temperature and the capacitance area density remains stable for higher fields.

The operation temperature has a significant impact on the C-V characteristic as shown in Fig. 6.59 for a Ti-rich $71 \mathrm{~nm}$ sample grown at $655^{\circ} \mathrm{C}$. The zero-volt capacitance value and 
consequently the permittivity that is measured on that position drops $7 \%$ at $78^{\circ} \mathrm{C}$ and $20 \%$ at $145^{\circ} \mathrm{C}$ compared to room temperature (about $26^{\circ} \mathrm{C}$ ). The capacitance area density and consequently the permittivity clearly decreases with temperature near zero bias. This effect becomes weaker with higher fields until the permittivity is independent from temperature, an issue that is very important for stable device operation.

Since we generally obtain a non linear dielectric behavior, it is possible to use these films in tunable devices. The grade of the tunability is an important issue that is defined by Eq. 6.6. The equation determines the tunability of the permittivity of a material between zero and a certain bias voltage $x$.

$$
\begin{array}{ll}
\text { Tunability }=\frac{\varepsilon_{r}(0 V)-\varepsilon_{r}(x V)}{\varepsilon_{r}(0 V)} \cdot 100 \% \quad \text { Eq. } 6.6
\end{array}
$$

It is problematic to compare the tunability of samples with different thicknesses, that result into different applied fields for a certain bias. However, it is possible to compare the values for films with the same thickness. The calculation of the tunability for the samples with a thickness around $30 \mathrm{~nm}$ resulted into values $\sim 35 \%$ with the Ti-rich sample displaying a significant lower tunability of $25 \%$. Generally, we expect an enhancement of the tunability with thickness. This can be understood by the thickness dependence of the permittivity in Fig. 6.56. Hence, it is necessary to plot the field dependence of the permittivity, in order to compare samples with different thicknesses (see Fig. 6.58). In this case, the tunability equation has to be modified, to contain the field instead of the voltage values. The tunability for the Ti-rich samples grown at $595^{\circ} \mathrm{C}$ for an applied field of $400 \mathrm{kV} / \mathrm{cm}$ is presented in the next table, which confirms that the higher the thickness, the sharper the decrease of the permittivity.

\begin{tabular}{|c|c|c|c|}
\hline $\begin{array}{l}595^{\circ} \mathrm{C} \text { Ti-rich } \\
\text { thickness [nm] }\end{array}$ & $\begin{array}{c}\text { Tunability [\%] } \\
1 \mathrm{kHz}-400 \mathrm{kV} / \mathrm{cm}\end{array}$ & $\begin{array}{c}655^{\circ} \mathrm{C} 71 \mathrm{~nm} \text { Ti-rich } \\
\text { Temperature }\left[{ }^{\circ} \mathrm{C}\right]\end{array}$ & $\begin{array}{c}\text { Tunability [\%] } \\
1 \mathrm{kHz}-4 \mathrm{~V}\end{array}$ \\
\hline 20 & 8 & 25 & 45 \\
\hline 33 & 15 & 78 & 41 \\
\hline 55 & 23 & 145 & 31 \\
\hline 109 & 46 & & \\
\hline
\end{tabular}

Table 6.6: Tunability values as a function of a) film thickness b) operation temperature

An increase of the operation temperature leads to the decrease of tunability, as can be seen in Fig. 6.59. Concerning the tunability between $0-4 \mathrm{~V}$ of a $71 \mathrm{~nm}$ Ti-rich sample grown at $655^{\circ} \mathrm{C}$, we obtain a strong decrease from $45 \%$, measured at room temperature, to $31 \%$, measured at $145^{\circ} \mathrm{C}$, which is approx. $30 \%$ of the initial value.

\subsubsection{Dead Layer Model}

In the literature the most common model for the origin of the thickness dependence of the permittivity are interfacial layers, or "dead layers", that act as capacitors in series influencing the effective permittivity $\mathrm{C}_{\text {eff }}$ of the BST capacitor [101]. According to the "dead layer" model the capacitor consists of a bulk region $C_{\mathrm{B}(\mathrm{ulk})}$, and the two interfaces to the electrodes $C_{i}$, that can be described by two capacitors of a lower permittivity. Fig. 6.60 presents the resulting configuration and Eq. 6.7 the resulting reciprocal capacitance of a BST film. Hence, 
in a plot of $1 / \mathrm{C}$ versus thickness the bulk permittivity is given by the slope, while the intercept reveals the interface capacitance.

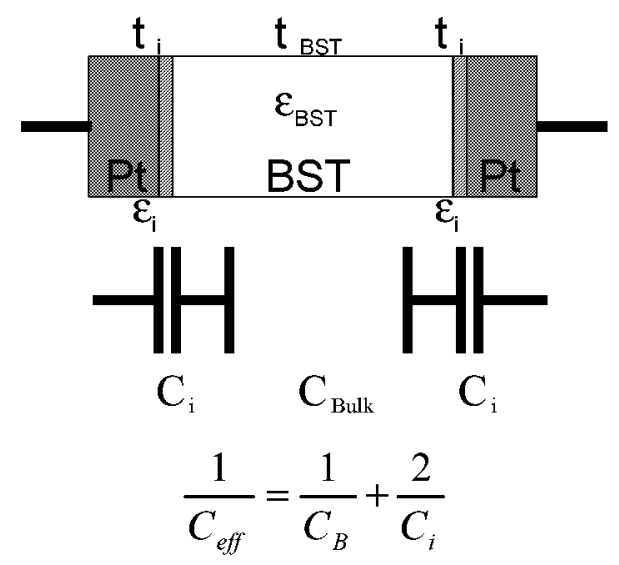

Fig. 6.60: Equivalent electrical network of a BST thin film based on the "dead layer" model.

Substituting for the capacitance $\left(C=\varepsilon_{0} \varepsilon A / t\right)$, where $A$ is area, $t$ the thickness and $\varepsilon_{0}$ permittivity of the free space, yields the final equation:

$$
\frac{A}{C_{e f f}}=\frac{2 \cdot A}{C_{i}}+\frac{A}{C_{B}}=\frac{2 \cdot t_{i}}{\varepsilon_{0} \cdot \varepsilon_{i}}+\frac{\left(t-2 \cdot t_{i}\right)}{\varepsilon_{0} \cdot \varepsilon_{B}}
$$

where $C_{\mathrm{B}}$ the bulk and $C_{\mathrm{i}}$ the interface capacitance respectively. $\varepsilon_{\mathrm{i}}$ and $t_{\mathrm{i}}$ represent the permittivity of the interface and its thickness, and $\varepsilon_{B}$ is the permittivity of the bulk. No information is known for the thickness and the permittivity of the dead layers, only for their contribution in the serial interface capacitance. Hence, modeling of the interface is only possible by setting different values for one parameter and discussing the influence on the second for a certain interface capacitance. The linear fit of the values in the $\mathrm{A} / \mathrm{C}$ vs. thickness plots implies that the interface thickness is small compared to the total film thickness, e.g. $t_{\text {total }}-2 \cdot t_{\text {int }} \sim t_{\text {total }}$. In other words, the bulk thickness is similar to the total film thickness. Additionally, the low permittivity interface layer leads to an increase of the electrical field close to the electrode $E_{\mathrm{i}}$, compared to the field in the bulk region $E_{\mathrm{BST}}$, according to:

$$
E_{\mathrm{i}}=E_{\mathrm{BST}} \cdot \varepsilon_{\mathrm{BST}} / \varepsilon_{\mathrm{i}} \text { Eq. } 6.9
$$

The higher field near the electrode causes an enhanced barrier lowering $\Delta \phi$, as well as higher relative permittivity $\varepsilon_{\mathrm{r}}$, in the standard Schottky model. The latter will be described in detail along with the discussion of the leakage current measurements on BST thin films (Section 6.2.2.2).

Despite the rather large scattering of the data points, there are indications that the linear fit is insufficient to describe the behavior of samples with very small thicknesses $(<30 \mathrm{~nm})$. This is similar to the observations from other groups meaning that the model need to be adjusted [102] and more research needs to be done towards this direction. Nevertheless, the phenomenological model yields a good overall description of the data. The origin of the interface layers is currently under debate. Possible reasons include extrinsic effects like surface contamination through impurities due to the lack of cluster tools for complete processing, reaction layers during nucleation of the BST or interdiffusion of foreign elements, as well as intrinsic effects, based on the influence of the boundary on soft phonon characteristics [112] or the electric field penetration on the metal side [113]. The microstructural investigation of our films using high resolution HRTEM and SEM tools did not display any type of interfacial layers and the XRD patterns could not reveal any second phases and intrinsic effects may be favoured. 
The model was applied to the permittivity values of Ti-rich films with different thicknesses for three growth temperatures $\left(565^{\circ} \mathrm{C}, 595^{\circ} \mathrm{C}\right.$ and $\left.655^{\circ} \mathrm{C}\right)$. Parallel investigations on three series of Ti-rich, Gr-II-rich and stoichiometric samples grown at $595^{\circ} \mathrm{C}$ were performed. Fig. 6.61a shows the plots for the different thickness series and table 6.7 summarizes the results for the bulk and interface permittivity. The bulk permittivity shows a strong increase from $\sim 250$ at $565^{\circ} \mathrm{C}$ to approx. 860 at $595^{\circ} \mathrm{C}$ and seems constant within the uncertainties at higher growth temperatures. This is attributed to the major differences in the microstructure observed between low and high temperature deposition. The interface capacitance $(\mathrm{Ci} / 2 A)$ shows a slight but systematic increase with deposition temperature for the Ti-rich samples. In spite of the scattering of the data there is an indication for a higher interface capacitance of the Gr-IIrich samples deposited at $595^{\circ} \mathrm{C}$. Fig. $6.61 \mathrm{~b}$ shows the reciprocal capacitance vs. thickness for the $595^{\circ} \mathrm{C}$ samples of different stoichiometry. The plot provides an indication that a trade off must be found between the decreasing permittivity values with higher GII-ratio and the parallel rising interface capacitance. Thus, the stoichiometric samples display acceptable values both in terms of bulk permittivity and interface capacitance.

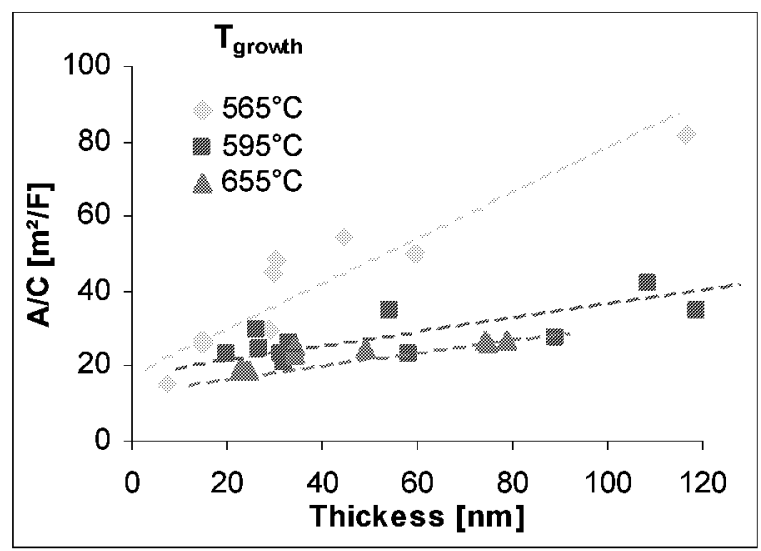

a) Thickness dependence of the reciprocal permittivity and extrapolation from the linear fit.

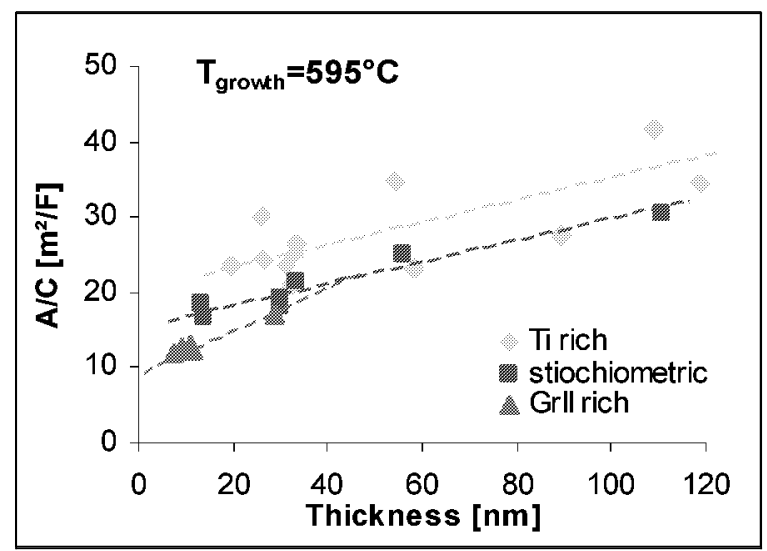

b) Reciprocal capacitance vs. thickness for samples of different stoichiometry grown at $595^{\circ} \mathrm{C}$.

Fig. 6.61: Application of the dead layer model to the permittivity data of our MOCVD grown films.

\begin{tabular}{|c|c|c|c|}
\hline $\begin{array}{c}\text { Growth Temp } \\
\left({ }^{\circ} \mathrm{C}\right)\end{array}$ & GII/Ti ratio & $\begin{array}{l}\text { Interface cap. } \\
\left(\mathrm{fF} / \boldsymbol{\mu m}^{2}\right)\end{array}$ & $\begin{array}{c}\text { Bulk } \\
\text { Permittivity }\end{array}$ \\
\hline 565 & $0.90-0.98$ & 86 & 249 \\
\hline 595 & $0.93-0.97$ & 95 & 862 \\
\hline 595 & $0.98-1.02$ & 121 & 865 \\
\hline 595 & $1.03-1.11$ & 193 & 489 \\
\hline 655 & $0.91-0.95$ & 122 & 837 \\
\hline 655 & $1.03-1.10$ & 212 & 591 \\
\hline
\end{tabular}

Table 6.7: Summary of the bulk permittivity and interface capacitance $(\mathrm{C} \mathrm{i} / 2 \mathrm{~A})$ values extracted from Fig. 6.61 using the dead layer model.

Equivalent differences between Ti- and Gr-II rich films are also obtained for the samples grown at $655^{\circ} \mathrm{C}$, thus leading to the conclusion that the observed behavior is real. Hence, we may conclude that a Gr-II rich interface, which would result into higher interface capacitance, 
followed by a Ti-rich bulk that would add a higher bulk dielectric constant, yield an enhanced effective permittivity of the stack. An explanation in terms of differences in the film morphology issued for non-stoichiometric compositions as presented in Section 6.1.3 remains highly speculative. According to microscopy results, we obtain locally larger surface roughness for Gr-II rich films, attributed to the isolated grain growth. This can lead to a larger effective surface area, which enters the calculation for the capacitance area density, and consequently to a larger interface capacitance. On the other hand, the difference in the columnar structure can affect the bulk permittivity.

Compared with results from other international groups the previously discussed bulk permittivity values are excellent and only the interface capacitance of our films seems to be significant lower by a factor of two than the reported values. Basceri reported in 1997 an interface capacitance of $\sim 240 \mathrm{fF} / \mu \mathrm{m}^{2}$ and a permittivity value for the bulk $\varepsilon_{\text {bulk }}<350$ for Tirich films grown at $640^{\circ} \mathrm{C}$ [35]. In 1999, Kotecki measured an interface capacitance of 180$230 \mathrm{fF} / \mu^{2}$ and the results for the bulk permittivity were in the order of 400-500 [9]. The comparison to our results indicates that their films were Gr-II rich in contrast to the original statements. This discrepancy demonstrates again the importance of exact film analytics. A comparison with samples produced by the CSD method, where the stoichiometry should be more precise, shows a specific capacitance of $136 \mathrm{fF} / \mu \mathrm{m}^{2}$ for fiber grained stoichiometric BST films, which is similar to the values found for our stoichiometric MOCVD films deposited at $595^{\circ} \mathrm{C}$.

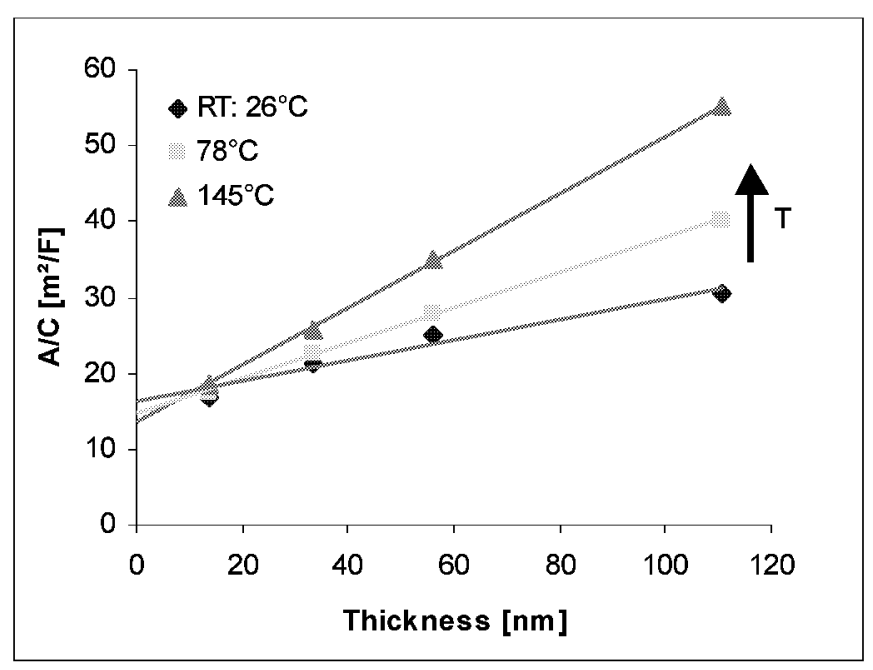

Fig. 6.62: $A / C$ vs. thickness for the stoichiometric $595^{\circ} \mathrm{C}$ samples at different temperatures. According to the dead layer model the interface capacitance is not affected by temperature, in contrast to the bulk permittivity.

Fig. 6.62 presents the data for stoichiometric samples grown at $595^{\circ} \mathrm{C}$ for different measurement temperatures in a plot according to the "dead-layer" model. Within the uncertainties the interface capacitance seems to remain constant at higher temperatures with a mean value of $142 \mathrm{fF} / \mu^{2}$, while the bulk permittivity drops significantly from 840 at room temperature to 300 at $145^{\circ} \mathrm{C}$. The different temperature dependence of the bulk permittivity and the interface capacitance indicates the presence of different effective mechanisms and supports the application of the "dead layer" model.

\subsubsection{Frequency dependence}

The frequency dispersion of the permittivity was studied on samples grown on different substrates, at different temperatures, with different stoichiometries and thickness. All measured films, regardless of the process conditions or their composition, generally displayed 
a stable dispersion of the permittivity with frequency. The data was plotted in typical single logarithmic permittivity vs. frequency graphs.

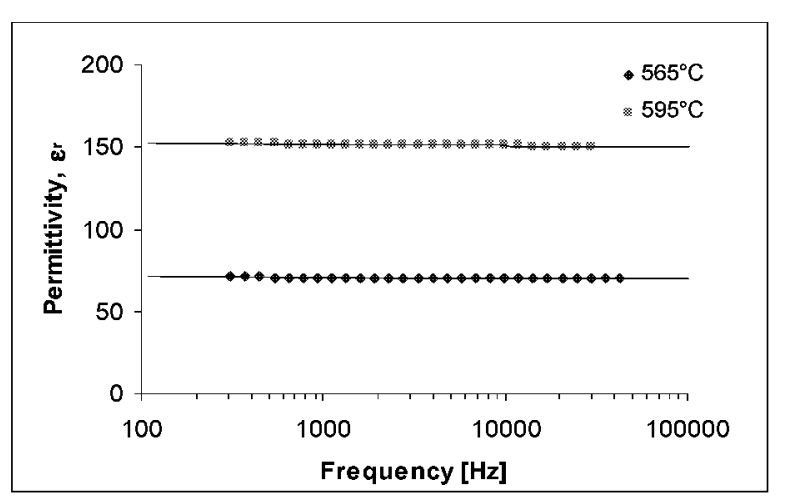

Fig. 6.63: $30 \mathrm{~nm}$ films grown at different temperatures displaying minor permittivity dependence on frequency.

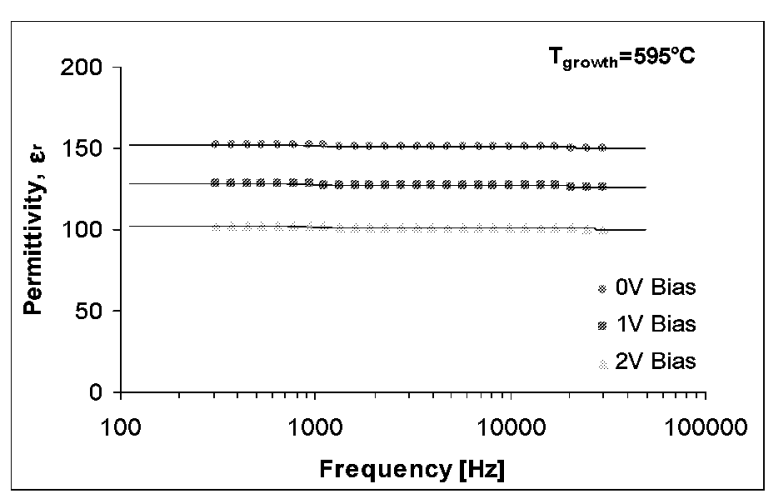

Fig. 6.64: Influence of the bias voltage on the dispersion of the dielectric constant recorded on a $595^{\circ} \mathrm{C}$ grown $30 \mathrm{~nm}$ sample.

The dispersion of the dielectric permittivity was generally in the order of a $0.5 \pm 0.2 \%$ decrease pro decade for the $0 \mathrm{~V}$ bias case. Nevertheless, within the precision of the measurement some dependencies are visible within this narrow region. By comparing two 30nm Ti-rich films grown at $565^{\circ} \mathrm{C}$ and $595^{\circ} \mathrm{C}$ respectively, we obtain a small increase in the dispersion of the dielectric constant for the sample deposited at the higher temperature (Fig. 6.63). For the same samples, the frequency dispersion of the permittivity was recorded while driving the bias voltage from 0 to $2 \mathrm{~V}$ (Fig. 6.64) and a constant increase with increasing voltage load was found. Table 6.8 presents the measurement results of the dispersion experiments for the samples mentioned previously. There is an obvious increasing trend for the dispersion of the dielectric constant towards higher bias voltage values.

\begin{tabular}{|c|c|c|c|}
\cline { 2 - 4 } \multicolumn{1}{c|}{} & \multicolumn{3}{c|}{ Dispersion [\%/decade] } \\
\hline $\mathrm{T}_{\text {growth }}\left[{ }^{\circ} \mathrm{C}\right.$ ] & $0 \mathrm{~V}$ & $1 \mathrm{~V}$ & $2 \mathrm{~V}$ \\
\hline 565 & 0.46 & 0.60 & 0.65 \\
\hline 595 & 0.58 & 0.66 & 0.78 \\
\hline
\end{tabular}

Table 6.8: Permittivity dispersion as a function of growth temperature and bias voltage

Within the precision of our measurements we could not determine any thickness or stoichiometry dependence on the permittivity dispersion. Regarding its temperature dependence in the region between $25^{\circ} \mathrm{C}-145^{\circ} \mathrm{C}$, slight variations are observed, but conflicting trends do not allow a general statement. In the literature one can find a slight temperature dependence in the dispersion of the dielectric constant from $0.38 \%$ to $0.44 \%$ per decade between $25^{\circ}$ and $125^{\circ} \mathrm{C}$ [6]. Based on the acquired data measured at the low frequency region we can extrapolate the value of the dielectric constant for higher frequency operation. By assuming a mean dispersion of $0.5 \%$ pro decade at $1 \mathrm{~V}$ bias the permittivity value at $1 \mathrm{GHz}$ would be just $4 \%$ lower than the measured value at $1 \mathrm{kHz}$, which is usually the value for standard characterization. 

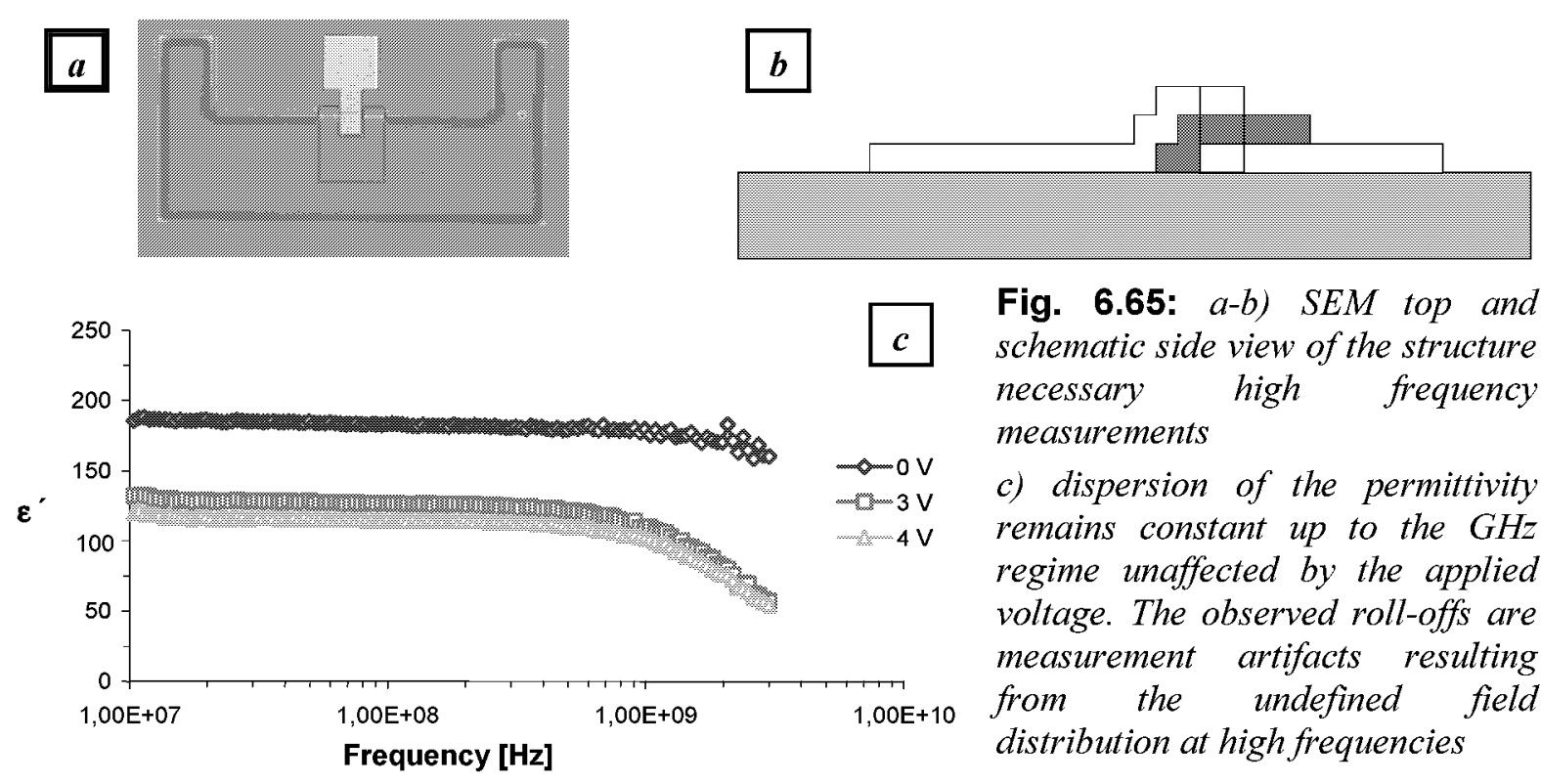

Fig. 6.65: a-b) SEM top and schematic side view of the structure necessary high frequency measurements

c) dispersion of the permittivity remains constant up to the $\mathrm{GHz}$ regime unaffected by the applied voltage. The observed roll-offs are measurement artifacts resulting from the undefined field distribution at high frequencies

These previous measurements were performed on planar capacitors using standard tungsten contact needles and the HP 4284A LCR meter in the region from $10^{2}$ to $10^{5} \mathrm{~Hz}$. In order to perform more precise frequency measurements up to the microwave region $(\leq 3 \mathrm{GHz})$, Giannas used the especially designed MIM structures shown in Fig. 6.65 [33]. The measurements were performed using the network parameter analyzer HP 8753B. The measurements were optimized for a capacitor area of $20 \times 20 \mu \mathrm{m}^{2}$, which is much smaller than the dimensions of our capacitor structures mentioned above, that were measured using the conventional technique.

For a Ti-rich MOCVD BST sample of $73 \mathrm{~nm}$ thickness grown at $595^{\circ} \mathrm{C}$ the dispersion was $\sim 2.5 \%$ /decade, which is higher than the previous values and other published values by approximately a factor five. High frequency measurements depend critically on the structure design and the measurement technique and more work needs to be done in this direction, since the high frequency characteristic is absolutely essential for the development of integrated capacitors. Besides the extrapolations in the high frequency regimes, there are investigation from other groups up to the $20 \mathrm{GHz}$ region. Baniecki obtains a decrease of the permittivity of less than $7 \%$ between $1 \mathrm{mHz}$ and $20 \mathrm{GHz}$, that corresponds to a constant dispersion of $\sim 0.54 \% /$ decade over 13 orders of magnitude in frequency. $\tan \delta$ remains almost unchanged over this region [34]. As a direct consequence, the dispersion effects of the permittivity are not a limiting factor for the use of BST as the capacitors dielectric in DRAM application.

\subsubsection{Loss mechanisms}

\subsubsection{Loss tangent}

The dissipation factor ( $\tan \delta$ or loss tangent) represents the different loss mechanisms in the films and was monitored for different compositions and thicknesses as a function of the growth temperature. Fig. 6.66 presents the dependence of the loss tangent on the stoichiometry for a certain thickness $(\sim 30 \mathrm{~nm})$ and Fig. 6.67 shows the thickness influence on $\tan \delta$. 
For the $30 \mathrm{~nm}$ films we studied the influence of the stoichiometry on the dissipation factor in detail. We do observe a clear increase of the tan $\delta$ values with higher Gr-II content of a factor 2-3. Nonetheless, the resulting losses are still quite low. For the highest deposition temperature of $655^{\circ} \mathrm{C}$ we always obtain $\tan \delta$ values near 0.002 and the losses increase slightly as the growth temperature decreases, but this transition is not that clear for Ti-rich samples. A reasonable trade off between the rise in the permittivity and the tan $\delta$ value with increasing GrII content appears to exist for the stoichiometric composition.

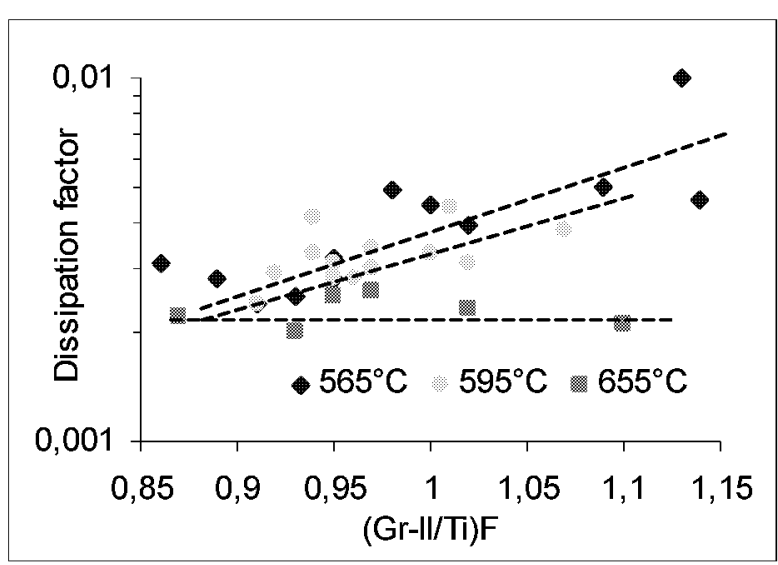

Fig. 6.66: Tan $\delta$ vs. stoichiometry for $30 \mathrm{~nm}$ samples at different growth temperatures. Minimum losses are obtained for the $655^{\circ} \mathrm{C}$ films and become higher with lower $T_{\text {growth }}$.

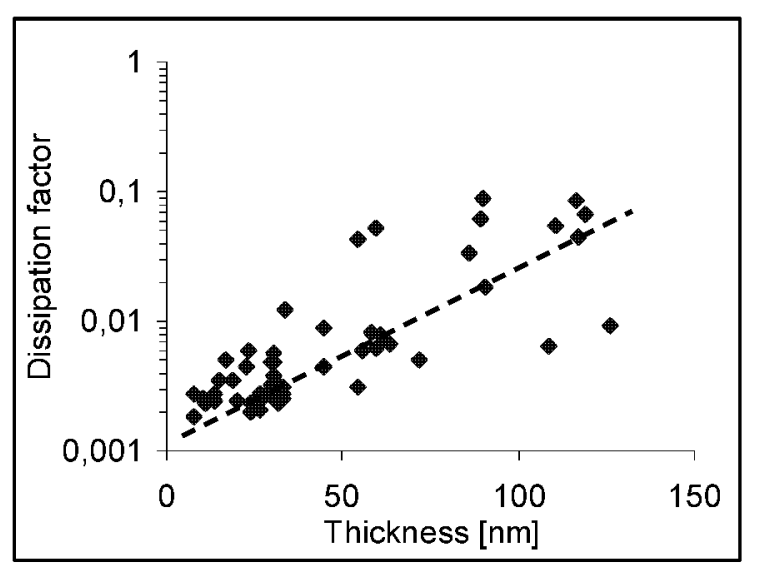

Fig. 6.67: Tan $\delta$ as a function of film thickness at various growth temperatures. There is a clear increase of the losses with increasing thickness that correlates to the higher leakage currents.

Generally, $\tan \delta$ has values between $0.002-0.004$ for the $30 \mathrm{~nm}$ films and shows a slight systematic variation within the investigated stoichiometry range. Nevertheless, an increase of the losses with higher film thickness is observed and thicker samples $\geq 100 \mathrm{~nm}$ display values in the range of 1-2\% (Fig. 6.67). This increase could not be explained with the present structural data as the morphology of thicker samples does not reveal any clues for the increase of the dissipation factor. However, this increase also correlates with the observed higher leakage currents (see below) and the change of the field distribution across the interface layers must be considered in a consistent model of all electrical properties.

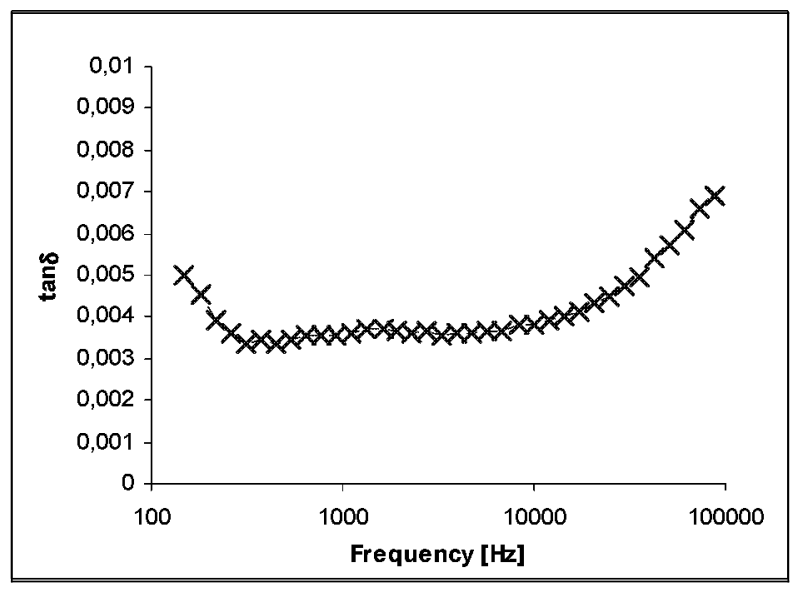

Fig. 6.68 Tan $\delta$ versus frequency plot. The chosen position for the standard electrical characterization was at $1 \mathrm{kHz}$. 
As already shown, the frequency has a minor influence on the permittivity. On the other hand there is a significant impact on the tan $\delta$ value for the used sample geometry and measurement principle. Fig. 6.68 depicts the influence of frequency on the loss tangent for a $30 \mathrm{~nm}$ Ti-rich sample grown at $595^{\circ} \mathrm{C}$. The same qualitative behavior is observed for every sample. There is always a slight increase of $\tan \delta$ at low frequencies, which is attributed to the influence of the leakage current that becomes visible after the decay of the short time relaxation current (see also 6.2.2.3). Relaxation comes into play at higher frequencies and causes the strong increase of the dissipation factor. In the equivalent electrical network, the increase of the losses at the lower frequency regime is attributed to the pole $1 / \omega C_{\mathrm{p}} R_{\mathrm{p}}$ in the relation for the $\tan \delta$ which represents the leakage component (see Eq. 4.22). For the standard electrical characterization we chose a frequency at $1 \mathrm{kHz}$, that combines a sufficient distance from the measurement artifacts with a minimal value for $\tan \delta$.

\subsubsection{Leakage current}

Calculations have shown that the magnitude of the leakage current in case of MOVCD BST thin films should be $<10^{-7} \mathrm{~A} / \mathrm{cm}^{2}$, in order to be used as DRAM capacitors' dielectric. Additionally, a good understanding of the conduction mechanisms in ceramic thin films is essential for their use in integrated devices. A simple way to obtain the leakage current $J_{\mathrm{L}}$ is by applying a voltage step technique, as described in Chapter 4 . The data from the resulting $J_{L}$ vs. $\mathrm{V}$ or $\mathrm{E}$ plots may be fitted into one of the existed models depending on the assumption, whether the charge transport is electrode or bulk limited. In case of an electrode limited conduction many groups suggest a dominating thermionic mechanism that causes emission of electrons from the cathode $[9,27]$. Generally, the examined films appear to be completely depleted (see Section 6.2.1.2). In this case the temperature and field dependence of the leakage current density, $\mathrm{J}_{\text {Schottky, }}$, is given by:

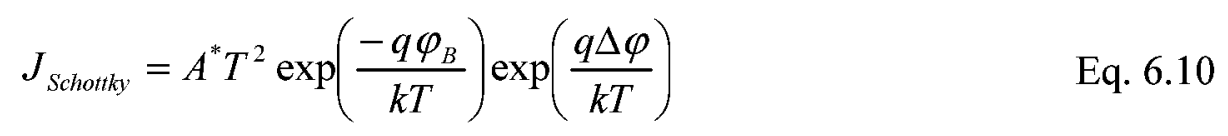

where the barrier lowering $\Delta \varphi$ is defined by:

$$
\Delta \varphi=\sqrt{q E /\left(4 \pi \varepsilon_{r} \varepsilon_{0}\right)}
$$

$\mathrm{A}^{*}$ is the Richardson constant, $T$ the temperature, $\varphi_{\mathrm{B}}$ the barrier height, $\mathrm{k}$ the Boltzmann constant, $q$ the electronic charge, $E$ the applied field, $\mathcal{E}_{\mathrm{r}}$ dielectric permittivity and $\varepsilon_{0}$ the vacuum dielectric constant. Using the appropriate plots, e.g. $\log J$ vs. $\mathrm{E}^{1 / 2}$ the fits yield straight lines and $\varepsilon$ can be calculated from the slope of the fit. Additionally, the barrier height $W_{B}=-q \varphi_{B}$ can be extracted from the interception. Usually, interface layers are inserted in

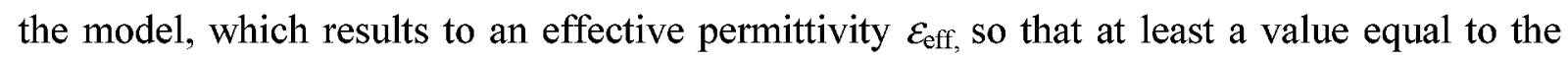
optical permittivity $\varepsilon_{\text {optical }}=5.6$ can be extracted. This value appears reasonable, because the electrons with an energy higher than the barrier height cannot see into the material. 

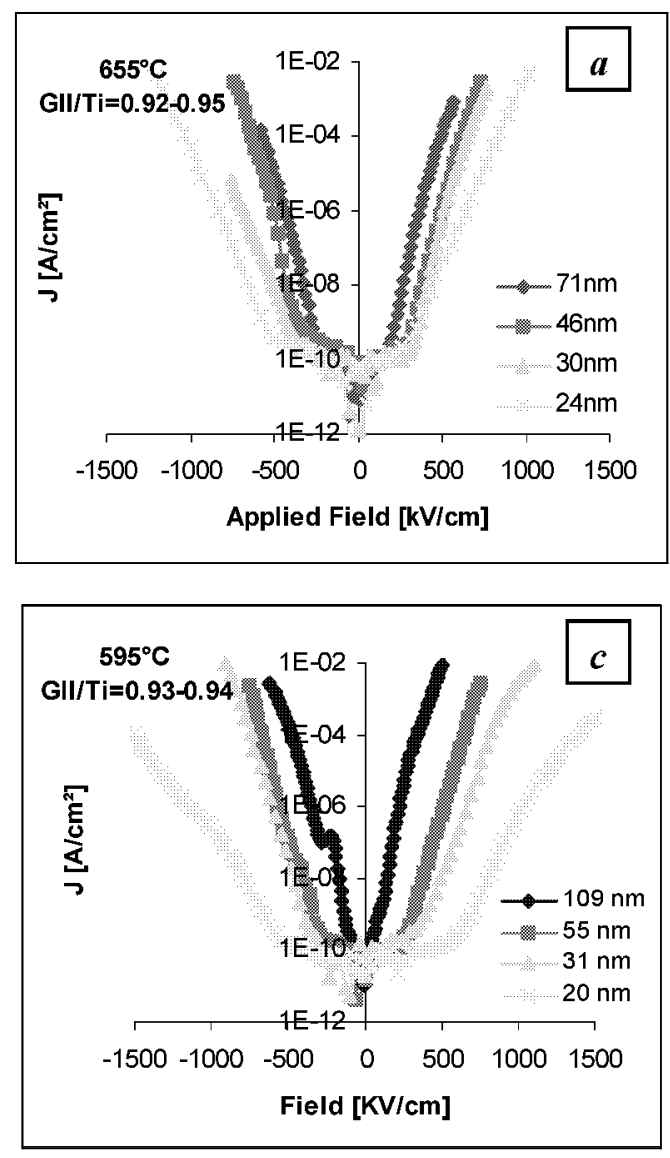
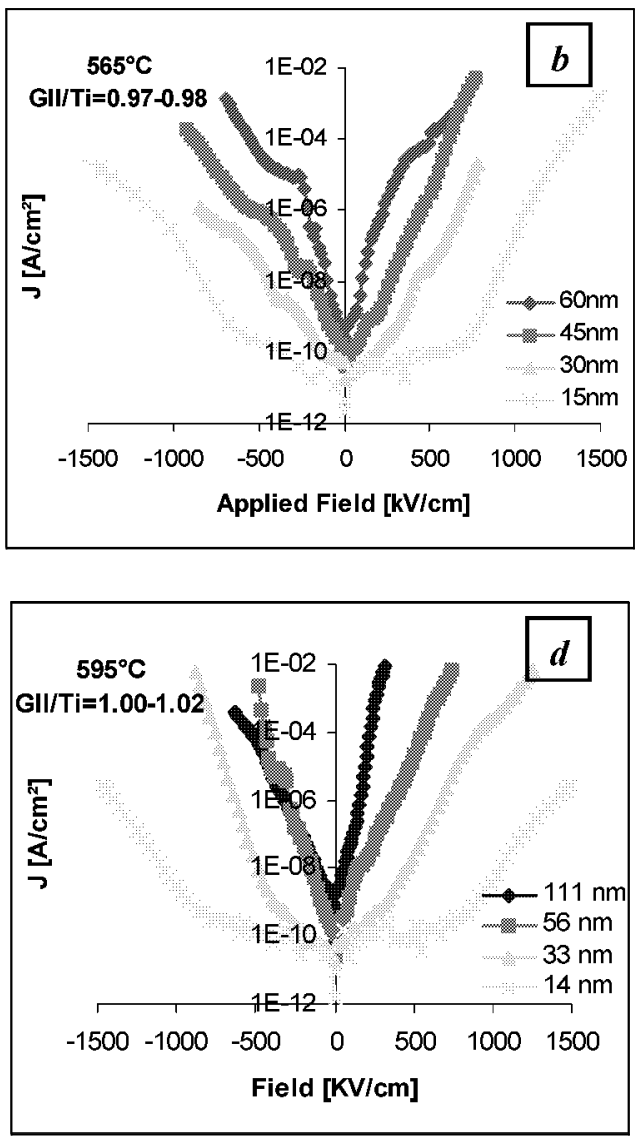

Fig. 6.69: Leakage current for a)-b) samples deposited at different temperatures and c)-d) samples of different stoichiometry at $595^{\circ} \mathrm{C}$.

For the same BST samples with the $\mathrm{C}-\mathrm{V}$ characteristic of Fig. 6.56 we recorded the leakage current as a function of the applied field, since films of different thicknesses have to be compared (Fig. 6.69). All diagrams show a clear dependency of the leakage current on the film thickness, that is not as clearly visible as long as they are plotted versus the bias voltage; the thinner the film, the lower the leakage current for a given applied field. This behavior indicates that the current is not simply controlled by the bulk and details are under active debate [105]. Remarkable are the rather low currents, especially for the 15 and $30 \mathrm{~nm}$ films and the most prominent difference in the field dependence of the currents for films of different thickness. Generally, we obtain a clear influence of the deposition temperature on the magnitude of the leakage current only for small applied fields and films of higher thickness. The leakage current of very thin films $<30 \mathrm{~nm}$ is often expressed through a flat plateau. This shows the resolution limits of our measurement equipment and setup. Measurements with a high precision electrometer in well shielded probe stations achieved a further lowering of the measurement limit of two orders of magnitude, where the leakage current vs. field plots reveal a V-type characteristic. Similar to the C(V) curves, the leakage currents display a strong asymmetry depending on the application of a positive or a negative voltage. This effect is generally attributed to the asymmetrical electrode geometry and processing.

Fig. 6.70 displays the leakage currents values $\left|J_{\text {Leakage }}\right|$ from Fig. 6.69a-d for a constant bias voltage of $1 \mathrm{~V}$, in order to compare the films for an application specific case. Within the measurement precision of our equipment and the reproducibility of our films, high-T 
depositions yield values for the leakage current in the order of $10^{-10} \mathrm{~A} / \mathrm{cm}^{2}$ or below the detection range of our measurement equipment at $1 \mathrm{~V}$ bias. The films obtained at $565{ }^{\circ} \mathrm{C}$ display a thickness dependent leakage. Nevertheless, the magnitude of the leakage current for thin films $<60 \mathrm{~nm}$ is still suitable for DRAM application, thus indicating thin films of high quality even at low deposition temperatures. Investigations on samples of different stoichiometry were restricted on films with a thickness of $\approx 30 \mathrm{~nm}$. Fig. 6.71 summarizes the dependence of the leakage current $J_{\mathrm{L}}$ on the film stoichiometry as a function of the deposition temperature. Unfortunately, these films are grown under non-optimized conditions and we obtain deviations in the magnitude of the leakage current, especially in the low $\mathrm{T}_{\text {growth }}$ regime. Extensive process development led to a decrease of the leakage current to the level shown in Fig. 6.70. Nonetheless, the expected dependence on stoichiometry is suppressed and only a negligible random scattering of the values is observed.

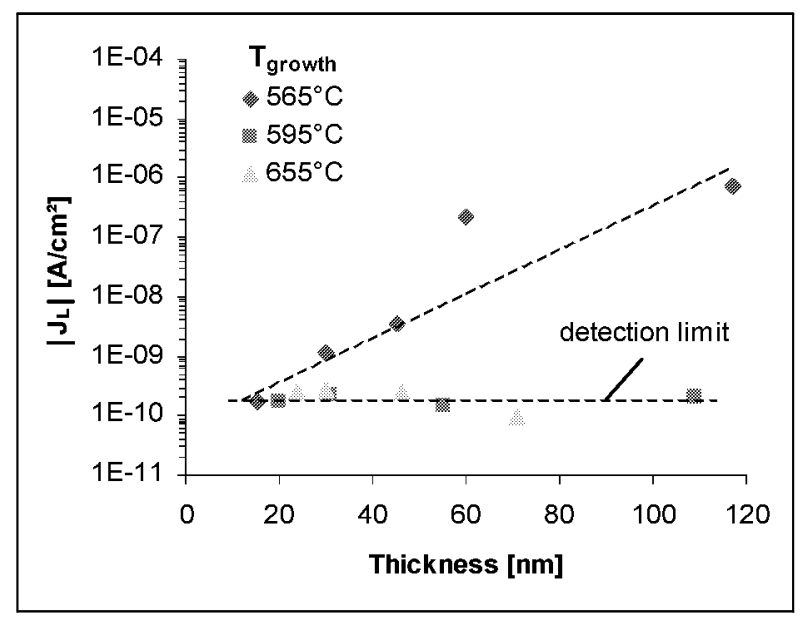

Fig. 6.70: Thickness dependence of the leakage current for an applied $1 \mathrm{~V}$ bias voltage for the samples of Fig. 6.69 .

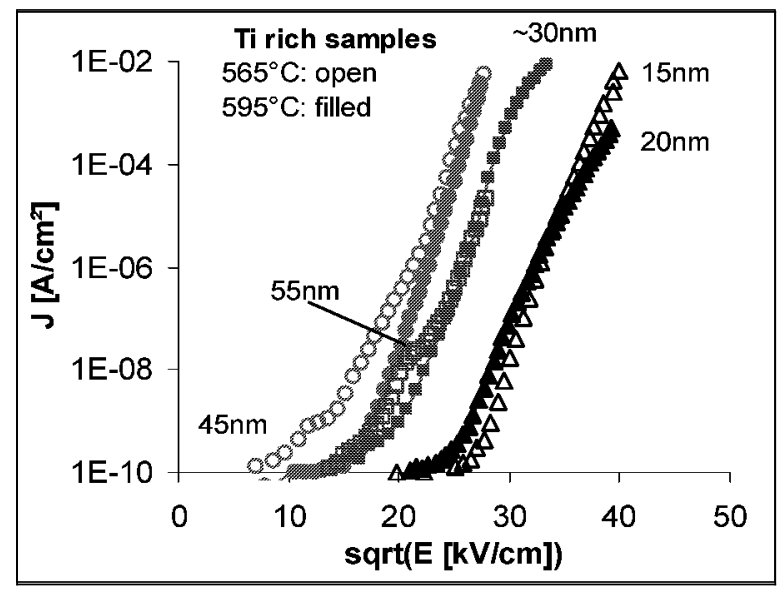

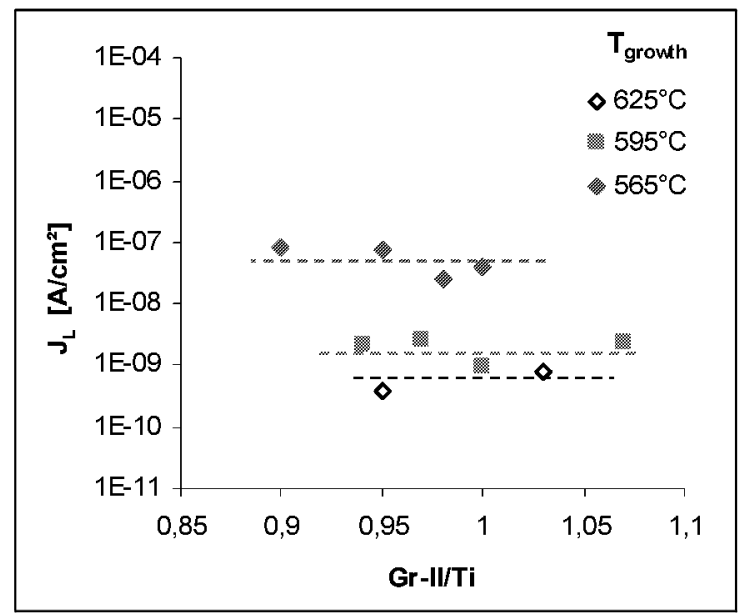

Fig. 6.71: Influence of stoichiometry on the leakage current. Early investigation on $30 \mathrm{~nm}$ samples at $1 \mathrm{~V}$ bias.

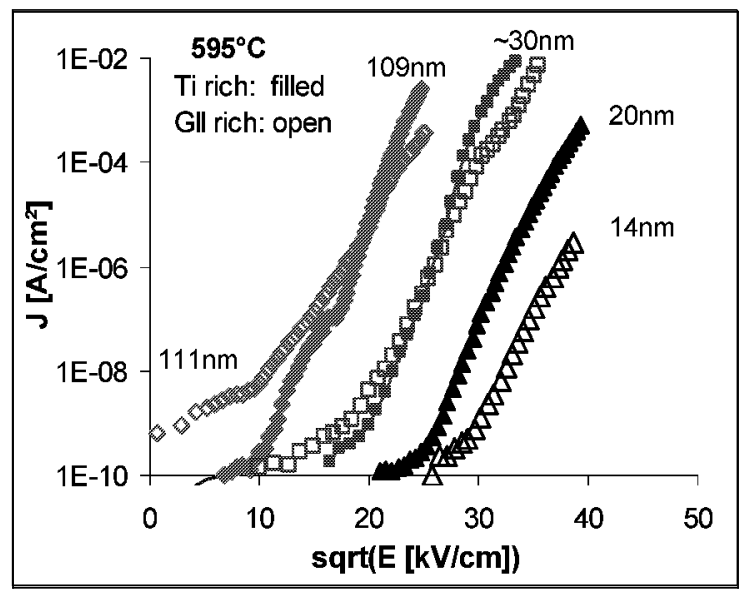

Fig. 6.72: Schottky plots of the field dependence of the leakage current for films of different thickness. a) Ti-rich films (GrII/Ti=0.93-0.98); $T_{d e p}=565^{\circ} \mathrm{C}$ (open symbols) and $595^{\circ} \mathrm{C}$ (closed symbols). b) $T_{\text {dep }}=595^{\circ} \mathrm{C}$; Ti rich (closed symbols) and GrII-rich films (GrII/Ti=1.00-1.02) films (open symbols).

The influence of the deposition temperature and of the stoichiometry is shown in the Schottky plots of Fig. 6.72 which contain data taken from Fig. 6.69. The thickness dependence of the 
leakage current can be understood using the dead layer model which has been previously applied to the permittivity (see Chapter 6.2.1.3). The important point about the understanding of the thickness dependence is the inhomogeneous distribution of the electric field: a large drop within the low $\varepsilon$ interfacial layer and a smaller one within the high- $\varepsilon$ film. Considering the small differences in thickness, Fig. 6.72a shows a small improvement of the leakage with $\mathrm{T}_{\text {dep. }}$. Fig. $6.72 \mathrm{~b}$ shows in addition a smaller slope for the GrII rich films, which might be related to the higher interface capacitance. Details on the modeling of the leakage currents can be found in $[118,119,120]$.

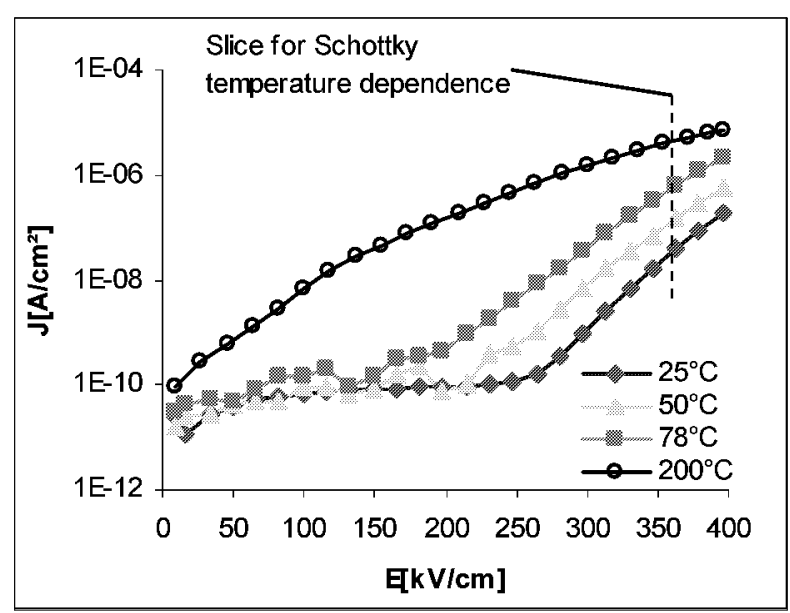

Fig. 6.73: Field dependence of the leakage current for a $60 \mathrm{~nm}$ Ti-rich $595^{\circ} \mathrm{C}$ sample at different temperatures from $25-200^{\circ} \mathrm{C}$.

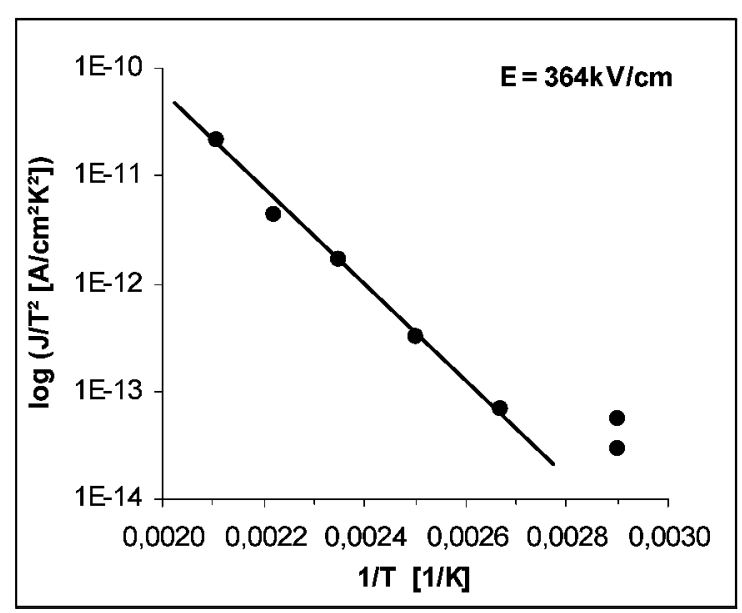

Fig. 6.74: Schottky plot displaying the temperature dependence of the leakage current for an applied field of $364 \mathrm{kV} / \mathrm{cm}$.

Fig. 6.73 shows the influence of temperature on the leakage current behavior of a $55 \mathrm{~nm}$ Tirich sample grown at $595^{\circ} \mathrm{C}$. Generally, the temperature dependence impacts the leakage current strongly. This is presented for a temperature window from room temperature $\left(\sim 25^{\circ}\right)$ to $200^{\circ} \mathrm{C}$. The minimum detection range of the electrometer is clearly visible at low fields as long as the temperature does not exceed $78^{\circ} \mathrm{C}$. With increasing temperature a significant increase of the leakage current is observed for larger electric fields. At $200^{\circ} \mathrm{C}$ a high leakage current flows even at low fields across the capacitor and displays the typical "V" type characteristic. Thus, we obtain an increase of almost two orders of magnitude for the approx. $50^{\circ} \mathrm{C}$ temperature step between room temperature and $78^{\circ} \mathrm{C}$ for an applied field of $275 \mathrm{kV} / \mathrm{cm}$ which corresponds to a voltage of $1.5 \mathrm{~V}$.

Assuming a Schottky emission model one can calculate the effective barrier height from the fit of the temperature dependence in Fig. 6.74. This can be applied to yield the relative permittivity $\varepsilon_{\mathrm{r}}$ and the Richardson constant $\mathrm{A}^{* *}$ from the field dependence. For this, a modified $\log J_{L}-\sqrt{E}$ plot is necessary. For the film discussed here we obtain $\varepsilon_{\mathrm{r}}=0.17$ and $\mathrm{A}^{* *}=3.6 \mathrm{E}-2 \mathrm{~A} / \mathrm{cm}^{2} \mathrm{~K}^{2}$. The resulted permittivity lower than unity and the Richardson constant, which is 4 orders of magnitude lower than the real value of $120 \mathrm{~A} / \mathrm{cm}^{2} \mathrm{~K}^{2}$, cannot be physically explained. The majority of the processed films show similar values. Consequently, the simple thermionic emission model cannot completely explain the obtained leakage current in BST thin films $[9,102,105]$. 


\subsubsection{Relaxation currents}

The relaxation currents control the leakage in the short time region (up to a few seconds) after applying a voltage step. The leakage regime follows the relaxation usually after some tens of seconds and is visible in the flattening of the leakage curves. The relaxation current follows a power law time dependence known as Curie-von Schweidler behavior and drops exponentially by $t^{-\mathrm{n}}$, where $\mathrm{n} \approx 1$ (see Eq. 4.21 ). For the sake of simplicity we assume in the current investigation that $n=1$, even though there are many indications that it might be slightly smaller than unity $(\mathrm{n}<1)$ [106]. The following figures present the polarization currents but the same dependencies are also true for the depolarization currents.

Fig. $6.75 \mathrm{a}$ shows the relaxation current of a $30 \mathrm{~nm}$ sample grown at $595^{\circ} \mathrm{C}$ under two different applied fields. The Curie-von Schweidler behavior is clearly present throughout the time region from $10^{-5} \mathrm{~s}$ to $10 \mathrm{~s}$ up to a field of $500 \mathrm{kV} / \mathrm{cm}$, which is equivalent to approx. $1.5 \mathrm{~V}$. One can see that there is no variation in the magnitude of the relaxation current with increasing voltage. On the other hand, we observe a strong increase of the leakage current, which is now visible within the examined time window after applying a $600 \mathrm{kV} / \mathrm{cm}$ field $(1.8 \mathrm{~V})$. It is also important to say that the leakage current shown in Fig. 6.75a complies with the values measured using the Keithley 617 electrometer along with a Burster 4462 voltage generator, thus proving the consistency of the measurements.
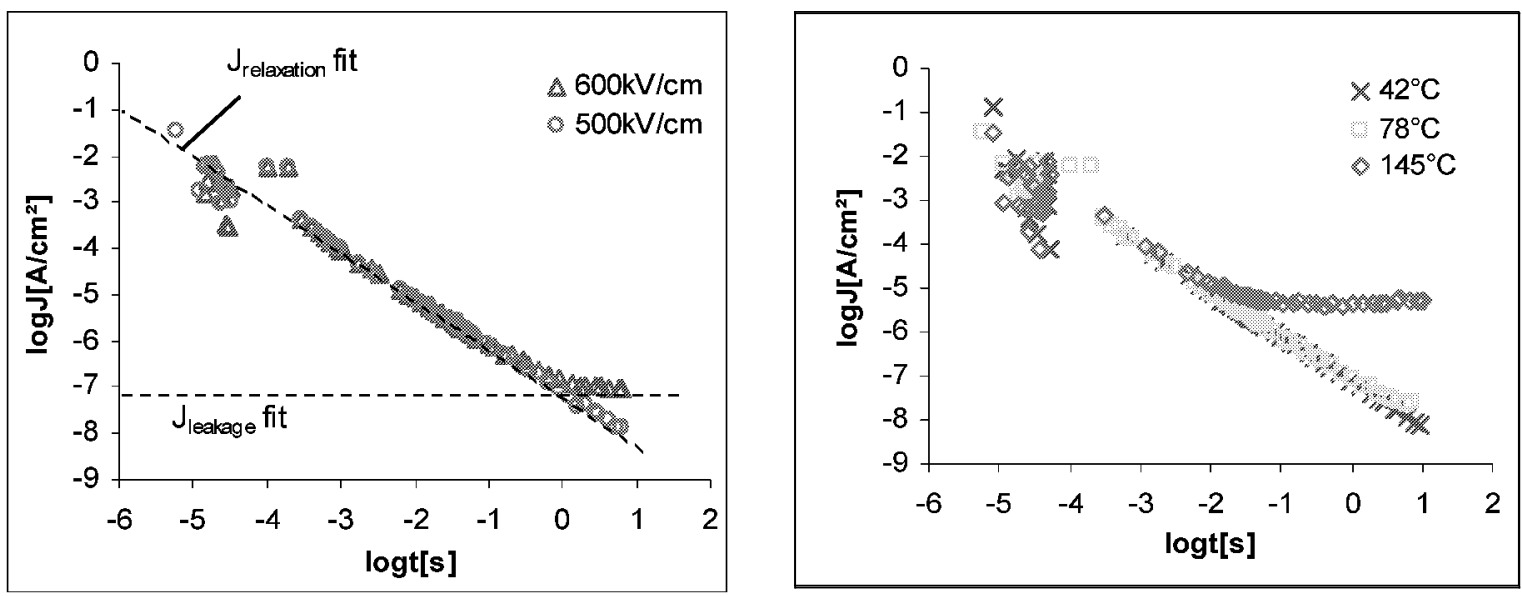

Fig. 6.75: a) Relaxation and leakage current of a $30 \mathrm{~nm}$ stoichiometric sample $\left(T_{\text {growth }}=595^{\circ} \mathrm{C}\right)$ at room temperature b) relaxation properties for the same sample at different temperatures for $a$ constant applied field $(400 \mathrm{kV} / \mathrm{cm})$.

A logarithmic fit is used to calculate the magnitude of the relaxation current. This is demonstrated in Fig. 6.75. The same procedure is necessary in order to determine the level of the leakage current. With the appropriate fit parameters, e.g. magnitude $a$ and slope $n \approx-1$, one can calculate the charge lost during relaxation and leakage phenomena by integrating the $\log (\mathrm{J})-\log (\mathrm{t})$ plots (see Eq. 6.12). The charge loss estimated this way is of the range of 20-30 $\%$ and consequently much higher than the results of DRAM measurements on the same films that are directly proportional to the charge drop, which was no greater than $5 \%$ after one second. Thus, special care must be take not to correlate the constant field relaxation current with the self discharge mechanisms between the refresh times during DRAM operation, since the these relaxation plots provide the charge loss under a constant field (polarization case) or under short-cut condition (depolarization case). This cannot be used to investigate DRAM availability, since it is not describing a simple capacitor's discharge between the refresh 
cycles, where the voltage is just removed after the pulse duration. Hence, the relaxation measurement can only be used to calculate the behavior of DRAM capacitors during charging with very short pulses [9].

The level of the leakage current is considered to be constant with time according to Section 6.2.2.2 and can be directly determined from the plots. Integration of the surface below the $J_{\text {Leakage }}$ fit after Eq. 6.13 yields the charge loss due to the leakage current.

$$
\begin{aligned}
Q_{\text {relaxation }} & =\int_{10^{-9} s}^{1 s} a \cdot t^{-1} d t \\
Q_{\text {leakage }} & =\int_{10^{-9} s}^{1 s} J_{L} d t
\end{aligned}
$$

The temperature dependence of relaxation is presented in Fig. $6.75 \mathrm{~b}$ where the same sample examined in Fig. $6.75 \mathrm{a}$ is measured for a constant field of $400 \mathrm{kV} / \mathrm{cm}$, which is equivalent to a voltage of $1.2 \mathrm{~V}$. The relaxation seems to be independent from temperature, still showing Curie-von Schweidler behavior. Above $78^{\circ} \mathrm{C}$ the leakage current is strongly influenced by temperature as shown in the previous section and at $145^{\circ} \mathrm{C}$ it is approx. three orders of magnitude higher than the relaxation current $10 \mathrm{~s}$ after the voltage step. Measurements at lower temperature are not seriously affected by the leakage current, as the leakage current is still much lower than the relaxation current within the focused time range from $10^{-6} \mathrm{~s}$ to $10 \mathrm{~s}$. The observed independence from temperature is also included in the relaxation mechanism model presented in the Section 4.2.4 as an equivalent network of a capacitor $\mathrm{C}_{\mathrm{HF}}$ and an infinite series of $\mathrm{RC}$ elements in parallel, which is used to explain the $t^{-1}$ behavior. The variation of temperature results in a lowering of the $R_{\mathrm{T}}$ values while $\mathrm{C}$ remains the same. Thus, the time constants $\tau_{\mathrm{T}}=R_{\mathrm{T}} C$ in the infinite series are just rendered and do not influence the current response [102].

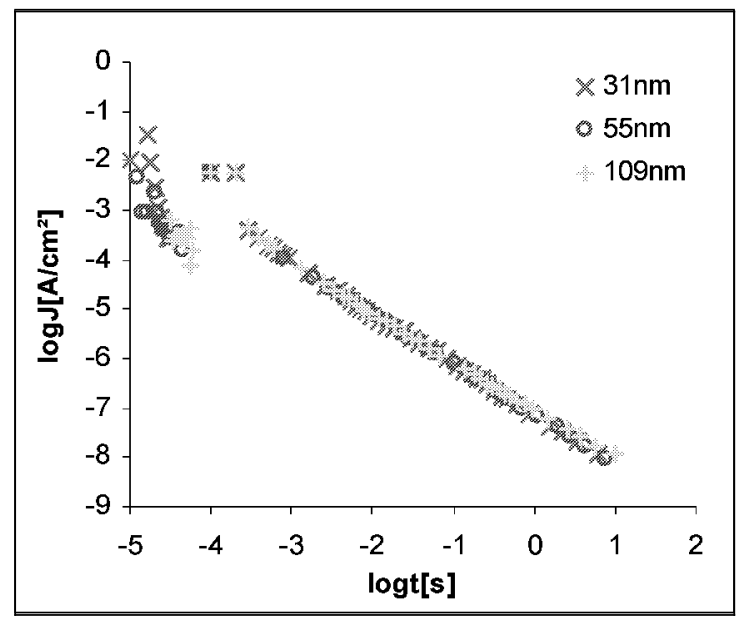

Fig. 6.76: Relaxation currents for BST films with different thickness. All films displayed the same deposition parameters and structure, e.g. Ti-rich composition and $T_{\text {growth }}=595^{\circ} \mathrm{C}$.

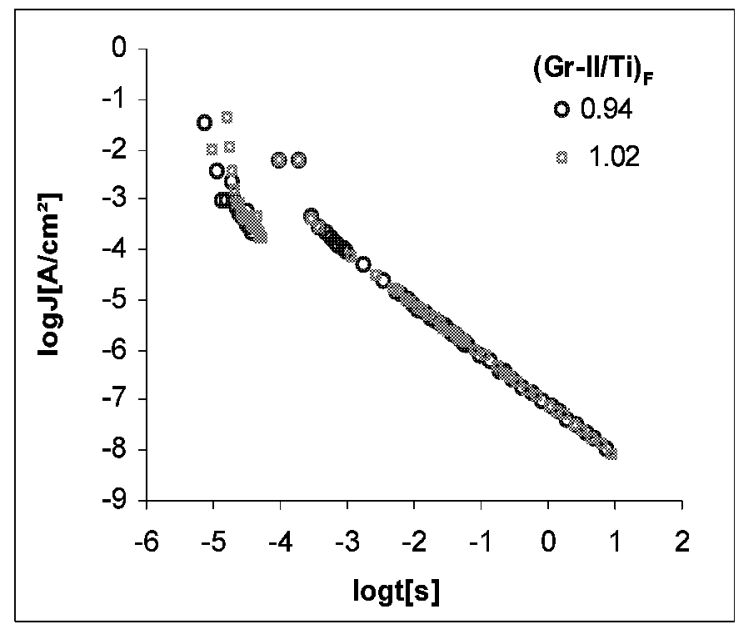

Fig. 6.77: Relaxation currents for films with different stoichiometry. Both films were grown at $595^{\circ} \mathrm{C}$ with a thickness around $30 \mathrm{~nm}$.

Further measurements involved the thickness dependence of the relaxation currents. Fig. 6.76 shows this dependency for three Ti-rich samples grown at $595^{\circ} \mathrm{C}$ with thickness between 
$31 \mathrm{~nm}$ and $109 \mathrm{~nm}$. Examination of films with different thickness showed the same power law time dependence and the same magnitude, which is consistent with the latest measurements from other groups [102], but in contrast with some older publications [106]. Additional investigations included the stoichiometry and growth temperature dependence. Fig. 6.77 shows no stoichiometry influence on the relaxation currents from two $30 \mathrm{~nm}$ films $\left(\mathrm{T}_{\text {growth }}=595^{\circ} \mathrm{C}\right)$. The stoichiometry $(\mathrm{Gr}-\mathrm{II} / \mathrm{Ti})_{\mathrm{F}}$ was varied between 0.94 and 1.02 . Additionally, the influence of the growth temperature could not be determined.

\subsubsection{DRAM pulse measurements}

In order to simulate the DRAM operation we studied the self discharge characteristic of relative large $\left(\sim 8 \approx 10^{3} \mu \mathrm{m}^{2}\right)$ planar BST capacitors. The capacitors were always fully charged with sufficient long pulses. Beyond the leakage through the capacitor, the charge losses must be attributed to the polarization contributions that cannot respond to the quick the read/write times $\left(t_{r / w} \leq 10 n s\right)$ and result in a charge loss. This effect may be negligible in larger capacitors, and it is also important to notice that the values that were measured may be significant different from those of integrated submicron DRAM capacitors. Generally, a 10\% voltage drop is considered a maximum in order for the sense amplifiers to detect the correct logic level.

The determination of the charge loss was performed by a DRAM pulse emulator from aixACCT that was established in our laboratory. Many films manufactured with different deposition parameters undergo routinely the DRAM test procedure, which applies short voltage pulses down to 50ns. The selfdischarge characteristic is recorded in terms of the voltage drop relative to the initial value after a certain time. The typical run of the voltage drop appears to be a linear decay with time. Fig. 6.78 shows a typical run of the DRAM test.

A total of 6 different pulses were applied consecutively $( \pm 200 \mathrm{kV} / \mathrm{cm} \triangleq 0.6 \mathrm{~V} ; \pm 400 \mathrm{kV} / \mathrm{cm} \triangleq$ $1.2 \mathrm{~V} ; \pm 600 \mathrm{kV} / \mathrm{cm} \triangleq 1.8 \mathrm{~V}$ ) and the response of a $30 \mathrm{~nm}$ sample grown at $625^{\circ} \mathrm{C}$ is shown. We observe a $3.9 \%$ relative voltage drop after the application of a $1.2 \mathrm{~V}$ voltage pulse. Additionally, we observe symmetrical behavior after polarization reversal. Extraordinary good DRAM behavior was presented for the $595^{\circ} \mathrm{C}$ sample, e.g. $1 \mathrm{sec}$ after a $700 \mathrm{kV} / \mathrm{cm}(2.3$ V) pulse the voltage drop was $4.2 \%$. An influence of stoichiometry on the charge loss could not be revealed by the DRAM measurements [107].

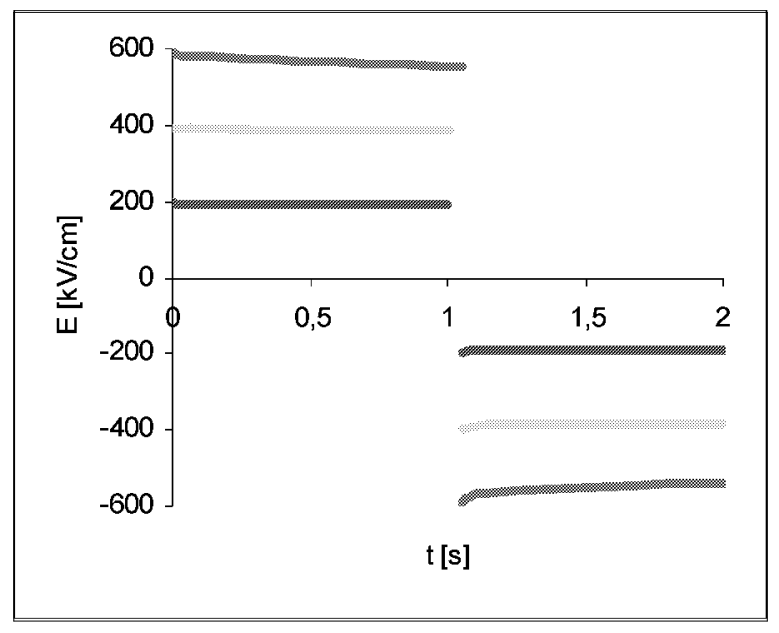

Fig. 6.78: $D R A M$ characteristic of a $30 \mathrm{~nm}$ film grown at $625^{\circ} \mathrm{C} . \mathrm{A} 400 \mathrm{kV} / \mathrm{cm}$ pulse leads to a relative voltage drop of $3.9 \%$ after 1 s.

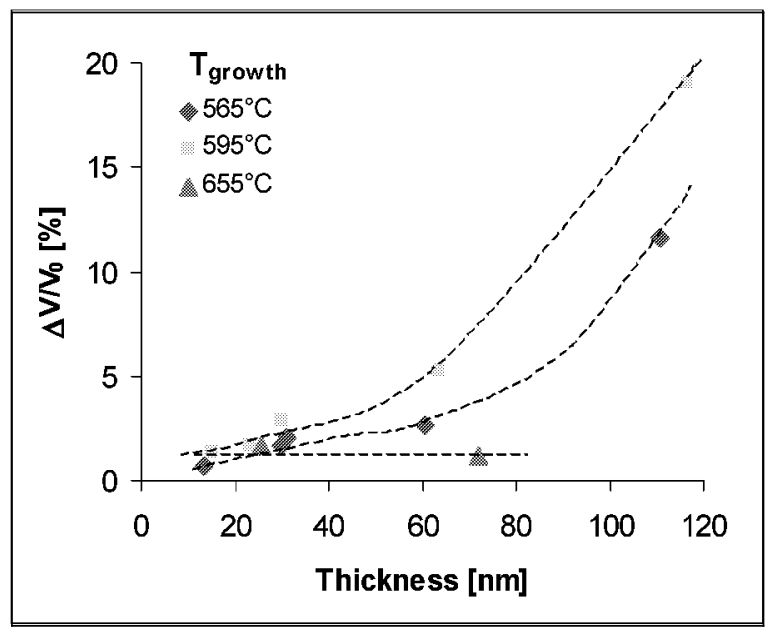

Fig. 6.79: Relative voltage drop $\Delta V / V_{0} \%$ vs. thickness measured at room temperature. 
Since most of the samples display similar DRAM behavior, we chose a somehow different variation in the plotting of the results. Thus, the value of the relative voltage drop $\Delta V / V_{0}[\%]$ one second after the voltage pulse is plotted in the $y$-axis versus a certain film or process parameter.

At first we examined the thickness dependence of the relative voltage drop across the capacitor after a time interval of $1 \mathrm{~s}$ for a given voltage pulse. According to the SIA roadmap for the operation voltage of future DRAMs, this was set to $1 \mathrm{~V}[7,108]$. The time between refresh intervals will be according to the roadmap $1 \mathrm{~s}$ for this technology generation and the self discharge of the capacitor was studied within this time period. The measurements were performed at room temperature in thickness series from $30 \mathrm{~nm}$ to $120 \mathrm{~nm}$ from two different deposition temperatures $\left(565^{\circ} \mathrm{C}, 595^{\circ} \mathrm{C}\right)$. The thickness influence on the DRAM operation is presented in Fig. 6.79.

We generally observe a higher voltage drop with lower deposition temperature for a certain thickness. This difference is rather small as the films thickness remains small and the values are well within the $10 \%$ range. The obtained voltage drop remains quite stable within the measurement precision up to a thickness of $\sim 60 \mathrm{~nm}$. Above this thickness we observe a deviation from the previous behavior and the voltage drop becomes higher exceeding the $10 \%$ limit at the end of a time period of $1 \mathrm{~s}$, which is necessary for the sense amplifier in order to detect a logical state. Generally, we observe a much worse DRAM characteristic for the thicker films $(>100 \mathrm{~nm})$. This is consistent with the degradation of the film properties in this region, that is shown in the previous sections in terms of higher losses, i.e. loss tangent and leakage current values. Concluding, most of the films displayed a relative voltage drop no more than $5 \%$ one second after a single $1 \mathrm{~V}$ pulse. Nevertheless, there are doubts, whether the majority of the results can describe the behavior of submicron storage cells.

The worst case DRAM operation was determined for the case of a first read after a refresh time following a write to the opposite state for a capacitor that was written many times to the same state. The worst case relaxation charge loss averages approximately $10 \%$ for a $30 \mathrm{~nm}$ sample with a capacitance density of $80 \mathrm{fF} / \mu \mathrm{m}^{2}$ [9]. This is 2-3 orders of magnitude higher compared to the leakage current contribution to the voltage drop during the refresh time $\Delta t=1 \mathrm{~s}$, which is:

$$
\Delta V \approx \frac{J \cdot \Delta t}{C / A}
$$

For the previously mentioned sample and a leakage in the range of $10^{-8}-10^{-9} \mathrm{~A} / \mathrm{cm}^{2}$ the voltage drop can be estimated at $0.125 \%-0.0125 \%$. Thus, leakage currents are in most cases too low to produce a voltage drop detectable by our measurements. Consequently, the reason for the voltage drop must be searched in the losses caused by relaxation currents as well as additional error sources induced by the measurement setup, e.g. the leakage through the MOS switch that discharges the capacitor. In a DRAM chip, this is equivalent to the leakage through the access transistor, due to the subthreshold conduction [109]. 


\subsection{Interrelation between film microstructure and electrical properties}

BST thin films of different stoichiometry and thickness were grown at different temperatures. Their microstructural and morphological characteristics were examined in Section 6.1.2 and their electrical properties were presented in Section 6.2. Our investigations provided strong clues that there is a significant relation between the microstructure and the electrical properties the two most important features are summarized in the following:

(i) Deposition Temperature: Major structural changes are observed between deposition temperatures of 565 and $595^{\circ} \mathrm{C}$. At the lowest deposition temperature of $565^{\circ} \mathrm{C}$ polycrystalline growth is observed and for deposition temperatures above $595^{\circ} \mathrm{C}$ we obtain a perfect (100)-fiber structure on Pt-(111), which is revealed by thickness fringes in the XRD scans and very clean and sharp interfaces in HRTEM. Electrical data show an improvement with increasing deposition temperature and excellent electrical properties are obtained for the $655^{\circ} \mathrm{C}$ grown samples. More precisely, we observe a dependence of epsilon on the deposition temperature mainly between $\mathrm{T}_{\text {growth }}=565$ and $595^{\circ} \mathrm{C}$ and these changes could be related to the improved crystalline quality of the films. The lower quality at $565^{\circ} \mathrm{C}$ could also be affected by carbonate formation, as evidenced through FTIR measurements especially for high Gr-II contents. Additionally, an increasing surface roughness, which affects the top electrode must be considered. Furthermore, there is a strong temperature dependence of the bulk permittivity and only a slight variation of the interface capacitance. This different behavior provides a clue for the existence of an interfacial layer, supporting the dead layer theory. In the region of low fields it is always $<10^{-10} \mathrm{~A} / \mathrm{cm}^{2}$ and the leakage current seems less effected by the deposition temperature of the films. However, the differences grow at higher fields.

(ii) Stoichiometry: The incorporation of the excess Gr-II elements yields an increase in the lattice parameter within rather unstable structures which rearrange during post-annealing at $550^{\circ} \mathrm{C}$. Ti excess has no influence on the XRD pattern. There are additional large differences in the grain growth and surface morphology. The correlated changes of the electrical properties are less pronounced. There is a small increase in the effective permittivity with higher Gr-II /Ti ratio; more specifically, there is a strong increase of the interface capacity which overrides the decrease in the bulk value. The leakage current differs again mainly because of the field dependence and this makes comparisons to other related work very problematic.

Finally, the relaxation currents from $10^{-6} \mathrm{~s}$ to $10 \mathrm{~s}$ appear to be independent from the film thickness, stoichiometry, temperature as well as the applied field. Just the leakage current seems to be influenced from the previously mentioned parameters. Ongoing measurements are related to the influence of the growth temperature as well as the deposition method (CVDCSD) on the relaxation currents. The electrical data for the strongly textured films seem well suited for DRAM application, e.g., $\mathrm{C} / \mathrm{A} \approx 60 \mathrm{fF} / \mu \mathrm{m}^{2}$ and $\mathrm{J}=3,7 \cdot 10^{-10} \mathrm{~A} / \mathrm{cm}^{2}$ at $1 \mathrm{~V}$. Even the data for the polycrystalline films, such as $\mathrm{C} / \mathrm{A} \approx 30 \mathrm{fF} / \mu \mathrm{m}^{2}$, are suitable for DRAM application. The determined results comply with the best published data in the high-k dielectrics research and prove our high sample quality and state of the art process using the AIX-2600G MOCVD tool. 


\section{Summary and conclusions}

\subsection{Summary}

This thesis is concerned with the MOCVD growth of BST thin films using a prototype tool for oxide deposition and the systematical understanding of the film properties as a function of their composition and growth parameters.

The scope of this thesis is twofold. From the engineering point of view, an existing MOCVD tool from AIXTRON (2600G3) is optimized for the deposition of ceramic oxides. This reactor is based on the Planetary Reactor ${ }^{\circledR}$ principle and is derived from a successful III-V semiconductor deposition tool. Both reactor and deposition processes are modified to achieve an optimal temperature and process homogeneity. Many changes on the vital components of the system like the liquid delivery system (LDS-300B) from ATMI, the precursor and the gas transfer lines are performed. Extensive process development efforts are applied, in order to turn this prototype MOCVD tool into a production ready, low maintenance tool for industrial application. The optimization process includes a wide range of parameters. Design of experiment methods (DOE) are applied early in many cases to narrow the process window and reduce the huge multidimensional parameter space to a manageable minimum and allow precise statements about the behavior of the reactor.

The scientific part of this thesis considers the systematic investigation and understanding of the properties of the $(\mathrm{Ba}, \mathrm{Sr}) \mathrm{TiO}_{3}$ material system in form of thin films ranging from few nm up to $120 \mathrm{~nm}$. Many of the structural and electrical properties interesting for application are focused and a variety of analyzing techniques are applied. Within the scope of this work, an advanced XRF analytic procedure is developed, in order to achieve a precision $\leq 1 \%$ in thickness and stoichiometry measurements. Our aim is to correlate the obtained results with the deposition parameters, thus being able to control them. The investigations cover all important material aspects of BST in terms of its integration in the existing CMOS process. Finally, a central point in the discussion of the properties is the interrelation between the microstructure of the films and the obtained electrical properties.

The nucleation process is the first important parameter for the understanding of the film microstructure and the nucleation of BST on (111) Pt substrates is studied as a function of the deposition temperature for nominal Ti-rich films. To examine the growth behavior, several ultra-thin films are deposited with an average thickness of $0.5 \mathrm{~nm}, 1.5 \mathrm{~nm}, 5 \mathrm{~nm}$ and beyond. A large number of nucleation spots with a size of $10-20 \mathrm{~nm}$ is visible in the AFM scans after the first deposition step, which coalesce for an average thickness of $1.5 \mathrm{~nm}$ and form a continuous film. In the initial growth stages, an increase of the mean surface roughness is observed with increasing film thickness and, within the precision of the measurements, these trends are independent from the growth temperature.

The desired reduction of the thermal budget during the deposition process, so that the transistors and metalization remain intact, is pursuit. According to the existing roadmaps the growth temperature should not exceed the $500^{\circ} \mathrm{C}$ limitation by far and the deposition temperature has to be further reduced in future DRAM generations [7]. Single step in-situ growth processes to meet this requirement were developed. The lowest possible temperature to grow completely crystalline thin films is found at $565^{\circ} \mathrm{C}$ (susceptor temperature), which corresponds to a $20-50^{\circ} \mathrm{C}$ lower wafer surface temperature depending on the process parameters. The film structure at $565^{\circ} \mathrm{C}$ is polycrystalline and the grain growth is often interrupted. Below this temperature, the crystalline reflexes in the XRD scans are week and the films grow mostly amorphous. Crystallinity becomes better with increasing deposition 
temperature and the films $>595^{\circ} \mathrm{C}$ display excellent (100) orientation on (111) Pt. There is also an indication of an increase of the crystallinity with increasing $\mathrm{Gr}-\mathrm{II}$ content at $595^{\circ} \mathrm{C}$, which is not as prominent for the films grown at $625^{\circ} \mathrm{C}$. The investigation of the material properties of BST at high temperatures displayed excellent structural and electrical properties. On the other hand the low deposition temperature regime is addressed for conformal deposition and a step coverage of $81 \%$ was achieved at $520^{\circ} \mathrm{C}$. Since it is difficult to achieve good electrical properties and sufficient conformality, 2-step processing, low temperature growth and subsequent rapid thermal processing (RTP), has to be considered. The growth of amorphous layers at low temperatures may be combined with the formation of carbonate phases visible in the XRD and FTIR scans. FTIR analysis was used to show the possible elimination of these phases with (RTP).

The stoichiometry, and especially the $(\mathrm{Gr}-\mathrm{II} / \mathrm{Ti})_{\mathrm{F}}$ ratio, has been proven as the most important free parameter for the modification of the film properties. The Ti excess in the films has no impact on the lattice constant, which can be explained by its incorporation at the grain boundaries and the formation of precipitates. In contrast, we observe an increase in the lattice parameter with increasing Gr-II content, that can be explained through its incorporation in form of anti-phase boundaries and Ruddlesden-Popper phases. Additionally, a significant change in the morphology and the resulting topology is visible, depending on the film stoichiometry. Incorporation of the Ti excess generally results into locally more flat films, however, grain growth seems to be not very stable leading to a macroscopically rougher surface due to the formation of large bumps. With increased Gr-II content we obtain very stable columnar grain growth and a locally rougher surface due to the facets of the grains. However, macroscopically, the films are very homogenous. This observation could be understood if the growth of the (100)-oriented crystallites by (111) facets is dominant for GrII rich films, while the Ti-rich films display a less stable (100) growth surface.

The electrical properties are investigated as a function of the previous mentioned parameters. The permittivity increases with increasing deposition temperature, an effect that is attributed to the higher crystallinity grade of the films. At a deposition temperature of $520^{\circ} \mathrm{C}$ we have only partly crystalline films and we find a range of values for the dielectric constant from 38 , for a $31 \mathrm{~nm}$ film with Gr-II excess $(\mathrm{GrII} / \mathrm{Ti}=1.12)$, to 83 for a $59 \mathrm{~nm}$ thick Ti rich $(0.94)$ film. This value of the permittivity corresponds to a specific capacitance of about $12.5 \mathrm{fF} / \mu \mathrm{m}^{2}$. At $565^{\circ} \mathrm{C}$ there is an increase of the specific capacitance to a value around $30 \mathrm{fF} / \mu \mathrm{m}^{2}$. A higher deposition temperature further enhances the permittivity and for $625^{\circ} \mathrm{C}$ we observe a specific capacitance of approx. $60 \mathrm{fF} / \mu^{2}$. Electrical properties also display a dependence on the film stoichiometry. Regarding the permittivity, we observe an increase with growing Gr-II content. Nevertheless, this is visible only at the high $\mathrm{T}_{\text {growth }}$ regime and for the $565^{\circ} \mathrm{C}$ no stoichiometry dependence of the dielectric constant could be determined. The difference in the microstructure for both the low and the high deposition regime and its impact on the electrical properties was further discussed in the light of the dead layer theory. The extracted bulk permittivity and interface capacitance values are similar to existing values from the literature. A lower interface capacitance and a parallel increase of the bulk permittivity for the Ti-rich films is observed. Thus, a layered structure for the enhancement of the effective permittivity, i.e. a thin Gr-II rich interfacial layer followed by a Ti-rich bulk is proposed.

Several loss mechanisms are accounted for within this work and their understanding is essential for the manufacturing of DRAM capacitors. The loss tangent displays values generally between $0.002-0.004$ and a slight increase with growing thickness is revealed. The leakage current is typically in the range of $10^{-9}-10^{-10} \mathrm{~A} / \mathrm{cm}^{2}$ at $1 \mathrm{~V}$ bias for the films grown in the high- $\mathrm{T}_{\text {growth }}$ regime and consequently suitable for DRAM application. In contrast to earlier observations [1] that depict a decrease in the magnitude of the leakage current for a slight Ti- 
rich composition of $\sim 51$ at $\%$ (corresponds to a $(\mathrm{Gr}-\mathrm{II} / \mathrm{Ti})_{\mathrm{F}}$ value of $\sim 0.96$ ), no significant stoichiometry dependence on the leakage currents could be determined, which indicates also that the layer structure proposed above is not corroborated by leakage currents.

Relaxation phenomena after application of a voltage step were studied in detail, since they can be a limiting factor during charging of a storage capacitor. Measurements on fully charged devices depicted no systematic influence of the stoichiometry, thickness and operation temperature on the magnitude of the relaxation currents. However, relaxation measurements are not capable of fully characterizing a capacitor and a DRAM emulation setup was established. The majority of the samples meet the requirements of the industry, i.e. $<10 \%$ voltage drop one second after the application of a $1 \mathrm{~V}$ pulse. Thin films that grow at high temperatures display excellent DRAM characteristics, e.g. a voltage drop of only $2-3 \%$ one second after the application of a $1 \mathrm{~V}$ pulse. Films that grow at $565^{\circ} \mathrm{C}$ still have the potential for DRAM application and a voltage drop no greater than $5 \%$ is obtained for the same conditions.

\subsection{Conclusions and outlook}

A Planetary Reactor ${ }^{\circledR}$, which offers higher throughput and precursor efficiency compared to conventional single-wafer systems, was optimized for oxide deposition. Stable deposition processes were developed in order to produce homogeneous films with excellent electrical properties. Within our research we reproduced the best results reported in the literature. Additionally, stoichiometry was found to be the most important free parameter for the modification of the film properties. Structural investigations showed an influence of the film composition on the surface morphology. A detailed analysis of the electrical data using the "dead layer" model revealed a remarkable change of the model parameters, i.e. bulk permittivity and interface capacitance, with stoichiometry. Thus, we propose a layered capacitor structure for the enhancement of the effective permittivity. Concluding, the MOCVD thin films produced by the AIXTRON 2600G3 tool are already suitable for application in integrated DRAM capacitors.

The discussed properties of the BST films regard planar capacitors, but even if high-k materials are applied $3 \mathrm{D}$ capacitor structures with high aspect ratios are inevitable. Hence, the existing growth processes have to be further developed for conformal deposition and better suited CVD precursors may be necessary. It is possible that the capacitor's dielectric cannot be processed in a single step and a multi-step processing may be favorable. Hence, RTP methods as well as laser annealing technology for crystallization of amorphous ceramic films need to be investigated [111]. In addition to the basic research a second step in the development of the MOCVD tool is necessary in order to qualify the tool for the use in a semiconductor plant. Thus, the deposition processes need to be fully automated and the reliability of the equipment needs to be tested for long term reproducibility. For this, the system needs to be integrated into cluster tools for complete processing including the deposition of appropriate barrier layers and electrodes. 


\section{Literature}

[1] S.R.Summerfelt in "Thin Film Ferroelectric Materials and Devices", R.Ramesh (ed.), Kluwer Acad.Publ., Boston, p.1-42 (1997)

[2] R.E.Jones,Jr., S.B.Desu, "Process Integration for Nonvolatile Ferroelectric Memory Fabrication", MRS Bulletin 21, 6 (1996)

[3] A.Spitzer, „Dielektrische Schichten für die Speicherzelle in DRAMs“ in "Elektrokeramische Materialien - Grundlagen und Anwendungen“, Ferienschule Lectures, Forschungszentrum Jülich GmbH (1995)

[4] E.Adler et. al., „The Evolution of IBM CMOS DRAM Technology”, IBM J. $R \& D, 39$ No.1/2 (1995)

[5] K.Kim, C.-G.Hwang, J.G.Lee, „DRAM Technology Perspective For Gigabit Era”, IEEE transactions on electron devices, 45 No. 3, March (1998)

[6] B.M.Melnick, "Development Challenges of Gigabit Scale 1T-1C DRAMs", presented at the $11^{\text {th }} I S I F$ in Colorado Springs (1999)

[7] Semiconductor Industry Association (SIA) -Roadmap, 2000 update

[8] (C) 2001 Semiconductor Insights Inc., http://www.semiconductor.com/

[9] D.E.Kotecki, J.D.Baniecki, H.Shen et al. ,(Ba,Sr)TiO $\mathrm{T}_{3}$ Dielectrics for Future StackedCapacitor DRAMs", IBM J.Res.Develop. 43-3, 367 (1999)

[10] B.T.Lee,"Integration Processes of $(\mathrm{Ba}, \mathrm{Sr}) \mathrm{TiO}_{3}$ Capacitor for $1 \mathrm{~Gb}$ and Beyond", IEDM proc., 815 (1998)

[11] T.Kawahara et. al., „Preparation of $(\mathrm{Ba}, \mathrm{Sr}) \mathrm{TiO}_{3}$ Thin Films by Chemical Vapor Deposition using Liquid Sources“, J.Appl.Phys. 33, 5897 (1994)

[12] P.Kirlin, S.Bilodeau, P.van Buskirk, "MOCVD of $\mathrm{BaSrTiO}_{3}$ for ULSI DRAMs", Integr.Ferroel. 7, 307 (1995)

[13] A.Beck, J. G.Bednorz, Ch.Gerber, C.Rossel, D.Widmer, „Reproducible Switching in Thin Oxide Ffilms for Memory Applications, Am.Phys.Letters 77, Number 1, 139 (2000)

[14] P.Ehrhart, „Deposition Methods“ in „Neue Materialien für die Informationstechnik“, Ferienschule Lectures, Forschungszentrum Jülich GmbH (2001)

[15] S.Hoffmann, "Modifizierte Erdalkalititanat-Dünnschichten für Integrierte Bauelemente: Morphologie, Dielektrische Eigenschaften und Ladungstransportmechanismen", Ph.D. thesis RWTH Aachen, VDI, Series 9, Nr. 288 (1998)

[16] T.Schneller, IWE RWTH Aachen, private communication (2001)

[17] M.de Keijser, G.J.M.Dormans, "Chemical Vapor Deposition of Electroceramic Thin Films", MRS Bulletin, 21, 6 (1996)

[18] J.F.Scott, F.M.Ross, C.A.Paz de Araujo, M.C.Scott, M.Huffman, " Structure and Device Characteristics of $\mathrm{SrBi}_{2} \mathrm{Ta}_{2} \mathrm{O}_{9}$-Based Nonvolatile Random-Access Memories", MRS Bulletin, 21,7, 33 (1996) 
[19] P. Schäfer, R. Waser, "MOCVD of Perovskite Thin Films Using an Aerosol-Assisted Liquid Delivery System", Adv.Mater.Opt.Elektron. 10, 169 (2000)

[20] M.L.Hitchman, K.F.Jensen, "Chemical Vapor Deposition", Academic Press, London (1993)

[21] L.L.Hench and J.K.West, "Principles of Electronic Ceramics", Wiley \& Sons, NY, 1990

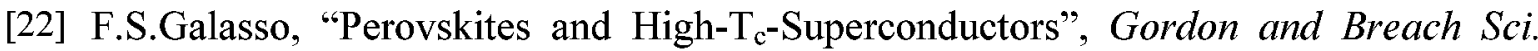
Publ., NY (1990)

[23] G.Arlt, „Werkstoffe der Elektrotechnik“, lecture manuscript, RWTH Aachen (1989)

[24] D.Richter, „Ferroelectrics“ in "Neue Materialien für die Informationstechnik", Ferienschule Lectures, Forschungszentrum Jülich GmbH (2001)

[25] A.D.Hilton, B.W.Ricketts, „Dielectric Properties of $\mathrm{Ba}_{1-\mathrm{x}} \mathrm{Sr}_{\mathrm{x}} \mathrm{TiO}_{3}$ ceramics“, J. Phys. D: Appl. Phys. 29, 1321 (1996)

[26] G.A.Smolenskii, K.I.Rozgechev (1954) taken from Landolt-Börnstein, „Numerical Data and Functional Relationships in Science and Technology: Ferroelectric Oxides", III/16a, New Series, Springer Verlag, Berlin (1981)

[27] G.W.Dietz, "SrTiO Ph.D thesis RWTH Aachen, VDI, Series 21, Nr. 215 (1997)

[28] T.M.Shaw, "The Properties of Ferroelectric Films at Small Dimentions", Annu.Rev.Mater.Sci. 30, 263 (2000)

[29] G.Arlt, D.Hennings, G.de With, "Dielectric properties of fine-grained barium titanate ceramics", J.Appl.Phys. 58, 1619 (1985)

[30] D.L.Polla, L.F.Francis, "Ferroelectric Thin Films in Micro-electromechanical Systems Applications", MRS Bulletin 21, 7 (1996)

[31] R.Waser, S.Hoffmann, "Microstructure-Property Relationships of (Ba,Sr) $\mathrm{TiO}_{3}$ Films", J.Korean Phys.Soc. 32, 1340 (1998)

[32] J.F.Scott et. al., "Dielectric Breakdown in High- $\varepsilon$ Films for ULSI DRAMs: II. BariumStrontium Titanate Ceramics", Integr.Ferroel. 4, 61 (1994)

[33] N.Giannas, "Herstellung strukturierter $\mathrm{SrTiO}_{3}$-Dünnschichten und deren Charkterisierung im Mikrowellen-Frequenzbereich", Diploma thesis RWTH Aachen (2000)

[34] J.D.Baniecki et. al., "Dielectric Relaxation of $\mathrm{Ba}_{0.7} \mathrm{Sr}_{0.3} \mathrm{TiO}_{3}$ Thin Films From $1 \mathrm{mHz}$ to 20GHz", Appl.Phys.Lett. 72 (4), 498 (1998)

[35] C.Basceri, S.K.Streiffer, A.I.Kingon, R.Waser, "The Dielectric Response as a Function of Temperature and Film Thickness of Fiber-Textured (Ba,Sr) $\mathrm{TiO}_{3}$ Thin Films Grown by Chemical Vapor Deposition", J.Appl.Phys. 82 (5), 2497 (1997)

[36] M.E.Lines, A.M.Glass, "Principles and Applications of Ferroelectrics and Related Materials", Harendon, Oxford (1997)

[37] H. Schlichting, "Grenzschicht-Theorie", G. Braun, Karlsruhe (1982) 
[38] G.B. Stringfellow, "Organometallic Vapor-Phase Epitaxy", Academic Press, Boston (1989)

[39] P.Schäfer, „Entwicklung und Aufbau einer Neuartigen MOCVD Anlage zum Wachstum Ferroelectrischer Dünnschichten, Ph.D. thesis RWTH Aachen, Forschungszentrum Jülich (2001)

[40] M.L. Hitchman, "Heterogeneous Kinetics and Mass Transport in Chemical Vapour Deposition Processes", Prog.Crystal Growth Charact. 4, 249 (1981)

[41] M.Dauelsberg „Reaktor und Prozeßoptimierung bei der Metallorganischen GasphasenEpitaxie von III-V-Verbindungshalbleiter, Ph.D. thesis TU Erlangen (1999)

[42] M. Dauelsberg, AIXTRON AG, private communication (2001)

[43] W.S.Rees jr., "CVD of Nonmetals", $V C H$, Weinheim (1996)

[44] C.M.Foster, „Chemical Vapor Deposition of Ferroelectric Thin Films“" in „Thin Film Ferroelectric Materials and Devices", 167, R. Ramesh (ed.), Kluwer Academic Publ. (1997)

[45] F.Felten, J.P.Senateur, F.Weiss, R.Madar, A.Abrutis, „Deposition of Oxide Layers by Computer Controlled Injection-LPCVD", J.Phys. IV France C5, 1079 (1995)

[46] ATMI LDS-300B, ATMI Inc., US Patent \#5204314 (1993)

[47] A.Sherman, "Chemical Vapor Deposition for Microelectronics", Noyes Publications, Ney Jersey (1987)

[48] C.S.Kang, H.J.Cho et. al., "Deposition Characteristics of (Ba, Sr)Ti03 Thin Filrns by Liquid Source Metal-Organic Chemical Vapor Deposition at Low Substrate Temperatures", Jpn.J.Appl.Phys. 36, 6946 (1997)

[49] M.Deschler, E.Woelk, D.Schmitz, G.Strauch, H. Jürgensen, „Multiwafer MOCVD Systems for Ferroelectrics“, Integr.Ferroel. 18, 119 (1997)

[50] P.K.Baumann et. al, „Platinum Electrodes Deposited by Metol Organic Chemical Vapor Deposition for Oxide Application", presented at the $13^{\text {th }}$ ISIF, accepted for publication in Integr. Ferroel. (2001)

[51] J.S.Becker, S.F.Boulyga, „Determination of Stoichiometry and Concentration of Trace Elements in Thin $\mathrm{Ba}_{\mathrm{x}} \mathrm{Sr}_{1-\mathrm{x}} \mathrm{TiO}_{3}$ Perovskite Layers", Fresenius J. Anal. Chem. 370, 527 (2001)

[52] RIGAKU Industrial Corp., ZSX100e Instruction Manual (2001)

[53] R.Tertian, F.Claisse, "Principles of Quantitative X-Ray analysis", Heyden, London (1982)

[54] R.Jenkins, J.L.de Vries, "Practikal X-Ray Spectrometry", Macmillen\&Co.Ltd, London-Basingstoke (1970)

[55] S.Subrahmanyam, E. Goo, "Diffuse Phase Transitions in the $\left(\mathrm{Pb}_{\mathrm{x}} \mathrm{Ba}_{1-\mathrm{x}}\right) \mathrm{TiO}_{3}$ System", J.Mater.Sci. 33, 4085 (1998)

[56] G.Krüger, „Bestimmung der Chemischen Zusammensetzung von BST- und PBTProben mit Hilfe der Sequentiellen RFA", internal report IFF/ZCH, Forschungszentrum Jülich GmbH (2000) 
[57] "Powder Diffraction File - Inorganic Phases", International Center for Diffraction Data (ICDD) (2001)

[58] Philips, X'pert-MRD, operation manual, 950801, $1^{\text {st }}$ ed. (1995)

[59] I.C.Noyan, J.B.Cohen, "Residual Stress, Measurement by Diffraction and Interpretation", Springer NY (1987)

[60] A.Segmüller, M.Murakami, "X-ray Diffraction Analysis of Strain and Stresses in Thin Films" in "Analytical Techniques for Thin Films' (Treatise on Materials Science and Technology", 27, K.N.Tu, R.Rosenberg (eds.), Academic Press (1988) 143- 200

[61] F.Fitsilis et. at. , "BST thin films grown in a multi-wafer MOCVD rector", $J$. Europ.Cer.Sos. 21, 1547 (2001)

[62] P.Williams, "Quantitative Analysis Using Sputtering Techniques: Secondary Ion and Sputtered Neutral Mass Spectrometry", in "Practical Surface Analysis (2nd Ed) Vol 2: Ion and Neutral Spectroscopy", D.Briggs, M.P.Seah (eds.), J. Wiley \& Sons, New York, 177-228 (1992)

[63] Günzler, Böck, "IR-Spektroskopie, eine Einführung”, VCH(1983)

[64] "TF analyzer 2000", DR/RX-Module Operation Manual, aixACCT (2001)

[65] "HP4284A Precision LCR Meter Operation Manual", HP Part No.04284-90020, Hewlett Packard (1996)

[66] W.L.Warren, D.Dimos, R.M.Waser, „Degradation Mechanisms in Ferroelectric and High-Permittivity Perovskites, MRS Bulletin 21, 7 (1996)

[67] S.Zafar et. al., „Resistance Degradation in Barium Strondium Titanate Thin Films“, J.Appl.Phys. 86 (7), 3890 (1999)

[68] „Bedienungsanleitung DIGISTAND Burster 4462“, Burster (2000)

[69] „Model 6517A Electrometer User’s Manual“, Keithley Instruments, Doc. Num. 6517A-900-01 Rev. A (1996)

[70] S. Schmitz, IFF/FZJ, private communication (2001)

[71] S.Strapatsakis, "Modification and Optimization of an Automatic Probe Station for the Electrical Characterization of Electroceramic Thin Films", Diploma thesis $R W T H$ Aachen (2001)

[72] J.G.Simmons ,Theory of Metallic Contacts on High Resistivity Solids-I. Shallow Traps", J.Phys.Chem.Solids 32, 1987 (1970)

[73] J.G.Simmons „Theory of Metallic Contacts on High Resistivity Solids-II. Deep Traps", J.Phys.Chem.Solids 32, 2581 (1971)

[74] C.S.Kang, H.-J.Cho, C.S.Hwang, B.T.Lee, K.-H.Lee, H.Horii, W.D.Kim, , S.I.Lee, a M.Y.Lee, "Deposition characteristics of $(\mathrm{Ba}, \mathrm{Sr}) \mathrm{TiO}_{3}$ thin films by liquid delivery MOCVD at low temperatures", Jpn.J.Appl.Phys. 36, 6946 (1997)

[75] H.Shen, D.E.Kotecki, R.J.Murphy, M.Zaitz, R.B.Laibowitz, T.M.Shaw, K.L. Saenger, J.Banecki, G.Beitel, V.Klueppel, H.Cerva, MRS Symp.Proc. 493, 33 (1998) 
[76] T.Horikawa, M.Tarutani, T.Kawahara, M.Yamamuka, N.Hirano, T.Sato, S. Matsuno, T.Shibano, F. Uchikawa, K.Ono, T.Oomori, MRS Symp.Proc. 541, 3 (1999)

[77] Laboratoires d'Electronique Philips, European Patent EP 0334433 B1 (1993)

[78] Advanced Technology Materials, Inc., „Operating Manual LDS-300B“, Version 5.2

[79] P.C.Van Buskirk, J.F.Roeder, S.Bildeau „Manufacturing of Pervoskite Thin Films using Liquid Delivery MOCVD", Integr. Ferroel. 10, 9 (1995)

[80] T.Kawahara, M.Yamamuka, A.Yuuki, K.Ono, „,(Ba,Sr)TiO ${ }_{3}$ Films Prepared by Liquid Source Chemical Vapor Deposition on Ru Electrodes“, Jpn.J.Appl.Phys. 354880 (1996)

[81] Cooperation AIXTRON-IFF/EKM, $1^{\text {st }}$ internal report 1HY (1999)

[82] Cooperation AIXTRON-IFF/EKM, $2^{\text {nd }}$ internal report $2 \mathrm{HY}$ (1999)

[83] Cooperation AIXTRON-IFF/EKM, $3^{\text {rd }}$ internal report $1 \mathrm{HY}(2000)$

[84] Cooperation AIXTRON-IFF/EKM, $4^{\text {th }}$ internal report $2 \mathrm{HY}(2000)$

[85] Cooperation AIXTRON-IFF/EKM, $5^{\text {th }}$ internal report $1 \mathrm{HY}(2001)$

[86] F.Fitsilis, S.Regnery, P.Ehrhart, R.Waser, F.Schienle, M.Schumacher, H.Juergensen, "Structure Property Relations of BST Thin Films", Integr.Ferroel. 38, 211 (2001)

[87] P.Ehrhart, F.Fitsilis, S.Regnery, R.Waser, F.Schiente, M.Schumacher, M. Dauelsberg, P.Strzyzewski, H.Jürgensen, "Deposition of Thin BST Films in a Multi-Wafer Planetary Reactor", Integr.Ferroel. 30,183 (2000)

[88] D.C.Kim, W.Jo, H.M.Lee, K.Y.Kim „Properties of $(\mathrm{Ba}, \mathrm{Sr}) \mathrm{TiO}_{3}$ Thin Films Prepared by Liquid Source Delivery Chemical Vapor Deposition", Integr.Ferroel. 18, 137 (1997)

[89] M.Schumacher, J.Lindner, P.Strzyzewski, M.Dauelsberg, H.Juergensen, „MOCVD Processed Ceramic Thin Film Layers for Future Memory Applications", Semicond. Fabtech $11^{\text {th }}$ ed., 227 (2000)

[90] C.L.Jia, IFF/FZJ, private communication (2002)

[91] E.H.Nicollian, J.R.Brews, "MOS Physics and Technology", John Wiley\&Sons (1982)

[92] H.Yabuta et.al, "Improved Electrical Properties of $\mathrm{SrTiO}_{3}$ Thin Films by Electron Cyclotron Resonance Plasma Chemical Vapor Deposition", Mat.Res.Soc.Symp.Proc. 361, 325 (1995)

[93] P.C.Van Buskirk, S.Bilodeau, J.F.Roeder, P.S.Kirlin, “ Metalorganic chemical vapor deposition of complex metal oxide thin films by liquid source chemical vapor deposition", Jpn.J.Appl.Phys. 35, 2520 (1996)

[94] R.A.Nyquist, R.O.Kagel, "IR spectra of inorganic compounds", Academic Press, NY and London (1971)

[95] A.I.Kingon, S.B.Desu, „Meterials Research Society Tutorial: Ferroelectric Thin Films“, MRS Spring Meeting (1996)

[96] X.Chen, A.I.Kingon, H.N.Al-Shareff, K.R.Bellur, K.Gifford, O.Auciello, "Leakage and Interferenz Engineering in Titanate Thin Films for Non-Volatile Ferroelectric Memory and ULSI DRAMs", Integr. Ferroel. 7, 291 (1995) 
[97] J.Park, C.S.Hwang, D.Y.Yang, "Optimization of the annealing process for the (Ba,Sr)Ti $\mathrm{O} 3$ thin films grown by low-temperature $\left(420^{\circ} \mathrm{C}\right)$ metalorganic chemical vapor deposition", J.Mater.Res. 16 No.5 (2001)

[98] M.Yoshida, H.Yabuta, S.Sone, H.Yamaguchi, K.Arita, and Y.Kato, "Phase Formation in CVD-Grown $\mathrm{StTiO}_{3}$ and $(\mathrm{Ba}, \mathrm{Sr}) \mathrm{TiO}_{3}$ Films", Electrochem.Soc.Proc. 97-25, 1109 (1997)

[99] S.Stemmer, S.K.Streiffer, N.D. Browning, and A.I.Kingon, "Accommodation of Nonstoichiometry in (100) Fiber-Textured $\left(\mathrm{Ba}_{\mathrm{x}} \mathrm{Sr}_{1-\mathrm{x}}\right) \mathrm{Ti}_{1+\mathrm{y}} \mathrm{O}_{3+\mathrm{z}}$ Thin Films Grown by Chemical Vapor Deposition“, Appl.Phys.Letters 74, 2432 (1999)

[100] J.C.Shin, J.Park, C.S.Hwang, H.J.Kim, „Dielectric and electrical properties of sputter grown (Ba,Sr) $\mathrm{TiO}_{3}$ thin films", J. Appl. Phys. 86, 506 (1999)

[101] A.I.Kingon, S.K.Streiffer, C.Basceri, S.R.Summerfelt, "High-permittivity perovskite thin films for dynamic random-access memories", MRS Bulletin 21, 7, 46 (1996)

[102] G.Steinlesberger, H.Reisinger, H.Bachhofer, H.Schroeder, W.Werner „Dielectric Relaxation and Charge Transport Mechanism in $(\mathrm{Ba}, \mathrm{Sr}) \mathrm{TiO}_{3}$ Thin Films“, accepted for publication in Integr.Ferroel. (2001)

[103] G.Steinlesberger, "Untersuchung der elektrischen Eigenschaften von Dünnschicht Barium-Strontiumtitanat", Diploma thesis TU Wien (2000)

[104] S.K.Streiffer, C.Basceri, C.B.Parker, S.E.Lash, A.I.Kingon, "Ferroelectricity in thin films: The dielectric response of fiber-textured $\left(\mathrm{Ba}_{\mathrm{x}} \mathrm{Sr}_{1-\mathrm{x}}\right) \mathrm{Ti}_{1+\mathrm{y}} \mathrm{O}_{3+\mathrm{z}}$ thin films grown by chemical vapor deposition", J.Appl.Phys. 86, 4565 (1999)

[105] S.Schmitz, H.Schroeder „Electrical Characterization of $\mathrm{SrTiO}_{3}$ Thin Films“, Poster presentation at the $13^{\text {th }} \operatorname{ISIF}(2001)$

[106] M.Schumacher, "Relaxationsphänomene in keramischen Dünnschichten für zukünftige hochintegrierte Halbleiterspeicher", Ph.D thesis RWTH Aachen (1998)

[107] P.Ehrhart, F.Fitsilis, S.Regnery, C.L.Jia, H.Z.Jin, R.Waser, F.Schienle, M. Schumacher, H.Juergensen, "Growth of $(\mathrm{Ba}, \mathrm{Sr}) \mathrm{TiO} 3$ Thin Films in a Multi-Wafer MOCVD Reactor”, Mat.Res.Soc.Symp.Proc. 655, CC9.4.1. (2001)

[108] Semiconductor Industry Association (SIA), Roadmap, 2001 Edition

[109] H.B.Bakoglu, "Circuits, Interconnections, and Packaging for VLSI", Addison Wesley (1990)

[110] R.Chau et. al., "A 50nm Depleted-Substrate CMOS Transistor (DST)", IEDM Proc. (2001)

[111] O.Baldus, R. Waser, E.W. Kreutz, „Laserannealing Studies of Barium Strontium Titanate Thin Films,, Integr.Ferroel. 30, 129(2000)

[112] C. Zhou, D.M. Newns, "Intrinsic Dead Layer Effect and the Performance of Ferroelectric Thin Film Capacitors", J.Appl. Phys. 82, 3081 (1997)

[113] C.T. Black, J.J.Welser, "Electric Field Penetration into Metals: Consequences for High-Dielectric-Constant Capacitors", IEEE Trans.Electron.Dev. 46, 776 (1999)

[114] P.Schäfer, P.Ehrhart, F.Fitsilis, W.Ma, S.Regnery, "Deposition of Electroceramic Thin Films by MOCVD", Internal report, IFF Forschungszentrum Jülich GmbH (1999) 
[115] F.Fitsilis, S.Regnery, P.Ehrhart, "Microstructural and Electrical Properties of Electroceramic Thin Films Deposited by MOCVD", Internal report, IFF Forschungszentrum Jülich GmbH (2000)

[116] R.Jenkins, “An Introduction to X-Ray Spectrometry”, Heyden, New York (1974)

[117] P.Hahn-Weinheimer, A.Hirner, K.W.Diefenbach, "Röntgenfluoreszenz-analytische Methoden", Vieweg, Braunschweig (1995)

[118] H.Schroeder, S.Schmitz, P.Meuffels, "Simulation of leakage current in thin films with dead layers", presented at the IFFF 2002 in Nara, accepted for publication in Integr. Ferroel. (2002)

[119] S.Schmitz, H.Schroeder, "Leakage current measurements of STO and BST thin films interpreted by the "dead" layer model", presented at the IFFF 2002 in Nara, accepted for publication in Integr.Ferroel. (2002)

[120] S.Schmitz, "Abhängigkeit des Leckstromes und der Dielektrizitätskonstanten in $\mathrm{SrTiO}_{3}$ - und (Ba,Sr) TiO ${ }_{3}$-Dünnschichtkondensatoren von der Kontaktmetallisierung", Ph.D. thesis RWTH Aachen, Forschungszentrum Jülich (2002) 


\section{Acknowledgments}

This dissertation arose during my work at the Institut für Festkörperforschung (Institute for Solid State Research) at the Forschungszentrum Jülich (Jülich Reseach Center) in the years from 1999 to 2002. I would like to thank all my colleagues in Jülich as well as those at the Institute for Werkstoffe der Elektrotechnik (Institut for Materials in Electrical Engineering) at the University of Aachen for their valuable support.

I would like to express my deep appreciation to Prof. R. Waser for providing excellent conditions to carry out my research on such an exciting field.

I would like to thank Prof. M. Heuken for his interest in my topic and for being the coexaminer of my work.

Especially, I would like to thank my supervisor Dr. Ehrhart for his support and the many fruitful and stimulating discussions which have finally led to this publication.

During the development of the XRF analytical methods, I profited from the extensive knowhow of W. Krumpen. Additionally, I would like to mention the valuable help of S. Regnery, in the MOCVD growth of the films. My roommates, S. Schmitz an P. Schäfer deserve my appreciation for supporting me during the long road to my Ph.D.

I have to thank the colleagues who contributed to my work: Dr. C. Szot and Dr. C. Jia for the AFM and the HRTEM scans, respectively; R. Gerhart and D. Vukelic for their help in the fabrication of the capacitors and post-processing; G. Wasse for the SEM pictures and Dr. U. Breuer for the SIMS/SNMS analysis.

Further, I would like to thank the managers, engineers and technicians from AIXTRON AG, which helped us to bring this project to completion.

Last, but not least, I must acknowledge the contribution of the many students who worked for me during their diploma or student thesis: N. Giannas, S. Strapatsakis, C. Christidis, G. Nanos, K. Eftimiadis and P. Kalaitzidis. 



\section{Forschungszentrum Jülich

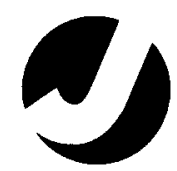

Jül-4001

August 2002

ISSN 0944-2952 